\title{
Stories Teachers Tell: A Narrative Exploration of American Sign Language Teachers' Professional Lives
}

\author{
by
}

Christina Doré

A thesis submitted to the Faculty of Graduate and Postdoctoral Affairs in partial fulfillment of the requirements for the degree of

Doctor of Philosophy

in

Applied Linguistics and Discourse Studies

Carleton University

Ottawa, Ontario

(C) 2020 Christina Doré 


\begin{abstract}
American Sign Language (ASL) is one of the most popular languages for foreign language study among hearing adult learners. ASL teaching has deep connections to the long-standing oppression of deaf people and sign languages, and so ASL teachers might be linguistic and cultural guides as well as deaf advocates, allies, and spokespeople. Yet, little research has addressed who ASL teachers are and how they have come to and navigated the profession. In Canada, there is no clear educational pathway to learning to teach ASL. Although formal and informal teacher learning opportunities exist, they are not widely adopted or enforced.
\end{abstract}

In response, this narrative dissertation explored the professional life histories, or pathways, of seven ASL teachers in Canada through multi-part interviews. I was informed by theories of narrative as a social practice and teacher learning as embodied, and prior literature about the sociohistorical context of ASL and ASL teaching in North America. To further contextualize teachers' stories, I also conducted interviews with representatives from deaf cultural organizations and ASL program administrators and incorporated publicly available data about ASL in Canada.

The findings of this study were an extensive collection of stories drawn from the seven teachers' accounts, organized into three chronological clusters: early ASL and teaching experiences (pathways to teaching), ongoing teaching experiences (pathways through teaching), and reflections on experience (pathways forward). Stories about teachers' early experiences underscored the diversity of people that comprised this workforce - native 
and non-native signers, deaf, hearing, and hard-of-hearing, formally and informally trained, and so on. Their accounts of ongoing practice illustrated how they variously strove to be teachers and the different successes and challenges they met along the way in meeting their goals. Teachers' closing reflections demonstrated that their teaching work was tightly intertwined with other goals, including the broader social justice aim of improving the status of sign languages and deaf people in Canada.

This study aimed to make a space for ASL teachers in academic conversations where they are rarely featured. I hoped that ASL teachers, especially the study's participants, found meaningfulness in reflecting and sharing professional experiences documented in this dissertation. 


\section{Acknowledgements}

My wonderful ASL teacher-participants, thank you for sharing your stories so warmly and so generously with me. This dissertation is for you.

Dr. Janna Fox, my supervisor, thank you for everything. Without you this "beast" would never (ever) have finished — or started, for that matter! Thank you for your infinite patience and wisdom over these many years, and for being the loudest cheerleader in my sprint to the finish line.

Dr. Natasha Artemeva, thank you for your calm and nurturing guidance always, from my first campus visit in 2011 to the end of this Ph.D. nearly ten years later. Thank you for all of the great theorists you brought to life.

Dr. Samah Sabra, a.k.a. "who I want to be when I grow up", thank you for showing me how to be a caring scholar and educational developer.

Dr. Peggy Hartwick, thank you for your brilliant commentary on this final document, and for everything in the years leading up to now-the writing sessions, the snack-chats, the listening, and all of the support. 
Dr. Sophie Tamas, thank you for coming into my research journey at just the right time, when I was feeling most unsure and in need of alternate perspectives on life and research, and for being there to see me out the other side.

Dr. Antoinette Gagné, thank you for being a generous, insightful, and thorough reader of my work, and for your many ideas about how I can get this work "out there".

The dream team of Joan Grant, Connie Wall, and Tracey Wright, thank you for answering a thousand questions, booking a thousand rooms, sharing staplers and sweets and so many kind words of encouragement.

The incredible group of intelligent, fun, and funky women who kept me company during a billion writing units, thank you for the inspiration, the laughter, and the 45-minute silences, and for sharing your brains and your hearts during this long journey.

Janna Klostermann, thank you for being your inimitable self and for the extra walk-andtalks about narrative and research and \#PhDlife, especially in the last few months.

Josée-Anna Tanner, friend, colleague, co-editor, co-researcher, teacher extraordinaire, etc. etc. etc., thank you for keeping me company and helping me back up when the road got too bumpy to stand. 
My crew of proofreaders, thank you for rallying at the drop of a hat to smooth out all of the wrinkles in this mountain of pages.

My friends and family near and far, thank you for balancing genuine curiosity about my research and genuine understanding when I. Did. Not. Want. To. Talk. About. It.

My family—my parents, grandparents, sisters, and in-laws, thank you for sharing the struggle and the celebration with me, year after year.

My husband, Liam—what a life! Thank you for letting me have my cake and eat it, too. 


\section{Table of Contents}

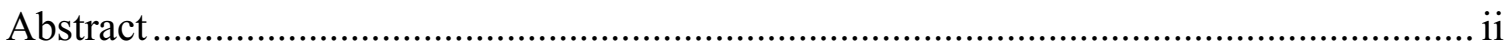

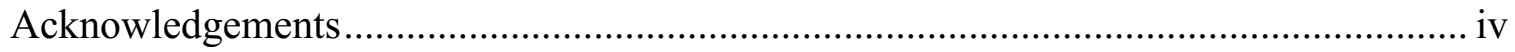

Table of Contents ................................................................................................. vii

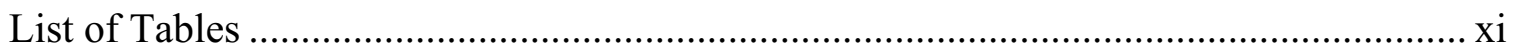

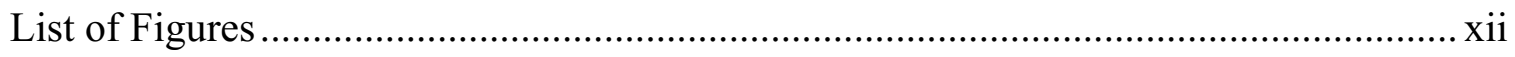

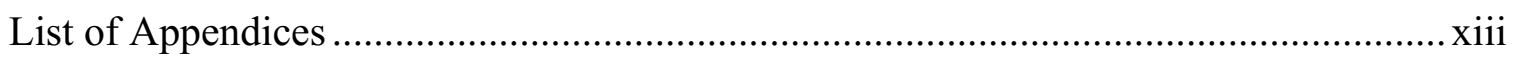

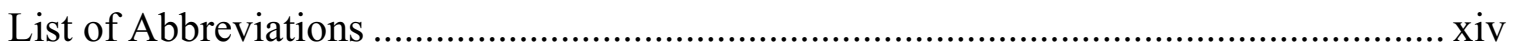

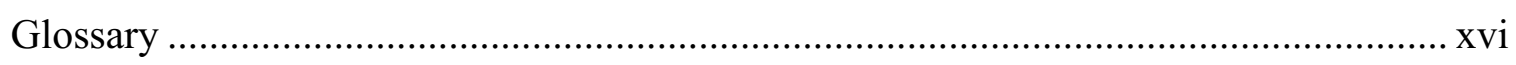

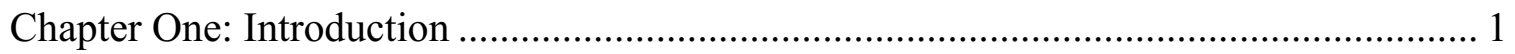

Self-Introduction .................................................................................. 4

Guiding Research Questions ................................................................... 5

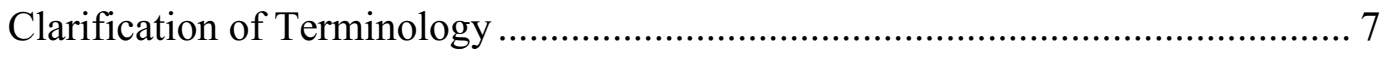

Theoretical Framework ............................................................................. 16

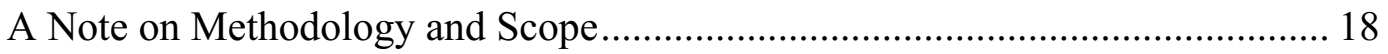

Intended Contributions.......................................................................... 20

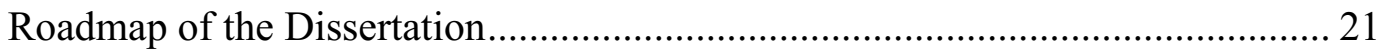

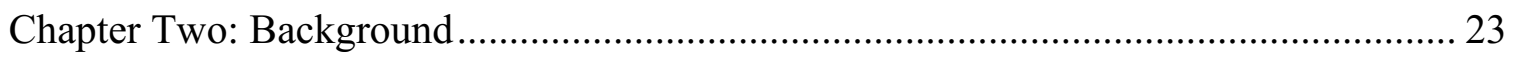

A Brief History of Sign Languages in Canada ............................................ 24

The Suppression and Recognition of Sign Languages in Canada ...................... 25

The Acceptance of ASL as a Foreign Language ........................................... 28

The Rise of ASL as a Foreign Language ..................................................... 34 
Ongoing Debates About Teaching ASL to Hearing Adults................................ 36

The Establishment of ASL Teachers' Associations............................................. 38

Common ASL Classroom Practices............................................................... 43

ASL in Canadian Colleges and Universities................................................. 52

Chapter Three: Theoretical Tapestry and Literature Review ..................................... 56

The Teacher-Narrative Turn ........................................................................ 56

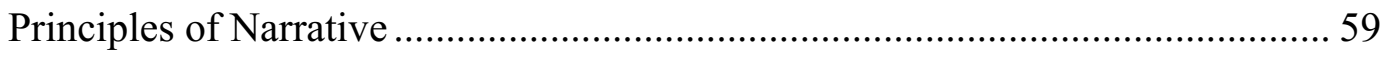

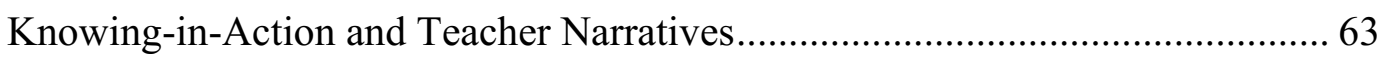

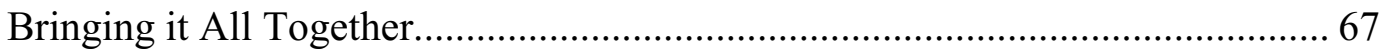

Review of Studies of ASL Teachers ........................................................... 70

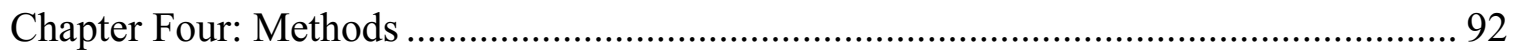

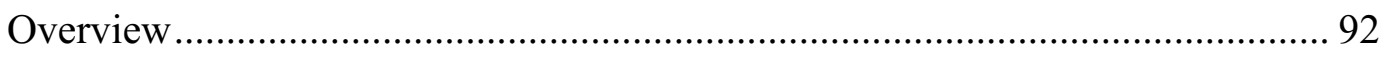

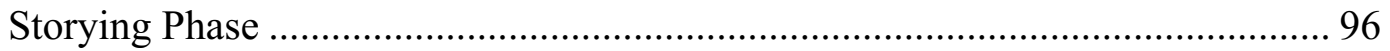

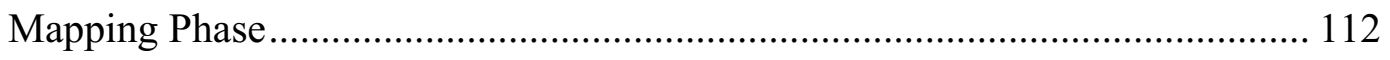

Validity Checks..................................................................................... 115

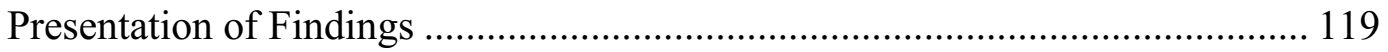

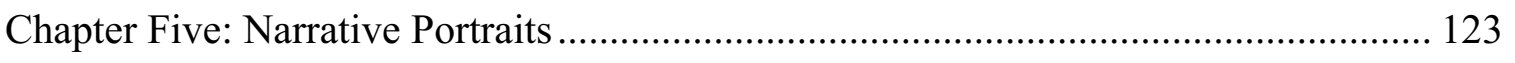

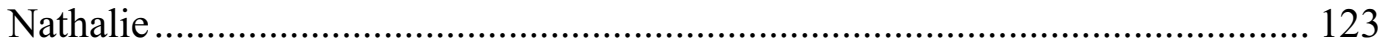

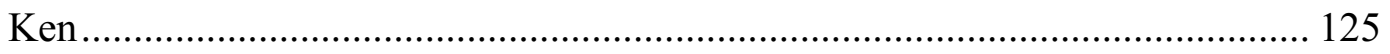

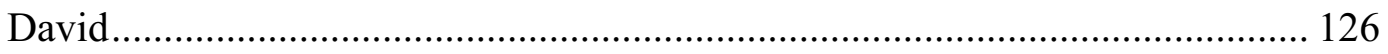

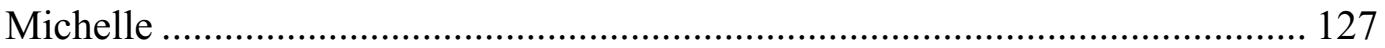

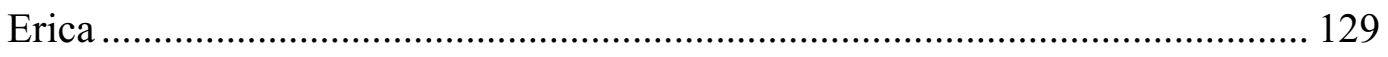

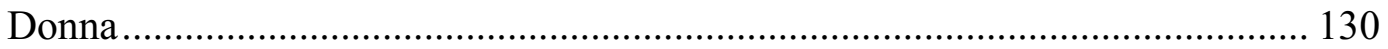


Nick

Chapter Six: Pathways to Teaching

Coming to ASL and the Deaf Community ........................................................ 134

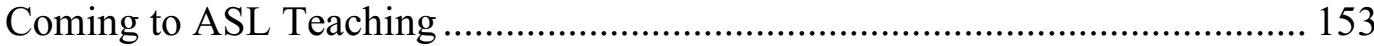

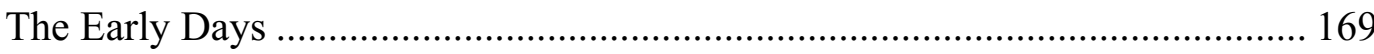

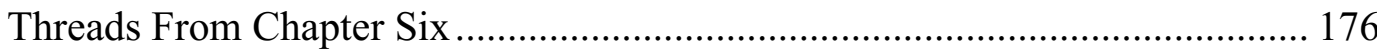

Chapter Seven: Pathways Through Teaching ……………………………………... 182

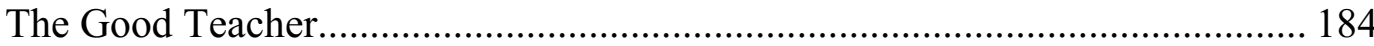

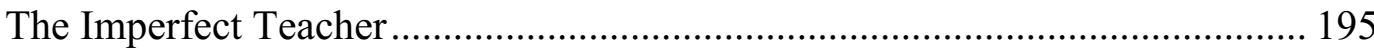

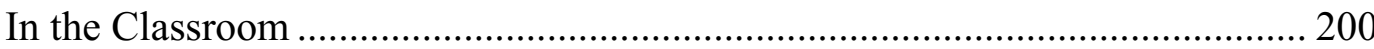

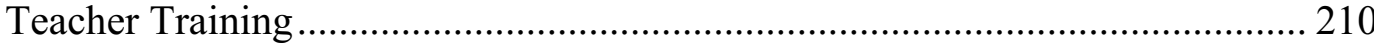

Collaboration and Community: Successes.......................................................... 219

Collaboration and Community: Challenges......................................................... 231

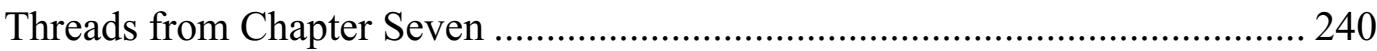

Chapter Eight: Pathways Forward ........................................................................ 244

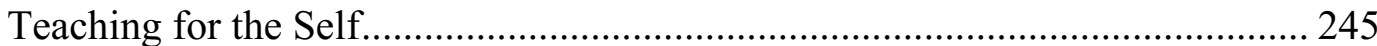

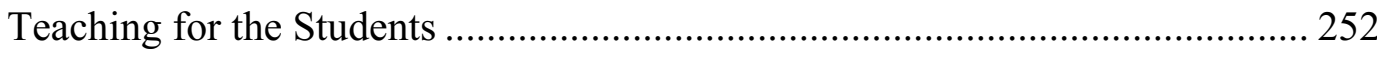

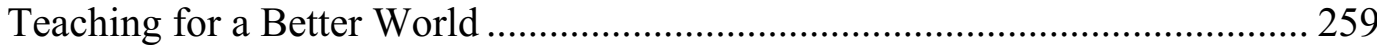

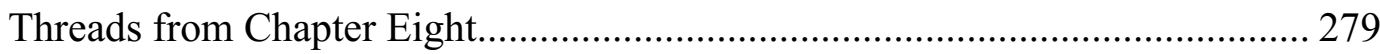

Chapter Nine: Pulling the Threads Together .................................................................. 281

Summary of Stories From Chapters Six to Eight................................................. 281

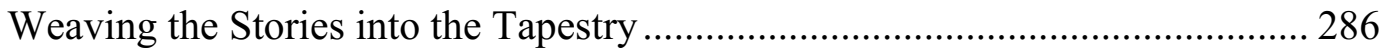

Potential Contributions and Recommendations ................................................. 296 
Chapter Ten: Reflections on My Research Journey …............................................. 302

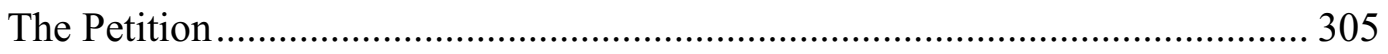

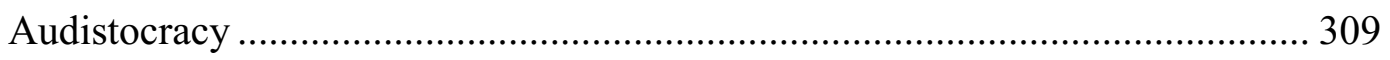

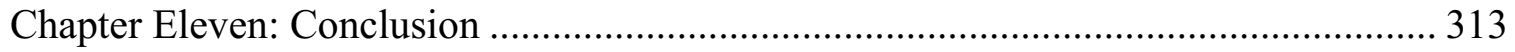

Limitations and Directions for Future Research........................................ 313

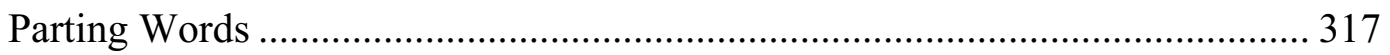

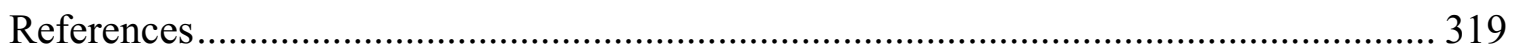

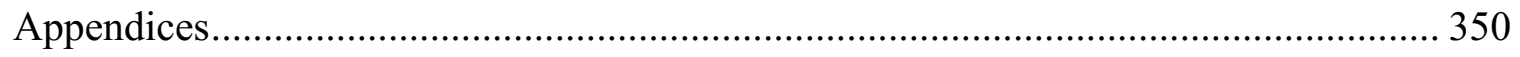




\section{List of Tables}

Table 1. Sum of Scores for ASL Teacher Knowledge/Skill Items (from Goertzen, 2018, p. 58) 86

Table 2. ASL Teacher-Participant Profiles ............................................................... 99

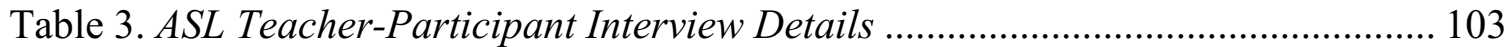




\section{List of Figures}

Figure 1. Theoretical relationship between stories, context, and literature.

Figure 2. Illustration of data collection, data analysis, and presentation of findings...... 93

Figure 3. Relationship between stories, threads, and contributions and recommendations. 


\section{List of Appendices}

Appendix A Summary of ASL Courses and Programs in Canadian Colleges and

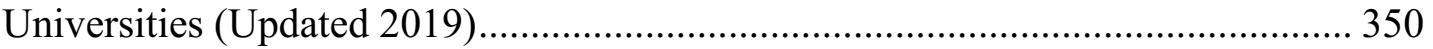

Appendix B Mock-up Sample Screenshot of Survey from Doré (2015)........................ 358

Appendix C Survey from Doré (2015) ……………...................................................... 359

Appendix D Follow-up Interview Guide from Doré (2015).......................................... 373

Appendix E CUREB Clearance Certificate \#106640 ...................................................... 374

Appendix F Invitation Email to Potential ASL Teacher-Participants ............................ 378

Appendix G Third-Party Recruitment Email for Snowball Sampling (ASL Teachers) 380

Appendix H Teacher Interview Question Guide ……………........................................ 381

Appendix I ASL Program Administrator Interview Question Guide ……………........ 383

Appendix J Sample Coding and Inter-Coder Reliability Check ..................................... 384

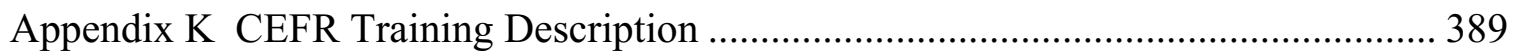

Appendix L Carleton University Petition: "Letter to Carleton University: ASL

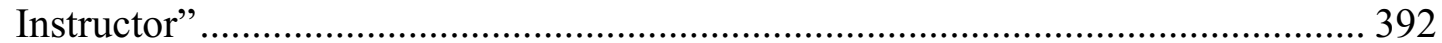




\section{List of Abbreviations}

ACSD Alberta Cultural Society of the Deaf

ADA Americans with Disability (Act)

AGB Alexander Graham Bell

ASL American Sign Language

ASLICE American Sign Language Instructor Certificate Evaluation

ASLPI American Sign Language Proficiency Interview

ASLTA American Sign Language Teachers' Association

CAD Canadian Association of the Deaf

CCSD Canadian Cultural Society of the Deaf

CEFR Common European Framework of Reference for Languages

CHS Canadian Hearing Society

CI Cochlear implant(s)

CTESL Certificate in Teaching English as a Second Language

DPN Deaf President Now

ELT English Language Teaching

ENT Ear, nose, and throat doctor

ISL Indigenous Sign Languages

K-12 Kindergarten to Grade 12

L1 First language

L2 Second language

LSQ Langue des signes québécoise (Quebec Sign Language) 
MAPSLI Maritimes Association of Professional Sign Language Interpreters

MCE Manually Coded English

MLA Modern Languages Association

MSL Maritime Sign Language

PSE Pidgin Signed English

SEE Signed Exact English

SIGN Sign Instructors Guidance Network (now the ASLTA)

SLA Second Language Acquisition

SLIC Sign Language Institute of Canada, also Sign Language Instructors of Canada

UNB University of New Brunswick 


\section{Glossary}

This glossary includes definitions for the key terms, concepts, and theories used in this dissertation. Many of these are subject to debate and discussion in and across disciplines, but within this dissertation I defined these terms, concepts, and theories according to the definitions provided below.

Alexander Graham Bell: Known in the deaf vernacular for his discrimination against deaf people, e.g., eugenicist policies against the intermarriage of the deaf and support for oralist deaf education.

Ameslan: The name for ASL prior to the 1960s.

Audism: The ongoing discrimination of deaf people by hearing people who consider deaf people inferior on the basis of their hearing (Ladd, 2005).

Categories: Patterns identified across codes to "consolidate meaning and explanation" (Grbich, 2007, as cited in Saldaña, 2013, p. 9); a methodological term.

Coda: Child of deaf adult/s.

Code: "A word or short phrase that symbolically assigns a summative, salient, essencecapturing, and/or evocative attribute for a portion of... data" (Saldaña, 2013, p. 3); a methodological term.

Cover Stories: Stories teachers tell about their experiences to make themselves look like experts, confident, in control (Clandinin \& Connelly, 1996); a type of teacher story.

Deaf Institute: A deaf school, day or residential (overnight); historically, key places for sign language transmission. 
Deaf President Now: A movement in the late 1980s where deaf people across the USA rallied to protest the instatement of yet another hearing president of Gallaudet University.

DEAF-GAIN: A concept reframes hearing loss to deaf gain; embraces a diversity perspective, where deaf people and their associated languages and cultures form a natural part of human biocultural diversity (Bauman \& Murray, 2014).

deaf (lowercase): A person who is unable to hear sound; may or may not use sign language or identify with a deaf culture.

Deafened: A person who became deaf later in life, before or after having acquired a first, usually spoken, language.

Dialectic: The view that teachers' practices are informed by theory, and that practice itself generate theory (e.g., Connelly \& Clandinin, 1990).

Foreign Language: A field of study generally focused on language learning abroad, i.e., where the language being learned is not the common language of the community; e.g., learning Russian in Canada.

Gallaudet University: A private university in Washington, D.C. established for deaf and hard-of-hearing students.

Gloss: A system of writing ASL into English.

Grand Narrative: Meta-narratives that are not attributable to a single person but rather reflect discourses of the powerful.

Hard-of-Hearing: A person with some degree of hearing loss; may use hearing technologies (e.g., hearing aid, cochlear implant). 
Hearing Hegemony: The dominance of hearing people over deaf people; related to audism.

Hearing: A person who can hear; usually uses spoken language as a primary means of communication.

Knowing-in-Action: A theory that states that teachers learn or know through practice (Schön, 1983); related to the reflective practitioner.

Language Lives: A language teacher's prior learning, using, and teaching of languages (Ellis, 2018).

Mainstreaming: The integration of deaf or hard-of-hearing students into "hearing" schools.

Manualism: A mode of deaf education that uses sign language as the primary mode of instruction.

Models of Deafness: Ways of framing deafness; e.g., cultural/linguistic minority model, medical/disability model.

Modern Language: A more up-to-date designation for foreign language.

Narrative Inquiry: The study of narratives and narrative practices.

Narrative Unit: A complete story, a coherent thought that was introduced, described, and concluded, and where further deconstruction would mean losing essential meaning. Narrative: The object of narrative study; also, stories.

No-Voice Rule: A common rule in ASL classrooms that disallows students to use their voice.

Oral Deaf: Person who is deaf but who uses primarily spoken language (lip reading, speaking, etc.); may or may not identify with a cultural deaf identity. 
Oralism: A mode of deaf education that uses spoken language as the primary mode of instruction.

Personal Practical Knowledge: A “teacher's knowing of the classroom” (Connelly \& Clandinin, 1988, p. 25) that is "shaped by the personalities of the teachers themselves, their past experiences, and how they view teaching" (Calderhead, 1996, p. 718).

Phonocentrism: Based on prioritization of spoken over written language; i.e., the belief that sign language is not an adequate substitute for spoken language, inferior.

Privilege: “[T]he other side of oppression” (Ferguson, 2014); e.g., hearing privilege, audiocentric privilege, able-bodied privilege.

Professional Development: An umbrella term to encompass a wide range of formal (e.g., workshops) and informal (e.g., "hallway chats") activities intended to develop teachers' knowledge, skills, and attitudes.

Reflective Practitioner: A teacher who learns about teaching through practice and reflection on practice (Schön, 1983); related to knowing-in-practice.

Restorying: The process of interpretation and reinterpretation of stories in narrative inquiry (Clandinin \& Connelly, 2000).

Sacred Stories: Stories of teaching that identify the "right" and "wrong" ways to be/do/know as a teacher (Clandinin \& Connelly, 1996); a type of teacher story. Second Language: A field of study generally focused on language learning that occurs within the community where the language is commonly used; e.g., English or French learning in Canada. 
Secret Stories: Stories about teaching that are modified or untold so as to maintain the face of a "good" teacher, to not disturb the sacred story (Clandinin \& Connelly, 1996); a type of teacher story.

Sign Language Community: Community that includes “" [s]ign language interpreters, hearing parents and siblings, ex-oralists, and possibly non-Deaf who are Deaf educators, and signing Deaf" (Eckert, 2010, p. 325).

Signing Naturally: A highly popular deaf-authored ASL textbook series.

Sociality: The personal (intrapersonal) and social (interpersonal) interactions in a story; one of the three dimensions of narrative analysis (Clandinin \& Connelly, 2000).

Space: The spaces and places in a story; one of the three dimensions of narrative analysis (Clandinin \& Connelly, 2000).

Teacher Learning: The complex process of growth and development across many dimensions (knowledge, skills, emotional, personal, etc.) through lived experience in and out of the classroom (e.g., Elbaz, 1981; Elbaz-Luwisch, 2005).

Teacher Stories: Stories teachers tell about teaching; see also sacred, secret, and cover stories.

Technical Rationalism: A dominant view in education that holds that knowledge is objective, exists outside of the body and the social world, and can be transmitted from one person, a teacher, to another, a student (Johnson, 1987, as cited in Clandinin \& Connelly, 2000).

Temporality: The continuity across time in stories; one of the three dimensions of narrative analysis (Clandinin \& Connelly, 2000).

Theme: Overarching patterns in data; methodological term. 
Thread: Overarching patterns in narrative data; methodological term evocative of storytelling language.

Training: Discrete, organized teacher professional development activities that target specific theoretical or practical knowledge and skills development with some degree of standardization; e.g., workshops, certificates.

Transformative Intellectual: Individuals who, through collective efforts, can be a major force for social good (Giroux, 1988).

Unit of Analysis: The smallest segment of data that can be used in analysis, where further segmentation would result in losing the qualities or meaning of the whole (Vygotsky, 1962/2000, as cited in Moen, 2006); see also narrative unit. 


\section{Chapter One: Introduction}

I mean, we're teaching the language but it's more than that, right? It's more than that. If I can change my students' views, their attitudes, maybe others will see that. There's power there. The more people the better to join the cause, to solve problems. Hopefully, yeah, hopefully teaching hearing students will bring more voices to the debate. People will pay more attention to some of these ongoing issues. That's why I teach. I'm teaching the language and a little bit about the culture.

The above quote was drawn from an interview I conducted with Donna (a pseudonym), an American Sign Language (ASL) teacher who participated in this dissertation study. Donna had spent the past 30 years as an ASL teacher for hearing adult learners and was a leader in her local deaf cultural organization. Deaf herself, she aimed in both roles to nurture hearing people's respect and appreciation for sign languages, deaf people, and deaf culture in Canada. I chose Donna's reflection above to open this dissertation because it illustrates how sign language teaching has deeply rooted connections to deaf culture and how ASL teachers like Donna may take on the roles of linguistic and cultural guides as well as deaf advocates, spokespeople, and allies. Donna's words are a reminder that ASL teachers are, intentionally or not, at the front lines of an ongoing movement to foster a more equitable society. Despite these important roles of ASL teachers, little research has considered who they are or what their experiences have been in navigating their work. This dissertation responds to that gap.

The academic literature reviewed for this dissertation arguably paints a somewhat unflattering portrait of ASL teachers who work in this instructional context-i.e., 
teaching ASL to hearing adult learners. For instance, it has been documented that teachers are often hired with little experience and background in language teaching in order to meet the growing demand for courses (Cooper, Reisman, \& Watson, 2011; Smith, 1988). Likewise, teachers' awareness and participation in formal training opportunities (e.g., workshops, professional development programs) was found to be ad hoc (Doré, 2015). The lack of standardization amongst teachers in terms of qualifications and teaching practices has prompted calls for more and more rigorous and standardized training programs and qualification requirements before teachers step foot into the classroom (Battison \& Carter, 1981; Jacobowitz, 2007; Kanda \& Fleischer, 1988; Newell, 1995abc; Scheetz \& Martin, 2006). While findings such as these are arguably valuable for directing efforts at growth and innovation, they rarely celebrate teachers and their diverse backgrounds and experiences.

In general, literature about ASL teaching and its teachers is lacking (McDermid, 2005; Quinto-Pozos, 2011; Thoryk, 2010). Briefly, Quinto-Pozos (2011) and Rosen (2010) proposed that sign language research has its roots in theoretical linguistics and this trend has carried through to today, leaving applied sign linguistics underdeveloped. Quinto-Pozos (2011) summarized the state of the art:

Notwithstanding its popularity as a language, it seems to be the case that, over the years, there has not been substantial dialogue between ASL teaching professionals and educators and researchers from other foreign language units. There are few journal articles that can be found concerning ASL pedagogy, models and theories of second language acquisition (SLA) and language teaching have primarily focused on the teaching of spoken and written languages, and SLA-focused 
conferences have mostly not included representation of the teaching of ASL or other sign languages. (p. 138)

Despite the popularity of ASL among hearing learners since the 1980s, academic investigation related to ASL teachers and their professional practice is limited (Kelly, 2001; McDermid, 2005; Quinto-Pozos, 2011; Swaney \& Smith, 2017). Cooper et al. (2008) observed that "no comprehensive professional journal exists to serve the discipline of sign language instruction" (p. 78) but such a journal is not yet warranted, as much of the relevant data on ASL pedagogy is neither published nor derived from empirical study.

As the demand for ASL instruction continues and ASL becomes more established, particularly in the higher education landscape where ASL is frequently offered, it is increasingly important to build a solid research base that considers ASL teachers' lived professional experiences. Such research could provide support for professional development efforts and networks that "guide entry and facilitate advancement within the field" (McDonald, 2011, p. 1).

In response, my doctoral research explored the professional life stories of ASL teachers in Canada whose learners were generally hearing (non-deaf) adults in postsecondary and community-based ASL courses and programs. I have spent the past eight years, which included a Master's in the same field, learning about and occasionally participating in the work that ASL teachers do. What became apparent to me early on in this work was that existing academic research about the profession did not reflect the intensity and importance of the activities that teachers undertake, individually and collectively, formally and informally. I thus oriented my doctoral research towards 
understanding ASL teachers' entry to and experiences with their work, including their skills and knowledge development, how they conceive of their roles as teachers, and what their hopes are for their learners, themselves, and the various linguistic and cultural communities of which they are a part.

In order to achieve this research goal, I conducted multi-part interviews with seven ASL teachers to understand their experiences_-or pathways (McDonald, 2011) coming to and moving through their profession. To better understand the context that constrained and supported their practice, I also conducted interviews with other stakeholders (representatives from deaf cultural organizations and ASL program administrators) and collected publicly available data about ASL in Canada. I analyzed the data narratively (Clandinin \& Connelly, 2000), informed by a collection of theories about the nature of narrative and narrative knowing in teachers' lives (e.g., Clandinin \& Connelly, 1996, 2000; Connelly \& Clandinin, 1988; Elbaz, 1981; Elbaz-Luwisch, 2005; Lave, 1993; Schön, 1983), which is described in depth in Chapter Three.

Before introducing further details of the study that is the focus of this dissertation, I would like to introduce myself.

\section{Self-Introduction}

I am a hearing, second language ASL user with a handful of deaf and signing friends but otherwise few ties to the deaf and sign language communities. I came across ASL by chance when I was completing an undergraduate degree in fine arts. One day between classes, I saw a poster advertising pay-what-you-can ASL courses. I had already studied several languages by then (with varying degrees of success) and I was intrigued by a visual language, and so I signed up right away. Ten years later, I am still learning. 
I started this Ph.D. as an applied linguist interested in language teachers and with several toes dipped into deaf studies. In the last year of writing this dissertation, however, I started a full-time job in educational development at my university, which I have come to learn has been a strong undercurrent in my Ph.D. work all along. In my current educational development role, I spend a lot of my time in conversation with instructors about teaching and learning in higher education. I learn about their learning outcomes and lesson plans, but also about their inner worlds as teachers - their motivations, ideas, hesitations, successes, goals, and so on. The listening and relational work came easily, as I am also a teacher by nature and by vocation: I worked for three years as an English teacher in community and private courses, and nearly eight years in my university as a teaching assistant, peer mentor, and instructor in my discipline. I also come from a family of several generations of educators, including my spouse, and so I care deeply about teachers on a personal level as multi-faceted professionals and human works-in-progress. All of this informed my interest in understanding the different professional journeys of the ASL teachers who participated in this study and their own unique backgrounds and goals.

The research questions that guided this dissertation are presented next.

\section{Guiding Research Questions}

In this dissertation I was guided by three overarching research questions:

1. What are ASL teachers' pathways to the profession?

2. What are their pathways through the profession?

3. What are their pathways forward in the profession? 
My research questions had a distinct and intentional chronological focus, as they considered teachers' professional lives to begin from their earliest experiences with the language, continue in their present experiences, and influence how they view their future. I was guided by Burns' (1999) proposition that attending to teachers' backgrounds helps to understand their "personal theories of teaching and learning" (p. 138), some of which develop early on in life. Ellis (2018) argued that language teachers' identities, practices, and professional development were shaped in part by their prior language experiences, what he referred to as their language lives, which included their prior learning, using, and teaching of languages. Attending to the early stages of teachers' pathways as well as their ongoing and future pathways was thus important in order to understand their later experiences.

I borrowed the imagery of pathways from educational developer Jeanette McDonald (2011; McDonald \& Stockley, 2008), whose work was about the experiences, relationships, processes, events, chance, and circumstance that led educational developers to their current profession. I also looked to the complementary imagery of landscapes, drawing from Clandinin and Connelly’s (1996) professional knowledge landscapes, which explained how teachers were accountable to the demands of multiple contexts, in and out of the classroom. In other words, teachers' ongoing experiences (or pathways) were shaped — whether enabled or constrained—by the conditions of their surroundings (or landscapes), including the prevailing educational policies of the time, available teaching resources, the state of pedagogical research, the evolving demands of their students, and so forth (Clandinin \& Connelly, 1996). These and other influences on my research are discussed in brief later in this chapter and in detail in Chapter Three. 
In order to clarify for the reader some of the concepts I use throughout this dissertation, it might be be helpful to define several key terms below. These terms have also been included in the Glossary.

\section{Clarification of Terminology}

There are several terms I used throughout this dissertation that required more explanation than could be provided in the Glossary. The latter terms from deaf studies may be particularly informative for those unfamiliar with the field.

\section{Narrative, narrative inquiry}

As described in Chapters Three and Four, narrative inquiry was the primary methodology used in this dissertation study. In adopting a narrative methodology, I experienced several points of discomfort with the terminology, particularly to describe the study's methods and outcomes. More specifically, as I moved through the writing of this dissertation, I found it difficult to balance terminology from narrative inquiry with the language of the "traditional" academic dissertation in applied linguistics where the readers would not solely be narrative inquirers. Traditional methodological terms such as unit of analysis, data, and data analysis were among those that did not wrap themselves fully around my intended tone and aims, and yet I knew that narrative terms such as restorying (Clandinin \& Connelly, 2000) and narrative units would require significant signposting and clarification for readers unaccustomed to them. In response, I mainly used the "traditional" terminology of academic dissertations to structure the dissertation ("Introduction", "Background", etc.) and reserved narrative terms for the body of the text.

To clarify, as per Connelly and Clandinin (1990), I use narrative to describe both the object of narrative study (or stories) and the study of narratives and narrative 
practices (or narrative research or inquiry). I refer to storytelling as the narrative practice of telling stories, and restorying (Clandinin \& Connelly, 2000) to refer to the process I underwent in re-telling and drawing meaning and connections from the study's participants' stories.

\section{Teaching as a profession}

Throughout this dissertation, I refer to ASL teaching as a profession and its teachers as professionals, yet in the literature of professions, teaching is often considered a semi- or quasi-profession, if at all (Talbert \& McLaughlin, 1994; Whitty, 2000). In brief, Talbert and McLaughlin (1994) asserted that teachers have a weak technical culture as a result of not widely sharing their knowledge about practice and therefore they cannot claim the collective strength of full professions. Welker (1992, as cited in Sato, 2013) argued that, unlike doctors and lawyers, teachers pass on their knowledge to their learners ("clients"), which casts doubt on the degree of specialized knowledge and skill required for the task. From English language teaching (ELT), Breshears (2004) claimed that ELT's ongoing lack of a defined body of knowledge precluded professional status.

Still, in this dissertation, I consider ASL teaching a profession. As Ingersoll and Collins (2018) argued, "upgrading the teaching occupation [to a profession] will lead to improvements in the motivation, job satisfaction, and efficacy of teachers, which, in turn, will lead to improvements in teachers' performance, which will ultimately lead to improvements in student learning" (p. 211, italics added). I add that a status "upgrade" (to borrow Ingersoll and Collins' term) to profession could also lend coherence to a scattered group of workers and perhaps help ongoing efforts at supporting their work. 


\section{Training, professional development, teacher learning}

Three related terms I use in this dissertation are professional development, training, and teacher learning. Academic discussions regarding the meaning and interchangeability of these terms are extensive; however, these discussions largely occur within the context of educational research about K-12 (kindergarten to Grade 12) teaching where there is a clear, pre-defined educational pathway to and through the profession-higher education (e.g., B.Ed. or M.Ed.) followed by pre-service training (e.g., placement) and then in-service training (professional development). In the K-12 teaching context, professional development (or $P D$ ) and training have been used synonymously to refer to mandated, standardized activities that seek to improve teachers' knowledge, skills, and practices for improved student outcomes (Opfer \& Pedder, 2013). Teacher learning, meanwhile, has been used to refer to "a complex and recursive process embedded in lived experiences in classroom practices" (Opfer \& Pedder, 2011, as cited in Ouellette, 2021, p. 82). Several scholars have pointed to the tensions that can arise when teachers' learning experiences do not align with or inform professional development as defined above (e.g., Hainer-Violand, 2013; Webster-Wright, 2009).

In this dissertation I do not use these distinctions. This study's teacherparticipants worked in post-secondary and community-based ASL programs where the curriculum and expectations regarding teachers' background, knowledge, skills, and credentials were established locally, if at all. Currently, there is a Canadian national sign language teachers' association that offers some training opportunities (see Chapters Two and Three), but teachers' participation in these activities is difficult to enforce, nor is there a widely recognized centralized body regulating their knowledge, skills, or 
practices. Thus, the above distinctions about teacher learning and development did not readily map onto my context of interest. Instead, I developed my own understandings, which I describe next.

In this dissertation I use professional development as an umbrella term to encompass a wide range of activities that I group into two categories: formal and informal. I use formal professional development synonymously with training to refer to discrete, organized activities that target specific theoretical or practical knowledge and skills development with some degree of standardization, such as workshops, presentations, conferences and learning institutes, and training and certificate programs (usually with a fixed curriculum and schedule that extends over multiple sessions). For example, the ASL teacher-participants in this study engaged in formal professional development activities such as completing the ASL teacher certificate program (see Chapters Two and Three) and participating in workshops and presentations by scholars about teaching particular linguistic features of ASL (e.g., classifiers) or adapting the Signing Naturally textbook series to Canadian ASL classrooms (see Chapter Seven, in particular).

The second category, informal professional development, includes ongoing, openended activities, including self-directed activities like action research, independent materials development, and engagement with academic literature (research papers, conferences), and collaborative activities like peer observation and feedback, mentorship, and participation in professional associations (Harmer, 2007). For example, the ASL teacher-participants in this study engaged in informal professional development activities 
such as sharing teaching materials, "hallway talks" with colleagues about teaching and learning, and self-reflection (see Chapters Six to Eight).

Like Ouellette (2021), I use teacher learning to refer to the complex process of growth and development across many dimensions (knowledge, skills, emotional, personal, etc.) through lived experience in and out of the classroom (Elbaz, 1981; ElbazLuwisch, 2005). In this research, my assumption is that teacher learning happens through participation in all forms of professional development, although the learning that occurs may or may not align with the intended learning outcomes of formal activities. Examples of the study's ASL teacher-participants' learning through experience, or their knowingin-action (defined below; see also Chapter Three) can be found across all of their stories and are discussed in more depth at the end of Chapters Six to Eight and in Chapter Nine.

\section{ASL as a foreign language}

Another term to clarify is my classification of ASL as a foreign language. The status of ASL as foreign has been contested in the literature; still, I use it in this dissertation for two reasons. First, calling it foreign helps to differentiate my context of focus-i.e., ASL instruction for hearing adult learners in mainly post-secondary and community-based (i.e., adult education) settings - from deaf education contexts where ASL is taught as a first or second language to deaf children. Second, although the learners in my context of study were second language learners of ASL (i.e., they had already acquired a first, usually spoken, language), I do not refer to ASL as a second language, as I do not speak to second language literature that tends to focus on topics such as second language acquisition and bilingualism. Arguably, I do not speak to foreign language literature either, which is a well-established field of study generally focused on 
language learning abroad (i.e., where the language being learned is not the common language of the community). However, my calling it foreign is a nod to the long history of ASL scholarship that has pushed for institutional recognition of the cultural and linguistic relevance of ASL and its acceptance in the post-secondary foreign language curriculum (e.g., Armstrong, 1988; Cooper, 1997; Corwin \& Wilcox, 1985; Gray, 2018; Jacobowitz, 1999; Miller, 2008; Olsen, 1988; Peterson, 1999; Peyton, 1998; Reagan, 2000; Rosen, 2008, 2015; Wilcox, 1988, 1989; Wilcox \& Peyton, 1999; Wilcox \& Wilcox, 1991; to name a few). This history is described in depth in Chapter Two. Perhaps in future studies when applied sign linguistics is more established I will refer to ASL as a modern language or as something else entirely.

\section{d/Deaf}

A recurrent term used in this dissertation is deaf, as many of the participants in my study were deaf. In much of the literature about sign languages and their users, it is common practice to use lowercase deaf to refer to people with limited hearing who do not use sign language or identify themselves as members of a community, and uppercase Deaf to refer to members of a cultural group that uses ASL and shares cultural values and beliefs (Wilcox \& Wilcox, 1991). It is not unproblematic to distinguish the two terms in this way nor is it universal to do so. I opted not to use the $d /$ Deaf distinction. As Napier and Leeson (2015) observed:

... [G]iven the complexities of sign language transmission, the evolving nature of the deaf community due to medical interventions and changes in educational policy, greater numbers of deaf people come to the community as late learners [of 
sign language]... Thus, definitions of deaf community membership are changing. (p. 2)

The deaf/Deaf binary overlooks the significant body of research in deaf studies about the diversity among deaf people linked to such things as use of spoken/sign language and use of hearing devices such as cochlear implants and hearing aids (e.g., Marschark, Zettler, \& Dammeyer, 2017). Kusters, de Meulder, and O’Brien (2017) argued that d/Deaf is outdated given the growing body of research on deaf identities as multiple and shifting. At times in this dissertation, I combine terms like deaf/hard-of-hearing and deaf/oral deaf for teacher-participants who self-identified in several ways throughout our conversations to reinforce that these identities can indeed exist in parallel or on a continuum.

Moreover, the use of upper-case Deaf as innately linked to sign language use inaccurately suggests only deaf people are sign language stakeholders, yet the sign language user base comprises a significant portion of non-deaf/hearing people (Snoddon \& Wilkinson, 2019), including native users (often children of deaf adults, or codas; see Preston, 1992, for a notable ethnography of coda identity) and second language users such as interpreters and parents of deaf children who have long been an integral part of the community. Sign language learners also represent the next generation of hearing signers.

Ultimately, $d /$ Deaf is historically preferred, and so I retained the lowercase/uppercase distinction when citing authors who use it in their writing. In my own writing, however, I opted for less charged terms to avoid imposing identities and affiliations on the teacher-participants in this study. I use deaf and hearing to indicate 
medical hearing status, and, when applicable, I indicate whether the individual uses sign language as a primary/secondary or first/second language and if they identify with a deaf culture or a deaf community. My reference to deaf communities, of which there are many, broadly refers to a group of individuals who share a core culture, including using and valuing sign languages, though their deaf/hearing/other statuses and other aspects may vary. Likewise, I use deaf culture to refer to that core culture with which many people, deaf and hearing, identify, but I also refer to sign language communities, which I consider to include core sign languages users for whom sign language is central to their daily lives, including deaf and hearing native signers, sign language interpreters, and signing family members of deaf people, as well as more peripheral users, such as novice sign language learners.

\section{Audism, phonocentrism, models of deafness}

Two concepts from deaf studies that I use recurrently in this dissertation are audism and phonocentrism, both of which relate to different models of deafness. Audism describes discrimination on the basis of hearing (first used in Humphries, 1977). Phonocentrism refers to the prioritization of spoken language and sound over sign languages and visual communication based on "the historical assumption that speech is the most fully human form of language" (Bauman, 2004, p. 243). Together, "[p]honocentrism explains the origin of audism, and audism explains the rationale for the sociopolitical manifestation or institutionalization of audism in hearing colonization of deaf people" (Fernandes \& Shultz Myers, 2009, p. 30). The concept of deaf people as colonized originates from Lane's (1992) comparison of deaf people and the formerly Dutch-colonized Burundians. Lane found many points of similarity, including 
paternalistic attitudes and policies by the colonizers, particularly around medical (hearing aids and cochlear implants) and educational issues (oralism or Signed English over ASL), and the cultural identity conflicts these provoked.

Audism, phonocentrism, and deaf (de)colonization were not the main focus of this dissertation but were certainly embedded in the lived realities of many of the teachers who participated in this study. The concepts appeared in teachers' stories about their experiences of being told—or telling themselves—what deaf people can do (or DEAF CAN in ASL gloss, i.e., writing ASL into English) and cannot do (DEAF CAN'T). O'Brien and Placier (2015) identified DEAF CAN discourses as those that associate deafness with deaf cultural values, deaf pride, and a sense of deaf belonging and identity; DEAF CAN'T are discourses that portray deaf people as having a medical pathological condition or disability and as lesser than hearing people. These competing discourses, or models of deafness, reflect a long and rich history of scholarship that are worth briefly expanding here, particularly for those readers unfamiliar with deaf studies.

A disability model of deafness stems from the medical/pathological view that deafness is a dis-ability, i.e., a problem of ability to be resolved with cures and normalizing hearing technologies (e.g., hearing aids and cochlear implants) and oralbased education where sign language is limited or prohibited in favour of lip reading and speech (Edelist, 2015; Lane, 2002). The same mentality holds that if a child is taught to sign, it should be a sign-based system of English, or manually coded English (MCE; e.g., Signed English), which are "superior to ASL, because they [ ] present a model of spoken English on the hands" (Lane, Hoffmeister, Bahan, 1996, p. 269). 
Lane (2002) argued that the disability model of deafness was the root cause of the oppression and misrepresentation of deaf people by its implicit aim to eradicate (culturally) deaf people, which the audist mainstream considered a defective, troublesome, and costly group. At the same time, general disability advocacy and legislation around accessibility have had many positive impacts on deaf issues. The relationship between the disability label and deaf people is complex and beyond the scope of the present dissertation to explore in much depth. For further reading about the deaf-disability relationship, I recommend the reader consult Foster (2001) and Scott-Hill (2003).

To counter the discourse of deafness as a deficit or loss, Bauman and Murray (2014) led a counter-movement called DEAF-GAIN. At the heart of DEAF-GAIN is the aim to reframe the interpretation "deaf" from hearing loss to deaf gain. DEAF-GAIN embraces a diversity perspective that recognizes that deaf people and their associated languages and cultures form a natural part of human biocultural diversity. DEAF-GAIN advocates suggest that the persistence of deafness and sign language throughout the history of humankind points to naturally occurring variations on human biology and should be appreciated for the various ways that deaf people (intrinsically) and the world (extrinsically) gain from deafness. Komesaroff and MacLean (2006) developed a similar idea of "positive biology" to refer to "the skills and faculties Deaf people have developed through their biological difference" (p. 92).

The above terms have all been included in the Glossary for future reference. In the next sections I explain the various theoretical influences on this research.

\section{Theoretical Framework}


This research was informed by a range of theoretical and empirical literature, which I explain briefly in this section and again in more depth in Chapters Two and Three. First and foremost, this study, in particular its methodology, was framed by theories of teacher narratives from educational research that collectively accounts for how teachers tell stories to make sense of and learn from their lived experiences (e.g., Clandinin, 2007; Clandinin \& Connelly, 1996, 2000; Connelly \& Clandinin, 1990; Elbaz, 1981; Elbaz-Luwisch, 2005). I also looked to theories of professional learning (Lave, 1993) that take a dialectic view of the relationship between theory and practice, i.e., teachers' practice is informed by theory — formal and embodied or lived — that in turn helps to generate theory that feeds back into practice (Clandinin \& Connelly, 2000; Schön, 1983). Reflection through storytelling can thus both capture and promote professional learning.

Underpinning the above theories is a multi-disciplinary framework of narrative principles that states that storytelling is a form of social action and socially enabled and constrained performance (Chase, 2005; as described in depth in Chapter Three). Drawing out teachers' stories through narrative research is a way of illuminating not only the details of teachers' lived experiences, but also identifying features of the broader sociohistorical context to which teachers speak (e.g., align with, reject, modify) through their stories and their storytelling. The broader sociohistorical context of ASL in North America (Chapter Two) and empirical literature about the profession of ASL teaching (Chapter Three) are therefore particularly important in situating the present research. 
Having provided an overview of the theoretical framework of this research, below I briefly describe how the study that is the focus of this dissertation shifted over time and the potential contributions of this dissertation study in academic and practical terms.

\section{A Note on Methodology and Scope}

I had originally intended for this study to respond to a different set of questions that I would address in a different way. I entered my Ph.D. with the goal of exploring the wants and needs of Canadian ASL teachers in order to help inform an ASL teacher professional development program in collaboration with the national sign language teachers' association, the SLIC (Sign Language Instructors of Canada, under the umbrella of the Sign Language Institute of Canada). In the first year of my Ph.D., the SLIC and I conducted a preliminary needs analysis study (Doré, 2015; described in Chapter Two) and began planning for next steps, including identifying possible grants for professional development activities and updating the SLIC's internal structure. Nearly two years into the collaboration, however, the relationship collapsed for several reasons (discussed further in Chapter Ten) with no hope of being re-established. I redesigned the study on my own, shifting my emphasis from the SLIC and formal teacher professional development to focus on the lived experiences of ASL teachers themselves, i.e., where much of their professional learning took place, making them my primary focus and collaborators. The results of this re-envisioned research are presented in this dissertation, in which individual ASL teachers' professional life stories are highlighted as a way of understanding and supporting their practice. In the end, this was a more positive approach and a more direct way of supporting the teachers, which was always my ultimate aim. 
As I undertook this work, I was acutely aware of but spoke only peripherally to some of the larger intersecting conversations that surround ASL teaching, such as labour organization in higher education and the place of the part-time instructor, which most ASL teachers in Canada are, where employment is time-limited and often without longterm benefits or security. Likewise, I did not dive into discussions of the role of language education in the post-secondary curriculum more broadly, nor address scholarship around sign language second language acquisition or any of the technological innovations around sign language use (e.g., wearable technologies, artificial intelligence) that are currently shaping the linguistic landscape of sign languages. These issues are much larger than I could reasonably tackle in one dissertation and they did not feature heavily in most of the participating teachers' stories. Still, I touch on them at times throughout this work, including in the background chapter, Chapter Two, because it would be impossible to discuss ASL teaching without them.

With this scope in mind, I intended for this dissertation to create a larger space in academic conversations for ASL teachers' stories by focusing on their unique professional experiences and perspectives. It has been my feeling and observation that ASL teacher-focused research has been overlooked in favour of ASL learner-centred research (e.g., second language acquisition), where teachers were cast as a variable in student learning process rather than active, decision-making individuals on an ongoing professional journey all their own. I unpack this and other intended contributions of this dissertation below. 


\section{Intended Contributions}

Academic discussions have rarely focused on ASL teachers, much less featured their voices in matters of their own profession. Thus, looking forward, I hope that this dissertation will do exactly that: bring ASL teachers' voices into the academe in a way that they stand not as case studies supporting other scholarly ideas or ideals, but as stories of experience that in their own right advance knowledge in the field.

At the same time, I hope this research will encourage more dialogue and understanding among ASL teachers in Canada. Storytelling is a key way humans understand themselves, others, and the world around them (Clandinin, 2007). I hope that ASL teachers who come across this work will see themselves reflected in the stories in this dissertation and find meaning in them. I also hope that language program administrators of current and prospective ASL programs can glean some insights from this work in terms of understanding issues related to ASL teaching, and how the establishment and growth of ASL among hearing adult learners affects the sign language community.

Equally importantly, this research was intended to support the professional practice of the seven ASL teachers who participated in this study. Storytelling is a way to understand others, but also oneself through the telling (Conle, 2000; Polkinghorne, 1988; Schön, 1983), and so reflection on the past, present, and future can give teachers the opportunity to articulate and better understand and navigate their ongoing professional journeys. Our conversations were an invitation for the teachers to form theories on their own practice through self-reflection — and perhaps through my reflections, too. Reflection can also make space for teachers to appreciate how their work with a minority language 
like ASL has the potential to shape the world beyond the classroom walls; or, as Giroux (1988) would put it, teachers are transformative intellectuals capable of effecting positive social change through their work.

On a practical level, this research may fulfill its original aim of providing a foundation for planning for future ASL teacher learning, including for teachers individually as well as for their many collective networks, including the SLIC and other ASL teachers' associations, teacher-mentors, and program supervisors. The stories presented in this dissertation may be insightful of the kinds of experiences, wants, and needs that such networks can work together to address.

More personally, I am a friend and colleague of many ASL teachers across Canada. Through them I have seen, heard, lived, and re-lived stories of teaching and learning, identity and power, struggle and success — some of which I see reflected in the stories of this study's teacher-participants. These accounts have convinced me that there is perhaps an opening in the literature and in the profession for the sharing of stories that highlight the wealth and diversity of experiences within.

The final section of this chapter, below, provides a roadmap of this dissertation.

\section{Roadmap of the Dissertation}

In Chapter One, I have introduced the topic of the study and its primary aims and guiding research questions. In Chapter Two, I provide background about ASL, from its historical roots to its current status as a foreign language in Canadian colleges and universities. In Chapter Three, I situate this dissertation in theoretical and empirical academic conversations by reviewing a selection of theory about teacher narratives and literature about ASL teachers' professional lives. In Chapter Four, I outline the narrative 
methodology of this study and my decisions about the organization and presentation of findings.

In Chapters Five to Eight, I focus on the main findings of this research: the seven teacher-participants' professional life stories. In Chapter Five, I introduce the teachers who participated in this study through brief narrative portraits. In Chapter Six, I discuss the teachers' stories about their backgrounds with sign language and the deaf community and their early experiences as ASL teachers. In Chapter Seven, I move forward along the timeline and discuss the teachers' stories about their ongoing teaching experiences, including their teaching philosophy, practices, and relationships that have facilitated and impeded their work. I close the findings chapters with Chapter Eight, about teachers' reflections on their professional goals and the intended impacts of their work. I discuss key themes throughout Chapters Six and Seven, though a more expansive discussion is reserved for Chapter Nine.

To conclude the dissertation, in Chapter Ten, I reflect on my own research journey throughout this dissertation, and in Chapter Eleven, I consider the limitations of the study and propose directions for future research.

Having introduced the dissertation, I now turn to Chapter Two, which provides a condensed background of ASL and its teaching to hearing adult learners to help situate this dissertation in the broader sociohistorical context of sign languages in Canada. 


\section{Chapter Two: Background}

The teaching of ASL as a foreign language to hearing adults has a relatively recent and complex history. This chapter includes a roughly chronological history of the development and practice of ASL teaching, including:

- A brief history of sign languages in Canada

- Acceptance of ASL as a foreign language

- The rise of ASL in post-secondary institutions

- The establishment of ASL teachers' associations

- Common ASL classroom practices

- ASL in Canadian colleges and universities

I should preface this history by stating that all of it is framed within the broader historical context of deaf and sign language communities, which have faced many years of oppression by the dominant majority, intentionally or not. Deaf people may face discrimination based on their deaf bodies being perceived as deficient or lesser than bodies that hear fully (Hauser, O’Hearn, McKee, Steider, 2010; as described in Chapter One), and sign languages are dismissed as exaggerated mime or an accommodation or derivative of spoken language, not a "true" language (Lane, 1992; as described later in this chapter). These perspectives must be mentioned because they are deeply engrained in the fundamental realities of all of the teacher-participants in this study, whether they experience them firsthand or not. Consequently, the background presented in this chapter, beginning with a broad historical overview below, was an integral part of the analytical procedures of this study's teacher-participants' interviews because it provided essential social and historical context for their stories and my interpretation of them. 


\section{A Brief History of Sign Languages in Canada}

Like natural spoken languages, natural sign languages emerged and evolved over time with language contact through past and present international exploration, colonization, and globalization. Known until the 1980s as Ameslan or simply Sign prior to the linguistic breakthroughs of sign language linguist William Stokoe and his colleagues in the 1960s and 1970s (Stokoe, 1960/2005, 1978; Stokoe, Casterline, \& Croneberg, 1976), ASL is the result of language contact between Old French Sign Language, a precursor of today’s French Sign Language, and local sign, sometimes referred to as Old ASL (Wilcox \& Occhino, 2016). Old ASL had long been used prior to that point, the most oft-cited example being in Martha's Vineyard, Massachusetts, where a reportedly high rate of deafness meant hearing and deaf people alike signed as a matter of daily life (Groce, 1985).

ASL spread internationally to Canada and several countries across the world, influencing countless sign languages, some ways in which are only beginning to be studied formally (e.g., Miyamoto and Mori (2015) on Kenyan Sign Language as a sister language of ASL). In Canada today, ASL—rather, a dialect of ASL occasionally referred to as Canadian $A S L$, but usually simply $A S L$ — is the most commonly used sign language in the Anglophone sign language community, although accurate statistics on the number of users is difficult to ascertain (CAD, 2015). Quebec signers primarily use a close relative of ASL, Quebec Sign Language (LSQ; Parisot \& Rinfret, 2012), which was brought to Quebec deaf institutes (i.e., school, often residential) in the 1830s by Lower Canada lawyer Ronald MacDonald who studied under famed French deaf educator 
Laurent Clerc (Miller, 2001), the same Clerc who is credited with founding the first US American schools for the deaf (along with Thomas Hopkins Gallaudet).

Meanwhile, signers in Eastern Canada primarily use ASL, although many people of the older generations also use Maritime Sign Language (MSL), usually in combination with ASL. MSL is the result of early language contact between local Maritime signers and deaf Scottish and British immigrants in the 1800s (Warner, Doull, Falvey, Martell, McDermott, \& Richman, 1997). Although MSL has few users currently and is classified as a moribund language (Ethnologue, 2019), it has been the focus of some linguistics analysis (Yoel, 2009) and several ongoing grassroots revitalization efforts (Davie, 2019). Likewise, many Indigenous Sign Languages (ISL)—including, for example, Coast Salish Sign Language, Inuit Sign Language, Oneida Sign Language, Plains Indian Sign Language (including Blackfoot, Cree, Dakota, and Ojibwe), and Secwepemc Sign Language (Wolf, 2019) — are endangered and undergoing documentation and revitalization (Davis, 2007). The suppression of these languages, as described next, has played a role in their current status, although recent efforts at recognition and revitalization are currently underway.

\section{The Suppression and Recognition of Sign Languages in Canada}

As suggested in the introduction of this chapter, deaf communities have had a long history of resisting the suppression of sign languages and fighting to keep sign language alive, where it lives on as an essential part of deaf culture (Ladd, 2005). Deaf education has often been a target site for the eradication of sign languages because it is at the mercy of educational policy-makers who have alternately embraced and banned sign language use. 
When the first deaf school was opened in Canada in 1831, it was modelled after the manualist deaf schools in the USA, which by then had abandoned the early artificial manual English system for the natural sign language of the deaf community (Carbin, 1996). As deaf schools appeared across Canada, they continued to follow the USA model, including shifting from the two-handed British alphabet to the American singlehanded one (Carbin, 1996).

By the 1860 s, however, manualism was losing traction as oralism was increasingly favoured. By 1880, oralism "became the mandated policy in Canadian deaf education, and teachers and children were prohibited from signing in most classrooms" (Carbin, 1996, p. 322). Hearing teachers replaced deaf teachers and placed more emphasis on "articulation", i.e., speaking and speech reading (Carbin, 1996). With the rise of hearing aids and cochlear implants, many schools promoted the full or partial mainstreaming of deaf children in "regular" schools without or with little access to sign language. Mainstreaming has been referred to as an act of "symbolic violence" by reinforcing hearing dominance over deaf people (Branson \& Miller, 2009).

The intent to eliminate sign language use and for deaf children to assimilate into hearing culture came at the expense of high-quality education in their first natural (sign) language, resulting in lower achievement of deaf pupils compared to their hearing counterparts (Johnson, Liddell, \& Erting, 1989). Despite countless pleas and public demonstrations against oralism by deaf communities and organizations, oralism held fast — and largely continues to hold today.

It was not until scholarly advances in sign language linguistics in the 1960s and 1970s that signing made its way back into some classrooms (Carbin, 1996). However, the 
signing that was introduced was not always ASL or another natural sign language; instead, schools were promoting various artificial sign systems of manually coded English (MCE, e.g., Signed Exact English or SEE) or speech accompanied simultaneously by sign language vocabulary (i.e., simultaneous communication or simcom). The debate over what linguistic system—natural or artificial—belongs in the deaf classroom continues today.

There has been significant progress at legislative levels to improve the status of sign languages, particularly in deaf education. By 1993, Alberta and Manitoba had recognized ASL as the language of the deaf community and an optional language of instruction in K-12 and post-secondary, while Ontario amended its Education Act to recognize ASL and LSQ as languages of instruction (Carbin, 1996). While this recognition does not make manualism the de facto method for deaf education,

The provincial recognition of sign languages as languages of instruction in education may, however, strengthen the claims of parents of deaf children who want their children to receive instruction in sign language and may support the viability of deaf schools, which have played a key role in sign language transmission and maintenance. (Snoddon \& Wilkinson, 2019, p. 135) Indeed, deaf schools have been referred to as the "nucleus" (Carbin, 1996) and "the bedrock and the wellspring of the Deaf community" (Tucker, 1994/1996, p. 365), and so there is significant drive from deaf communities to keep them open.

Federally, the Accessible Canada Act (Bill C-81) that passed in May 2019 officially recognized ASL, LSQ, and ISL as "the primary languages for communication by deaf persons in Canada" (Bill C-81). This bill may be a step forward in improving 
deaf people's access to certain services in sign language, such as interpretation. At the same time, it couches sign language recognition firmly within an accessibility-i.e., disability-oriented —not a cultural-linguistic lens (Snoddon \& Wilkinson, 2019). This is problematic for language education, particularly deaf education (i.e., for deaf children) because it is positioned as an accommodation, and not as a valued part of the Canadian linguistic tapestry (Snoddon \& Wilkinson, 2019). Likewise, there are doubts about Bill C-81's ability to sway provincial deaf education policies, which are still overwhelmingly manualist (Snoddon \& Wilkinson, 2019).

The next sections trace the history and status of ASL in higher education, which has evolved significantly over the past 50 years.

\section{The Acceptance of ASL as a Foreign Language}

As mentioned previously, ASL was not widely recognized as a "real" languageeven by its own users—until the 1960s. For instance, a 1984 California Task Force rejected ASL as a foreign language, placing it among "computer languages", "artificial languages", and "dialects of English" (Chapin, 1988). The Modern Languages Association (MLA) similarly considered ASL an "invented language" alongside Star Trek's Klingon until as late as 1997 (Brueggemann, 2008). By the early 1960s and 1970s, however, the aforementioned advances in theoretical and sociolinguistics (e.g., Bellugi \& Fischer, 1972; Stokoe, 1960/2005; Stokoe, Casterline, \& Croneberg, 1976; Woodward, 1971) brought worldwide attention to the formal study of sign languages as legitimate languages. The UK and Scandinavia, for example, were quick to take up sign language research, but Canada did not begin to formally document its sign languages (structure, 
history, etc.) until the late 1970s (Carbin, 1996). These early delays to the establishment of an academic presence around ASL make its current popularity especially noteworthy.

The acceptance of ASL for foreign language credit is thus also fairly recent. Postsecondary institutions began to accept ASL for credit only in the mid- to late-1980s. A study by Corwin and Wilcox (1985) reported that in the mid-1980s, roughly $20 \%$ of colleges and universities in the USA accepted ASL for foreign language credit. A followup study in 1995 found that the number had risen to 50\% (Sinett, 1995). By 2004, more than $95 \%$ of language program administrators were in favour of its acceptance (Cooper et al., 2011), though this may vary between as well as within institutions (Sofinski, 2011). Recent numbers indicate that nearly 200 post-secondary institutions in the USA (Wilcox, 2019) and at least 30 in Canada (Doré, 2015) now offer ASL for credit.

The acceptance of ASL as a foreign language in higher education has grown significantly over the past few decades, but it was — and, by some accounts, continues to be (Jacobowitz, 2005) — hard-fought. A summary of nine scholarly arguments, mostly from the 1980s and 1990s when the status and academic value of ASL was being debated, is presented below. Arguments for and against ASL's acceptance as a for-credit foreign language are presented in conversation with each other in order to emphasize the complexity of the issue, which, in many cases, has been driven by audist beliefs (McDermid, 2009) and conflicting language ideologies rather than by evidence (Reagan, 2011). In the context of this dissertation, these arguments were embedded in the daily life of teachers, learners, administrators, the sign language community, and surely more, as part of a shared and ongoing history about the place of ASL in broader society. 
Below, note that I use "as cited in" to indicate that the argument is reported but not necessarily supported by the cited author/s.

- Argument 1: Sign languages are not real languages; they are pantomime (as cited in Baker \& Cokely, 1980).

- Response: This argument is founded in language beliefs and attitudes, not linguistic evidence (Reagan, 2011). ASL is a legitimate, natural language with a fully developed linguistic system (as evidenced in, for example, Klima \& Bellugi, 1979; Stokoe, 1978; Valli \& Lucas, 1993). ASL teachers should be sure to teach sign languages, not manual representations of spoken languages (i.e., MCE; Wilcox \& Wilcox, 1991).

- Argument 2: ASL is finitely expressive (Belka, 2001) and therefore "too easy" to qualify for academic credit (as cited in Jacobowitz, 2005 and Reagan, 2011).

- Response: ASL is classified as either a Category 2 or 4 language (of a possible 5; Albutt \& Ling, 2017), though difficulty should not disqualify a language from acceptance for foreign language credit, as difficulty depends on the learner's prior language learning experience, language aptitude, attitudes, and motivations, and similarities between their languages and the language being learned, to name a few.

\section{- Argument 3: ASL does not provide opportunities for linguistic comparison} for students to better understand their own language because of the absence of sounds and graphemes (Belka, 2001).

- Response: ASL learners have the opportunity to learn about their own familiar spoken language modality through contrastive analysis with a 
very distinct visual-gestural sign language (Chapin, 1988; Wilcox \& Peyton, 1999; Wilcox \& Wilcox 1991). As Davis (1998) noted: “There couldn't be a language more foreign to English. What could be more alien to people with normal hearing than that complex dance of fingers, hands, face and body which seems impenetrable to those who do not know ASL...?" (as cited in Pfeiffer, 2003, p. 36-37).

- Argument 4: ASL lacks a real cultural community (as cited in Reagan, 2011).

- Response: There are abundant publications outlining the unique features of deaf culture (see, for example, Baker \& Cokely, 1980; Padden \& Humphries, 1988; Reagan, 2000; Rutherford, 1988; Sacks, 1989), including how deaf individuals are bi-cultural, moving between hearing and deaf cultures, as a way of surviving in a phonocentric world (see, for example, Leigh, 2009; Valentine \& Skelton, 2003)

- Argument 5: ASL is not "foreign" because it is used in the same geographical context where it is taught (Belka, 2001; as cited in Sinnett, 1995; Wilcox \& Peyton, 1999).

- Response: The same holds true for Indigenous languages, suggesting it is more a question of naming rather than legitimacy of for-credit foreign languages. Foreign language departments are beginning to rename themselves second language or modern language programs.

- Argument 6: Deaf culture is not a unique culture but rather a sub-culture of mainstream hearing culture based on a disability, one of several "different manifestations of the diverse American culture" (Belka, 2001, p. 50). 
- Response: ASL is associated with American deaf culture, which is defined by sign language use and shared values, customs, and literature (e.g., folklore, jokes, theatre; Rutherford, 1988; Wilcox, 1999). ASL learners benefit from developing cross-cultural awareness (between mainstream hearing and minority deaf cultures) and communication skills (Smith, 1988; Wilcox \& Peyton, 1999; Wilcox \& Wilcox, 1991), particularly in relation to a culture that is "among us, though typically invisible to hearing persons. Indeed, the very invisibility is itself a strong argument for education and enlightenment of members of the majority culture" (Chapin, 1988, p. 112).

- Argument 7: Sign languages do not have a writing system, which is a main motivator for learning a foreign language (Belka, 2001; as cited in Reagan, 2011).

$\circ$ Response: There are many writing systems to represent ASL, though none are widely used (Wilcox \& Peyton, 1999). Many spoken languages are oral languages without a writing system, which does not detract from their legitimacy or value (Reagan, 2011).

- Argument 8: ASL does not have a literary tradition worth studying because as a language is too young and has too few users (Belka, 2001).

○ Response: Despite not having a widely used or easily accessible written system, there is a rich literary arts and performance culture associated with ASL, including oratory, folklore, poetry, plays, videotaped and written English auto-/biographies about/by deaf people (Frishburg, 1988; Wilcox 
\& Wilcox 1991; Wilcox 1999). These can expose hearing ASL learners a "new, different mode of aesthetic expression" (Chapin, 1988, p. 112).

- Argument 9: Sign languages and sign language professions will soon become obsolete because spoken language will be accessible to deaf people with cochlear implants and other hearing technologies, and so they can assimilate into hearing life and society (as cited in Krausnecker, 2015 and Mitchell, Young, Bacheleda, \& Karchmer, 2006).

○ Response: By learning about deaf culture alongside ASL, learners develop a pluralistic, diversity-oriented (linguistic, cultural) rather than an assimilatory, deficiency-oriented (disability accommodation, hearing loss) perspective of ASL and deaf people (Bauman, 2008; Rutherford, 1988; Wilbers, 1988). There are also many opportunities for learners to work with and immerse themselves in the DEAF WORLD (Kelly, 2001), including as interpreters, counselors, teachers of the deaf, and other such professions. As deaf activist and writer George Veditz said his 1913 National Association of the Deaf film Preservation of the Sign Language: "As long as we have deaf people on earth, we will have signs" (Veditz, 1913).

In light of the many misconceptions about sign languages and deaf people, ASL courses and textbooks reportedly spend a significant amount of time at the outset dispelling myths and promoting the legitimacy of sign languages as natural languages (Calton, 2013). Some common ASL classroom practices are described later in this 
chapter. Next, I describe how, despite a slow start, ASL has since become wellestablished as a foreign language in post-secondary institutions.

\section{The Rise of ASL as a Foreign Language}

Colleges in the USA started offering ASL courses as early as 1965 (Shroper \& Holmes, 1980, as cited in Cooper, 1997) and Canadian institutions followed suit in the 1980s. A 1981 report by the Parliamentary Committee on the Disabled and Handicapped advocated for Canadian politicians to support the deaf community through actions such as establishing sign language departments in universities and colleges (Carbin, 1996). The deaf community gaining support for sign languages through a disability rights Committee is not without tension or irony, as deaf advocacy groups have a long and complex history with the disability label, as mentioned previously in this chapter and in Chapter One. In any case, by the early 1990s, several universities established the first Canadian sign language research programs, and several colleges began offering ASL courses soon after (Carbin, 1996).

When colleges and universities began accepting ASL for credit, it opened a floodgate of enthusiasm from the hearing world (Miller, 2008). The Modern Languages Association (MLA) reported ASL enrolments in the USA jumping from 1,600 in 1990 to over 60,000 by 2002 (Goldberg, Looney, \& Lusin, 2015), and nearly doubled to 110,000 by 2013 (Looney \& Lusin, 2018). These statistics are somewhat inflated due to changes in how the MLA collected their data (Looney \& Lusin, 2018); still, ASL most recently claimed the third most enrolments behind French and Spanish. For context, French enrolments are around 175,000 and Spanish over 700,000 (Looney \& Lusin, 2018). In Canada, at least 30 colleges and universities now offer ASL, from a single course to four- 
year programs (described in the following section). ASL is also taught through community programs, private tutors, online courses, and workplace training, though data on these contexts is difficult to obtain.

The interest in ASL as a foreign language has been linked to the ongoing mainstreaming of deaf children in elementary and secondary schools (Rosen, 2014). Through contact with deaf signing peers and educational interpreters, hearing students were increasingly exposed to sign languages and - presumably to bridge the communication gap and encourage integration — began learning sign language, which may have prompted the addition of ASL as a credit foreign language in secondary school (Rosen, 2014). This interest may have given momentum to post-secondary ASL instruction.

There was also significant vocational impetus to learn ASL, as sign language interpreters have long been in short supply (Canadian Hearing Society (CHS), 2014). The disability rights movement of the 1980s, including the International Year of the Disabled in 1981, and the passing of Americans with Disabilities Act (ADA) in the USA in 1990 may have helped in recognizing the value and legitimacy of sign language interpretation (Miller, 2008; Rosen, 2014). This advance may have made deaf-related careers such as interpretation more appealing and viable (McDermid, 2010). I should clarify that ASLEnglish interpretation programs in Canada are usually four-year programs focused on interpretation, where proficiency in ASL is required at the outset of the program. Some interpretation programs also offer one- or two-year preparatory ASL programs for nonsigning prospective interpreter students. 
In Canada, the first post-secondary interpreter education programs were established in the early 1980s (Carbin, 1996). Previously, interpreter training was largely informal and community-driven, where hearing relatives of deaf people would volunteer to interpret without any formal training (Carbin, 1996). In the mid-1970s, however, changes in Canadian deaf educational policy created more demand for interpreters in schools and led fairly rapidly to the establishment of short-term (three to four weeks) and, soon after, longer-term (up to a year) formal certificate programs in several Canadian colleges (Malcolm \& Howard, 2009). By 2004, ASL was also offered in departments such as audiology, speech pathology, and special education for professionals working with deaf people, as well as for general interest in language and linguistics departments (Cooper et al., 2011; Miller, 2008). Studies too numerous to review here have explored adult learners' motivations, attitudes, and linguistic and cultural outcomes of learning ASL (for example, Beal, 2020; Brightman, 2013; Calton, 2013; Kannapell, 1989; Kemp, 1998; Krausnecker, 2015; Lang, Foster, Gustina, \& Mowl, 1996; Peterson, 1999; Prinzi, 2007).

The rise of ASL as a foreign language for hearing adults has not been not without tensions and trade-offs. In the section below I explore some of the recurrent issues to further situate the present dissertation study.

\section{Ongoing Debates About Teaching ASL to Hearing Adults}

The centralization and formal expansion of sign language interpreter education, and in particular the addition of ASL preparatory courses, meant that interpretation was no longer under the direct purview of local deaf communities. For better or worse, wouldbe interpreters soon needed little to no previous contact with ASL or deaf communities 
before beginning their studies (Beal-Alvarez \& Scheetz, 2015; Beal, Scheetz, Trussel, McAllister, \& Listman, 2018). This shift opened the doors to ASL to a much wider-and hearing - audience. The recently reported 9:1 ratio (it was 11:1 in 2009) of beginnerlevel to advanced-level courses (Looney \& Lusin, 2018) might suggest that many learners are not progressing to the point of becoming interpreters. For context, the MLA (Looney \& Lusin, 2019) reported that in 2016, Italian had a higher ratio at 10:1 and Arabic, Modern Hebrew, and Latin had a ratio of 7:1 ratio, while the other eleven foreign languages (including "Other Languages") had ratios of 3:1, 4:1, and 5:1. In any case, these statistics about ASL enrolments raises questions about the extent that ASL as a foreign language "gives back" to the wider deaf and sign language community.

Meanwhile, improved awareness and attitudes towards sign language and deaf people have been observed as a positive outcome of ASL's popularity (Brightman, 2013), but at the same time, Brueggemann (2008) observed that ASL is slowly being taken out of deaf control and serving as a "cash cow" for North American universities and their hearing patrons. Cohen Efron (2014) cited Brueggemann in her vlog post, asserting unease about the access and control hearing people have over ASL while deaf people are in a constant struggle for access to any sign language education at all. Efron echoed Eichmann (2009) and more recently Adam (2015) who both pointed to the unethical domination of hearing voices in concerns relating to sign language standardization and regulation. In Canada, Goertzen (2018) found that a number of deaf schools have closed in recent decades, leaving only eight across the country—yet ASL continues to be offered in colleges and universities to hearing adults. 
In short, perspectives on the value and impact of ASL teaching to hearing adult learners vary. One of the ways ASL teachers have tried to maintain a balance between advancing and growing ASL teaching without losing sight of core deaf principles and issues has been through the establishment of sign language teachers' associations, which, at least in Canada, are largely organized under the banner of deaf cultural institutes. I describe these organizations in the following section.

\section{The Establishment of ASL Teachers' Associations}

As ASL gained momentum in the mainstream hearing world in the 1980s, ASL teachers increasingly began to organize, resulting in local and national teachers' associations (e.g., ASLTA, SLIC). Consistently, these organizations' main priority has been standardization — of instructional practices, of curriculum and assessment, and of teacher knowledge, skills, and qualifications. Gary Olsen, former Executive Director of the National Association of the Deaf, cautioned: "Without proper standardization of curriculum, instructor qualifications, and certification, this sudden spontaneity of acceptance of ASL can be devastating. ASL, as we know it today, could be drastically altered or lost" (1988, p. 100). Olsen's concerns seem to have carried to today; recently, Canada's national sign language teachers' association, the Sign Language Instructors of Canada (SLIC; under the umbrella of the Sign Language Institute of Canada, also SLIC) expressed:

Most instructors of ASL have been, and in many cases still are, teaching signs at their own pace, in their own format, without supervision. They have never been sure which ASL textbooks to use, how to screen students, what levels to teach, how to evaluate, where to get materials, etc. Are they teaching ASL or basic 
signs? If an instructor just shows signs from a book, he/she is not teaching ASL but vocabulary. Students who have been taught in this manner have continually failed to meet the expectations of the Deaf Community for good signers. They end up knowing vocabulary but are unable to utilize the most important linguistic features of ASL. Today institutions like community colleges and other agencies are looking for qualified ASL instructors and they have brought their concerns to the attention of the Canadian Cultural Society of the Deaf. (CCSD, 2017) The SLIC in Canada, as well as the American Sign Language Teachers' Association (ASLTA) in the USA, were established with the goal of maintaining high-quality sign language instruction. More importantly for the purposes of this dissertation, these associations, as well as local teacher groups, have been an important source of information, networking, and professional development programming for ASL teachers. I introduce the SLIC and the ASLTA below.

\section{Sign Language Instructors of Canada (SLIC)}

Originally the Sign Language Instructor Certificate Organization and then the Sign Language Instructors Certification, the Sign Language Instructors of Canada (SLIC) was founded in 1981 with several goals:

To conduct a survey of sign language instructors in Canada; to investigate various sign language dialects and provide standard terminology; to develop criteria for evaluation of sign language instructors; to provide sign language instructor certificates; and to provide in-service training for sign language teachers. (Carbin, 1996, p. 325) 
Currently, the SLIC's mission is to "help ASL instructors across the nation become familiar with standard teaching methods and materials, and will provide a networking system" (CCSD, n.d.).

The SLIC is housed within the Canadian Cultural Society of the Deaf (CCSD), a not-for-profit organization, which has as its goals:

The priority of the Canadian Cultural Society of the Deaf has always been to preserve Sign Language of the Deaf Community, increase literacy levels across Canada, improve accessibility to quality education and services, and celebrate Deaf Life. (CCSD, n.d.)

In my conversations with SLIC representatives over these past several years, it appears the organization is gradually moving out from under the CCSD to become an independent affiliate. As I learned throughout this dissertation, it is very common for deaf cultural organizations to be responsible for both general deaf culture and sign language teacher professional development activities.

The SLIC is responsible for the American Sign Language Instructors of Canada Evaluation (ASLICE) program and teaching-related workshops, often offered as part of deaf culture-related conferences and events (CCSD, n.d.). The ASLICE is an evaluation and certification program for teachers with prior experience (over 400 hours within a three-year period). The application process involves first submitting a package that includes a list of prior ASL (or related) workshop attendance and classroom teaching experience; an ASLPI of 4 or 5 (i.e., native or near-native proficiency); and a reference letter from a provincial deaf cultural organization attesting to the applicant's involvement and good standing with the community (CCSD, n.d.). Following that, the candidate 
completes a three-part test about ASL structure, comprehension of a series of materials (readings, videos) provided five months prior, and a face-to-face interview to assess signing skills.

Doré (2015) found that a handful teachers in Canada are ASLICE-certified, though it was unclear exactly how many or what proportion of certificate-holders were represented. In any case, the ASLICE recognizes relevant professional development activities offered by the SLIC or elsewhere, such as by the Canadian Hearing Society (e.g., Signing Naturally training), local and provincial deaf cultural organizations, and the ASLTA, described below.

\section{American Sign Language Teachers Association (ASLTA)}

The SLIC's USA counterpart is the American Sign Language Teachers' Association, or the ASLTA. The ASLTA was established in the mid-1970s as the Sign Instructors Guidance Network (SIGN), changing the name to ASLTA in 1991 and becoming an independent organization in 2004 (Ashton, Cagle, Forestal, Greer, Jacobowitz, \& Newell, 2020). Unlike the SLIC, the ASLTA is independent of any deaf cultural organization.

The ASLTA has as its mission: "[to] provide professional development, evaluation, certification and networking for teachers of American Sign Language and Deaf Studies and to serve as a clearinghouse for information regarding the teaching of ASL and Deaf Studies" (Newell \& Cagle, 1997/2008/2013). It offers a wide range of services and resources for its members, the majority of which are publicly available via their website. The elected board consists of standard positions (President, Vice-President, 
etc.) and briefly included a number of tailored positions, including a Heritage Language Coordinator.

The ASLTA offers a two-tier teacher evaluation and certification program (formerly a three-tier program based on studies by Newell (1995abc) whose work is described in a later section of this chapter). Application for the first tier ("Certified") is for members who meet the following criteria: an ASLPI score of at least 3 (advanced proficiency); a Bachelor's degree or 20+ years' experience teaching ASL; and a minimum of three credit hours (each) in ASL literature, ASL linguistics, and deaf culture (ASLTA, 2020). Second tier (“Master”) applicants have additional requirements: an ASLPI of at least 4 (native or near-native), a Bachelor's degree, at least 1,080 hours of ASL classroom experience, including advanced courses; a range of coursework, including in curriculum and assessment; and recognized contributions to the field, including publications and conference participation (ASLTA, 2020). Employers can verify a teacher's certification status with the ASLTA by consulting a members directory (ASLTA, 2020).

The ASLTA has also done significant research and development of teaching standards, most notably the Standards for Learning American Sign Language (Ashton, Cagle, Kurz, Newell, Peterson, \& Zinza, 2012). The Standards, intended for teaching K16 (i.e., K-12 and higher education), were developed with the American Council of the Teaching of Foreign Languages and the National Consortium of Interpreter Education Centers to conform with the World-Readiness Standards for Learning Languages "5 C's" framework: Communication, Cultures, Connections, Comparisons, and Communities (Ashton et al., 2012). The document was intended not as a stand-alone curriculum, but to 
guide instructors by providing benchmark indicators of various levels of performance (Ashton et al., 2012). Five years after its release, Swaney and Smith (2017) found that the standards had not yet been widely adopted, although the authors strongly endorsed them.

Beyond the SLIC and the ASLTA, many local and regional deaf cultural associations across Canada also have the mandate to support ASL teaching. For example, the Ontario Cultural Society for the Deaf developed an ASL parent-child Mother Goose program, an ASL literacy program for hearing parents of deaf child, and provides a teacher training program and certificate for teachers using the program.

In closing, there has been—and continues to be —significant organization of ASL teachers at different levels and with varying degrees of impact. Over the decades, several common classroom practices have emerged, some of which I describe below.

\section{Common ASL Classroom Practices}

In this dissertation, I did not closely attend to ASL teachers' classroom practices, but it is nonetheless worthwhile providing some background about them in order to help the reader contextualize this study's ASL teacher-participants' later stories of classroom experience. Like the history of ASL teaching presented above, the following classroom practices were also integrated into the coding scheme for teacher-participants' interview data as a way of locating their stories of practice within previously established knowledge and accounts. Three common practices are described below: (1) the use of the textbook series Signing Naturally, (2) the value of (deaf) cultural learning outcomes, and (3) the no-voice policy. 


\section{Signing Naturally}

To begin, Mertzani (2015) described the array of sign language pedagogies that emerged in the early days of ASL instruction:

The teaching methods, especially their principles that have been tried out throughout the 1970s, cannot be related to the results of the sign linguistic analysis of that time. They are, instead, beliefs that underlie and support some techniques of analysis, but they are not the results or conclusions of sign linguistics analysis. They simply preceded sign linguistics. This is the reason why, in one single decade, different programmes attempted to employ four methods, with different principles and techniques. (p. 51-52)

With the publication of the Signing Naturally textbook series, though, some consistency was found. Rosen (2010) and more recently Swaney and Smith (2017) found that Signing Naturally was the most commonly used textbook across the USA. Doré (2015) identified many mentions of Signing Naturally being used in classrooms as well as the basis for teacher training programs across Canada. Harding and MacFayden (2014) found that use of the Signing Naturally series was one of the few commonalities in ASL programs across British Columbia. The ASLTA's annual conference also often includes workshops based on the Signing Naturally series.

Signing Naturally was developed by three deaf ASL teachers in the USA based on the functional-notional teaching method. In brief, the functional-notional method is a communicative method (which assumes the purpose of language is to communicate with others) that grew out of discussions about adult foreign language learners' needs in the 1960s and 1970s, where it was decided that "the ability to use real, appropriate language 
to communicate and interact with others is - and should be - the primary goal of most foreign language learning" (Finocchiaro \& Brumfit, 1983, p. 10). The method breaks down language into units that students can master one by one, where each unit is centred on a language situation, such as, in the case of Signing Naturally, finances, describing objects, and talking about the weekend.

The series includes three books organized into 25 units and three levels: Level One includes units 1-6 and 7-12, Level Two includes units 13-17, and Level Three includes units 18-25. The Signing Naturally publisher's website includes additional material, including a five-point evaluation sheet (rubric) for each unit and slide decks to support additional discussion. A brief scan found a plethora of Signing Naturally videos and discussion groups in public forums such as YouTube and Facebook.

Smith (1988), one of the three (deaf) authors of the ever-popular Signing Naturally textbooks, explained that the series was designed with "detailed lesson plans that included what to teach and how to teach it ... [and] activities and materials (handouts, worksheets, transparencies, and videotapes) to help teachers successfully implement the curriculum" (Smith, 1988, p. 179). She and her co-authors recognized that most ASL teachers are not trained specifically as language teachers. ASL instruction is still in its early stages and there are few resources for the teacher to turn to. Therefore, we [the authors of the Signing Naturally series] wrote detailed lesson plans that included what to teach and how to teach it. We developed activities and materials (handouts, worksheets, transparencies, and videotapes) to help teachers successfully implement the curriculum. (Smith, 1988, p. 179) 
In other words, the textbooks were expressly designed to provide some form of standardized practice for inexperienced teachers by providing everything that might be required in a classroom, from content to delivery—ready-made lesson plans, homework, assessments, classroom management guidance, and so on.

The timing of the series' publication was notable. By the late 1980s, ASL was in the midst of its unexpected "boom", which provoked an ongoing and increasingly pressing shortage of qualified ASL teachers that led to ad hoc hiring. Smith (1988) explained that their series was in response to the issue:

ASL programs often select teachers for their language fluency rather than their background in language teaching. These teachers, in turn, base their classes on student textbooks or try to develop their own instructional materials. There is no overall curricular idea which can help teachers (1) establish a cultural context for language instruction, (2) make decisions about how to sequence course materials, and (3) develop activities which allow students to progress from one-word responses to spontaneous expression of thoughts and feelings on a discourse level. (p. 171)

The authors received USD $\$ 90,000 /$ year for three years from the US Department of Education to develop the book, which now has several revised and expanded editions. Signing Naturally is by far the most popular ASL curriculum series used today (Calton, 2013; Goertzen, 2018; Rosen, 2010; Swaney \& Smith, 2017) and thus a uniting force in an otherwise diverse profession. One of the possible reasons the series has gained such widespread popularity is that, as described above, it is "ready-to-use". It was designed to include all of the teaching and assessment resources, tips for practice, and 
extra resources that a teacher, new or experienced, might need in the classroom (Smith, 1988). Because many ASL teachers were arriving to teaching with no background, Signing Naturally was a way of promoting standard methods outside of formal training mechanisms.

Another suggested reason for its popularity is that deaf teachers want to support deaf authors (the authors of Signing Naturally are deaf) by using deaf-authored materials (Thoryk, 2010). Thoryk (2010) and others (e.g., Quinto-Pozos, 2011; Rosen, 2010) have expressed hesitation not about Signing Naturally specifically, but about the uncritical application of ASL teaching materials that have not been evaluated empirically.

In terms of content, Signing Naturally does not overtly include lessons on deaf culture - at least not in the student workbooks. In the teacher workbooks there are cues and suggestions for aspects of deaf culture to highlight in each lesson. The related learning outcome of developing an awareness and respect for deaf cultural norms are discussed in the next section.

\section{Cultural outcomes}

As indicated by Donna in the opening of this dissertation, an important learning outcome of ASL instruction is developing learners' awareness of sign languages and deaf culture. Broadly speaking, the idea of language courses fostering learners' cultural awareness for greater social equity is not new and is a common outcome of spoken language classrooms. Gagné and Gordon (2015), for example, suggested that teaching immigrant students with a social justice framework can improve their language learning and cultural integration. Likewise, Gagné and Gordon (2011) followed two groups of high school students — one English language learners and the other Canadian-born native 
English speakers - as they developed mutual awareness and respect through a variety of language learning activities, including collaborative narratives. Other studies have shared similar promising findings (e.g., Dagenais, Walsh, Armand, \& Maraillet, 2008; Forsman, 2020).

For ASL learning specifically, several studies have examined the impact of ASL learning on hearing learners' attitudes and orientations toward sign languages and deaf people, most with promising results (e.g., Beal, 2020; Brightman, 2013; Calton, 2013; Kannapell, 1989; Kemp, 1998; Krausnecker, 2015; Lang, Foster, Gustina, \& Mowl, 1996; Peterson, 1999; Prinzi, 2007). In terms of teaching practices, Calton (2013) found some differences in how spoken language teachers accomplished their goals compared to ASL teachers:

ASL teachers place less emphasis on language proficiency and feel tasked to prove to their students that ASL is a genuine language... ASL teachers expressed a specific desire that ASL students leave their class with respect for Deaf people and ASL. (p. 79)

Similarly, in Trowler and Turner's (2002) study of deaf academics' induction in universities, one participant stated,

I want the students to achieve their full Deaf awareness. I want them to have a full understanding of the language, of the community and the culture and I want them to have the ability to communicate well with deaf people in a natural way.... They will know that there are different groups and that there is more to deafness than just a hearing loss. They will know about language, community and culture. (p. 248) 
Thus, ASL classrooms are sites for social intervention for increased deaf awareness, dispelling erroneous assumptions, and reversing discriminatory behaviours related to the nature of sign languages and deaf people.

One of the ASL teachers who participated in the present study, David, was explicit about his goals to raise his students' awareness of their hearing privilege. In broad terms, privilege can be understood as "the other side of oppression" (Ferguson, 2014). In deaf studies, many scholars have reflected on hearing privilege. Eckert and Rowley (2013) mentioned audiocentric privilege in relationship to audism, or the privilege of not experiencing discrimination based on hearing. Lane et al. (1996) talked of codas (i.e., children of deaf adults) not being fully accepted members of the deaf community despite having a deaf sense of self because they still have the lifelong privilege as hearing people of being able to return to the hearing world at any time when not hearing may cause inconvenience or distress. Komesaroff and McLean (2006) focused on able-bodied privilege as it related to oppressive (ableist) deaf education practices privileging hearing children and dismissing deaf children as lesser and needing "fixing".

Tuccoli (2009) defined hearing privilege as advantages or entitlements that are enjoyed by people who can hear which are denied to those who are Deaf. These advantages give hearing people power and authority to decide how society should be designed (p. 23, as cited in Aubrecht and Furda, 2012). 
Building on Tuccoli's research, deaf-hearing team Aubrecht and Furda (2012) listed specific hearing privileges to illustrate how privilege can manifest itself in specific and abstract ways:

Hearing privilege is not being a "new experience" for others.

Hearing privilege is having people assume you are a capable parent.

Hearing privilege is people assuming you can drive, ride a bike, operate machinery, be a pilot ... having people assume you can do almost anything for a career.

Cox (2010) did the same, focusing on hearing privilege that creates exclusionary, paternalistic, and dismissive deaf-hearing relationships (e.g., being told "It wasn't funny anyway" about missed jokes by hearing friends and relatives). Santini (2011) adapted McIntosh's (1990) White Privilege Invisible Knapsack classroom activity (designed to encourage students' reflection on white privilege) to suit reflections on hearing privilege. In practical terms, ASL students often learn deaf "etiquette" both in class as well as through participation in extra-curricular deaf club activities, though their presence at the latter is somewhat contested. Common in-class practices to develop students' awareness include activities to flag norms of respect when in the company of a deaf person (e.g., adhering to a no-voice policy in class, described below) and fostering a positive attitude about sign language and deaf people. Schornstein (2005) considered the latter to be the most valuable transferable skill for a hearing signer: "We [deaf people] can occasionally accept someone's limited skills if the person has the right attitude. It is important to foster a positive outlook in our students because a bad attitude can be a 
deterrent to effective interpretation" (p. 411). The next section explains the no-voice policy in more depth.

\section{No-voice policy}

One of the most pervasive practices in ASL classrooms is the no-voice policy (also voice-off). Cooper et al. (2011) found that of the 301 institutions they surveyed in 1994 and 180 in 2004, roughly $70 \%$ and $80 \%$ respectively had an explicit or implicit policy (i.e., not stated but voice discouraged) about the use of voice in the ASL classroom. Over $80 \%$ of both teachers and students in both samples declared using voice little or not at all (Cooper et al., 2011). Cooper and her co-authors hypothesized that novoice policies are put in place not only to support cultural learning aims (respect for deaf teachers, deaf enculturation, etc.), but also to "establish ASL as a language independent of English and to disallow the use of English and maintain the integrity of each language" (p. 321). As described earlier in this chapter, a common misconception is that ASL is a manual derivative of English rather than a distinct natural language. Emphasizing the distinctness of ASL and English while demonstrating their relationship (e.g., finger spelling) are common classroom practices (Schornstein, 2005).

Having described some of the common ASL classroom practices, in the next section, I summarize the current presence of ASL in Canadian post-secondary institutions. I must note that although ASL is taught to hearing adult learners in a number of contexts, including in community-based continuing education programs (e.g., ASL classes offered by the CHS), private lessons, and online asynchronous programs, these contexts are difficult to trace and examine, as they are not centrally coordinated. They are also distinct professional settings from colleges and universities, where in the latter 
teachers are arguably more likely to face more rigid curriculum requirements, especially in programs that provide certification and/or accreditation (i.e., pre-/interpreter training), as well as accountability and oversight if a degree or credit is being granted to learners who complete the course/program. In this dissertation, I primarily focus on ASL courses taught in colleges and universities. With the exception of Michelle, all of the teachers in this study spent the majority of their careers in either or both college and university settings.

\section{ASL in Canadian Colleges and Universities}

In Canada there are currently (as of 2019) over 30 post-secondary institutions where ASL is taught. Appendix A summarized the institutions that offer ASL, the department or faculty where the course/s and/or program is housed (e.g., Continuing Education, modern languages department), whether there are credentials associated (e.g., Certificate in ASL), and the level/s and number of courses in ASL and deaf culture that are offered. This data was collected through a thorough manual scan of all of Canada's colleges and universities' websites. Because the data was drawn from publicly available information rather than through, for example, an in-depth national questionnaire of program administrators. Consequently, some courses or programs may have been overlooked or misrepresented if, for example, the website was incomplete or out-of-date. This summary did not include institutions that accepted ASL for credit but did not offer

it. Likewise, I did not attempt to survey ASL programs offered outside of post-secondary institutions, such as community courses with, example, the Canadian Hearing Society (CHS) or deaf cultural organizations or private lessons. There programs are difficult to track, as they are often demand-driven. 
To sum up the data presented in Appendix A, there are 15 universities and 18 colleges across Canada that offer ASL courses. The greatest concentration is by far in Ontario (eight universities and 11 colleges), which is to be expected given the higher concentration of post-secondary institutions (Ontario is also home to the Deaf Culture Centre in Toronto and the Canadian Association of the Deaf (CAD) headquarters in Ottawa). Saskatchewan had no listed post-secondary ASL offerings at the time, which perhaps reflected the lower vocational demand given the province's policies that were not favourable to manual deaf education and sign language services (Saskatchewan Human Rights Commission, 2016). Manitoba had two programs, one college and one university, that collaborated to offer a joint BA-Diploma program. This joint program was noteworthy because interpretation programs usually lead to a college diploma, not bachelor's degree (Malcolm \& Howard, 2009). Nova Scotia likewise had only one college offering ASL as part of an interpretation program. Quebec had no post-secondary ASL programs, although it did have LSQ courses and programs (not listed).

There is not a standard naming system for ASL course levels (e.g., ASL 101 vs. ASL I), which made it difficult to determine which levels were offered in each institution. A scan of the data suggested that many institutions offered levels beyond beginner-level. Likewise, although only a handful of the programs summarized had explicitly labeled deaf studies courses (culture, history), most likely all ASL courses include some form of teaching on the subject, as described in the previous section.

In line with previous literature (e.g., Gray, 2018), I found that ASL was housed in a range of departments and faculties. Nearly half were in continuing education as standalone courses and four were in programs related to special education, child services, or 
health. Eleven were in departments of linguistics, applied linguistics, or modern languages and two institutions had ASL and deaf studies departments. Roughly half of programs offered a certificate, diploma, or other form of recognition for ASL course completion (i.e., above and beyond individual course credit).

This data alone indicated little about the general status of ASL instruction in Canada or about the professional lives of its teachers whose experiences surely varied tremendously. Thus, the data in Appendix A and described above was merely a backdrop for the stories of the teachers who were the focus of this dissertation.

In this chapter, I have provided the reader with a selective review of the history and status of ASL in Canada, how ASL gained widespread popularity as a foreign language among hearing adult learners and some of the tensions that arose as a result of its growth. I also described some of the collective efforts at developing the profession, including ASL teachers' associations and common ASL classroom practices. As was previously noted in Chapter One and as is described further in Chapter Three, in the present study I considered teacher-participants' stories to be intertwined with the social and historical context of ASL and ASL teaching as well as informed by established (e.g., second language acquisition theory) and embodied (e.g., personal practical knowledge (Connelly \& Clandinin, 1990)) theories of practice. The literature in this chapter thus informed my conceptualization of the patterns that appeared in the teachers' stories about their practice, which are told in Chapters Six to Eight and situated theoretically in Chapter Nine. 
Next, in Chapter Three, I turn my attention to the key scholarship that guided the present dissertation, including theoretical literature about narrative inquiry in relation to teachers' practices and empirical literature about ASL teaching as a profession. 


\section{Chapter Three: Theoretical Tapestry and Literature Review}

In Chapter Two, I provided context for the present study by situating it within the sociohistorical context of teaching ASL to hearing adults, including some of the debates and common practices that define it. In this chapter, Chapter Three, I outline the "tapestry" (Hollingsworth, Dybdahl, \& Minarik, 1993) of narrative and learning theory and the empirical scholarship about ASL teaching as a profession that together informed this research. In the first part of the chapter, I begin with a brief section below about the history of the "narrative turn" in qualitative research that coincided with shifting conceptions of teacher knowledge in general education and thus opened the door to new forms of research about teachers' professional lives. I then outline four principles of narrative and the key narrative scholarship that informed the present study. In the second part of the chapter, I review a selection of findings from empirical research about ASL teachers that give additional insight into the profession, including the findings from a study of Canadian ASL teachers that I conducted early in my doctoral program (Doré, 2015).

\section{The Teacher-Narrative Turn}

In the first half of the $20^{\text {th }}$ century, it was considered largely unnecessary for teachers to have extensive theoretical or "esoteric" knowledge such as that gained through higher education and ongoing in-service training (Hargreaves, 2000). When standardized tests were introduced in the 1930 s as a way to efficiently "process" learners in "large batches" as in a factory (Hargreaves, 2000), teachers were cast as transmitters of knowledge or "technicians" completing defined tasks that did not require an extensive knowledge base (Connell, 2009). Clandinin and Connelly (2000) argued that the trend of 
devaluing teachers' lived experiences or "professional memory" (p. 36) as a source of knowledge continues in the $21^{\text {st }}$ century and forms what they refer to as the grand narrative (defined in the following section) of contemporary education. Early teacher education was based on an established "grammar of teaching", passed along between generations of teachers (Hargreaves, 2000), and highly gendered, where elementary school teaching was primarily considered women's work (i.e., maternal care) and high school and post-secondary was men's work (i.e., moral and intellectual development; Connell, 2009).

The many social movements of the mid- $20^{\text {th }}$ century that pushed for social equality, including gender equality, and the postwar surge in demand for education contributed to an increase in universities and colleges, where teacher education was gradually established (Connell, 2009). The university setting fostered the growth of humanistic theories of teaching and learning throughout the 1960s and 1970s, such as theories of teaching as a craft, teacher knowledge as embodied, and teachers as critical intellectuals (inspired by theorists such as Paolo Freire). These theories ran counter to the dominant view in education at the time, technical rationalism, that held that knowledge was objective, existed outside of the body and the social world, and that it could be transmitted from one person, a teacher, to another, a student (Johnson, 1987, as cited in Clandinin \& Connelly, 2000).

By the 1980s it was more widely understood that teachers' practice was not solely shaped by their education or formal training; rather, practice was an ongoing process where feelings, values, and beliefs combined with experiential and theoretical knowledge (Elbaz, 1981). Elbaz (1981) developed a framework of teacher practical knowledge that 
included the interrelated categories of teachers' knowledge—subject matter, curriculum, instruction, self, and milieu of teaching. Lampert's (1985) personal knowledge extended Elbaz' category of knowledge of the self and claimed that teachers' practice is guided by knowledge of the self as well as of the child and of the interpersonal relationship between teacher and child. Drawing on both of these, Connelly and Clandinin (1988) coined the term personal practical knowledge, which is, in brief, a "teacher's knowing of the classroom" (p. 25) that is "shaped by the personalities of the teachers themselves, their past experiences, and how they view teaching" (Calderhead, 1996, p. 718). Its creation is deeply contextual, applied according to the "exigencies of a present situation" (Connelly \& Clandinin, 1988, p. 25). Personal practical knowledge was a key theoretical lens in this dissertation and is discussed again later in this chapter.

The development of the above humanistic theories in teacher education coincided with the "narrative turn" in qualitative research that included scholars such as Ricoeur (1984-1988), Bruner (1986, 1987, 1991), and Polkinghorne (1988) who built on the broader narrative turn in 1960s literature (Hyvärinen, 2016) and advances in feminist narrative research (Lyons, 2007). As Polkinghorne (2007) expressed:

Stories are ubiquitous, appearing as historical accounts, as fictional novels, as fairy tales, as autobiographies, and other genres. Stories are also told by people about themselves and about others as part of their everyday conversations. In addition to the stories that appear in people's ordinary conversations, narrative researchers study stories they solicit from others... (p. 471)

The development of narrative as an approach to empirical study meant that, as the present study does, teacher's professional lives, including their knowledge and learning, could be 
studied through their stories of practice. The principles of narrative that guided the present study are described further in the following section.

\section{Principles of Narrative}

My priority in this dissertation was to speak to teachers' professional life stories holistically and honour the individuality of their "professional memories" (Clandinin \& Connelly, 2000, p. 36), i.e., their accounts of lived experience, so that ASL teachers who might read this work seeking to relate to their colleagues would find whole, not fragmented, stories and storytellers. As I explain later in this chapter, there are few academic publications about ASL teachers and their practices, and fewer still that have applied a narrative lens. Yet, in ASL and deaf studies, narrative has a long history of being the object of study by both sign language narrative researchers and discourse analysts (Mulrooney, 2009; Roy, 2011; Winston, 1999). The social practice of storytelling is firmly entrenched in deaf culture, where storytelling is an important way of passing along cultural and social information, both horizontally among peers and vertically through generations (Lane, 2005). Corker (1996) highlighted the social function of stories:

This [the importance of storytelling in Deaf culture] is partially because Deaf people may be cut off from full access to formal, mainstream information channels, and a lot of social activities are taken up with the exchange of information about people and events in their community. The main channels of communication are through rumour or general hearsay, and gossip. (p. 15)

Narrative approaches have been applied to explore topics like personal and collective deaf identities and histories (Brueggemann, 2007; Corker, 1996; Cyrus, Katz, Parson, \& 
Hole, 2004; Kelly, 2001; Schornstein, 2005; Snoddon, 2014; Winston, 1999). They have not been often used to explore the professional lives of ASL teachers, hence the focus of this dissertation.

The research approach that best suited my research aims was narrative inquiry. Unlike other qualitative methodologies that use stories as data to be deconstructed in order to arrive at themes, narrative inquirers can collect a variety of data —including field notes, journal entries, conversational interviews, letter writing, written accounts, and autobiographical writing (Savin-Badin \& Van Niekerk, 2007)—with the ultimate outcome of presenting whole stories in response to research questions (Polkinghorne, 1995). At its core, narrative inquiry is driven by assumptions about the nature of storytelling as socially enabled and constrained social action. Chase (2005) outlined four principles of narrative, each described below, that reflect the narrative theoretical framework underpinning the current study's methodology.

\section{Principle One: Narrative is a social action.}

People do things with stories (Gubrium \& Holstein, 1999), including make sense of experiences and create and assert their identity in social circles (Clandinin, 2007). By nature, language reveal something of widely held beliefs, assumptions, and practices or grand narratives (defined below in Principle Two). As such, stories are edited throughout and between tellings to different people in different situations, each time performing their own social functions (Gubrium \& Holstein, 1999). A social function addressed in the present study was how for teachers "storytelling can be a means of laying claim to a particular notion of professionalism" (Savin-Badin \& Van Niekerk, 2007, p. 463), or how 
their stories responded to calls to membership or nonalignment with broader ideas or grand narratives of ASL teaching.

\section{Principle Two: Narrative is socially enabled and constrained.}

Narratives are not apolitical, unemotional, and without ascribed value; rather, stories, from their content and organization to their delivery and re-telling, are "enabled and constrained by a range of social resources and circumstances" (Chase, 2005, p. 657). Philosopher Lyotard's (1984) concept of grand narratives argued that "language was performative and that people engaged in pragmatic language games that legitimated meta-narratives, the existing power relations and denied heterogeneity" (as cited in McDermid, 2009, p. 228). Grand narratives drive what Clandinin and Connelly (1996) referred to as the sacred stories of teaching, the morally and ethically laden stories of "good teaching", that can push teachers to tell other types of stories such as cover stories that both justify and repent for non-alignment with a sacred story, and secret stories that confess to non-alignment with a sacred story.

\section{Principle Three: Narrative is performance.}

Chase (2005) defined narrative as "socially situated interactive performances", where "[a]mong other things, narrators explain, entertain, inform, defend, complain, and confirm or challenge the status quo" (p. 657). Moreover, narratives are transient, ephemeral:

As much as the storyteller can be the author of his or her narrative, he or she is also an editor who constantly monitors, manages, modifies, and revises the emergent story. Invoking shifts in footing-such as referring to the position from which an account is offered- is one prominent type of "narrative editing" through 
which storytellers attend to the perspectives of personal stories and to the ways they will be heard. Editing confirms that storytellers are never narratively "frozen" as authors of the texts they produce. (Gubrium \& Holstein, 1998, p. 170) The ASL teachers' stories presented in this dissertation were told in a here and now that has gone and since morphed into something else by the point of this publication.

\section{Principle Four: Narrative researchers are co-narrators.}

Narrative studies are often interview-based, where the interviewer is an active interlocutor in the process of storytelling, not a passive bystander or recipient of an unemotional or value-free chronology (Gubrium \& Holstein, 1998). Rogers (2007) explained:

the narrator (and the narrative) cannot be separated from the social context... These researchers view the narrator as self-reflective within a social context. In interview analysis, then, it is possible to interpret relations between external (social) contingencies and internal (individual and self-reflective) experience.

Often this includes an examination not only of the participants' social experience but also of multiple truths and shifting identity positions. (p. 102)

In the present study of ASL teachers, I was external to the lived experiences that informed the stories told by the study's teacher-participants, but internal to the experience of their telling for the purposes of this study. The teacher-participants related to me in different ways at different points in their storytelling. At times, they positioned me as an insider with shared knowledge, and so some aspects of their stories could be glossed over, my understanding taken for granted. Other times their stories were told to me as an outsider to whom the story needed to be explained carefully. In the process of coding 
teacher-participants' interviews, I made note of these shifts in how they constructed me as their interlocutor, as they helped to flag other audiences and narratives (grand or otherwise) they were speaking to, whether in alignment, rejection, or otherwise.

The above narrative principles formed the theoretical basis for my narrative methodology. The procedures for data collection and analysis that I used in the study are described in Chapter Four. First, I describe below several intertwined theories that proposed that teachers' narratives hold the key to understanding lived experience as a source of professional knowledge.

\section{Knowing-in-Action and Teacher Narratives}

Building on the momentum of narrative inquiry in the 1970s and 1980s, several educational researchers adopted narrative approaches to understanding teacher knowledge and practices, which as described earlier in this chapter were not previously commonly considered in educational research. A shared influence in this early scholarship was educational philosopher John Dewey (1916) whose ideas of experiential learning from several decades earlier inspired work about teachers' learning through experience and reflection on experience. Following Dewey, Schön (1983, 1987, 1991, as cited in Clandinin \& Connelly, 2000) proposed the term reflective practitioner as a way of describing teachers' learning through practice and reflection on practice, or knowingin-action. Clandinin and Connelly (2000) spoke to the value of Schön's (1983) proposition:

Schön makes it possible for many of us to tell the story of teacher education (and other professional education) in a way that runs counter to the technical teacher 
education we are encouraged to sponsor and study, and he makes it possible for the story to legitimate our professional memory and reflective practice. (p. 36). The professional memory, "a rich whole", extends to recent practices as well as long-ago practices as well as "the remnants of practices discredited by the grand narrative [of technical rationalism]" (Clandinin \& Connelly, 2000, p. 36). In brief, technical rationalism refers to the reduction of teaching to "a formulated set of rules" (Clandinin \& Connelly, 2000, p. 36) driven by the image of the teacher as a "compliant technician" (Connell, 2009) delivering a set "grammar of teaching” (Hargreaves, 2000). According to Schön (1983), teacher narratives are a way of expanding and validating the professional memory that technical rationalism had reduced.

Olson (1995) likewise advocated for teachers' narrative authority, i.e., the legitimacy of teachers' knowledge derived through their professional practice, and that teachers' stories of experience were a window to their "contextualized knowledge" (p. 122), or their knowing-in-action. This stance is the foundation of the present thesis. In the remainder of this section, I described the work of several other teacher narrative scholars who developed further ideas about the nature and role of teachers' narratives.

Elbaz-Luwisch was among the wave of early educational scholars who conceptualized teachers' knowledge as stemming from both theory and practice (e.g., Elbaz, 1981). Elbaz-Luwisch (2005) advocated for attentiveness to teachers' stories as a way of understanding the experiences, interactions, and emotions that led to their knowledge development and informed their classroom practices. She also viewed storytelling as a "convivial practice" (Elbaz-Luwisch, 2005, p. xvii) where peopleteachers, researchers, etc. - come together to work on making and figuring out meaning. 
One of her main theoretical influences was philosopher and literary critic Bakhtin (1981) whose concept of polyphony (meaning "multiple voices") directed Elbaz to consider teachers' narratives in terms of the various voices at play in any given story (Elbaz, 2005). Most notably, in her work she unpacked the "moral voices" involved in teachers' storytelling — stories of care, loss, hope, making a difference, and so on (Elbaz, 1992, 2005).

Clandinin and Connelly (1996) developed a conceptual framework around the types of stories that emerge in narrative inquiry: teacher stories and stories of teachers (as well as the parallel school stories and stories of schools), where teacher stories are stories told by teachers to themselves and others, and stories of teachers are stories told about teachers by other teachers or parents, administrators, policy documents, academic theory and literature, and so on (Clandinin \& Connelly, 1996).

Craig $(1999,2007)$ built on Clandinin and Connelly's (1996) framework by reconceptualizing stories as paired narratives, or parallel stories (Craig, 1999), which she conceived of as existing in relation to one another as story constellations (Craig, 2007). She also added her own parallel stories: stories of community and community stories (Craig, 2000) and stories of reform and reform stories (Craig, 2001, as cited in Craig, 2007).

Looking more closely at teacher stories, Clandinin and Connelly (1996) proposed that teachers tell several different kinds of stories—sacred, secret, and cover storieswhich I mentioned briefly in the previous section and unpack further here. Sacred stories are implicit rights and wrongs, that teachers might identify as the way to be/do/know based on their interactions with their community of practice, superiors, students, and so 
on (Clandinin \& Connelly, 1996). Clandinin and Connelly (1996) refer to these "morally laden mandates" as the conduit (as cited in Craig, 1999, p. 399). Sacred stories, or more particularly violating them, can push teachers to live and tell secret stories, where experiences are modified or untold so as to maintain the face of a "good" teacher, to not disturb the sacred story (Clandinin \& Connelly, 1996). On the other hand, teachers may tell cover stories, where teachers tell about their experiences to make themselves look like experts, confident, in control, and which "enable teachers whose teacher stories are marginalized by whatever the current story of school is to continue to practice and to sustain their teacher stories" (Clandinin \& Connelly, 1996, p. 25).

Beyond their theoretical and empirical contributions, Clandinin and Connelly (2000) also made significant methodological contributions with their definition of narrative inquiry:

It is collaboration between researcher and participants, over time, in a place or series of places, and in social interaction with milieus. An inquirer enters this matrix in the midst and progresses in the same spirit, concluding the inquiry still in the midst of living and telling, reliving and retelling, the stories of the experiences that made up people's lives, both individual and social. (p. 20) They referred to the process of interpretation and reinterpretation of stories as restorying, which involves analysis of storied data according to three dimensions: sociality as personal (intrapersonal) and social (interpersonal) interaction, temporality as continuity over time across the narratives, and space as the spaces and places in the storyteller's narrative landscape (Clandinin, 2007; Clandinin \& Connelly, 2000). This three- 
dimensional framework was the principal guide for my narrative analysis in this study, as described below.

\section{Bringing it All Together}

The three dimensions encapsulated the above-described theories of knowing and learning and theories of narrative as enabled and constrained social action as well as the sociohistorical context of ASL teaching (Chapter Two) and the common practices of ASL teaching (later in this chapter). Figure 1 shows my interpretation of the relationship between all of these elements. In brief, the process of narrative analysis filtered stories of experience through the three dimensions of stories (sociality, temporality, and space) of the past, present, and future. These three dimensions of stories illuminated elements of the theoretical framework guiding this dissertation. The next paragraphs look more closely at this relationship.

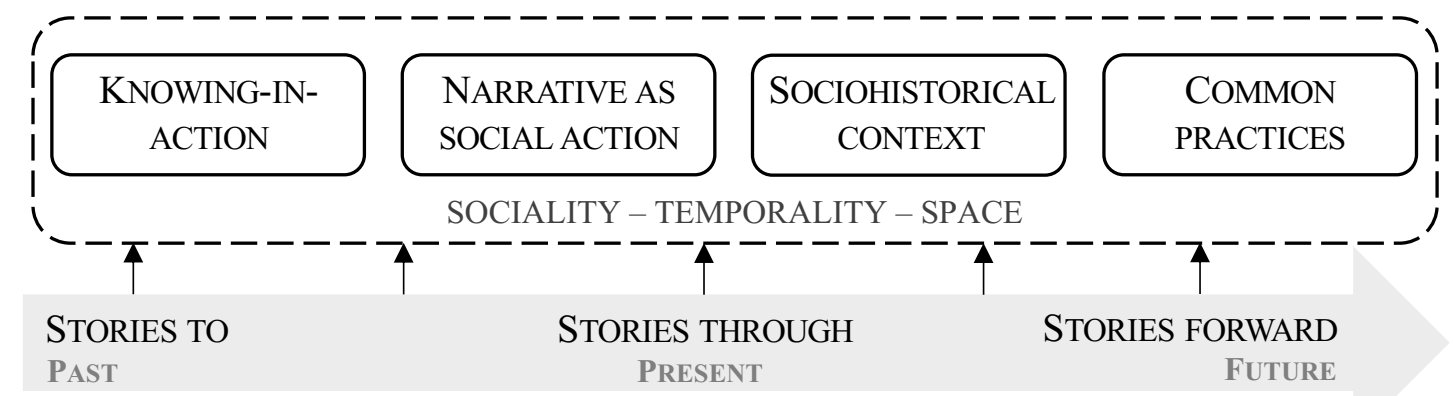

Figure 1. My interpretation of the theoretical relationship between stories, context, and literature.

Sociality encapsulated learning as per Dewey (1916) and Schön (1983). Lave (1993) also explained learning as an inherently social process (space) that happens over time (temporality): 
knowledge and learning will be found distributed throughout the complex structure of persons-acting-in-setting. They cannot be pinned down to the head of the individual or to assigned tasks or to external tools or to the environment, but lie instead in the relations among them. (p. 9)

Through the lens of sociality certain features of teacher narratives come forward, such as who was involved in the story's action, who enforced or rejected the grand narrative of the story, and, equally importantly, the relationship the storyteller established with the me, researcher and their interlocutor, as a social other.

On the macro level, temporality pointed to the chronology that was used to structure this dissertation — pathways to (past), pathways through (present), and pathways forward (future). On the micro level, stories of the past can extend to the limits of the professional memory, or, as implied by Connelly and Clandinin's (1988) personal practical knowledge, to the limits of the teacher's life memory, as their past experiences as a teacher and as a learner play a part in informing their present practice.

For the present research, Ellis' (2018) idea of language teachers' language lives, i.e., their prior learning, using, and teaching of languages, brought ASL teachers' personal practical language knowledge into the frame of narrative reference. Attending to the early stages of teachers' pathways (see Chapter Six) was thus important in order to understand their later experiences (Chapters Seven and Eight). Stories of the future, were thus simultaneously reflective, looking back, and anticipatory, looking forward, which reinforced Schön's knowing-in-action and the dialectic relationship between knowing (or theory) and practice. 
Finally, space and place, or what Lave (1993) referred to as the environment was the third dimension of stories. Stories, as described earlier in this chapter, are told at a certain time and place to a certain audience; their relationship to their context was thus essential to understanding the experiences described in stories, whether past, present, or future, as well as the experiences of their telling. In this dissertation, I have provided background information about the spaces that ASL occupies in higher education and society more broadly (Chapter Two) and provide in the sections that follow below more context about the spaces of ASL teaching as a profession.

To summarize, the above theoretical framework suggested that teachers develop knowledge and personal theories of teaching and learning through practice, and that their practice reinforces, rejects, or shifts their previous knowledge. In other words, in this dissertation I took a dialectic view of the relationship between theory and practice in which the two were not separable; theory guided experience, and experience generated theory. I also maintained that narrative inquiry is a way to access teachers' knowledge and understanding of context, as teachers' stories are multi-layered, multi-dimensional, and reveal much about the social, temporal, and spatial features of their experiences and (re)telling.

Looking forward, in the next part of this chapter I review a selection of empirical studies about ASL teachers. I begin with my own study (Doré, 2015) of ASL teachers in Canada followed by studies of teacher standards and professional development. This literature informed the present study as it flagged aspects of teachers' professional context that may have contributed to shaping their stories. 


\section{Review of Studies of ASL Teachers}

Despite its ongoing popularity as a foreign language, ASL teaching as a foreign language is a relatively new area of applied sign linguistics, which is itself a young field, and this has had an effect on ASL teaching practices. Recent publications such as the Routledge Handbook of Sign Language Pedagogy (Rosen, 2019), however, suggest that the research area is active. There have been few empirically derived publications about ASL teaching and there is currently no journal dedicated to ASL instruction (Cooper, Reisman, \& Watson, 2008).

Kiely commented on the state of ASL teaching research in his keynote address at the first Applied Sign Linguistics Symposium in 2009:

In my feeling, in teaching English as a foreign language or teaching foreign languages, when the work started forty or fifty years ago, the task of language description had been done; dictionaries were there; grammars were there; that had been going on for 100 years. It seems to me that you have the challenge at the same time of describing sign languages, and trying to negotiate issues of standards, variation etc. at the same time as working out how to teach sign language and what are the involved processes. It is a very complex issue with less activity. (as cited in Mertzani, 2015, p. 53)

In my own scan of the literature, I have likewise found that applied sign linguistics and ASL teaching scholarship is developing many branches at once, only some of which are robust enough to support in-depth empirical discussion. Studies that focus on ASL teachers as professionals and as more than a factor in a learner's instructional context are few. 
The lack of research about ASL teachers has been noted by several scholars over the decades. In the early days of the ASL "boom" in enrolments, yet nearly three decades after the birth of sign linguistics, Wilcox (1988) observed, "ASL has been slow to garner any degree of status in the academic community" (p. 101). Indeed, as mentioned in Chapter Two, ASL was not widely recognized as a "real" language even by its own users until the 1970s with advances in theoretical and social linguistics (Bellugi \& Fischer, 1972; Stokoe, 1978; Stokoe, Casterline, \& Croneberg, 1976; Woodward, 1972). By the end of the $20^{\text {th }}$ century, Peterson (1999) claimed that there was still too much research energy expended on supporting claims about the legitimacy of ASL as a language, rather than on depth and breadth. Another ten years after that, Brueggemann (2008) noted that academic acceptance of sign languages by the academy at large and by key language organizations (e.g., the MLA) is still a fight.

By the time ASL gained traction in the foreign language classroom, applied and theoretical linguistics that had already developed a strong academic foundation. On the one hand, many theories and practices around language pedagogy had already been established, and so ASL teachers and scholars could draw on these to inform their work. On the other hand, those established theories and practices were overwhelmingly based on spoken languages with a much longer history of recognition and analysis (QuintoPozos, 2011). There have been compelling arguments for developing a sign language differential approach rooted in deaf epistemologies (i.e., deaf ways of knowing) and creating frameworks around the unique features of sign languages (Vermeerbergen, 2006), and such scholarship is showing signs of growth. 
Though studies of ASL teachers are relatively few, they do exist, and they offer valuable insights that informed my dissertation. Below, I describe a recent study about Canadian ASL teachers that I conducted in the early stages of my Ph.D. studies, followed by a discussion of other empirical literature primarily related to ASL teacher standards, qualifications, and professional development. Few of the studies in my review highlighted the voices of teachers themselves; still, they provided insight into broader narratives about ASL teaching as a profession and were thus a valuable lens in my analysis of this study's teacher-participants' stories.

\section{Profile of Canadian ASL teachers}

Early in my doctoral program I conducted a study (Doré, 2015) of Canadian ASL teachers of hearing adult learners (CUREB clearance \#102303) in order to establish the groundwork for my dissertation and build credibility with Canadian ASL teachers for future work. I designed the study with the guidance and support of two representatives from the SLIC (at that time the Sign Language Instructors of Canada) whose activities had largely stalled for nearly a decade and they were seeking to renew interest, momentum, and membership to the organization. Thus, our collaboration served the dual purpose of providing me with an empirical foundation about the landscape of ASL teaching in Canada and the SLIC with an opportunity to canvas potential members to inform future programming.

The study was guided by the following questions based on Reviere's (1996) needs analysis framework:

1. What is the profile of Canadian ASL teachers? 
2. Are Canadian ASL teachers aware of the SLIC's and/or the ASLTA's existence, mandate, and programming?

3. To what extent do teachers know about and participate in ASL teaching-related professional development, e.g., workshops, courses, conferences, and certification programs?

4. To what extent do teachers collaborate with other ASL teachers within and outside of their program?

To address these questions, the SLIC representatives and I developed an online bilingual/bimodal English-ASL survey (see Appendix B for a sample screenshot of the survey format) with questions about teachers' demographics, experience, and formal qualifications, as well as about teaching conditions and professional development and networks (see Appendix C for full survey). The survey yielded both quantitative data (Likert-type scales) and qualitative data (open-ended comments). Follow-up semistructured interviews (see Appendix D for interview question guide) were conducted in ASL and English, depending on the preference of the participant, and then translated and transcribed into English. The data was analyzed using descriptive statistics and qualitative coding. Findings were reported in the form of a brief report intended for publication on the SLIC website and as a journal article, both currently unpublished.

A total of 156 survey responses were received; of that number, 51 were complete and valid and passed the screening questions; eight teachers participated in follow-up interviews. It is unclear what percentage of the total number of ASL teachers in Canada these figures represent or if it is a representative sample; nonetheless, the responses yielded ample insight. I discuss several of the key findings below. 


\section{Teacher demographics}

ASL teacher-participants reported worked in colleges and universities (many elsewhere, as well) coast to coast, with no one responding from Northern Canada, and were primarily women-identifying $(\mathrm{N}+31)$. The majority of survey respondents were over 40 years old (aged $41-55, \mathrm{~N}=24$; aged $55+, \mathrm{N}=19$ ). Ten teachers had 10 years of experience or fewer (20\%), 19 between 11-20 years (35\%), and the remaining 21 teachers (41\%) had been teaching ASL for over 20 years. One interview participant had come out of retirement to ensure the program in his area would not close. Other interview data pointed to the profession being in a precarious state as ASL teachers are retiring faster than new teachers are joining the profession. Interviewees hypothesized that young deaf people are simply not interested in teaching as higher education and more and more secure professions have become more accessible.

Data from Gordon and Harvey's (2009) report on the status of ASL teaching in the province of British Columbia likewise suggested a teacher shortage. Goertzen (2018) suspected that the lack of deaf youth becoming involved in ASL teaching in Manitoba was because of the closure of the province's teacher training program and the precariousness of ASL teaching work. Goertzen identified this as a concern for the future of ASL teaching, especially because the younger generation of deaf people tends to be educated in mainstream settings, and so already have fewer opportunities to become proficient ASL users. This trend suggests a need for improved strategies to build a more stable and sustainable workforce. A strong workforce would likely help (or imply the need to) address the recurrent issues of last-minute hiring of inexperienced, underqualified teachers to fill rapidly expanding programs and, conversely, the inability 
for programs to stay open or expand due to a lack of viable teachers. The latter in particular was reported to possibly impact enrolments, as students may be discouraged by limited program offerings (i.e., no major, no upper-level courses) and waitlists resulting from having only one full-time instructor (Gray, 2018). The question of teacher qualifications is discussed further in a later section of this chapter.

The survey did not ask whether participants were hearing, hard of hearing, deaf, coda, etc., but did inquire about years using ASL and involvement with the deaf community, which were considered more relevant constructs at the time. In hindsight, this was a missed opportunity. A majority of teachers indicated using ASL for over 20 years $(84 \%, \mathrm{~N}=43)$ and only seven teachers $(14 \%)$ have been using ASL for under 20 years (the majority for between 6-10 years). The majority of respondents indicated they were "quite" to "very" involved with the deaf community $(87 \%, \mathrm{~N}=44)$, while seven (14\%) indicated they were "a little bit involved". No one indicated no involvement at all with the deaf community.

Given that involvement with the deaf community is widely considered important in ASL teaching, this latter question likely carried some social desirability bias, where respondents may not have wanted to identify themselves as not being involved. Interestingly, in McDermid's (2009) study of the experiences of deaf and hearing faculty in Canadian interpretation and pre-interpretation programs, a majority of deaf faculty were members of a deaf organization (e.g., local deaf club) and few were members of professional interpreting organizations; the opposite was true for hearing faculty. My survey results did not show this trend, likely because the teachers in this study were not involved in interpreter education programs where there is a well-recognized professional 
association; for teachers outside this context deaf cultural organizations are effectively synonymous with professional associations, as they lead teacher development discussions and activities.

Over three quarters of teachers who participated in this study $(\mathrm{N}=37)$ taught ASL fewer than 14 hours per week, eight taught between 15 and 29 hours per week, and only four were employed full time, i.e., over 30 hours per week. A total of 81 institutional positions were claimed by the 51 participants, which indicated that a high number of teachers were employed in multiple contexts. Goertzen's (2018) study of Manitoba ASL teachers likewise identified that many teachers work in multiple settings, often at once, including private courses, community education programs, and in public high schools.

The teachers interviewed in Doré (2015) suggested that ASL teaching is precarious as positions appear and disappear with student demand, although the work is not uniformly in/secure: well-established multi-year programs (e.g., interpretation programs) and those in larger cities may offer better job security for teachers than smaller programs in less populous areas. One interviewee shared that she might move to a neighbouring city to continue teaching due to dwindling course enrolments in her area. Another interviewee reported commuting up to several hours and across provincial borders to take on enough supplemental teaching work to make a living. This finding helped frame the rest of my explorations of ASL teachers by serving as a reminder that regardless of motivation, realistically, teachers may not have the time, finances, or incentive to pursue extensive or intensive training to improve or standardize their practice. Any proposed professional development program should take this reality into account. 


\section{Awareness of professional associations}

This survey asked about teachers' awareness of the two national sign language teachers' associations, the SLIC and the ASLTA. Five survey participants were SLIC members, most of them from either Eastern or Western Canada. In Central Canada, roughly one third of participants were very familiar with the SLIC. Interestingly, four of five ASLTA members were in Central Canada. Ten respondents had never heard of the SLIC at all, and another five knew it by name only. There were no notable trends regarding awareness of or membership to the SLIC or ASLTA by institution, geographical or institutional location, etc. It was notable, however, that interviews showed two sides of awareness of SLIC: teachers had either never heard of it at all, or teachers had heard of it, but had no knowledge of recent activity. Comments for the former included "I wish I knew about them years ago" and "[I had never heard of SLIC] until I opened the survey". Those who were familiar with SLIC expressed recurrent concern about the organization not informing the public of their presence in recent years: "WHERE IS SLIC?" and "I haven't heard anything from them for years".

Thus, although the survey data indicated moderate awareness of SLIC, interviews clarified that awareness does not necessarily translate into sustained knowledge of (or participation in) its activities. These comments made it clear that the SLIC has significant rebuilding to do before it can be a uniting force in uniting teachers Canada-wide, a voice for ASL teacher advocacy, or a provider of professional development programs.

\section{Participation in professional development activities}

The survey found that participants highly valued professional development $(96 \%$ responses "quite" to "very important") for all areas of ASL teaching (classroom 
management, deaf history and culture, ASL linguistics, etc.) and were unanimous in wanting to complete more professional development. Yet, there was great diversity in actual participation in activities such as workshops, conferences, peer observation, and individual research. Participants also reported diverse formal training backgrounds with no observable trends in relation to traits such as years teaching and number or type of qualifications. Nearly $80 \%(\mathrm{~N}=38)$ indicated having completed ASL-related certification, including curriculum-specific certificates (e.g., CHS’ Signing Naturally instructor certificate), certificate programs offered by SLIC or the ASLTA (e.g., ASLICE, and university degrees (e.g., Bachelor's in ASL). There were seven Master's degrees related to ASL, ranging from applied linguistics and language acquisition to deaf education. This finding was in line with anecdotal reports that ASL teacher qualifications in Canada are far from standardized.

According to the survey, the main barriers to teachers' participation in professional development activities were that it was too expensive and too far and expensive to travel. As one respondent said, "ASL teaching is usually offered part time, minimal pay - taking training costs money and it does not help with the minimal pay”. Other barriers included a lack of suitable options and conflicts with work schedules.

Regarding collaboration, participants had a wide range of experiences. Some types of collaborative activities were more popular than others-for example, many participants indicated frequently or fairly frequently sharing teaching materials ("often/always", $\mathrm{N}=22$ ) and discussing teaching tips ("often/always", $\mathrm{N}=19$ ), but far fewer joined classes or team taught with another teacher or contributed to ASL blogs or chat rooms. Notably, a significant majority of respondents indicated wanting to know (N 
$=45)$ and be more connected to $(\mathrm{N}=49)$ other ASL teachers, and yet 34 teachers also indicated feeling like they were a part of an ASL teaching community. One possible interpretation was that teachers were creating highly localized teaching communities within their programs and so felt supported on a day-to-day basis, but wanted to extend their connections to other programs and larger-scale communities.

A related but unexpected barrier to both professional development and collaboration was expressed in the interviews - specifically, expressions of exclusion, where some teachers felt they were left out or pushed away by their professional community. This sentiment was expressed by teachers of different hearing and sign language profiles, including: a sign language first language user who was coda and several ASL second language users who variously identified as hearing, hard-of-hearing, and late deafened. Teachers described their exclusion in different ways, for example: they were not perceived as competent or "serious teachers" by deaf colleagues, they were overlooked for job opportunities, they were not invited to take part in decision-making (e.g., curriculum development), and their applications to participate in workshops and other training opportunities were ignored or rejected. The following excerpt from one teacher's follow-up interview said it well:

There seems to be a major lack of information. It could also be related to the fact that I'm kind of outside the deaf community, too, so I feel like I'm missing some of this information. I wonder sometimes if that's a big obstacle. 
A second teacher echoed:

Because I've been faced with the reaction from a deaf teacher so many times I just want to stay in my bubble and do what I do. I don't want to be faced with the whole, Yes, I teach sign language; yes, I'm hearing. So, yeah, I've been tempted and there have been some really interesting conferences I would have liked to attend. But no, I never have.

These stories gave preliminary significant insight into not only some of the ways that teaching as a profession is organized, but also potential complexities of ASL teachers' pathways to and through the profession, particularly as related to questions of language and identity (e.g., hearing/deaf/other, language background). It was a reminder

of the need for my dissertation to pay attention to individual stories of experience.

Having described the main findings of Doré (2015), below I turn my attention to literature about ASL teaching — in particular, standards and studies that have aimed to support and regulate ASL teachers and their professional practices.

\section{Early teacher standards}

Looking at ASL teaching across North America, scholars have observed and lamented the common practice of hiring ASL teachers for their signing abilities without or with little linguistic knowledge and/or teaching experience (Cooper et al., 2008; Kelly, 2001; McKee, Rosen, \& McKee 2014; Rosen, 2010; Smith, 1988). Some ASL programs have foregone expansion (adding more courses, more levels, etc.) as a result of a lack of qualified teachers (Gray, 2018), but many have not. The hiring trend has largely continued despite the establishment of common standards and training programs and ASL program administrators' expectations for a wider range of skills beyond sign 
language proficiency, including knowledge of ASL linguistics, deaf culture, and language pedagogy (Cooper, Reisman, \& Watson, 2011).

The implications of this combination of factors were various:

... [the] quality of training for ASL teachers [is questionable]... [Teachers] do not have a strong understanding of the theoretical, empirical, and pedagogical assumptions the various curricula have about L2 teaching and learning. Each of the ASL curricula rest on a variety of linguistic, learning, and pedagogical assumptions, which have transformed over time. Some teachers used ASL curricula that subscribe to older assumptions, while other teachers used ASL curricula subscribing to recent assumptions. There are inconsistencies in curricula used by teachers of ASL. Pedagogical practices are often created by 'gut' feelings, not scholarly, systematic understanding of what curriculum is, and what teaching entails. Inconsistencies in the selection of curricula used by L2 ASL teachers raises questions about the understanding of the teachers of the principles and practices in second language curriculum development and instructional strategies. The teachers need to understand the assumptions that guide the development of curriculum. More specifically, teachers need to acknowledge that curricula differ in the selection of topics, types of linguistic structures, and the degree of emphasis on vocabulary, grammar, and culture information in teaching and learning. (Rosen, 2017, p. 22)

To raise the profile of ASL teaching and encourage teachers to standardize their skills, several scholars put forward criteria that ASL hiring committees should consider when hiring instructors. Early scholars Kanda and Fleischer (1988), for example, argued 
that teachers should have a "proper" attitude towards sign language and deaf people and linguistic and cultural competence ideally derived and maintained through significant interaction with the deaf community. Ideally, say Kanda and Fleischer (1988), an ASL teacher should also "be educated, demonstrating knowledge and application of educational and pedagogical principles along with formal study of the language" (p. 193). The ASLTA's current certification (as described in Chapter Two) still reflect most of these criteria, though with less emphasis on learning and growing professionally within the deaf community.

Ten years after Kanda and Fleischer (1988), Wilcox and Peyton (1999) proposed a set of criteria that put distinctly more emphasis on formal education and training and less on deaf cultural or community connections:

ASL classes should be taught by teachers who have a formal background in second language pedagogy, experience teaching ASL, and verifiable proficiency in ASL. Ideally the teacher or a co-teacher would be a native ASL user. Some schools require that teachers be certified by the American Sign Language Teachers' Association. (p. 159).

Wilcox and Peyton's criteria most likely reflected the progress that organizations such as the ASLTA had made by then in establishing centralized, standardized research-based criteria. Like Kanda and Fleischer, Wilcox and Peyton also did not imply that all teachers should be deaf and instead highlighted linguistic ability, which may suggest that deafonly hiring was taking a backseat to credentials.

In the 1990s, the ASLTA revisited its early teacher standards based on an extensive study conducted by Newell (1995abc), which I describe in the next section. 


\section{Job Analysis of Teaching American Sign Language (JATASL)}

To help inform revisions to the ASLTA's teacher standards, Newell conducted a survey-based longitudinal investigation of teachers' profiles (1995a) and perceptions of characteristics of qualities required for teaching ASL (1995bc). The study compared its findings from 1993-1994 to those from an earlier study, also of teachers in the USA (Battison \& Carter, 1980; for the National Association of the Deaf). The comparison allowed Newell to make some conclusions about the evolving state of the profession. Most notably, Newell's study was possibly one of the first attempts at integrating empirical data on ASL teachers' perceptions of qualifications into a system of standards that would inform a professional development certification and training program.

Newell's survey (1995abc), called the Job Analysis of Teaching American Sign Language (JATASL), comprised five sections about skills and qualifications and one background section. There were 359 ASL teachers from across the USA who responded to the JATASL. Some findings were similar to the findings of Doré (2015) about Canadian teachers; for instance, the vast majority of Newell's participants reported teaching part-time $(\mathrm{N}=234)$ and at multiple levels in multiple types of institutions (colleges, universities, community programs, etc.). Most findings were significantly different, however. The participants in Newell's study had a wider range of teaching experience (3-5 years, 6-9 years, 10-14 years, and 15+ years each had 20\% representation) and significantly more formal education (nearly $80 \%$ had Bachelor's degrees or higher).

Newell (1995a) commented that his study's figures were similar to Battison and Carter's (1980) findings 13 years earlier. One notable difference in the two studies' 
findings was in hearing status: Newell (1995a) found that $50 \%$ of respondents identified as deaf, 45\% hearing, and 5\% "other" (including "hard-of-hearing" and "coda"); Battison and Carter (1980) found that $76 \%$ of their participants were hearing and $24 \%$ deaf. Doré (2015) did not ask respondents to identify their hearing status; thus, comparisons cannot be made.

Beyond profiling teachers, the JATASL collected teachers' perceptions of the competencies needed to teach ASL according to five areas of knowledge and skills (Newell, 1995b):

1. Knowledge of educational foundations, about general issues of teaching, learning, and educational policy;

2. Linguistic structure of ASL, about specialized knowledge e.g., grammar and lexical rules;

3. Methods of teaching, about methods and strategies for teaching ASL and deaf studies;

4. Professional knowledge, about theories of second language pedagogy, classroom management strategies, etc.; and

5. Bilingual skills, about communication skills in ASL and English.

There was strong consensus on nearly all items of the JATASL and no significant differences between groups (based on age, gender, education, etc.), which is in itself a significant finding (Newell, 1995c). The most highly rated items were linguistic knowledge, ASL proficiency, and knowledge of deaf history and culture, while items under "professional knowledge" were rated as "moderately important" (Newell, 199b). 
Highly theoretical knowledge, such as curriculum theory and philosophy of education, were rated the lowest.

After the ASLTA proposed its new standards, there have been a small number of studies that have more considered more recently established ASL teacher standards, as described next.

\section{Recent teacher standards}

A Canadian study about ASL teacher standards that deserves mention is Goertzen's (2018) survey-based study that examined, among other things, ASL teachers' perceptions of the most important knowledge and skills required to teach ASL. Although her research included only a small sample of ASL teachers in the Manitoba secondary school system $(\mathrm{N}=11)$, most of her participants reported working in several settings, including post-secondary, and so her findings are worth mentioning here. In her survey, she asked her participants to rank teaching skills and knowledge on a scale from 1-10. A corresponding score of 1-10 was assigned to each response and results were tabulated as the sum of scores (see Table 1).

To summarize, ASL proficiency (score: 74) was the top-rated item, which Goertzen inferred meant native users based on her knowledge of the Manitoba deaf community. The second highest rating was ASL instructions/curriculum familiarity with lesson plans (score: 69), to which Goertzen commented that most of the teachers were familiar with the Signing Naturally curriculum, which includes lesson plans. Deaf studies (score: 55) was rated as the third most important for ASL teachers, which highlighted "the desire to share Deaf history and traditions as part of ASL courses" (Goertzen, 2018, p. 59). Goertzen noted that the knowledge and skills rated as least important, classroom 
management and teaching principles, are those emphasized most in public high schools. Goertzen's findings, particularly about the lowest-rated items, may have been a reflection that the practice of hiring inexperienced teachers and relying on ready-made curricular materials like Signing Naturally also apply to the Canadian context.

\section{Table 1.}

Sum of Scores for ASL Teacher Knowledge/Skill Items (from Goertzen, 2018, p. 58)

Item

ASL Proficiency

ASL Instructions/Curriculum Familiarity with Lesson Plans

Deaf Studies

Lesson Planning

Cognitive and Learning Theory

ASL Instruction/Curriculum Familiarity without Lesson Plans

Record Keeping

Assessment/Evaluation

Classroom Management

Teaching Principles

\section{Sum of Scores}

74

69

55

52

50

48

39

38

35

28

In the USA, Jacobowitz (2007) reviewed different standards for ASL teachers put forward by language and education organizations. Of the six organizations she examined, only the ASLTA had standards specifically for teachers of ASL in higher education, which were those that Newell's (1995abc) aforementioned study helped to inform. At the 
time of Jacobowitz' review, the ASLTA had a three-tier evaluation system based on those standards. The current two-tier system was described earlier in this chapter. To achieve the first tier in the three-tier system, teachers sent an application package including evidence of teaching knowledge and a video demonstrating ASL proficiency; the second tier required teachers to provide answers to ASL linguistics questions; and the third tier involved a live interview to respond to knowledge questions and provide evaluations of sample student work (Jacobowitz, 2007). Jacobowitz (2007) observed that there was no mention of English proficiency requirements, despite English being the dominant language of written curricular materials, research publications, and communication at most institutions. I also observed that in the ASLTA's system there was no mention of deaf or hearing status or membership to or participation in any deaf organizations in the evaluation.

Although ASL scholars and teachers' associations have proposed standards and guidelines for ASL teachers, teachers may not all want to or be able to access those opportunities for a variety of reasons. The following section presents some of the scholarship in this area.

\section{Barriers to professional development}

A small number of studies (including Doré, 2015) have addressed the reasons that teachers do or do not participate in professional development activities. Fox (2010) examined the reasons that teachers in the USA choose to apply or not for the ASLTA certification specifically. Her survey-based study included ASL teachers $(\mathrm{N}=138$; from K-12 and higher education and teaching ASL as a first and second language) who were members of four professional organizations, including the ASLTA and several 
interpreters' associations. She considered correlations between seeking the certificate and demographics, training background, and attitudes about the benefits and costs of certification. Her findings suggested that teachers are deterred by such barriers as cost and lack of mentorship, despite the incentive that some program administrators uphold ASLTA standards-based hiring (e.g., Gray, 2018). Fox (2010) proposed that more varied levels of credentials — e.g., entry level certification as well as advanced — and a phase-in cost structure might improve buy-in.

According to Fox (2010) barriers to professional development included cost and lack of support from peers and employers. Participants in Doré (2015) likewise pointed to time and cost as deterrents to participating in development activities, particularly since many of them are teaching part-time. Looking more broadly, however, there can also be systemic barriers to professional development for deaf teachers in particular. Atherton and Barnes (2012) investigated the impact of a British mandate that all current and incoming foreign language teachers complete mandatory pedagogical training, including British Sign Language (BSL) teachers. The study found that the training was not deaffriendly (e.g., heavy reliance on English, spotty interpretation) and created unnecessary barriers to deaf BSL teachers' completion of the training.

Goertzen (2018) also found that ASL teachers in secondary school settings faced a similar issues as the teachers in Atherton and Barnes' (2012) study because the Manitoba Ministry of Education required its teachers to hold a teaching degree, which few deaf instructors held. This requirement pushed programs to cancel courses, hire a certified hearing teacher to accompany the uncertified teacher, or hire hearing instructors to teach the course, which was widely frowned upon (Goertzen, 2018). 
Other barriers to ASL teachers' professional development included audism at work. Schornstein (2005) reflected on her experiences as a deaf child of being left out of conversations with her hearing relatives as well as missing out on what she referred to as "incidental information", i.e., information gleaned from overhearing conversations. She felt this situation carried into her professional life, where she found it difficult to jump into conversations with hearing colleagues without an interpreter and significant shifts in discourse practices (e.g., speaking slowly, clear turn-taking).

McDermid's (2009) study examined the experiences of deaf and hearing faculty in Canadian interpretation programs to understand the relationships of power at play in the professional setting. He found several fundamental conflicts between the two groups. Deaf faculty were much more collectivist in that they sought frequent input on their pedagogy from the deaf community as well as to "give back" to the deaf community frequently through involvement with, for example, deaf youth summer camps. Hearing faculty, meanwhile, were much more involved in academics (e.g., research papers) and professional organization activities (e.g., conferences), and engaged less with deaf community activities. Overall, several of the deaf faculty felt isolated in their programs, or "alone doing our thing" (McDermid, 2009, p. 235) and fundamentally conflicted about their responsibility to their students and the deaf community the students would eventually serve as interpreters. McDermid (2009) described "unchecked audism" (p. 244) with deaf faculty being treated as "sub-altern" to hearing faculty and he recommended changes to organizational structures and internal practices to bring greater equality to the workplace. 
Gray (2018) similarly found that deaf ASL instructors in higher education reported awkward interactions with hearing colleagues, or they were balked at for requesting interpreters, intentionally excluded from conversations, and required to empirically justify teaching decisions. Gray's participants also reported audist behaviours by students toward their ASL instructors, including talking in class and criticizing the aesthetics of the instructor's signing. While the instructors had overall good treatment by upper administration, they also faced ongoing daily challenges such as needing to defend the hiring of new deaf faculty over hearing candidates and fighting for equipment and lab space. To combat the attrition that some of these issues provoked, Gray's participants reported mentoring newcomers through things like meeting regularly, running through exercises together, classroom observations, and providing materials and resources (p. 7778).

Looking beyond ASL teaching, there have been several studies about deaf faculty members' experiences of discrimination in the workplace. These studies were useful in framing the present study in that they gave a broader lens on the deaf experiences in the workplace. For example, Woodcock, Rohan, and Campbell (2007) studied the experiences of deaf academics and found that the cost and hassle of arranging for interpreters can mean that faculty opt out of meetings, trainings, research, and even some classroom exercises. Also, when interpreters are provided, they are not always wellversed in the material and thus may result in inaccurate interpretation. Smith and Andrews (2015) identified "fear factors" for deaf and hard of hearing faculty in higher education, including: the difficulty of arranging for accommodations to attend interviews, trainings, events; potential resentment from colleagues and the administration about the 
cost burden for ongoing support services; self-isolation due to the difficulty of developing collegial relationships; changes to the job structure that would require modifications or reductions to the role; and the potential for lawsuits, not for lack of accommodation but for lack of support and mentoring. Pine (2012) pointed to many of the same concerns in her study of deaf tenured faculty.

In closing, the theoretical framework described in the first part of this chapter provided a lens and a language for understanding how stories are told within social contexts that enable and constrain their telling (see Figure 1). These theories helped to frame the social, temporal, and spatial interactions between teachers and their various contexts of practice, including their professional and their cultural and linguistic communities. The studies of ASL teachers described in the second part of the chapter gave a window on teachers' backgrounds as well as some of the context (including grand narratives) that potentially enabled and constrained their pathways to, through, and forward in the profession. The present dissertation study built on this theory and scholarship using data collection and analysis procedures that are described in the upcoming chapter. 


\section{Chapter Four: Methods}

[A] method is a set of procedures for collecting and analyzing research data. A methodology, on the other hand, is broader: a methodology is a method plus an underlying set of ideas about the nature of reality and knowledge. (Smart, 2007, p. 56)

Following Smart and having discussed the theory that informs narrative inquiry as a methodology in Chapter Three, in this chapter, I provide details about the procedures of data collection and data analysis that I undertook in response to this study's research questions:

1. What are ASL teachers' pathways to the profession?

2. What are their pathways through the profession?

3. What are their pathways forward in the profession?

The first section below provides an overview of this chapter.

\section{Overview}

Following clearance by the Carleton University Research Ethics Board (CUREB; clearance \# 106640; see Appendix E) the present study unfolded in two roughly concurrent phases. An overview of the methods is presented in Figure 2. Briefly, in one phase, which I call Storying, I conducted multi-part interviews with ASL teachers about their professional experiences. I coded the interviews informed by the narrative scholarship described in Chapter Three, particularly by the narrative analytical approach promoted by Clandinin and Connelly (2000), described later in this chapter. The resultant findings took the form of the narrative portraits and stories presented in Chapters Five to Eight. In the other phase, which I call Mapping (an extension of the landscape metaphor), 
I collected data from a range of sources, including interviews with ASL program supervisors and deaf cultural organization representatives and publicly available web data. The Mapping data did not respond directly to the research questions; rather, together with the background established in Chapter Two, the Mapping data was used to contextualize, support, expand, and contest my interpretation of teachers' stories from the Storying phase by providing the perspectives of alternate stakeholders. In Chapter Nine I summarize the findings and discuss the key themes that arose from the findings and their implications in relation to the goals of the study.

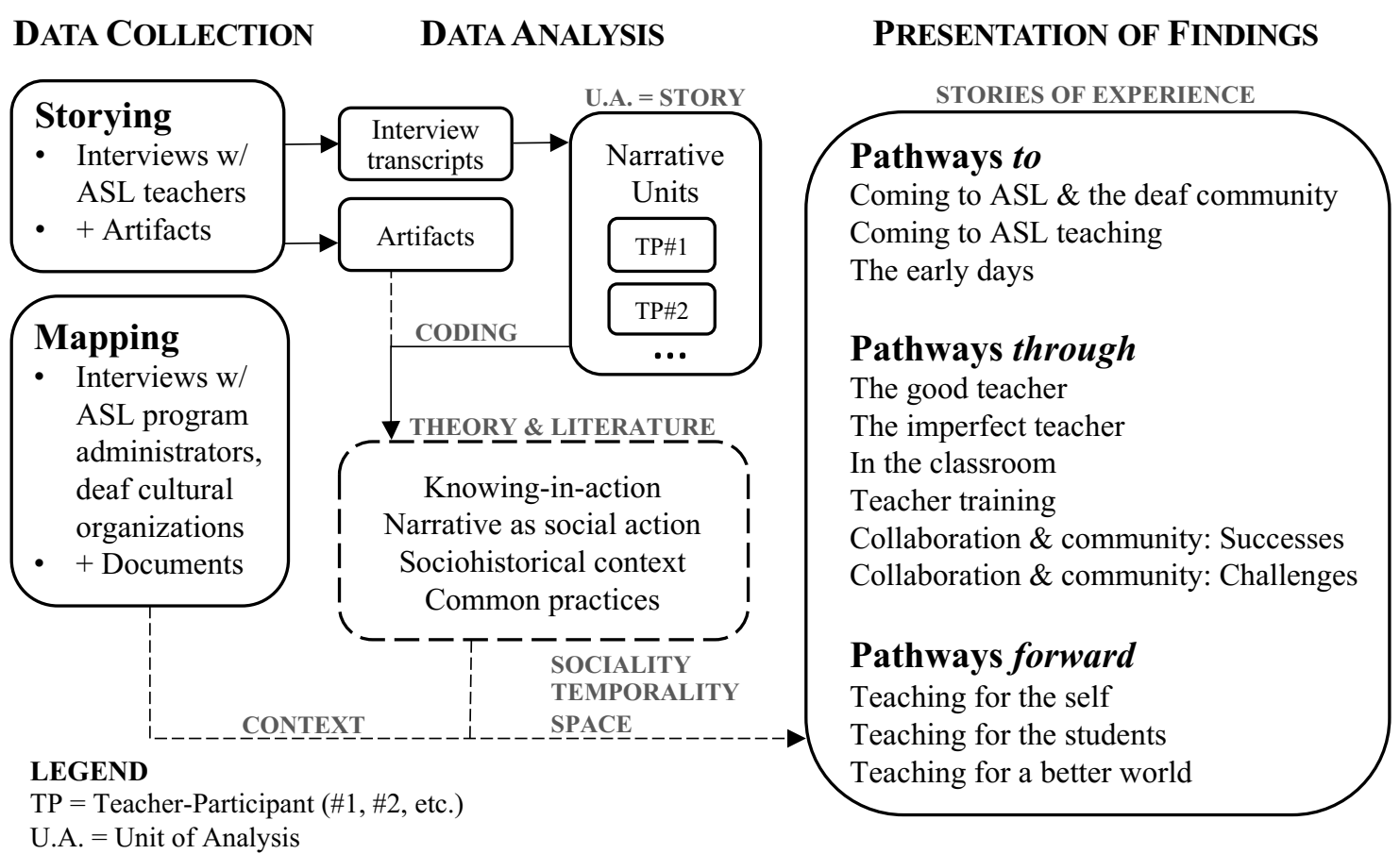

Figure 2. Illustration of data collection, data analysis, and presentation of findings.

Although I had a general timeline and structure for the present study, the methods were ultimately emergent, where, to a large extent, the procedures unfolded in an open- 
ended way. This approach was necessary for two main reasons. The first reason was anticipated: little data has been formally collected and published about Canadian ASL teaching, and so I wanted to ensure methodological flexibility at the outset to accommodate unexpected discoveries. The second reason became clear only in hindsight: my projected path through this research took detours that demanded some level of adaptation and refocus. In brief, as mentioned in Chapter One and revisited in Chapter Ten, I entered this study expecting to continue collaborative efforts with the SLIC, where the representatives were going to play a significant role. Partway through, however, our collaboration collapsed, which redirected more of my attention to the teachers who became primary collaborators through their storytelling. My ethics clearance allowed for this shift.

This research is deeply qualitative. At no point do I suggest that my interpretations are objective or anything but filtered through my own positionality, prior experiences, and relationships with the participants and surroundings. My selfintroduction in Chapter One was an essential part of providing the reader with some sense of what beliefs, values, assumptions, and experiences I came into this study with as a researcher and active participant throughout the study. This statement of positionality is especially important to the orientation of my research in which "local and specific constructed realities" (Guba \& Lincoln, 2004, p. 24) and situated truths (Riessman, 2008) were created as a result of the interview dynamics and my narrative interpretation that ultimately created an embodied and relative living history.

In other words, I wrote this dissertation with the full recognition that it will be but a snapshot in time, with conclusions drawn from particular interactions with particular 
participants at a particular time in a particular space, where much was learned although the captured reality was fleeting. I recalled Cleary's (2013) advice: nothing stands still. Cleary used this phrase to point to postcolonial theories of multiply positioned identities that recognize the dynamic nature of identity. To tell myself that nothing stands still helped to keep the ethical issue of representation in research at the fore-namely, it helped me avoid the temptation to inadvertently romanticize or pity (to use Cleary's terms) participants in order to fit the storyline - a risk in narrative work (Gubrium \& Holstein, 1999).

As I described in Chapter Three, stories are edited throughout their telling, between tellings to different people or in different situations, each time performing its own social function, each its own call to membership or nonalignment, to reflection or assertion, and so on (Gubrium \& Holstein, 1999). This was an important consideration in the present study, where I was not an ASL teacher myself, nor heavily involved in deaf and signing communities in Canada, and so I relied on these edits to point me to what the story is doing and what other possible or impossible stories are connected, but untold (so far). Moreover, in the several years of this dissertation, the Canadian sign language landscape has shifted on the small and large scale. For example, as described in Chapter Two, in 2019 the Government of Canada officially recognized sign languages (ASL, LSQ, and ISL) as the official languages of deaf people in Canada (Bill C-81). This alone has most likely already changed the landscape and many of this study's teacherparticipants' pathways, particularly those involved in activist work.

Finally, although the subject of this research was sign language and several participants were deaf and active members of their local deaf communities, this study was 
not developed as a critical or transformative research study. In other words, I had guiding questions, theories, and pre-conceptions, but I did not seek specific results with a social action agenda (Creswell, 1998). I was, however, informed by principles for ethical research with deaf people and communities (e.g., Foster, 1987; Pollard, 1992; Singleton, Jones, \& Hanumantha, 2014), although how the key aspect of ethical research, collaboration, changed over time as my collaborators changed from the SLIC representatives to ASL teachers themselves.

In the next sections I describe the study's participants, instruments, procedures, and analysis according to two complementary phases, Storying and Mapping, and how I brought together the findings from both.

\section{Storying Phase}

The Storying phase consisted of multi-part interviews with seven ASL teachers from across Canada that were analyzed using qualitative narrative coding (Saldaña, 2013) informed by Clandinin and Connelly's (2000) three-dimensional analytical framework: sociality (intrapersonal and interpersonal interactions), temporality (past, present, and future), and space (place, events). The sections below explain each element in more depth.

\section{Participants}

To identify potential ASL teacher-participants, I used my scan of post-secondary institutions from Doré (2015; see Appendix A) as a starting point to direct me to colleges and universities with ASL programs and, therefore, ASL teachers. I used publicly available email addresses to reach out to ASL teachers with an invitation to participate (see Appendix F). I also relied on snowball sampling where ASL teachers were invited to 
forward the invitation to peers (a script was provided to ensure accuracy of information in third party recruitment; see Appendix G). A total of seven ASL teachers from across Canada volunteered to participate in the study. I provide an overview of participants' profiles in Table 2. These profiles provide basic demographic and background information, a snapshot of the participants in this study, for the purposes of clarifying the methods of the study. The profiles are amplified into narrative portraits in Chapter Five, where I develop a more complete and in-depth account of the teachers' professional lives based on their interviews.

Of the seven teachers, two distinct groups emerged: novice and experienced. I did not draw a hard line around novices and experts, nor did I consider much of the established literature on the topic wholly applicable to ASL teaching. In general education, a teachers' life cycles will likely include formal pre-service training, a regulated curriculum, structured in-service training, and a coherent body of knowledge. In ASL teaching, there is little evidence to suggest that any of these apply, at least not in any meaningful or standardized way. Instead, I looked to this study's ASL teacherparticipants and identified two groups, novice and experiences, based on years of experience. Nathalie, David, and Nick had a year or two (or less) of experience under their belts and for the most part were not yet very active in ASL teacher networks or training. Ken, Michelle, Erica, and Donna, meanwhile, had several decades of experience and a high level of involvement with ASL teacher networks locally and beyond, with the exception of Michelle who worked mostly in isolation. I made the distinction between novice and experienced for the teachers in this study recognizing that the distinction 
would need to be revised and likely more thoroughly empirically supported in another study where the participants may not cluster so clearly.

There were a range of hearing and deaf identities present among the study's teacher-participants, including deaf and oral deaf (Nick, Ken, Michelle, Erica, Donna), deaf and hard-of-hearing (Nathalie), and hearing (David). The combined identities are to reflect that some of the teachers variously self-identified during our conversations and I made no attempt to force one identity label or the other.

As expected, based on Doré (2015) and prior literature about ASL teaching as a profession, this study's ASL teacher-participants had teaching experience in many different ASL teaching contexts beyond post-secondary foreign/modern language departments. The column "Current ASL Teaching Position/s" in Table 2 was included to give the reader context for the roles they held at the time of our interviews, but as the stories in Chapters Six to Eight will show, many of them have worked many other roles in other contexts over the course of their careers.

Each teacher participated in three interviews over one to three separate sessions lasting anywhere from one to three (and, in one case, more) hours each (see Table 3). The question guide described below and appended in Appendix $\mathrm{H}$ lent a degree of consistency and structure to the interviews, which were largely conversational and unstructured in order to maximize opportunities for teachers to share stories most meaningful to them. 
Table 2.

ASL Teacher-Participant Profiles

\begin{tabular}{|c|c|c|c|c|}
\hline $\begin{array}{l}\text { Name (Hearing } \\
\text { Status) }\end{array}$ & $\begin{array}{l}\text { Language } \\
\text { Background }\end{array}$ & $\begin{array}{l}\text { ASL Teaching } \\
\text { Background }\end{array}$ & $\begin{array}{l}\text { Current ASL Teaching } \\
\text { Position/s, Full-/Part-time }\end{array}$ & $\begin{array}{l}\text { Deaf Community } \\
\text { Involvement }\end{array}$ \\
\hline $\begin{array}{l}\text { Nathalie (hard-of- } \\
\text { hearing/deaf) }\end{array}$ & $\begin{array}{l}\text { English L1; ASL L2 } \\
\text { (young adult) }\end{array}$ & $\begin{array}{l}\text { Novice }(<5 \text { years }) \\
\text { CTESL* }\end{array}$ & University instructor, part-time & $\begin{array}{l}\text { Increasingly involved } \\
\text { (e.g., sports) }\end{array}$ \\
\hline Ken (deaf) & $\begin{array}{l}\text { MSL L1; ASL L2 } \\
\text { (youth) }\end{array}$ & $\begin{array}{l}\text { Experienced ( } 20+\text { years) } \\
\text { Extensive ASL teacher } \\
\text { training (workshops, } \\
\text { courses, mentorship) }\end{array}$ & $\begin{array}{l}\text { Self-employed teacher, } \\
\text { facilitator, and consultant, full- } \\
\text { time }\end{array}$ & $\begin{array}{l}\text { Very involved; leadership } \\
\text { (e.g., cultural } \\
\text { organizations) }\end{array}$ \\
\hline David (hearing) & $\begin{array}{l}\text { English L1; ASL L2 } \\
\text { (young adult) }\end{array}$ & $\begin{array}{l}\text { Novice }(<5 \text { years }) \\
\text { CTESL* }\end{array}$ & University instructor, part-time & $\begin{array}{l}\text { Somewhat involved (e.g., } \\
\text { social events) }\end{array}$ \\
\hline Michelle (deaf) & $\begin{array}{l}\text { English L1; ASL L2 } \\
\text { (young adult) }\end{array}$ & $\begin{array}{l}\text { Experienced ( } 20+\text { years) } \\
\text { Extensive ASL teacher } \\
\text { training (workshops, } \\
\text { courses, mentorship) }\end{array}$ & $\begin{array}{l}\text { Self-employed teacher and } \\
\text { tutor, full-time; former } \\
\text { community course instructor }\end{array}$ & $\begin{array}{l}\text { Somewhat involved (e.g., } \\
\text { social events) }\end{array}$ \\
\hline
\end{tabular}




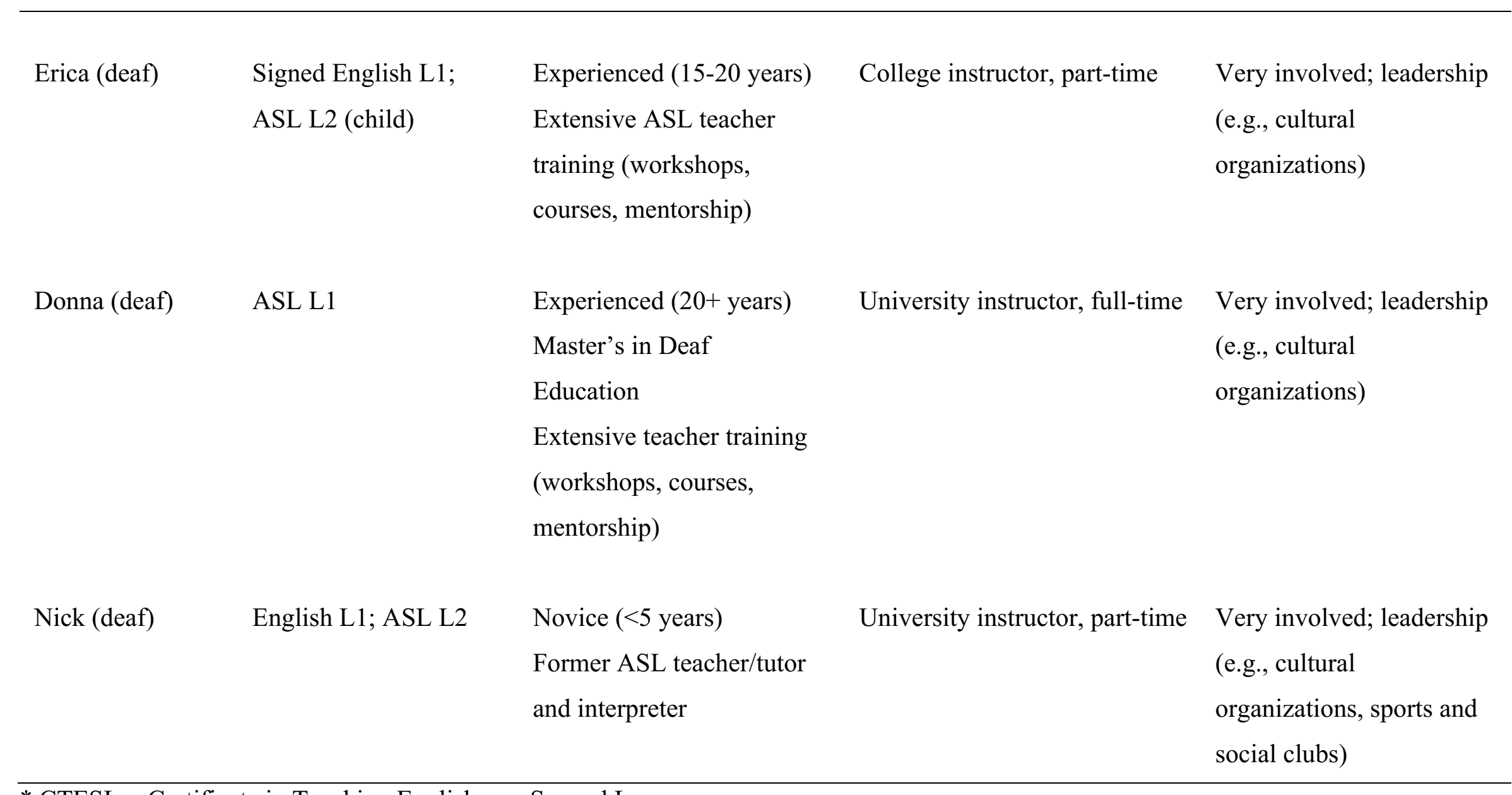

* CTESL $=$ Certificate in Teaching English as a Second Language 


\section{Instruments and procedures}

The week prior to the initial interview, I sent teachers a question guide (Appendix H) in preparation for our conversations. The guide was informed by Seidman's (2006) three-part life history interview structure: (1) life history (as far back as possible, he suggests), (2) details of the experience (e.g., "a day in the life"), and (3) reflections and looking forward. Seidman's approach was well-suited to inviting stories about all three narrative dimensions (sociality, temporality, space) across the past, present and future (i.e., pathways to, through, and forward). The first interview focused on teachers' personal and professional background, e.g., when they learned ASL, how and why they came to work as teachers. This interview was intended to understand the extent of teachers' professional memories as well as their language lives (Ellis, 2018) that informed the ongoing work. The second interview focused on their ongoing practices, including classroom practices and their collegial and other support networks. For many of the ASL teacher-participants this interview also drew out their relationship with their linguistic and cultural community, which, as described in Chapters Two and Three, are deeply intertwined with the profession of ASL teaching. The third interview focused on teachers' reflections on their work and their visions for the future. For example, looking back, what do they aim to accomplish in the future through their work?

Throughout the interviews I practiced active interviewing principles where I sought to "activate" teachers' narrative production (Holstein \& Gubrium, 1995) through such strategies as open-ended questioning, follow-up questions, and probing questions to encourage teachers to complete their thoughts. This was also a way of keeping myself 
present as an active collaborator in the interviews and in the research more generally (Gubrium \& Holstein, 1997). As Ellis and Berger (2003) suggested: researchers who use interviews should not focus solely on the outcomes - the words spoken by the interviewees - but should examine the collaborative activities of interviewees from which these outcomes are produced. (p. 469) This was also a reminder in my analysis not to overlook the impact of my presence on the data itself, on the interview, as well as throughout the interpretive process of data analysis.

Teachers were also welcomed--but not prompted-to share artifacts, as relevant (see Table 3). Some teachers directed me to public artifacts (e.g., favourite online resources), which were noted, copied, and/or bookmarked; others described, sent, or lent me physical books, pamphlets, handouts, and other documents (e.g., student evaluations) that they had developed or used throughout their teaching careers. These were valuable pieces of data, as they were often a physical testament to long ago individual and collective efforts, sometimes the direct product of the teachers' own efforts. 
Table 3.

ASL Teacher-Participant Interview Details

\begin{tabular}{|c|c|c|c|}
\hline Name & $\begin{array}{c}\text { \# Interviews } \\
\text { (\# Hours) }\end{array}$ & $\begin{array}{c}\text { Language/s of the } \\
\text { Interview/s }\end{array}$ & Artifacts \\
\hline Nick & One (3) & English and ASL & Photographs \\
\hline \multirow[t]{3}{*}{ Ken } & Three (3.5) & ASL & $\mathrm{CV} /$ resumé \\
\hline & & & List of workshops taught/attended \\
\hline & & & List of courses taught/attended \\
\hline Nathalie & Three (4.5) & English and ASL & None \\
\hline \multirow[t]{9}{*}{ Michelle } & Four $(8.5+)$ & ASL & $\mathrm{CV} /$ resumé \\
\hline & & & List of workshops taught/attended \\
\hline & & & List of courses taught/attended \\
\hline & & & Teaching materials (handouts, lesson \\
\hline & & & $\begin{array}{l}\text { plans, presentation slides, books, } \\
\text { etc.) }\end{array}$ \\
\hline & & & Training materials (e.g., manuals) \\
\hline & & & Resource book \\
\hline & & & Student evaluations \\
\hline & & & Photographs \\
\hline Erica & Two (3) & ASL & None \\
\hline \multirow[t]{2}{*}{ Donna } & Two (3.5) & ASL & Training materials (e.g., manuals) \\
\hline & & & Resource book \\
\hline David & Three (3) & English & None \\
\hline
\end{tabular}


In my commitment to supporting participants in shaping how their narrative was told, there were several participants for whom the interview process took an alternative route. For example, Michelle preferred to conduct interviews in person in her home and more informally over several meetings of one to five hours. In each of these interviews, Michelle preferred not to audio-/video record or it was not conducive to do so. Instead, each time I took copious field notes and photographs and collected teaching props, books, handouts, and more. In Nick's case, our "initial" interview extended over three hours and encompassed all three interviews' topics. Conversation drifted between the interview prompts and side stories relating to his experiences with the deaf community, his teaching and life ambitions, and so forth. As with Michelle's, Nick's interview disrupted the structure of the intended data collection process, but the roundabout approach contributed immensely to our rapport and ultimately enriched the exchanges.

A downside of these departures from the structure was that the consistency of data collection was compromised. In Nick's case, further interviews were forfeited, as at the time we deemed them unnecessary because so many topics across all three interviews had already been discussed. In hindsight, I could have adhered more strictly to the structure by ensuring several sessions of interviews, which may have yielded more insight and a more nuanced perspective on their professional lives. In the case of Michelle, not recording our interactions was out of respect for her preferences, but it meant I had insufficient verbatim narrative data to conduct in-depth narrative analysis. The result was a sparser presentation of Michelle in the findings chapters, which were intended to be primarily organized around verbatim narratives. I nonetheless attempted to make space 
for a discussion of her experiences based on my own interpretations of her unrecorded stories.

When all interviews were complete, I began the necessary early stage of preparing the data for analysis by interpreting ASL interviews and transcribing the interviews. I describe this process below.

\section{Interpretation and transcription}

I transcribed the spoken interviews and interpreted and transcribed the ASL interviews, in full, into written English in preparation for analysis. I recognized that interpreting sign language into written language was a complex and theory-laden interpretive process (Hole, 2007). In his own work, deaf scholar Ladd (2003) identified and celebrated the practical complexities of this task-different grammatical structures, the multi-layered and spatial nature of sign languages, and so on. Stone and West (2012) commented on the methodological and ethical challenges of sign language transcription:

Translation concerns itself with fidelity or the faithfulness of a rendering.

Frequently this can be understood as a 'neutral' rendering of a text in a 'source' language into its equivalent in a 'target' language. The discursive presence or

'voice' (Hermans, 1996: 5) of the translator is, however, often present and:

The resulting incongruities that open up in the text are due to the fact that, while we generally accept that translated texts are reoriented towards a different type of reader in a different linguistic and cultural environment, we expect the agent, and hence the voice, that effected this reorientation to remain so discreet as to vanish altogether. (Hermans, 1996: 5) 
... We have, all of us, acknowledged that there are multiple, complex and shifting ways of representing the voices of others. At some stage, authorial decisions are made. It is how those decisions are reached that makes the difference between an inappropriate, perhaps damaging representation of voice, and one which is reflexive, respectful and validated. (p. 648-649)

I was thus aware that doing this interpretation and transcription work involved making decisions for the deaf signers who participated in this study and that there were politics of power at play as I "filtered" the voices of the deaf teacher-participants.

With the above in mind, I opted to engage in the analytical and political work of translating ASL interviews into spoken English myself. To save time near the end of the process I obtained funding to hire certified ASL-English interpreters to help me interpret and transcribe the final transcripts. Although professional interpreters are bound to a code of conduct that includes confidentiality, they also signed a confidentiality agreement as part of this study. No conflicts of interest were reported. Importantly for my interpretive process, I translated and transcribed at least one of each teacher's interviews to ensure I had a strong hand in representing their narrative in written format before undergoing subsequent analysis. In the few instances where some aspects of the original ASL interview were unclear I contacted the participants directly for clarification.

Any translation is time-consuming; in my case it was additionally so due to the complex multi-modal nature of my data. Several interviews were a blend of spoken English and ASL, at least two of which were only audio-recorded with hand-written notes. Reconstructing the various pieces was thus a challenge. Early on, I attempted to streamline the process with NVivo (QSR International), a qualitative data analysis 
software that allowed me to code my audio-/video-recordings, do time-stamped transcription, collect, code, and sort other multi-modal data such as photographs, among other things. However, a technical glitch dismantled my annotation system and left three participants' transcripts permanently out of order and two permanently meshed, and so I abandoned the software. In its place, I devised a labour-intensive but more reliable speech-to-text process: I audio-recorded myself interpreting the ASL interviews aloud, making verbal note of e.g., laughter or gestures (shrug, facial expression, etc.), and then I transcribed the recordings in a Word document with an manual annotation system for laughter, pauses, gestures, etc. This step was the first of several opportunities to rework fuzzy translations (using the original video recordings for comparison) and flag and blur potentially identifying details.

At times, the teacher-participants used expressions that were more powerfully rendered in ASL than in English and so I used a simplified ASL glossing system of all capital letters in my transcript to indicate a one-to-one ASL sign-to-English word. Since I use glossing for one or two signs at a time and not full sentences or ideas, I opted not to follow a more formal glossing system (e.g., Supalla, Cripps, Byrne, 2017). Likewise, I also opted to transcribe interviews in the conversational tone in which they occurred, including laughs (e.g., [Laughs]), pauses (ellipses), false starts (e.g., "I just- I ...”), and colloquial contractions (e.g., wanna, gonna) in order to be more faithful to the participants' delivery.

Once transcripts were complete, I undertook the process of defining the unit of analysis, as described below. 


\section{Narrative units}

In this dissertation I refer to the unit of analysis as a narrative unit to reflect the narrative methodology of this study. Unlike in many other approaches to qualitative coding, I deemed inappropriate any definition of the unit of analysis that would lead to the deconstruction of teachers' stories into decontextualized segments, such as words, clauses, sentences, or lines of transcript. Clandinin and Connelly (2000) asserted that narrative analysis should have as its goal to represent "storied lives in storied ways, not to represent storied lives as exemplars of formal categories" (p. 141). This was in line with Vygotsky's (1962/2000) understanding of the unit of analysis, which "warned against the decomposition of the complex whole into elements" (as cited in Moen, 2006, p. 59) using the analogy of water into hydrogen and oxygen, where "neither of which possesses the properties of the whole and each of which possesses properties not present in the whole" (Vygotsky, 1962/2000, as cited in Moen, 2006, p. 59). Thus, in this study I considered a narrative unit to be a complete story, a coherent thought that was introduced, described, and concluded, and where further deconstruction would mean losing essential meaning.

Through the process of dividing the transcripts into narrative units, it became evident that some sections of the transcripts were information sharing rather than stories of experience unique to the person; rather, they were descriptions (dates, logistical details, etc.) of such things as training programs, events, and so on. These were not analyzed narratively but rather used for their contextual insight to inform my interpretations alongside Mapping data.

With transcripts segmented into narrative units, I then began the process of narrative coding, as described below. 


\section{Narrative coding}

I followed the multi-step coding process described in Saldaña (2013) to analyze the transcriptions' narrative units. In brief, the process included pre-coding and making preliminary jottings followed by the iterative process of coding, including: identifying codes, clustering codes into categories, and identifying themes and threads in the categories (Saldaña, 2013). The remainder of this section describes each of these terms and processes in more detail, although to say there was a hard line between these steps is inaccurate-I continually revisited the data, codes, and categories while unpacking the themes.

Pre-coding involved the unstructured, intuitive process of scanning the data and identifying "codable moments" (Saldaña, 2013, p. 19), such as particularly poignant quotes or segments of data. In my case, many of those "codable moments" became subheadings for the stories presented in Chapters Six to Eight. As sub-headings, they served to capture some fundamental emotion or reality of an experience in the storyteller's life (e.g., turning points, struggles and successes) and were intended to capture the reader's interest as they moved through the findings chapters.

Following pre-coding, I continued to develop my familiarity with the data through preliminary jottings, which Saldaña (2013) described as initial thoughts or "ideas for analytic consideration" (p. 20) that emerged in the early readings of the data and may become codes in the next stage of analysis. In this step, I documented my initial impressions and interpretations of the data, such as the general chronology or timeline of teacher-participants' professional lives, details of events and interactions, and so on. At this point, I was not yet directing my analysis toward my research questions or theoretical 
framework; rather, this was a loose process of capturing organic interpretations and connections, both mundane and significant, prior to the more structured coding processes that followed. I was also able to reduce the data somewhat at this point by flagging narrative units that were clearly peripheral to the study (e.g., small talk between myself and the teacher) were also put into a separate section and retained for later reference as contextual data to support other, more central stories.

The next stage of the coding process was to identify codes, which are "most often a word or short phrase that symbolically assigns a summative, salient, essence-capturing, and/or evocative attribute for a portion of... data" (Saldaña, 2013, p. 3). I used a combination of bottom-up codes, where I revised, added, and removed codes that arose through multiple readings of the data. To structure this stage of the coding process, I used Clandinin and Connelly's (2000) three-dimensional framework of narrative inquiry (described in more depth in Chapter Three): sociality (intra- and inter-personal interactions), temporality (past, present, future), and space (place). For example, I coded for who - interactions with students, colleagues, mentors, and so on, as well as teachers' reflections on their own professional lives; I coded for when - the sequencing and scaffolding of teachers' experiences, such as what events built on others; and I coded for where-locations that experiences unfolded (in the classroom, in the office, etc.) and the abstract locations (types of institution or organization) and their processes that may have facilitated or impeded teachers' experiences (e.g., policies, rules). I read and re-read the narrative units and attributed one or more codes that reflected these dimensions.

Having developed a deeper sense of the stories, I then used a top-down coding scheme to draw my attention to the background, theoretical concepts, and literature 
(Chapters Two and Three) that guided this research, namely: knowing-in-action, narrative as socially enabled and constrained social action, the social and historical context of ASL teaching, and the common practices in the profession. I used those codes to "overwrite" the previous codes to attach theoretical significance to the data.

When the codes were somewhat settled, I identified categories that reflected patterns I identified across the codes to "consolidate meaning and explanation" (Grbich, 2007, as cited in Saldaña, 2013, p. 9). Some teachers' narrative units were internally consolidated in cases where the same story was told multiple times or across several tellings. Sample code categories included, for example, experiences in different life stage (e.g., "the early days"), explanations of a particular type of experience (e.g., "teacher training"), and reflections on personal goals (e.g., "teaching for a better world"). I clustered teachers' narrative units around patterns and then re-organized the story clusters around the three chronological eras established by the research questions: pathways to (past), pathways through (present), and pathways forward (future). The clusters of stories that most strongly addressed the research questions became the findings chapters (Chapters Six to Eight).

Narrative units with single codes that did not fit into any categories were put to the side and retained for future reference. For narrative units with multiple codes I prioritized temporal codes to align with the presentation of findings that would be organized according to the research questions and their built-in chronology (pathways to, through, and forward). By the end of the categorization process, I arranged all of the categories according to this chronology to allow teachers' stories to unfold over time. The 
most salient categories appear in this dissertation as subheadings for the story clusters in Chapters Six to Eight (when no verbatim quote applied).

The final step in data analysis was to identify overarching patterns across the codes and categories that spoke to the meaning of the stories at a conceptual or theoretical level (Saldaña, 2013). Themes is the methodological term for these overarching patterns, but I refer to them throughout the dissertation as threads, which is more evocative of storytelling language (e.g., "the thread of the conversation"). In this stage, I returned to the history and background of ASL teaching (described in Chapter Two), the guiding theoretical and empirical literature (Chapter Three), and the data collected in the Mapping phase, described below, which provided broader context for the teacher-participants' stories.

\section{Mapping Phase}

The Mapping phase supported the Storying phase by shedding light on features of the sociological context of the stories (Gubrium \& Holstein, 2008, 2009). The focus and contribution of the Mapping phase changed significantly from planning to inception. Initially, I had anticipated significant input from the SLIC representatives for this dissertation that I was unable to obtain as a result of program politics partway through our collaboration. This shift meant I no longer had access to insider information about the organization and activities of the SLIC, nor to their networks of teachers and other stakeholders. Nonetheless, I had three main Mapping data sources: (1) interviews with SLIC and deaf cultural organization representatives, (2) interviews with ASL program administrators, and (3) publicly available artifacts. Although the data did not directly 
contribute to addressing my research questions, it was useful in contextualizing the Storying findings.

The SLIC and deaf cultural organizations in Canada, I knew from my study (Doré, 2015) and anecdotally, were largely if not entirely responsible for formal sign language teacher training and act as hubs for teacher communication. I contacted the SLIC executive board and six provincial deaf cultural organization representatives via publicly available email addresses to participate in interviews. My goal was to better understand not only what opportunities these organizations offered (and to whom they were made available), but also the history and structure of the organization and its relationship to ASL teaching.

One representative from SLIC and two provincial deaf cultural organization representatives, one current and one recent, participated in one 30-45-minute semistructured interview each. Interview questions targeted the organization's activities, membership, leadership structure, aims, and current and future initiatives. Interviews took place via (recorded) online video chat in ASL. I translated and transcribed all interviews into written English as per the process described above in Storying. I coded the transcripts using basic descriptive codes that identified the main topics of the interviews (Saldaña, 2013; e.g., "teacher training”, "promoting ASL”). I also highlighted salient quotes that reflected key features of ASL teachers' professional landscapes.

Both provincial representatives were ASL teachers who consented to also participate as teachers (Donna and Erica). It later came out that two already-recruited teacher participants were current or former leaders of local deaf organizations (Ken and Nick); they consented to an additional interview as organization representatives, which 
my ethics clearance allowed. These four "double-duty" participants were particularly insightful informants, as they were or had been intimately involved with projects that brought together a number of teachers, e.g., workshops for teachers, and also had a strong community-oriented vision beyond their individual professional goals.

I also reached out to two post-secondary ASL program supervisors via publicly available email addresses, one who was currently a supervisor and one who had recently left their position. Both agreed to participate in one 30-45-minute semi-structured interview each. The questions included, for example, questions about the usual processes for hiring ASL teachers, who was involved, and what kinds of documents facilitated the process (see Appendix I). Interviews with program directors and were also coded descriptively. One administrator provided me with a number of additional documents related to the line of questioning, including, for example, an old ASL teacher hiring committee interview guide. While it was quickly apparent that supervisors' insights about teachers' daily lives were too high-level for the purposes of this dissertation and further recruitment was canceled, they nonetheless helped me to initially situate ASL teachers within the institutions of which they are a part. No further interviews were requested. In addition, I collected publicly available online textual artifacts related to (1) deaf cultural organizations, e.g., bylaws, AGM minutes, statements of board members, flyers for events; (2) institutions that offered ASL, e.g., public course outlines, program descriptions; and (3) ASL teaching more broadly, e.g., news stories about the thenpending (now confirmed) official recognition of sign languages in Canada. Artifacts were compiled into digital folders (or bookmarked in the case of websites) and tagged with descriptive memos. 
As I brought together the codes and memos from the Mapping phase, it became clear that they did not respond to my research questions, and so they served primarily to support my interpretations of the Storying data by contextualizing the threads from the teacher-participants' stories. Insights from Mapping are dotted throughout my commentary in the findings chapters.

To support the claims I made in this dissertation based on the results of the Storying and Mapping phases, I conducted several validity checks, which I describe in the next section.

\section{Validity Checks}

In assessing the quality of narrative work, scholars have looked to general criteria for qualitative research such as validity and credibility (Creswell \& Miller, 2000) as well as narrative inquiry-specific criteria like narrative resonance and narrative interlappings (Connelly \& Clandinin, 1990). As a deeply qualitative methodology, narrative inquiry does not aim for historical truth but rather narrative truth, where "storied texts serve as evidence for personal meaning, not for the factual occurrence of the events reported in the stories" (Polkinghorne, 2016, p. 479). The narrative researcher is responsible, then, for providing the reader with sufficient evidence for claims about the meaning of participants' experiences, how those claims were derived, and the limits of those claims (Polkinghorne, 2016).

Throughout this study I attended to practical, ethical, and methodological questions about such areas as narrative voice, ownership, truth, and authority. Would my transcription protocol be effective in translating the richness of teachers' signed conversations into written English? Did I blur enough details of teachers' stories to 
protect their anonymity? What biases did I carry throughout the work consciously and unconsciously? Were my portrayals of the teachers and their storied experiences fair, insightful, useful, and academically viable? Throughout this chapter I have attempted to be transparent about my decisions in response to these questions. While the achievement of presenting a complete and accurate "truth" of teachers' lives was never the aim (Connelly \& Clandinin, 1990), I sought to enhance the validity of this research in several ways, including members' checks, inter-coder reliability, and thick description (Geertz, 1973). Each of these is described below.

Members' checks have long been considered 'the most crucial technique for establishing credibility" in qualitative research (Lincoln \& Guba, 1985, p. 314), despite their being often poorly defined (Creswell \& Miller, 2000). Members' checks can be carried out in a number of ways, including inviting participants to review or correct their interview transcripts, seeking participants' input on preliminary findings, or sending participants drafts of the final report (Thomas, 2017). In my case, I considered members' checks important to ensure I was "representing the experiences of participants in ways they can recognize and that reflect their realities" (Thomas, 2017, p. 28). This was essential because "[ $t]$ he qualitative paradigm assumes that reality is socially constructed and it is what participants perceive it to be. This lens suggests the importance of checking how accurately participants' realities have been represented in the final account" (Creswell \& Miller, 2000, p. 125). Participants' feedback also gave me the opportunity for self-reflexivity by inviting alternative interpretations that might have highlighted biases I was not aware of bringing to the work (Thomas, 2017). My reflections in Chapter 
Ten helped to articulate my biases and some of the challenges and tensions in my interpretation of this research.

I conducted several different types of members' checks at different points throughout the study with the teachers who participated in this study. First, during the interviews I repeatedly "played back" elements of the teachers' stories (Lincoln \& Guba, 1985) about elements of our conversations to ensure my understanding was on track. I also sent the initial interview transcripts to participants who were interviewed in ASL to verify the accuracy of my ASL-to-English interpretations (there were no significant corrections). These processes were a way of establishing "transactional validity", which Cho and Trent (2006) define as

an interactive process between the researcher, the researched, and the collected data that is aimed at achieving a relatively higher level of accuracy and consensus by means of revisiting facts, feelings, experiences, and values or beliefs collected and interpreted. (p. 321)

In other words, transactional validity is the process of clarifying misunderstandings between participants and the researcher and engaging participants in "making sure their realities correspond with the interpretations brought forth by the researchers" (Cho \& Trent, 2006, p. 322)

After this dissertation was fully drafted, I checked in with three of the teacherparticipants (Nathalie, Michelle, David) about their views on the key themes I had identified from the study. Our check-ins were conversational and largely unstructured. For each teacher, I explained the key findings with examples of how the teachers' responses had informed it. I asked whether they saw themselves reflected in the findings, 
if they were surprised by them or had anything to add, and if they thought I might have missed anything. Nathalie and Michelle were particularly curious about how their stories compared to other teachers, while David was keen on clarifying his stance and aims. I took notes of their comments during our conversation and used them to clarify and enrichen my interpretations throughout the findings chapters.

Another kind of member's check I performed was through informal conversations I had throughout the study with ASL teacher friends and colleagues who were familiar with ASL teaching but not involved in the study. These additional informants were helpful in providing comparable accounts of their experiences as ASL teachers and flagging elements of the study's teacher-participants' stories and my interpretations that may need closer attention.

Beyond members' checks, I sought confirmation of the validity of my narrative coding process through an inter-coder reliability check. I asked a researcher to independently code a section of one of the teacher-participants' transcripts, and then we met to discuss and compare the results of our analysis. A comparison of our micro level independent codes was 43 of 49 codes in common, or $87.8 \%$ agreement. In terms of overall coding, our agreement in discussion was $100 \%$. This check provided further evidence that my codes were viable. A sample of our coding was included in Appendix J.

Finally, I sought to further enhance the validity of this work by providing "thick" descriptions (Geertz, 1973) of teachers' stories in response to the research questions. As Creswell and Miller (2000) explained:

The purpose of a thick description is that it creates verisimilitude, statements that produce for the readers the feeling that they have experienced, or could 
experience, the events being described in a study. Thus, credibility is established through the lens of readers who read a narrative account and are transported into a setting or situation. (p. 128-129)

Daiute and Lightfoot (2004) agreed, "The [narrative] unit of analysis is often big gulps of text—entire stories" (as cited in Saldaña, 2013, p. 134). Presenting large segments of the teacher-participants' verbatim descriptions also helped to keep their voices at the fore, as was my intention.

In assembling the findings and discussion of this dissertation I made a number of decisions about content, sequence, presentation, and organization that will be helpful to explain to the reader. Some of the decisions were intuitive, some of them were guided by academic conventions (including adhering to APA $6^{\text {th }}$ edition guidelines), and some of them were driven by my own sense of ethical responsibility to the stakeholders in this study. These decisions are described below.

\section{Presentation of Findings}

I organized the findings of this study into one chapter that introduced the teacherparticipants in this study (Chapter Five) and three chapters of their stories (Chapter Six to Eight), each of which responded to one of the research questions. I also included a follow-up interpretive chapter (Chapter Nine) to make explicit the connections between the storied findings and the theory, literature, and aims that drove this dissertation. This latter form of chapter is not always included in narrative studies:

The produced finding is held out as the stories themselves. The stories alone are revealing enough to provide insight into the variety of lived experiences among 
the participants. The validity of the story is attested to by its rich detail and revealing descriptions. (Polkinghorne, 2016, p. 482-483).

However, since my intended contributions with this research included contributing to academic conversations on a theoretical as well as empirical level, I decided the interpretive chapter would help to consolidate and make more explicit the scholarly claims of this work.

The findings chapters begin with the following chapter, Chapter Five, that comprised brief narrative portraits of the seven participating teachers. The portraits were written from my perspective as a researcher and the teachers' interlocutor during our conversations. Of course, the portraits are abbreviations and simplifications of rich and complex lives; still, they introduce the reader to the teachers so that the teachers' stories that follow in Chapters Six through Eight can be read with some understanding of who the storyteller is.

Chapters Six to Eight include the core findings of this dissertation: the seven teachers' stories. These three chapters mirror the structure of the research questions and the interviews: background and early experiences (pathways to, Chapter Six), ongoing experiences (pathways through, Chapters Seven), and reflections (pathways forward, Chapter Eight). Looking more broadly, I opted to present and discuss the teachers' stories along this timeline in order to highlight a narrative unfolding of teachers' professional lives over time. Although in these chapters I speak to patterns across different teachers' stories, at no point do I imply that any stories are representative of other teachers'.

To emphasize the richness of teachers' stories in Chapters Six to Eight without overburdening the reader, I discuss the experiences of every teacher in every chapter, but 
in varying depth. The teacher-participants in this study varied in their level of reflection, depth, and detail. Stories that were told in more depth and displayed rich storytelling qualities — for instance, abundant detail, eloquence, and meaningfulness to the teller-are presented verbatim in full or condensed form. In the case of Michelle, however, for whom only our first interview was recorded and transcribed, her stories in Chapters Seven and Eight are consistently narrated by me based on field notes.

Chapters Six through Eight each begin with a general commentary to situate the chapter in relation to the others and a summary of the chapter's story threads that begin to respond to the research questions. I weave academic discussion of the stories throughout all four chapters to some extent and at the end of each chapter, as relevant, but a more developed interpretation in relation to the intended contributions of this study is reserved for Chapter Nine, after all of the stories have unfolded and the reader has the full scope of the teachers' experiences. In making these decisions, my goal was to balance the task of flagging notable discussion points for the reader at the time they appeared in the stories while minimizing interruptions to the stories to give them space to breathe and be read without the constant imposition of an academic frame.

On a practical note, I indented all stories on both margins and retained a slightly reduced line spacing (1.5 instead of 2). This was a slight deviation from the conventions of APA $6^{\text {th }}$ edition, but since the bulk of Chapters Six to Eight were teacher-participants' verbatim stories, I found that the double indentation helped to visually flag changes in speakers and thus better distinguish the participants' voices from my own. Capitalized words in stories indicated ASL gloss (see Glossary), where at times a sign or expression was better captured through an ASL gloss than an English translation. Ellipses within 
stories reflected the speaker's pauses during storytelling, except when used after a period, which indicated that I omitted certain elements of the story for conciseness. Ellipses between paragraphs indicated when the stories presented were not contiguous in the interviews but rather stitched together by me for narrative continuity. In all cases, my aim was to preserve the core meaning of the story while also respecting the guidelines of succinctness and relevance in academic writing.

Having explained the methods of this study and the organization of the upcoming chapters, I now turn to the findings chapters, beginning with narrative portraits of the study's seven participating ASL teachers in Chapter Five. 


\section{Chapter Five: Narrative Portraits}

This chapter marks the first of four findings chapters. In this chapter, Chapter Five, I present the reader with a collection of brief narrative portraits of the seven participating teachers, from their childhood to present day and, for some, their goals for the future. The portraits were written from my perspective with only the occasional verbatim quote from the teachers themselves; they were thus my impressions of the teachers' lives based on what they shared in interviews rather than their own selfinterpretation. The portraits were intended to prepare the reader for the story-based chapters that follow, to help them to attach those stories to their unique storytellers. I do not conclude this chapter with a discussion, as the details of lives are revisited in storied segments over the following three chapters (Chapters Six to Eight).

\section{Nathalie}

My first interview with Nathalie was one early spring afternoon after she had finished her full-time government job in Central Canada. She had run from her office and joined our meeting in jogging clothes, out of breath and bright-eyed. I had met Nathalie several years prior and knew to anticipate an energetic, intelligent, articulate, and friendly young woman. I also knew she was hard-of-hearing, wore hearing aids (since grade three, she told me), and grew up oral, speech reading and speaking fluently. From our first conversation that first day, we slipped, spontaneously, into spoken English overlayed with signs. When I asked which language she preferred for our interview, she opted for English and audio recording only. In revisiting my recordings, our conversations were interspersed with pauses, claps, other signing sounds, and half-whispered words as we found our rhythm of sign-speaking. 
At the very beginning of our first interview, it came up that Nathalie was wrestling with a great deal of instability in her personal and professional life. She had recently learned that her hearing had deteriorated significantly and she was now fast approaching a decision point: get a cochlear implant and continue as she had been in her relationships and job with the government—-her ASL teaching was part-time- or leave the hearing world behind and embrace life as a deaf person. ASL teaching was not just a side gig for her; it was a potential career change should she need to turn her life as she knew it upside-down.

Lately, she had begun to spend more time socializing with the deaf community, partly as a relief from the demands of lip-reading and speaking with hearing people, and partly as a way of building a new community for herself. There was a sense of loss and unrest that permeated all of our conversations, a need for a clear way forward, where ASL teaching played a significant and somewhat symbolic part.

At the time of our interviews Nathalie was teaching a beginner-level ASL course in the evening in the same university program where she had been a student just a few years earlier. It was her second term of teaching; the first term having been one or two years prior. This time, she had been called to teach less than a week before the start of classes. She claimed the late notice did not cause her stress because she had a background in language teaching from having completed a Certificate in Teaching English as a Second Language (CTESL). She was also an alum of the ASL program where she was teaching, and so knew the structure of the program as well as several of its teachers.

Over the course of our interviews, I learned that outside of consultations with her fellow teachers in the program, Nathalie had little contact with other ASL teachers. She 
had not attended any workshops and had no immediate plans to do so. Instead, she looked to her training during her CTESL and to her experiences as a student in the program to guide her decision-making.

\section{Ken}

Ken was born deaf to hearing parents in Eastern Canada and learned Maritime Sign Language (MSL) from his deaf older brother. He moved between mainstreaming (i.e., hearing schools) and deaf institutes for all of his schooling. He said his English reading and writing were "lousy" but his signing was "strong". He was proud of MSL, which he argued was not a dead language like people said. Ken had been deeply involved with a number of deaf cultural organizations over the years, through which he had participated in a number of projects, including several MSL revitalization initiatives, improvements to hearing people's awareness of deaf culture and sign languages, and improvements to interpreter education in the Maritimes.

Ken was first introduced to ASL teaching in the early 1980s by a hearing friend who was teaching ASL classes at a community centre and felt it was not right that he was teaching; a deaf person should take over. This friend (later, partner) offered to mentor Ken until he was ready to take over the classes. After some initial hiccups, Ken found he enjoyed the work immensely and he soon took over his friend's classes, which launched his decades-long career as an ASL teacher.

Throughout his career, he attended many workshops and presentations about ASL linguistics and pedagogy and led several teachers' groups and training sessions of his own. He taught ASL at interpreter colleges on the West coast as well as back in the Maritimes. Apart from a brief hiatus to pursue a lifelong but ultimately short-lived career 
as a bank teller, Ken did not stop teaching ASL. At the time of our interviews, he was teaching private courses to interpreters and other private clients.

Although he had no formal higher education related to language teaching, Ken was very active in professional development activities over the years. During one of our interviews he sent me a list several pages long of the various workshops, presentations, and training programs that he had attended; equally long was the list of those he had offered. He also worked with a group of local deaf teachers to develop an MSL curriculum for interpreters, whom he found to be ill-prepared for work with elderly deaf Maritimers who used a blend of MSL and ASL. His efforts were driven by his belief that Maritime interpreter education needed to be improved through increasing the standards and rigour of preparation programs.

In his years of efforts to this end, he had run into some opposition from other interpreter educators. He felt many of them were too laissez-faire about their teaching and had low expectations for students, which was lowering the quality of interpretation services for deaf people across the Maritimes. Ongoing disagreements as well as secrecy, duplicity, and cliquey behaviour amongst ASL teachers were motivating factors in Ken's decision to leave post-secondary teaching and pursue self-employment and private lessons.

\section{David}

David was the only hearing teacher-participant in this study. He did not grow up around ASL or deaf people and had only started signing during his undergraduate degree after seeing a presentation about sign languages by a deaf ASL instructor. The presentation led him to immediately switch into ASL and language studies. He described 
learning ASL as a "spiritual" experience that helped him to open up and grow as a person.

At the time of our interviews, David had only a couple of teaching contracts under his belt. Like Nathalie, he was hired in the same Western Canada ASL program where he was formerly a student. He turned to his former teachers, now colleagues, to guide his teaching to ensure he was preparing his beginner-level learners for the more advanced, more demanding courses. His duty, he said, was to teach his students how be ASL students in the program. His students' duty, meanwhile, was to develop an awareness and sense of responsibility about what it means to learn and know ASL as a hearing person. Although he mostly taught beginner-level learners, he was adamant that they learn to recognize their hearing privilege and develop a sense of allyship with deaf people.

\section{Michelle}

Michelle and I had met several years prior to this study through an online ASL group. I knew she had been teaching for several decades and was very enthusiastic about all things related to ASL teaching and learning. She had a contagious, sharp-witted sense of humour and could spin a pun as easily in English as in ASL. In our interviews she moved fluidly between speaking and signing — her lip-reading and pronunciation were excellent, having been raised oral with hearing aids. Her English still carried traces of her Midwestern USA accent where she had lived for many years.

She did not come across ASL until she started dating a deaf classmate while studying illustration in college. ASL was still referred to as Ameslan at that time, she reminded me. She started taking ASL courses at the local hearing society and attending deaf events, such as a monthly captioned movie night. She described her early signing 
skills as terrible, "awful", but she persisted and improved greatly with the support of a helpful local signing community. She studied for a brief time at the National Technical Institute for the Deaf, which was a culture shock, as she was surrounded by deaf people and ASL on all sides for the first time in her life.

After graduation, she worked as a graphic designer for six-odd years, and then got married and moved to Canada. In Canada, she turned her attention to ASL teaching. She found an ASL teacher training course in Central Canada and started working as an assistant teacher in a local community centre under one of the teacher training course's instructors. The instructor mentored Michelle until she was ready to take over her classes, which she did. Michelle had been teaching in community continuing education programs ever since.

Our interviews mostly centred on the lesson plans Michelle had spent years developing. She brought out four huge binders, each one containing an extensive collection of activities and learning resources. She was working on a fifth binder for advanced learners. Her goal was to put it all together and publish it as a textbook-"If only I had the time", she kept saying. The lesson plans were a collection of her own ideas and ideas borrowed from books, online resources, and other teachers, although she claimed to have little contact with other teachers because she tended to work alone or in small programs. Occasionally, she walked me through an activity, many of which involved props, costumes, games, and — my favourite — magic tricks. In our final interview, Michelle dug out her large collection of mementos from her students over the years - thank-you letters, teaching evaluations, class photos, and photos of various gifts 
that had been given to her, including many bouquets of flowers. She had obviously made a big impact on many students.

\section{Erica}

Born deaf, Erica briefly attended an oral education program before switching into a SEE (Signed Exact English) program where she stayed until the end of high school. After graduation, she worked at a floral shop with two deaf women who taught her ASL. She found ASL fascinating and soon began socializing with the deaf community. Eventually, she was approached by a local ASL teacher to be a classroom assistant; like Ken, her teaching career took off from there. She had taught ASL in a number of different contexts across the Prairies and Western Canada, including a deaf-blind intervenor program and several colleges. It was not uncommon for her to work several jobs at once, even across provincial borders, all of them related to ASL teaching and deaf services.

Despite having a busy schedule, Erica was active in professional development activities. She was working toward her ASLICE and between our second and third interviews she attended a five-day sign language teacher training course run by the CCSD. She saw these formal credentials as a strategic move because they would give her leverage and credibility with the provincial school board, where she hoped to get hired in order to advocate for stricter qualification requirements for professionals working with deaf children, many of whom she said were hearing and unqualified, with poor signing skills.

Erica was one of my "multi-tasking” participants, as she was both a teacher and involved in her provincial deaf cultural organization. Some of her work with the 
organization was supporting ASL instruction, though most of it was advocacy, as she said her province had a very weak infrastructure for deaf support services, particularly around sign language education for children. She spent a lot of her time in an advocacy role as well as working with deaf youth to foster awareness and pride in their deaf culture.

\section{Donna}

Recently (mostly) retired, Donna had been a leader in ASL teaching for many years and very active in her local and provincial deaf cultural organizations in Western Canada. She was born deaf and attended different deaf institutes up until she finished high school. After graduation, she moved to the USA to do a bachelor's degree in mathematics at Gallaudet University and later a Master's in deaf education. During her Master's she was approached to teach an ASL class for hearing students where she was handed Lou Fant's American Sign Language Phrase Book and told to "take it away". She remembered being completely lost in that first class, with no experience and no guidance, but took the job because she needed the money. She eventually found a rhythm and a love for ASL teaching.

After her Master's, she came back to Canada and accepted a math teaching position at a deaf school. Not long after, a local college established an ASL program for hearing adults and Donna took on teaching ASL in the evening. After nearly twenty years of that — teaching math by day and ASL by night—she left deaf education to teach ASL full-time in various hearing high schools, colleges, and universities. For a time, she was part of a team of teachers that developed a multi-level high school ASL curriculum for the province, as there was not a standardized provincial curriculum at the time. 
Through it all, Donna was heavily involved with her provincial deaf cultural association. Unlike most other provincial associations, hers had been highly successful in securing funds to support a wide range of ASL-related activities, including a robust ASL teacher professional development program—workshops, guest speakers, training programmes, etc. She was among the first teachers to receive the ASL instructor's certificate (ASLICE) through the SLIC. Retired from her other jobs by the time of our interviews, Donna still taught ASL part-time at a local university because she "loved teaching university students". She had been asked repeatedly to take on more courses, but she kept refusing, as she wanted to enjoy the peace and quiet and free time of retirement. Nick

Nick was the newest teacher in the study, having started teaching just weeks before our first interview. Born deaf to a hearing family, Nick was raised oral but declared himself proudly "stone deaf". As he put it in our interview, "Take my hearing aids off and I'm stone deaf". He also had had a deaf "tribal" tattoo to symbolize the strength of his community.

Nick wore hearing aids and his English lip-reading and speech were excellent (at one point he demonstrated an impressive British accent), which he said can cause confusion — and occasionally animosity—amongst his deaf peers who assume he is hardof-hearing. Still, his speech was a point of pride for him and he suggested that his ability to move between hearing and deaf worlds was conducive to his strategy to someday work in politics. He reckoned, "Everyone remembers the deaf guy who can talk".

Nick spent most of his childhood in hearing schools but switched to a deaf school for the last years of high school. Suddenly immersed in a signing environment, he picked 
up ASL quickly and even started teaching ASL to students at the neighbouring hearing school. After graduation, he worked several different jobs, including a few months as an interpreter and educational assistant for deaf children. He enjoyed the job and even applied to a college ASL program to get more formal ASL credentials. After completing only a few courses, he put ASL aside to pursue an unrelated undergraduate degree. Several years later, he took up ASL courses again while completing a second undergraduate degree. He excelled in those courses, leading study groups and mentoring other students. To him, both signing and leadership came naturally.

When we met, he was teaching in the same program in Central Canada where he had graduated as a student seven years earlier. Teaching, he admitted, was somewhat overwhelming. To help him adjust to the new role, he had been consulting with a fellow teacher who has been giving him some guidance and direction. He hoped to make a career out of ASL teaching. He called it "my new drug" since he had recently quit drugs and smoking in the wake of his divorce. He acknowledged that a side benefit of teaching ASL was that it gave him exposure to a wide range of people, potentially future voters, which might help his future role as a politician.

In closing, this chapter introduced the seven ASL teacher-participants whose stories are featured in the three chapters that follow. Chapters Six to Eight dive into the above teachers' stories of experience on their pathways to, through, and forward in their profession. 


\section{Chapter Six: Pathways to Teaching}

This and the following two chapters are a collection of this study's ASL teacherparticipants' stories of professional experience and reflection. This chapter, Chapter Six, attends to teachers' early career experiences, or their pathways to the profession. Chapter Seven focuses on teachers' ongoing career experiences, or their pathways through the profession, with an emphasis on teaching practices and professional relationships. Chapter Eight is about teachers' reflections on their practice and their visions for the future, or their pathways forward.

As much as possible, although some are lengthy, I present stories largely uninterrupted to foreground the teachers' own voices and encourage the reader to connect with them in their own way. I close each chapter with a brief discussion of the main patterns or threads of the chapter in relation to the research questions and the theory and literature that informed it (Chapters Two and Three). I reserve a theoretical discussion for Chapter Nine, where I bring together the main threads from Chapters Six to Eight and consider them holistically in light of the aims of this dissertation.

I used the same interview guide for all of the teacher-participants, but each teacher took our conversations in a slightly different direction. There were expected differences in what they told, such as differences in upbringing, and insightful differences in how they told them and how they positioned me as their interlocutor. There were also common threads throughout stories, such as the importance of mentorship and supportive peers in teachers' early days on the job and the recurrent narrative of DEAF CAN or deaf empowerment through the work of ASL teaching. I clustered the stories into three categories corresponding roughly to three chronological periods in teachers' lives: (1) 
coming to ASL and the deaf community about teachers' background experiences with ASL and the deaf community (2) coming to ASL teaching about the people, events, and circumstances that led to their entry into the profession, and (3) the early days about teachers' earliest teaching memories.

\section{Coming to ASL and the Deaf Community}

Although this dissertation is about teachers' professional lives, as previously explained in Chapter Three, teachers' professional lives were influenced by their prior life experiences with the language and language community — in this case, ASL and the deaf community. These experiences, referred to here as teachers' language lives (Ellis, 2018), inform teachers' pathways to and through their work. The stories in this section highlight the diversity of this study's teacher-participants' pathways to ASL and the deaf community and the diversity of influences it had on their work.

Most of the teachers in this study arrived to ASL later in life. Of the seven teachers, only Donna learned ASL as a first language, having been born deaf and attending a deaf institute where she was educated in ASL. Ken's first language was Maritime Sign Language (MSL) and he learned ASL later in his youth. Erica used Signed English prior to learning ASL. Nathalie, Michelle, and Nick, all deaf/hard-of-hearing, grew up fully oral before learning ASL as teenagers and young adults. David, the only hearing teacher, learned ASL as an additional language in his mid-twenties.

Ken and Donna spent little time reflecting on their ASL learning experiences, perhaps because their signing background reflects the core of the "traditional" deaf experience: born deaf, attended a deaf institute, and use sign language as their primary means of communication. Erica also dwelled only briefly on her arrival to ASL. She was 
around twenty and working in a flower shop with two deaf women when she realized that her Signed English and ASL were distinct languages:

I realized I couldn't quite communicate with them and asked what the different signs meant. They told me that they were using ASL and from there I became fascinated with the language. I must have been around 19 years old.

Erica's coworkers introduced her not only to ASL but also to the deaf community where she became an active member and activist.

Nick, meanwhile, grew up oral deaf in a hearing family and his first introduction to ASL was when he transferred to a deaf school for his final years of high school. He spoke little of his experience transitioning to a signed environment, only that ASL came easily to him:

I only started learning ASL in high school. Yeah. Being deaf, I picked it up really quickly. I also had tons of exposure - I was with deaf people all day, every day! I was waking up, eating breakfast, going to classes, all that with deaf people. I picked up sign pretty quickly. Everything was deaf there. It was a whole deaf world...

He attributed his rapid acquisition to his deafness and his full immersion setting. His new signing environment was the impetus for his beginning to spend more time in a signing environment with deaf people and eventually aim for a career as a deaf educator.

Michelle, Nathalie, and David shared more extensive memories and reflections about coming to learn ASL. For all of them, it was a significant experience that 
profoundly impacted their lives at a personal level, as described over the sections that follow.

\section{Michelle: "I finally met a deaf person!"}

Michelle opened our interview with a humorous narrative about how her parents confirmed her hearing loss when she was a young child:

As a kid when I was in school I went to get my hearing tested. I sat there in a little booth with earphones on my head and I was supposed to shut my eyes and - obviously I kept peeking-I was supposed to close my eyes and raise my hand when I heard something. But since I was peeking I knew when to raise my hand. Peek, oh, raise hand... I managed to do it at all the right times. I think a few times they caught me. No no no, they said, keep your eyes closed.

Eventually the man, the audiologist, he figured out that my hearing wasn't quite right. He gave me a note and pinned it to my coat and sent me home. I got home feeling very pleased with myself, thinking I'd passed this little test. I showed my parents the note-no, it wasn't both parents, it was my father who read it first. He read it, gave me a look, and then he rushed off and brought it to my mother.

I remember they were looking at this note- I wasn't sure what was going on but I could tell they weren't happy about it. I didn't know what the paper was, it was just some paper on my coat. My mom was looking at it and back at me and then she started crying and crying... just bawling... ... [Chuckles] I remember thinking, what's wrong? What's wrong? And my dad said, it's okay, go to my room. 
She described her parents' sadness and her own innocent confusion when she was declared hard of hearing and given hearing aids. In the end, she did not recall having many issues with her hearing, as she grew to be very successful at speaking and lipreading.

When describing her oral upbringing, Michelle reminded me, "At that time AGB [Alexander Graham Bell] was the rage and so sign language was absolutely forbidden at the time". For context, Alexander Graham Bell, or $A G B$, exists in the deaf vernacular as having "contributed significantly to social and economic restrictions experienced by deaf people, not only in his day but also extending to the present" (Mitchell, 1971, p. 356). Bell, who had both a deaf mother and a deaf wife, provided significant financial support for oral methods of education of the deaf, as well as advocated for eugenicist policies against the intermarriage of the deaf. Thus, the legacy of AGB in the deaf community is one of utmost discrimination and enduring harm. By referencing AGB as she did, Michelle accomplished several social actions. She demonstrated her (current) insider knowledge of deaf history and deaf community values that oppose Bell's enduring villain-like status amongst deaf people, and her alignment with them. She also did not pause to explain the significance of AGB as she relayed her story, which suggests she identified me as sharing in the same knowledge of the social and political history of the deaf community and, most likely, the same stance about AGB and oralism.

Growing up in the hearing world, Michelle came to know about AGB and other deaf history and culture after several years of learning ASL and socializing with her local deaf communities. Michelle was first introduced to ASL in college by a deaf classmate: 
At the first school where I [studied] there was a deaf man. Right. I sat near him. One day I thought to ask him, do you know sign language? He did. And he asked me if I did. I told him, 'No no... sorry'. He said, Well, do you want to learn? I was like, Oh yes! I do! I want to learn sign language! So I was maybe 20 years old when I started to learn sign language. It was so good. Yes. I was 20 years old when I first saw sign language and it really struck me. Also, he was the first deaf person that I had met. I was so excited - I finally met a deaf person!

He became my boyfriend actually [Laughs]. Yep. We were sweethearts... He was from... north... somewhere north. Maybe Ohio? Somewhere over there. When we were first dating, he'd come over and my friends would be like, whoa he's deaf! And they just thought it was just the neatest thing. At first, though, I think they probably looked down on him and his signing. Honestly, I probably did a little bit, too. I grew up hearing. I wasn't deafI was special. And sign language and deaf people, well... we looked down on them. They were... lesser. This is back in the time of AGB. That's what I knew, that's what I was used to.

Her story carried excitement but also a gentle apology for her early apprehension and condescension toward her new deaf boyfriend. Michelle referenced AGB to contextualize her early attitudes, where the general sociopolitical climate at the time stigmatized deaf people as lesser, as second-class citizens (Mitchell, 1971). Her and her friends' fascination with her deaf boyfriend were thusly positioned within a point not only in the broader timeline of deaf history, but also her personal timeline on her retrospective journey to identifying as deaf herself. Her emphatic past tense, "That's what I knew, that's what I was used to" reinforces how much she has changed from her early self who at the time was not deaf, but "special". 
She continued to date her classmate who helped teach her some ASL:

We studied together, so that was nice. And he would teach me some sign. Man, it was cool! We'd go to a restaurant, for example, and he'd say- he'd order in sign language. Like, he'd sign 7 - UP, and I would sign 7 - UP. You know, the drink, Seven Up. It was fun. It was really just a little bit at a time.

Michelle's enjoyment of signed puns $(7-U P)$ was no surprise, as she frequently spun puns in our interviews, most of them too visual to describe here. Her recognition here that signing was fun may have influenced her later teaching philosophy (Chapter Seven), where she considers her students' enjoyment a key part of their learning.

Michelle's first experiences of learning ASL (still called "Ameslan” in those days, she reminded me) marked her gradual transition from oral deaf to culturally deaf. Not long after she started learning ASL from her deaf classmate, she took up ASL courses at her local community centre and began attending deaf events. She took one ASL course after another, finding it initially very difficult:

Just getting the ABC's was so frustrating. A... B... C... I got so fed up. [Laughs] Oh my god, it was so frustrating! I just couldn't remember. I couldn't take it all in with my eyes. I couldn't visualize what my hands were supposed to do. That plus lousy receptive skills... I was terrible, awful. But man, I worked at it. Even though I was very frustrated I kept at it. I kept practicing, kept taking classes.

I finished [those] classes and I could still not really express myself. I could spell my name. I could spell pretty well, actually. That was fine. 
As a second language ASL learner myself, I could relate to Michelle's frustration and her growing awareness of how difficult it is initially to feel comfortable in the signed modality for someone accustomed to linear, oral-aural communication. Likewise, in my own ASL learning, I found that spelling and any alphabet-based sign somewhat easier because it was a stepping stone between English and ASL. Interestingly, Michelle expressed more frustration about learning ASL than she did about communicating in spoken English as a hard-of-hearing person (i.e., none), although most likely her emphasis on the former was due to the focus of our conversation, its relative recency and, most likely, its much more significant impact on her current teaching.

Still, Michelle kept practicing. She attributed her tenacity as an early learner to her genuine enjoyment and appreciation of sign language. Later, she also credited her growing social circle of deaf signers who introduced her to new experiences:

Once my signing got a bit better, I started going out and meeting more deaf people. It was very exciting because I didn't know about these people, these deaf people! I was just 21.

I kept meeting deaf people and we became friends. I actually met a lot of people. We'd go out and have a lot of fun together, going out to eat. I remember that we used to go out at night and watch a movie, and this is before CC [closed captioning like today]. We'd set up this big machine that would project the subtitles onto the screen. I thought this was fascinating! ... Every Friday- no, once a month, there was a movie night, a movie night for deaf people. And we would chat, and we would drink... 
But of course I was oral deaf, so I was basically talking and speaking. I was not a very good signer at all. But anyways, they accepted me. It was a nice bunch of people.

I found it was the same in other cities, too. Well, actually ... maybe it was not the same as in other cities... I felt kind of discriminated against when I was in other places, being oral deaf, not so great at signing... But here in my home city it was lovely. It was... I don't know... safer. I knew people, they knew me. We were neighbours... I enjoyed myself a lot.

It was a really good place for me to continue learning to sign, too. It started off really slow and awkward, but it got better eventually.

Michelle emphasized repeatedly how new all of this was to her, how different it all was from her "AGB" upbringing — deaf people ("I didn't know about these people, these deaf people") and subtitled movies ("I thought this was fascinating!"). Unlike when she related her and her friends' reaction to her new deaf boyfriend, this chapter of her life seemed to carry genuine eagerness and openness to becoming immersed in a deaf world.

At the same time, like Nathalie relates later in this chapter, Michelle became aware of the tensions that her oralist upbringing provoked in deaf circles-namely, how central sign language use is to a sense of belonging to deaf communities and how signing was the key to fitting in. Looking at the deaf studies literature, discrimination in the form of audism is certainly a common theme, but it is not often internally focused. Nonetheless, there are documented contexts of horizontal violence within deaf communities. Woll, Kyle, \& Deuchar (1981) described the internal hierarchies in the deaf community, ranking "born deaf with deaf parents" at the top and "late deafened, learned 
English first" at the bottom. Ladd (2005) observed that "statused" deaf members may discriminate against other deaf people in the community (p. 16). Higgins (1980) also noted that some deaf people may participate in deaf community activities, but not be socially accepted as having a deaf identity, such as people like Michelle who were "oral deaf" and speak and lip read.

Clark (2007) claims these situations of internal discrimination are a result of the Deaf Pride movement's need to create a model of a "perfect Deaf person" (p. xviii). The model that stemmed from these efforts is one that now few deaf people actually meet (Fernandes \& Shultz Myers, 2009), leaving many to suffer lower status identity and fewer privileges within their own communities. Fernandes and Shultz Myers (2009) call this being "measured against the Deaf norm" (p. 5), a norm which they argue should be opened up to account for the diversity of experiences of deaf people (e.g., ethnicity, race, gender, sexuality), beyond the White native signer. In any case, if these tensions carried through to Michelle's later teaching life, she did not dwell on them, and the topic of her feelings of exclusion did not come up again.

Nathalie had a less serendipitous path to ASL than Michelle's, but no less impactful on her personal growth, as described below.

\section{Nathalie: "It's something I could have used years ago"}

ASL was initially a "fun hobby" Nathalie began as a child that she did not return to in earnest until her early twenties when curiosity drew her to "officially" start learning ASL in university. Unlike in Michelle's stories, Nathalie's stories of her hearing loss caused disruptions growing up, some of which she described to me. The following is a short condensed account pulled from different interviews: 
When I was a kid, I taught myself some signs just for fun. I thought sign language was cool before it was cool; that's why I wanted to learn it. I never really thought of it as something that I needed. Looking back, I think it's something I could have used ages ago. I missed a lot growing up. I didn't always have that many friends. I missed a lot of tryouts because I didn't hear the announcements. Or when I did try to join a team, I got a lot of, Oh, you can't hear so well? I don't know how well this is going to work.

The same thing happened in university. I played on some sports teams, but mostly coaches would say, You can't do this because it could be a safety issue if you don't hear the whistle or the buzzer or whatever. Honestly, it would have taken a split second for someone to tap me or whatever. Is it going to kill you? What am I even doing here? It was rough. I was a little isolated.

In the above narrative, Nathalie looked back on her childhood and adolescence with some melancholy and anger, recalling her frustration with her peers' lack of perspective and understanding about her hearing loss (“Is it going to kill you?"). Her stories about her social life and interactions changed completely when she told me about spending time with other deaf people:

Things really changed when I "officially" became an ASL student in undergrad. I got really involved with the deaf community right away. I was all confident, with myself, with my signing. I loved hanging out with 
deaf people. Loved it. I loved being a part of the community. I joined the volleyball league, I went out to coffee nights, I did everything. I was going to things like crazy, all the time, multiple times a week, hanging out at deaf events. I went all out. I got a sign name when I was still a first-year student.

When telling me about her past in the first set of stories, Nathalie detailed her experiences of discrimination from her sports team members, coaches, and classmates during her undergraduate studies. The detailed descriptions in her stories were possibly for my benefit as a hearing person who has not experienced the same struggles, and possibly for her own benefit as a form of catharsis. In contrast, she provided few details of her activities with the deaf community (deaf volleyball, coffee nights, etc.), perhaps out of excitement or perhaps taking for granted that I was aware of the implications of them — or perhaps both. Nathalie seemed to assume that I understood the weight of being given a sign name as a first year student, for example, which I could. For the unaware reader, there is deaf cultural etiquette around sign names. Whereas legal names are given at birth, a sign name is given through personal connections in the deaf community. A hearing person who has been recognized and accepted by deaf member of the deaf community receives a sign name that is then as an agreed-upon identifier by others in the signing community. By flagging that she had a sign name when she was "still" first year student, Nathalie emphasized how quicky and deeply she established a presence in the deaf community.

Like Michelle, Nathalie did not seriously take on learning sign language until early adulthood, not because she grew up in the era of AGB, but because her struggles were not with her hearing or language use but with the attitudes of her peers. She 
described herself as "a strong student" who did not feel the need for "accommodations", which she implied is what she may have considered sign language to be when she was a child. As she said earlier, "It's something I could have used ages ago". She described how in hindsight ASL gave her access to a new social life with the deaf community where her hearing status was no longer a barrier, she was no longer in negotiation with peers about her capabilities, and she no longer felt isolated. Learning ASL was a turning point for her.

Also, like Michelle, Nathalie's enthusiasm for ASL was dampened somewhat as she learned more about the social dynamics and internal politics of the deaf community and her own positioning within it. She came back to this point several times throughout our interviews, which I describe in condensed form below:

It was only later I heard that first years going to deaf events wasn't really encouraged. The idea was, you're not skilled enough and you're probably going to piss people off. By then, I was like, well, I'm already going to deaf things so I'm just going to keep on going. But I started feeling that I needed to be more careful about it.

I also started hearing more about deaf politics things and the animosity between hard-of-hearing and deaf people. When I became a teacher, I found out about even more politics, and I was just like, Oooookay, let's be careful. I'm finally starting to get over that. Like, I used to be afraid to tell people in the deaf community that I was teaching ASL. I guess I still feel a little self-conscious about it. Talking to some people, they'll say, 'You're 
not deaf, you're hard of hearing!' I've had to learn to deal with those conversations so that we don't end up yelling at each other.

But the deaf community is like any other community; there are people you're going to get along with and people you aren't going to get along with. It is what it is. People have their own beliefs and you have to let them and not get worked up by it. That's kind of where I'm at. It's a lovehate relationship.

In the first narrative, she linked her first instances of tension-which she referred to as "deaf politics" - to her being a new signer, a "first year". Anecdotally, beginner signers are variously welcomed and dismissed by fluent signers, depending on any number of factors - pre-existing relationships in the community, signing capabilities, general attitude, and so on. She acknowledged that her presence was perhaps contentious and adjusted her behaviour accordingly. In the second narrative, however, Nathalie showed more assertiveness as the tension came from her being hard-of-hearing, an immovable reality. She was also a more established signer and community member by then. By the third narrative, it was apparent that her relationship with the deaf community was a work-in-progress - “I guess I still feel a little self-conscious about it" and "That's kind of where I'm at. It's a love-hate relationship". As explained in Chapter Two, the dominant discourse around ASL teachers is that they will be culturally and biologically deaf. Nathalie, who started ASL "for fun" and has only recently begun to identify as deaf, was aware of this discourse (and recognized that I was too) and that she was perceived by some as violating it. Nathalie's reflections pointed to her gradual understanding and still- 
developing acceptance that the deaf community is wonderful but also real and not without flaws, and perhaps it was not the utopia she had felt it to be in her early learning days.

Overall, Nathalie suggested that in the ten years she had been signing she had built up strategies for navigating conflictual discourses ("You're not deaf, you're hard of hearing!"), but she had not fully found reconciliation ("I'm finally starting to get over that"). These experiences may have informed how she approached teaching her students about deaf culture:

With my students, I feel like there are some things about deaf culture that they're probably better off not knowing. Like the hard of hearing versus deaf identities and how people relate or refuse to relate to each other. There are a lot of touchy subjects. If someone asks, really wants to know, then I'll touch a little on deaf vs hearing, just a little. But they don't need to know about all the drama between the deaf and the, oh you're not deaf enough... If they get involved with the community later on, they'll figure it out for themselves. In first year, they learn about the history of sign language and a bit about deaf culture; that's enough. I don't feel the need to tell them more.

Her stance that students should learn about deaf politics and "drama" on their own through experience may have been a recognition that her students' experiences with the deaf community will likely be unique from her own, as the community is highly diverse, not to be reduced to one ideology or one set of experiences. Or, her stance may have been a matter of respect, loyalty, or kindness toward the many deaf companions who had welcomed and supported her in her own participation in community activities. It is also 
possible that teaching first year students who have neither the life experience nor the signing abilities (Nathalie's classrooms are no-voice) to engage in these conversations has influenced her position.

Unlike Michelle and Nathalie whose stories of coming to ASL and the deaf community referenced their perceptions of being (not) "deaf enough", David was a hearing ASL teacher who framed his experiences learning ASL and beginning to socialize with the deaf community in a different way. I describe his stories next.

\section{David: "It was... spiritual"}

David came to ASL by chance when he was studying Japanese language and culture in university and an ASL instructor came into one of his classes to give an interpreted presentation about ASL. David was "hooked":

[The instructor] came in to do a presentation on American Sign Language. Intro to applied linguistics. First real exposure to American Sign Language. Like, first conscious exposure, I should say. I don't have any recollection of being really exposed. I imagine I saw it in the little bubble on the Parliamentary channel or something like that, but never... connected.

It was an interpreted presentation. So another teacher stood, like, further back and spoke what he was signing. And I was fascinated by it... I... was fascinated by the presentation. And the language itself. So I took intensive first year in the summer and I just continued. By the time I got to my fourth year of ASL I had abandoned Japanese... and I had fully invested myself in research relating to American Sign Language and deaf issues. 
Although he had already spent time studying a foreign language (Japanese), David emphasized the novelty of ASL and his instant attraction to it, both to the differences in communication strategy (interpreted presentation) and mode (signed modality) from the spoken languages he was used to:
ASL is not like any other language. Any- any spoken language. Every spoken language is unique in its own way. Every spoken language has its own beauty, its own poetry. But, in the end, they are all spoken. And that's something I grew up with. I might hear a language I don't understand but I understand that it's spoken. I get that it's a language. It may sound like gibberish but I know it's not.

\begin{abstract}
American Sign Language is contrary to that upbringing. Completely contrary. So. I don't want to use the term exotic because it's so derisive, but the experience of being different in something that appeals to me. And... so, it just fascinated me. It was worth it to take first year and by the end of first year it was a done deal. I was hooked. Yeah. Oh yeah.
\end{abstract}

Here, David referenced a longstanding myth about ASL: that it is not a "real" language (see more myths in Chapter Two). He described how as a hearing person who grew up with no exposure to sign languages, he was initially drawn to sign language because it was "exotic", although he resisted the term to avoid the implied condescension of the term.

Like Nathalie, by his second year, David was attending weekly deaf events, which he saw as essential: 
And you have to. If you wanna get good at it and you wanna understand, you have to go. Because that's where you see the stories. That's how you see the pragmatics, that's how people interact. And you make the mistakes.

By the time we spoke he had completed all four levels of ASL and several courses in linguistics, and so he had significantly more language to describe the ASL learning process. He also had several years of participation in deaf events under his belt, which most likely informed his emphasis on experiential learning through ASL storytelling, pragmatics, and the social aspect of language use.

David's continued ASL studies and participation in deaf community activities proved eye-opening in ways that extended beyond his initial fascination. Specifically, David came to recognize Japanese as closing him off and ASL as opening him up. Although the story below is lengthy, kept together it demonstrates David's eloquent theorizing of his experience:

Culturally, American Sign Language and Japanese are very different. English is sort of in the middle. Japanese is a very circuitous language. Not the language intrinsically itself, although there's a bit of an element of that, but culturally, because of... pressure towards group harmony you don't tend to express yourself directly about issues. That is an issue I had a problem with on a personal level because of my nature. I was drawn to Japanese culture because of the formality.

Once I finished first year American Sign Language I realized that was an unhealthy thing for me. not for people, but for me. It's not that ASL doesn't have the ability to be circuitous and it's not like people don't 
deceive. But it tends to be a very direct and emotive language. It's clear about its emotional content. And that appealed to me. In the sense of the different and the terrifying and the personal growth.

ASL is a very direct language. It's a very flexible language. There are registers. You can be more polite, more formal, all of those things. But they're not presented by huge differences in language use. They're presented by differences in posture, and face and interaction. Personal distance, that sort of thing. And just the ability to openly emote emotions is a drastic difference. Because you're not supposed to be quite so emotive in Japanese culture- You can do it within safe pockets. But that notion of safe pockets is problematic for me because I lived my life in safe pockets and they weren't healthy for me. For me. So. That one was closing me off and this one was opening me up. So I chose the one that I felt was better for me.

It was a challenge. Um... And it was something I needed to do more of because I tended to be a very closed off person. so it was an opportunity to let it out and get better at letting it out. It was... spiritual. Spiritual. Japanese is a highly analytic language. It's a fascinating language, but... the- the people who put it together obviously... either consciously or not, think that working hard at a language is a good thing. Like working hard to use a language is somehow a good thing. Japanese is an incredibly complex language. ASL is not like that. You need to be able to emote your emotions. If you're [gesturing stone-faced] you're not going to fit. You have learned to bring that out. That's a real struggle for some people.

I remember for me, first year, my teacher was like, you're too serious. I'm like, I'm too serious? They're like yeah, you're too serious. And that one I had to be like, Oh, I'm too serious. I'm not that serious a person, I'm actually quite goofy. But what I wasn't was showing it. Right? Partly, 
discomfort, but also partly because I was held back by the conventions of communication. You only show you're goofy with people who allow you to be goofy. The answer here, is that you should be goofy by default and anyone who doesn't want you to be goofy will show it on their face. It's a switch of the assumption. So. That took some time. And doing.

David wrapped up his story by repeating that the type of deep and transformative learning that he experienced comes only with time and firsthand experience outside of his comfort zone. He reiterated how he embraced moments of discomfort on his way out of the "safe pockets" that "weren't healthy" for him. His story is one of personal growth triggered by ASL learning.

In our conversation, David took on the role of teacher in our interview as he explained the finer details of the contrast between learning Japanese and ASL, not taking for granted that I shared in any aspect of it. His monologue-like storytelling gave an "open book" impression of his character and a willingness to express his vulnerability. Interestingly, David dwelled principally on the impact of ASL as a language on his personal growth, and spent hardly any time during our interviews reflecting on the impact of his participation with the deaf community, although he alludes to the importance of it.

A final small note about David's narratives is his alternating between "American Sign Language" and "ASL". Since his interviews were in spoken English I was able to note these fine-grained language choices more easily than with the other teachers. It is possible that David's use of the unabbreviated form was for academic effect, to remind me that he understands that ASL is a language on par with Japanese in terms of "realness". He was perhaps also establishing himself as an ASL user who is aware of and rejects commonly held misconceptions about the legitimacy of ASL (among others, as 
described in Chapter Two). Of course, it may also simply be one of David's discursive idiosyncrasies.

The next cluster of stories are about teachers' entry into the profession; in other words, the experiences that first brought them into the classroom.

\section{Coming to ASL Teaching}

Although all of the teachers in this study expressed a sincere commitment to their work, no one expressed being drawn to ASL teaching prior to starting their first teaching job. The only exception was perhaps Nick who had led ASL study groups and informal tutoring sessions for his classmates in high school and university. Otherwise, all of the teachers had —and in several cases still have—alternate career paths. The diversity amongst teachers' training and backgrounds in the literature (see Chapters Two and Three) is often examined from the perspective of standardization of qualifications and thus diversity is implied to be a deficit to the profession. In this study, however, the diversity is a demonstration of the uniqueness and richness of teachers' experiences, particularly since there is no standardized training program for would-be ASL teachers. Generally, the stories teachers told about their pathways to ASL teaching were not at extensive as the stories about their pathways to ASL and the deaf community, Still, it was enlightening to get a glimpse into their broader professional live narratives, as they were fairly diverse - many of them roundabout, unexpected, and even terrifying.

Ken, for example, always wanted to be a bank teller despite being told repeatedly he would not be able to as a deaf person:

It's funny, growing up my goal was to work as a bank teller. I really wanted to work as a bank teller. My teachers kept telling me no no no no, 
it'll never happen. So I kind of gave up on that and started teaching. And I really loved it. Then I got a[n ASL teaching] contract and loved that.

Ken did end up training and working as a bank teller, pushing back against the audist, disability-oriented DEAF CAN'T narrative he was told by doing it anyway-DEAF CAN. He worked at a bank for a year before returning to ASL teaching. Within that year, he was held at gunpoint twice, which was enough: "I didn't want to work in a bank again. I had had enough of having guns pointed at me." He went back to ASL teaching after that and never looked back.

Michelle, meanwhile, unsuccessfully cycled through several college majors (special education, psychology, physical education, to name a few) because she was unable to hear the lectures and did not have an interpreter at the time (nor did she learn to sign until later). This was discouraging: "When I thought about what I could do there weren't that many options. I mean, a whole bunch of other things - math, science, whatever... They were all a fail because of my hearing." She eventually found an illustration program (where she met her deaf boyfriend) and graduated with a Bachelor's in Fine Arts. Her degree landed her a job in graphic design where she stayed for six years before starting on a new career path as an ASL teacher.

Several other teachers similarly completed post-secondary education and built careers in unrelated fields before or while they started teaching ASL. Donna completed degrees in mathematics and deaf education and spent twenty years teaching deaf children while moonlighting as an ASL teacher for hearing adults in college and university. She recently retired from teaching math but has continued to teach her evening ASL courses. Nathalie trained in several unrelated fields—engineering, humanities, English teaching 
(CTESL) and information studies - and continues to work full-time with the federal government while teaching ASL in the evenings. Nick studied social sciences and public policy in university and taught ASL classes alongside his restaurant work. David was teaching ASL part-time in university while also working part-time as a deaf adult literacy instructor and completing a graduate degree (when we spoke, he was already considering another graduate degree).

Only Nathalie and David came to their first teaching roles in a truly "conventional" way: there was a job posting to teach in their former ASL programs, they applied, interviewed, and were hired. Nathalie described her hiring as abrupt and unexpected, but exciting:

The week before classes started, on a Thursday, they were like, do you want to teach a class? Oh yeah, sure. You start Tuesday. ... Okay! It was pretty much out of the blue! I think I got a message from the coordinator or something saying hey, so yeah... a class is available. I didn't expect it, even though I applied, so I knew I was on the list. But I thought, oh maybe they'll have a class, maybe not...

Then the winter break happened and I heard nothing, so meh. I dropped the idea. I figure there's no classes... Then it was like, oh. Oh, okay! I don't have any time to rehearse or anything. That's fine - I'll go through my old notes and figure something out. Go! [Laughing]

Later in our interviews Nathalie lamented the lack of advance notice because it meant that she was unable to prepare certain activities, such as icebreakers and an initial scan of students' needs to help structure her teaching. Still, and as she reminded me several times 
throughout our interviews, her confidence in her abilities to hit the ground running stemmed from being a prior student of the same program and her language teaching background.

Neither Nathalie nor David explained their hiring process - what was required in the application or interview process, how they prepared, and so on. I turned to the two ASL program administrators that I interviewed to clarify the hiring processes in their respective universities. They helped me to contextualize what Nathalie and David's hiring might have looked like given they both worked in a university program. The administrators both prefaced their explanations by explaining that they were several levels removed from the hiring process and that the decision-making sat almost entirely with the senior teachers in the programs. One of the administrators provided me with a recent interview question guide that had been created by their program's teachers, who also led the interviews. The guide included questions about teaching philosophy, classroom methods, problem-solving scenarios, to name a few. The same administrator explained that the process was more formal now than it once was:

I'm trying to remember. ... There may have been a period early on when [one of the teachers] would recommend me somebody and I didn't do the hiring. One had, maybe it was time pressure? I don't really remember the details. Because early on I feel like I didn't know enough about the program or what kind of qualified people would be out there for it so I relied on him a lot.

He followed up by explaining that while most of the language instructors in the modern languages department at his institution had Master's degrees, it was not the case for ASL. 
He justified: "It was I guess, pretty obvious that there were people lacking in qualifications that are, would be competent teachers anyway." He did not define "qualifications" or "competent", both of which have proven difficult to define in the literature.

The other administrator similarly clarified that while he was involved in the ASL program it was largely in an administrative capacity, with little input on the structure and organization of day-to-day happenings:

I think it's really important for the programs to work in an organic sort of way, that's a bit more bottom-up or grassroots-y, where, you know, they, control their program, they have the vision of their program. And, it seems to have worked.

Given the recurrent expressions of ASL teaching being a site of struggle between hearing and deaf control (primarily in deaf education, but extending into ASL for hearing adults), it was an important detail that both administrators noted that key processes like hiring and curriculum development were or should be (mostly deaf) teacher-driven. Both of the administrators were cautious of the political, ethical, and professional need to leave major decisions to the teachers.

Apart from Nathalie and David, the other teachers mostly came to ASL teaching through friends and acquaintances in the deaf community. After Erica was introduced to ASL and the deaf community by her coworkers at the floral shop, she was approached by a local ASL teacher to be a classroom assistant. She described her initial duties as mostly making copies and handing out materials and supporting students by correcting their 
signs and clarifying instructions from the teacher. When I asked her why the teacher felt the need for an assistant, she explained,

At the time there was a teacher shortage and I was young and eager. They wanted to invest in me and help to develop my skills, which I was happy to do. It was trial by fire and all learning through osmosis, observation, and getting my feet wet. I was naïve and brought on informally without a resume or interview. I was approached and told I would be trained to be an ASL teacher.

Erica's description of her entry into teaching was informative. First, she pointed to her apprenticeship as provoked by a teacher shortage. She later explained that the teacher shortage in her province was ongoing and the practice of approaching deaf youth for informal teaching apprenticeships was still common. No doubt apprenticeships serve multiple functions beyond enculturating new teachers into the practices and discourses of the workplace. For instance, involving youth in ASL teaching can help to build a more robust workforce as well as more robust connections within the deaf community (e.g., McKee \& Woodward, 2014). Providing youth with meaningful employment and mentorship is also a significant opportunity to promote a DEAF CAN mentality.

From a narrative perspective, Erica's reflection on her entry into teaching was brief, simplified. By the time of our interview, she had been teaching for over ten years, so perhaps her brevity was a function of the memory fading over time. Or perhaps it was that the experience was exactly what it was intended to be-a supportive learning environment for a naïve teacher-in-training - and so she did not consider it relevant to describe further. 
Michelle had a similar experience to Erica, starting out as an ASL classroom assistant alongside an experienced teacher. When she left her graphic design job and moved to Canada, Michelle was looking for work and came across a workshop "for deaf people who wanted to become teachers". She signed up and hit it off with one of the facilitators, Suzanne (not her real name), who took her under her wing, and later another teacher (Joan, not her real name) whom Michelle eventually replaced.

After the workshop, the college hired me as a teacher support, which was very exciting. So, I was a teacher support. I started off as an assistant for Suzanne, who was a teacher, and I was basically a teacher in training until I was ready to have my own class.

At first it was a bit awkward to learn the lay of the land, and then... I remember, I think, who was it... Joan... Joan something. A deaf woman. She was also teaching for the school board and she heard they were looking for teachers and so she encouraged me to apply. She said I could replace her, take her job since she was leaving soon. So I did. That was at a [high] school... I taught there for a while and then I taught at another school... and another one.

Michelle mentioned her relationship with Suzanne many times throughout our interviews and expressed deep gratitude for the guidance Suzanne had offered. Michelle also gave me the workbook that she used in the workshop. I revisit the workbook in Chapter Seven.

Like Erica and Michelle, Donna was brought into ASL teaching to help out a friend who was "stuck" needing an extra pair of hands in the classroom. She joined the classroom with "no background, no experience, nothing. I was brand new. But I had 
some time, some motivation." It was not long after she began teaching that the SLIC was established; Donna was among the first to complete the SLIC certificate (ASLICE). Her teaching jobs afterwards all required a formal application and interview process.

\section{Nick: "It's a pretty good match"}

Nick's entry into teaching was a combination of formal and informal processes.

He went through a formal application process to be hired for his position, but his application and early practice were prompted by a teacher-acquaintance from the deaf community:

I got the job here because [the ASL program supervisor] knows that I have a B.A. [Bachelor's degree] and they want you to at least have a B.A. to teach [at the university]. That's the minimum requirement to teach. And that's a really big issue cuz there's lots of deaf teachers who are really skilled, very experienced, all that... but they don't have a B.A. and so they can't get hired here. It's a really strict requirement. There's not really a lot of flexibility. Well, there's flexibility for people who were hired a long time ago.

[The program supervisor] knew me from before from the deaf community. She knew me from, you know, just being around, different things. I'm very involved in the community. So she knew that I graduated university, twice. And she e-mailed me and asked if I was available to do some teaching work and I was like, Yeah, absolutely I'm available! Because my other job- I work at a restaurant, and it's pretty flexible timewise, so I mean it was pretty easy to make both of them work. It's a pretty good match. 
Nick's experience was notable because on the one hand he was hired with little prior teaching experience and no background in language teaching and learning, which is the situation scholars argue to avoid (see Chapters Two and Three). On the other hand, he fulfilled the institutional requirement of having a Bachelor's degree, which as he described made him technically suitably qualified for the position. He later described that he was engaged in a form of informal mentorship with his colleagues: he spent time discussing his courses with the program supervisor and a handful of other teacher to make sure he was aligning with the program aims. The way he told his story, he did not question his hiring or express concern about his Bachelor's degrees being enough to prepare him for the classroom. Elsewhere in his interview, though (as described in the next section), he described his nervousness as taking on the work and his careful attempts to align with the other teachers in his program. All in all, he was aware of the demands of the job, but willing to take them on.

Strictly speaking, Nick did have prior ASL teaching experience. When he moved to the deaf school, Nick started teaching kids from the neighbouring hearing school, for fun:

When I was in high school, there was another school in the same area, a hearing school... and I thought it would be pretty cool to share some sign language with the students there. So I went in and taught mostly just basic stuff, like "Hi, my name is..." and "How are you?" Stuff like that.

I used to teach them pretty much wherever, anywhere. In their residence, in their gym... wherever there was space. We would all sit around in a circle and somebody would hold up a yellow ball and be like, "What's the 
sign for this?" And we'd go around - OK, what's colour is it? Yellow. Yes, good. It's yellow. And the shape? OK, it's a ball...

I would do all of this completely voice off. I mean... I wanted to, but I thought it was important because my speech is pretty much perfect so I thought it would be a little bit confusing if I was voicing and signing and going back and forth... So that's why it was all just silent. I remember wanting to speak but I didn't.

His storytelling suggested he wanted me to be aware that he was not completely without teaching experience. He mostly foregrounded his decision not to speak during lessons. As described in Chapter Two, the no-voice policy is common in ASL classrooms. The most common reason to implement the policy is to respect the teacher-who is implied to be deaf - and to encourage an immersion setting. Yet Nick's concern about using his voice during his tutoring sessions was that it would be confusing for him (moving between signing and speaking can be cognitively and physically demanding) and for his hearing learners (taking in signing while listening to speech can be equally demanding). He did not comment on his stance on speech in his university ASL classroom.

His self-directed teaching efforts continued when took on a volunteer teacher position tutoring his classmates in his university ASL courses:

I started ASL again during my second B.A. When I was in my last year, I did things like... discussion groups- like, conversation groups, you know. It was actually all on my own time. I asked one of the teachers, I said, I really wanna get involved in this program. I said, can I come to all of your classes? So I helped out every class he did. I would have students come to me from different levels in asking me questions and asking for help about 
their papers or whatever. Sometimes they would ask me for advice about how to video themselves for their tests and stuff to practice. How to position the camera, where to face, stuff like that.

To be clear, I wasn't actually formally a classroom assistant or a teacher's assistant or whatever. It was just on my own time.

Nick's main motivation for taking on this role was simple: “I don't like seeing people not do well in sign language". In the end, there were some program politics that brought a halt to his informal tutoring. Still, his efforts demonstrated not only his ability to move between hearing learners and the signing world but also his intuition for teaching and his eagerness to share ASL with the world.

In terms of narrative form, Nick's stories repeatedly cast him the main character even though the topic tends to be others - his fellow learners and their progress, friends, colleagues, and so on. This was a common feature of his stories, which I interpreted as a reflection of his narrative style rather than of the relative importance of others in his professional life.

\section{Ken: "I'll try it out, see if I can do it"}

Ken vividly recalled his first time teaching, which was a nerve-wrecking experience that his hearing mentor (later, partner) helped to make safe and meaningful to Ken's professional development. Ken repeated his story twice in different interviews. The first time he described the experience he emphasized his reactions to being in the classroom; the second time he focused on the influential role of his early students and his mentor in building his confidence and skillset as a teacher. Both times his energy for teaching and his gratitude for his mentor were profound. 
After finishing high school, Ken was looking for work. A hearing friend of his who was teaching ASL at a local community centre convinced him to try teaching:

A friend of mine asked me if I knew how to teach ASL - because I signed MSL. So, I was intrigued. I thought, okay, I'll give it a try. It was a friend of mine, someone who had been teaching a long time. A hearing person. He was teaching sign language. He saw me and he said you should teach sign language. I think you'd be good at it. I took it into consideration. I thought okay I'll try it out see if I can do it.

I went up front in the classroom and the hearing teacher sat down said go ahead. Okay your turn. I started hello my name is... writing on the board. And it was pretty awkward. I was doing my best to explain slowly and the students they were all hearing they didn't understand what I had written on the board. They didn't understand my sentences. They were all confused and it was because my grammar. My syntax is all mixed up. They were hearing and not understanding what I was trying to teach them.

I could see them talking to each other, so my friend, the hearing teacher, pointed out to me that they didn't understand because what I had written was bad English. And then I realized okay... so he started coming over and we started working together and he started helping me with my English and very basic ASL glossing so that I could see the word order in ASL also.

But then I noticed that in class when I would sign something, the students would write it down and they would show me. The writing was in English. The word order is English, and so that's how I really got to learn about teaching. 
Ken was a tremendous storyteller and stories like this are only modestly captured in written form. The above was vividly described. He transported himself to the front of that early classroom where, sweating profusely (physically or metaphorically is irrelevant), he stood next to the chalkboard, his hand shaky as he wrote uncomfortably in English to a confused audience of hearing students with whom he was unsure how to interact. As the story went on, his nerves were calmed, replaced by curiosity as he realized that teaching is a learning process in itself. Indeed, through all of his interviews, Ken held strong views about teaching, but they were always underpinned by an openness and willingness to learn, traits that reappear in Chapter Seven in his reflections on his teaching philosophy.

The second time he told this story, Ken elaborated on his students and mentor's contributions to his early learning and development as a teacher. It is worth starting the story from the beginning, where he gives a more nuanced lens on his learners:

That first teaching gig was in a community center night class. That's where my friend the hearing teacher was teaching. He was a hearing person, very skilled in ASL. He been teaching for a long time but he felt, no, now it's time for deaf people to start teaching.

Up until that point it was all hearing people teaching sign language to all hearing people. So when I got into the classroom the students thought I was hearing too. When they found out I was deaf, they were like, shocked. And scared. And all like, I don't know what to do!! Seriously! They wondered, well how are we going to communicate? They were really concerned. [Laughs] Maybe they thought it was going to be lots of miscommunications. 
Unlike his first narration, this time Ken brought in his students as key players in the story of his first teaching experience. In doing so, he rewrote the story more extensively as one of mutual learning. I have included the entire story here, as I felt it was a powerful example of multiple perspectives on a single experience:

In class my English writing wasn't great and often the students didn't understand. But we had a system where every time I would make a mistake on the board, a student would run up and correct what I had written. There were lots of changes they would make to my English, and I would just let them go ahead and they'd corrected it, sit back down, and then we can all proceed now that all of the students understood. It became a little routine. I would sign something and then write it on the board. A student would come correct me, my English. And so we learned from each other.

I learned also that I had to be more careful with my English when I was teaching. For example, the sentence "I bought a car". I would write "car I bought" and the students would be looking at it going no no no, that's not right. And they'd write "I bought the car". But then of course the problem was they started signing with English word order! No no no no no no no that's where I would correct them. "Car I bought". And the students are going- they didn't understand that I was using ASL. I couldn't explain to them - about nouns, topicalization, verbs... all of these things. I would try to explain it to them but I didn't have the language for it because nobody had taught me these things.

Later, my hearing partner taught me about nouns, verbs... what's what... all of the linguistic terms, terms for facial expression and gesture... and that's how I learned more about the language so I could continue teaching. 
In the same story, he spent more time describing the centrality of his partner's role in his teaching:

In that early class my hearing partner and I taught side-by-side. For example, I would teach something and when the students didn't understand my partner wouldn't tell them or correct me - instead he made sure that the deaf always had the power in the classroom. He sat back and he would observe me and the students and feed me little tidbit of insight if it seems like the students and I were miscommunicating. But he always left me in charge.

The advice he gave was always in private, one-on-one, not putting me on the spot in front of the class because he knew that the students knew he was hearing so he wanted to make sure to respect my space as a teacher.

When I started doing that, let's see, how old was I? [Uses a calculator, laughing] Ha ha wow that I was in my 30s? No no no, that seems wrong... I think that's wrong. Let me figure this out. Yes, I know. I was wrong. I was 21 . Yes, only 21. Brand new, awkward... It was a great opportunity, really...

I was quite interested in language before that also. This hearing man, this teacher, his neighbor was deaf so he learned to sign from them and that's how he became an interpreter. He felt that his teaching ASL wasn't right, that it seemed wrong. Then I met him, and I had been looking for work, I could sign... and that's when he said, oh you can sign, I think you can teach. And he coached me through. He really guided me. He came over a few times to make sure I was doing okay and then he left me on my own. I was a bit panicked. I wasn't totally sure what I was doing. At the beginning especially I was a bit anxious about it. I wasn't sure what to do. 
You know, it was all sweaty face, darting eyes... and then gradually, over time I got used to it and it was really enjoyable.

Ken's story is a reminder that on top of never having been at the front of a classroom, like many deaf people, he had also not had any formal exposure to sign linguistics; as he said earlier, "Nobody had taught me these things". With the support of his students and his mentor, he learned about his own language and about English through mutual vulnerability and respect. Thus, his early exposure to ASL teaching became a time of personal and profession growth and empowerment. His growth continued when he moved to the West coast and his teacher-mentor, now his partner, encouraged him to seek work at the local interpretation college. At first Ken had his doubts about his abilities, but he ended up taking on the challenge and working as an interpreter educator for many years:

I thought oh no no, I'm not ready for that. I know how to sign but I don't know how to interpret. I don't think this would work. I mean, people would think I was crazy cuz I signed [Maritimes] sign, not [West Coast] sign. It would be very confusing, and I didn't think that I could balance the two varieties well enough for interpreters. Well anyways and I started teaching and in the end, I just continued. I haven't stopped since.

After he separated from his partner some years later, he started to attend all kinds of workshops with scholars, educators, deaf figures:

I've gotten used to having people around me who are experts, have written books, have Ph.D.'s... And this is how I've been learning all this time. I've picked up so much from them. I met all of these people through my 
partner. He would interpret for them and put us in touch, so it was really lucky for me that throughout all those years I had these contacts. I'm very, very grateful to [him]. He was a wonderful man.

I pick up on Ken's ongoing professional learning in Chapter Seven. Below, I describe teachers' early experiences once they got into the classroom.

\section{The Early Days}

Ken's above stories about his early days of teaching sprawled across a broad timespan as he simultaneously recalled his first day, his first class, and also the years that followed that were all part of his narrative about his induction into the profession. This current section briefly considers other teachers' reflections on their early growth that followed their entry. Chapter Seven picks up where this section leaves off by looking more in depth at the variety of professional development activities teachers have participated in throughout their ongoing time as teachers. The division between early and ongoing career is blurry and somewhat arbitrary, especially with participants such as Nick, Nathalie, and David who were fairly new teachers. I made no attempt to draw a hard line between professional life stages, as for the purposes of this dissertation time was entirely relative to each teacher's own personal timeline.

Looking across the stories, the teachers who had been students themselves turned first and foremost to their prior experiences as ASL learners and to their former teachers (now colleagues) for guidance. Their priority was to align with other teachers and the philosophy of the program. In contrast, Erica as well as Michelle and Ken turned to formal and informal channels within the deaf community for training, independent of 
their workplaces. Whether Nick, Nathalie, and David's development practices will shift over time is yet to be seen.

\section{Nick, Nathalie, David: From student to teacher}

Three of the teachers in this study - Nick, Nathalie, and David — were in the position of being new to teaching, but not new to their particular teaching environments. All of them were previously students in their respective programs in which they now teach. One of the ASL program administrators conjectured that their ASL program's success was due to instructors at his institution "buying into [the] vision" of the program. His program, unlike the majority of ASL courses that use Signing Naturally or other textbooks and written materials, did not rely on a textbook or written curriculum for teachers to teach from. Alignment with the "vision" was therefore essential to keep teachers on the same page, and the administrator noted that buy-in was strongest when teachers were "home-grown", i.e., former students of the program. He said it was sometimes problematic when outsiders came in to the program:

I don't know, you know, I saw it with [one of the teachers], for example who was not home-grown and he had difficulties, I guess, understanding the program I think but I think just through communication and time and the lack of, you know, there's not a written curriculum so it has to be a lot of hands-on mentorship.

The administrator reiterated the importance of mentorship, which in the case of "homegrown" teachers, started several years before teachers coming from outside of the programs where they were employed. 
Still in the midst of his early teaching days at the time of our interview, Nick reminded me that it was only very recently that he was a student sitting in the same chair as his students, but now he is the one at the front of the class:

It's actually really cool. I mean, I went from being a first-year student to helping the students as an assistant. And now I'm the teacher. It's pretty wild. I've seen these three stages and that's pretty neat. It's a brand-new context for me so I'm feeling a little bit... Like, disoriented.

Overwhelmed. A little bit in shock! But at the same time, I'm really enjoying this.

His reflection showed an awareness that he was still adjusting to his new responsibilities and new identity as a teacher, on top of the practical realities of managing a class. He presented at the same time an uncertain and humbled narrative of the new, floundering teacher, and a person who is invested and ready to take on a challenge.

Nathalie similarly made a connection between her not-so-long-ago student life and her current teaching role. She pointed to her time as a student and the one term she had taught nearly two years prior as her main sources of insight, but she expressed concern that her understanding of the ASL program's philosophy was perhaps out of date:

I'm a little worried that my philosophy is a little outdated cuz it looks like they've changed things in the last year or so. When I compare my teaching to other first year teachers, our course outlines look so different! [Laughs] We've compared, like, what are you discussing in class? And uh, well what's your outline look like? What're your tests like? We've talked about 
those kinds of things. We'll make comparisons, like holy shit you're so different than mine! Um... But it's fine.

See, my first year of ASL was very different from the current philosophy. When I was in first year we had one video test. The last test of first year. All the other tests were written. When I started teaching it was very opposite to the philosophy they're teaching. So I sat in on a couple other classes, Oooookay, got a feel for it.

Yeah, when I started out [teaching] I used to do that a lot more than I do now. Um. Sometimes even now when we get together to discuss things I'll be like, I have some students who'll just be like, I have students who are on their phones all the time. And one teacher is like, beginning of class, take their phones away. I don't really like the idea of doing that, but um I did like other teachers' suggestion to say, you're losing participation marks if you do this! So I have a lot of teacher suggestions in the back of my mind for a few different things that come up.

Nathalie worried about aligning with her colleagues and the broader program philosophy, especially since, as she later reminded me, she was teaching evening courses when few of her colleagues were around. Thus, she did not have access to her usual sources of insight.

Like Nathalie, David had been a student in the program he was teaching in and had briefly taught some two years prior. He was also concerned about having been out of the game for two years:

I taught two semesters. I taught a fall and a winter semester, and then I was off for two years. And in that two years I was socializing, maybe not excessively, but I was socializing. I wasn't teaching ASL. I was teaching 
ESL [English as a Second Language] for the deaf, but that's a very different thing. So I came back and I, you know, faculty has changed, who's running the program has changed and, ideologies shift. And I'm... confident in myself as a teacher, conceptually, I think I'm a good teacher.

He added that although he knew the program well from being a student, he did not have a firm grasp on how to adapt to being the teacher:

I haven't- I've been a [classroom assistant], I've learned myself, but I've never thought about what do I need to do for them as a teacher? So [the program supervisor] was like, here, do this. And I watched- I went and actually looked in, I watched other people's classes, and I looked at it and I had a list and I'm like, OK.

David made a point to check in with his colleagues to help guide his teaching. He had the advantage of previously being a teacher in his program and knowing how courses were usually run, although he recognized there had been a shift in his absence, and whom to contact when he needed help.

\section{Erica: "My little skill set"}

Erica had a different experience in her early days of teaching, as, like Michelle, she turned early on to formal teacher training for support with her teaching. She shared her memories of the training program, which gave a boost to her confidence and her skillset as she prepared to take on her first independent teaching position at a college.

It so happened that [the college] was looking to hire an ASL teacher and so I applied and was hired again without an interview or a formal 
assessment of my ASL and teaching skills. The idea of teaching at the college level with my little skill set was daunting. But I asserted myself and plugged forward.

Fortunately for her, her hearing supervisor recognized her potential as a teacher and arranged for her to take courses to improve her knowledge and skills. The training program comprised ten courses offered through a partnership between the Alberta Cultural Society of the Deaf (ACSD) and an interpretation college in British Columbia.

Although the program folded early due to a lack of funds, Erica managed to attend six or seven of the courses. She described the courses being run by a team of four experienced deaf ASL teachers, "same as I am", who "provided us with many examples of lesson plans and was very willing to share [] resources with us". She said that "[they] made up an amazing team and I can't say enough wonderful things about them" and that “these courses wouldn't have been as effective if they had been taught by hearing individuals." Her statements reflected her firm stance that ASL teachers must be deaf. She also created a DEAF CAN narrative - deaf people can be experts in their field, they can professionalize, they can work collaboratively to uplift other deaf people. She showed respect for her training leaders through her gratitude, alignment, and support of their work.

In looking back on the training experience, Erica credited that early course with her current success as a teacher:

It was eye-opening and helped me gain a deeper understanding, which led to feeling more confident in my teaching abilities, especially at the college level. Without that course, I wouldn't have been able to teach. 
In addition to boosting her confidence, the training gave her practical skills. She described one of the courses, which was about teaching to different learning styles:

In one particular class, we learned lesson planning because there were four identified learning styles within a classroom setting: those who are adept visually and receptively, those who need paper to reference because they struggle receptively, those who worked well hands on when they struggled receptively and didn't necessarily benefit from referencing paper and utilizing practise opportunities and games and those... I can't quite remember... Oh, I remember! Those who required preparatory materials in advance of the next class. We had to learn and understand how to meet the needs of the different learning styles.

I didn't realize and I was teaching out of the box, assigning homework, when in fact, I needed to type up all the materials, disseminate them, play games, provide some practise opportunities, summarize, review, have a discussion about what was learned in the class, go over the homework and then end class. It is imperative to work within the students' needs. Because I didn't know where the students fell within the four categories of learning styles, I had to make the class and materials accessible to all. I didn't necessarily know ASL rules and grammar or what a compound sign was and the difference between a noun and a verb or when something is legitimized and should be fingerspelled.

Same as- from what I have seen, there are many ASL instructors who haven't had any formalized training. They would typically teach vocabulary straight from a text but not necessarily expand on concepts. One example is that students often struggle with fingerspelling and the notion was to encourage the student to practise more. There are actually 
eight rules to fingerspelling which should be shared with the student to help them better understand and learn quickly.

The above narrative was telling in many ways. For instance, through the amount of detail Erica shared about the learning theory, she positioned herself as a knowledgeable teacher-expert and, intentionally or not, me as the non-expert. At the same time, she portrayed her early teaching self as unaware of the technical aspects of sign linguistics like the intricacies of fingerspelling, as if to showcase how far she has come since those early days. By recalling her course learning in such detail, she also belied how key that theory has been to her ongoing practice, a decade or more after she completed the courses.

In closing, the stories above collectively responded to my first research question, What are ASL teachers' pathways to the profession? In the final section, below, I pull out some of the patterns or threads that emerged from the stories shared in the above section in relation to the broader context of ASL teaching and the theoretical and empirical literature that informed this study.

\section{Threads From Chapter Six}

The stories in this chapter demonstrated the diversity of teachers' pathways to the profession. They had a wide range of backgrounds with ASL, where some were native signers but most were second language users who came to ASL and the deaf community by chance. For these learners, ASL had a significant and lasting impact on their personal — and later, professional—lives, particularly for those for whom ASL opened a new social world of possibility and barrier-free communication. 
For the most part, the teachers in this study started teaching ASL without or with little prior formal training or interest in teaching. Most of them starting teaching through friends and acquaintances in the deaf community. This pattern mirrored the literature in Chapters Two and Three that observed that teachers are often brought into their jobs without formal pre-service training. This trend reflected the narrative that ASL teachers should be deaf, where the above described hiring practice means that teachers can control entry into the field. It is unclear how the colleagues responsible for David's hiring spoke to this narrative, whether it was a resistance or a rejection of it in favour of an alternative narrative. Further research would be needed to address this question.

Many of this study's teachers' pathways to teaching were also defined by close mentorships and apprenticeship "under the wing" of a more experienced teacher. Peer-topeer mentorship among deaf people has been reported as a common workplace practice (e.g., Pine, 2012), particularly where other forms of support are lacking or barriers to success were high (e.g., audism from colleagues or supervisors). These mentorships have long been noted in academic publications to be a substitute for a defined professional learning trajectory; rather, they are the learning trajectory. At their core, they can be a way to carry forward, extend, and perhaps rethink common teaching practices and narratives of "good" and "bad" teaching, which are explored in Chapter Seven.

Learning about this study's teachers' early pathways in storied form was a humanizing counterpoint to the common narrative in academic literature about ASL teachers, which focuses on gaps and deficiencies from the time they enter the workforce. Academics have long expressed concern about the lack of standardization across ASL teachers' knowledge, qualifications, and practices. My own study, Doré (2015; see 
Chapter Three) found an enormous range of qualifications and training backgrounds, from novice teachers with relevant Master's degrees to highly experienced teachers with a single workshop of training and much in between. A recent report by the British Columbia ASL Articulation Committee likewise found that ASL teachers in the province were diversely trained and used a variety of teaching methods (Harding \& MacFayden, 2014).

At the same time, and one of my motivators for undertaking this study in the first place, regardless of their entry point, all of the teachers in this study came to dedicate themselves to developing expertise in their work through a variety of channels that are described in Chapter Seven. The value on-the-job learning, especially through collaborative learning channels such as mentorship (and more, as described in the next chapter), should not be underestimated, as it provided teachers with support and encouragement (key ingredients for success!) as well as an entry point into the discourses and practices of the professional community. The entry points started narrow, but expanded into a network over time as teachers develop familiarity and confidence in their work. In other words, these professional collaborations can help novice teachers to develop teaching and perhaps reflective practices that support their ongoing knowing-inaction. Ken's stories of his earliest teaching days are an excellent demonstration, where in his narratives, he made explicit some of the ways that his teaching had evolved over time through conversations with his mentor, his own in-class experiences, and, later, formal professional development. All of these experiences were, bit by bit, incorporated into his growing repertoire of experiential knowledge about ASL teaching and learning. 
On top of helping to develop a professional sense of self, a mentorship model can be an important contributor to a DEAF CAN narrative, where deaf people are invited to be masters of their language, authorities in the classroom, and contest the audist AGB narrative of DEAF CAN'T. Ken's story about his first days teaching alongside his hearing mentor who "made sure that the deaf always had the power in the classroom" is especially demonstrative. Likewise, Erica's surprise at how much she did not know or intuit about ASL teaching until she attended a training showed the extent that teaching can empower over time and help teachers to lay claim to knowledge about their own language and professional practice.

The above observation is in line with recent scholarship about the role of ASL teaching in deaf lives. Sign language instruction was and continues to be a significant career path for deaf people (McKee, Rosen, \& McKee, 2014), but it has also been described as a form of resistance to hearing hegemony. Robinson and Henner (2018) reproached higher education institutions with ASL programs for embracing the popularity of ASL courses for financial gain while simultaneously neglecting their ethical responsibilities to students and faculty with disabilities and deaf and signing communities. The authors disputed the practice of hiring of non-deaf teachers to teach ASL because "it is a resistance language of a marginalized community" (par. 11). I explore this debate further in Chapter Ten.

Related to this, the novice teachers in this study (David, Nathalie, Nick) who were hearing or raised oral relied less on support from the deaf community in their early days, and more on their workplace colleagues. Meanwhile, the experienced teachers who were mostly raised signing (with the exception of Michelle) considered their deaf community 
peers invaluable resources in their developing teaching practice. A parallel scenario was observed in McDermid (2009), where the division between deaf and hearing and faculty was pronounced in their different relationships to outside communities, where deaf faculty were significantly more involved with local deaf communities and hearing faculty with professional communities (e.g., professional associations). It is difficult to know whether the habits (so far) of the novice teachers in this study were a function of their oral-based language background, their limited time in the profession, or their pre-existing relationships with colleagues in their respective programs, or whether their patterns will change over time as they become more embedded in ASL teaching. Either way, since the three of them had previously been ASL students themselves, they were already undoubtedly informed in their teaching by their prior learning, thus drawing on prior experience and observations. Otherwise put, their personal theories about ASL teaching and learning had been growing long before they were teachers.

This question of teachers' early support systems is especially interesting in the context of ASL teacher training in Canada. As described in Chapter Three, in Canada, formal ASL teacher training opportunities such as workshops and certificate programs are most often organized through local, provincial, and national deaf cultural associations, e.g., the SLIC. An exception is the long-running Signing Naturally ASL instructor training program run by the CHS. The CHS is not a deaf cultural association but nonetheless has as its mandate "a barrier-free society" for deaf and hard-of-hearing people (CHS, 2020). Their Signing Naturally instructor certificate seemed to align with many deaf cultural organizations' trainings. 
Thus, in Canada, it is likely that any ASL teacher who seeks formal training will do so through a deaf cultural mandate and, as such, become socialized into a professional community that is tightly bound up with the deaf community. In the USA, the primary sign language teachers' association, the ASLTA, exists separately from their deaf cultural organizations, which may make it significantly easier for an ASL teacher to remain uninvolved with the deaf community. Further discussion of professional development has been reserved for Chapter Seven, which I turn to next. 


\section{Chapter Seven: Pathways Through Teaching}

The previous chapter took a closer look at teachers' stories about their pathways to ASL teaching - how they came to ASL and the deaf community, and what people, events, circumstances, and even emotions surrounded their entry into their work. This chapter moves forward along the timeline and addresses teachers' pathways through the profession. For the newer teachers, the chronological distinction between "early" and “ongoing” career is difficult to trace and I made no attempt to draw a hard line. Here, I explores teachers' experiences throughout their careers - in and out of the classroom, in learning and development, in relationships with colleagues and the community.

I begin with teachers' stories of "good" teaching, the sacred stories (Clandinin \& Connelly, 1996) teachers tell about ASL teaching generally and about their own teaching specifically - how they aspire to be or not to be as teachers. Their stories show the many hats a teacher may wear, from guide and sage to coach and motivator. Following that are be teachers' stories and reflections on how they did not always live up to those expectations, how they were "imperfect" teachers. These stories also reflect teachers' conceptualizations of how they ought to or wanted to be. To understand a bit more how their aspirations and faults play out in real terms, I then present stories of the classroom. Several teachers referred to Signing Naturally, but in the Michelle's case, for example, magic and play featured heavily. Next are stories of participation in formal ASL teacher training programs. I focus on four teachers who each attended a different training program at some point in their career. I take the opportunity to look briefly at the programs to understand how they were oriented (what informed the practices they promoted, for example) and, by extension, how the profession has worked to build up its 
members' knowledge and skills base. To close the storied part of the chapter, I present stories of the interactions and collaborations that have shaped teachers' practice, positively and negatively. These stories show an assortment of successes as teachers came together to achieve cohesion and challenges as collegial partnerships fell apart or failed to come together in the first place. Erica and Donna's stories at the end of the chapter about the difficulties of replenishing the workforce with new "young and eager" teachers are a reminder of the precarity of the profession.

Overall, although common in academic literature, audism and discriminatory behaviours in the workplace were not strong threads in the stories of the teachers in this study, with the exception of Ken's distressing experiences with interpreters and the occasional hearing ASL teacher (whom he described as "shameless"). Instead, the most significant challenges they faced were with other ASL teachers and peers in their deaf communities. This may suggest that unlike for deaf employees who operate in a hearingdominated environment, audism was not a significant workplace obstacle for the teachers in this study because ASL teaching was largely governed by deaf people. That said, the stories shared below show how varied deaf communities can be. If my seven teachers were in a room together, I suspect there would be ample lively discussion and debate about best practices, the ideal teacher, training methods, and so on.

A scan of the table of contents shows that Erica and Ken are dominant voices throughout this chapter as their stories appear in nearly every section. While I was tempted to reduce the number of their stories that I featured, Erica had a wide range of insightful experiences worth sharing; Ken's stories, meanwhile, were so richly told, powerful and unique, that I opted to keep more space open to accommodate them. I have 
attempted to interweave their stories in a way that was intended to support and not overwhelm or distract from the stories of the other teachers. In several instances, because Ken's stories in particular were often lengthy, I discuss them in reduced form.

The first stories of this chapter, below, encompass stories about values and morals related to ASL teaching (Elbaz-Luwisch, 2005), role models, teaching philosophies, and formative experiences that informed teachers' pathways through their teaching.

\section{The Good Teacher}

Nick's reflections introduce this section because one of his seemingly unrelated recollections of a recent class event indirectly displayed his values and aspirations about “good" teaching. Erica's stories, meanwhile, explicitly state her stance on deaf-only hiring and teachers maintaining high standards for their own professional development. One of Donna's priorities for herself was to properly prepare her students for their lives outside of the classroom and in the deaf community. Ken echo many of Erica's views and shared stories that displayed, through affirmation and opposition, his teaching philosophy.

\section{Nick: "We're taking another step up the stairs"}

As described in the previous chapter, Nick was new to ASL teaching in the university. Still, his narrative below suggested that he had a vision of how ASL learning should look. I present the narrative in full form, as it would have been less effective otherwise.

I'm working on improving the syllabus... The- what I teach them. Cuz it's my first time doing it with university kids. And having been in their shoes 
before and now being in these shoes... I kinda know where they are, where they're not at.

Like, I do have some kids that are... like this one kid today, they were prepping for their presentations next class and he just sat there and didn't sign anything while the rest of his group was signing. I gave them the leeway to talk to each other today because, you know, to help each other figure what they're gonna say, but he just kept his mouth quiet and his hands were, like, curled up in his arms, not doing any signing at all. So I know that he's struggling or...

Like, sometimes, students, you can tell it's like a cultural thing for some of them where in their culture they're not as expressive uh... and then ours we're very expressive, in deaf culture. You have to be expressive. So you could tell he's not as expressive as he should be or could be. It's up to me to find a way to pull them outta their -break, as I say it, break them outta their shell. I told them that at the very beginning of class, You have to be expressive, get out of your shell. You've gotta do that if you want to excel in this language. For now, it's a first step. First step is just first semester. Then, second time around I'll add another step forward. We're taking another step up the stairs...

And then- like, the kid sitting there today, and then there's the ones that comes up to me like, How do you say this? And I appreciate those students more because they're being more... curious and want to build on the knowledge and that. And I respect that. But then when you sit there with your arms crossed or don't even show up for class then I don't... It's not that I don't have respect. I just don't... appreciate your effort as much as I appreciate their effort. 
The first point to note is Nick's decision to give students "the leeway to talk to each other today". By emphasizing that the allowance was "today" and immediately offering an explanation of why he felt it was important to let the students talk to each other, Nick recognized the prevalence and importance of the no-voice rule, but chose to exercise his own teacher judgement and agency by violating it. His permitting speech in the classroom also showed his concern for building up students' confidence and comfort with ASL by setting up an environment where students like the one with the crossed arms could "get out of their shell" and take steps forward in their learning, one at a time, "up the stairs". He portrayed himself as a coach and a guide in learners' process.

As he said above, he also appreciated students who made an effort. He recognized that their investment in the language and their own learning was the only way to get results, especially since he was spread thin:

I don't- I don't like seeing people not do well in signing. Especially when they're trying to learn it. But at the same time, I can't grab your hand and walk you through it all cuz I have over 50 kids. I can't push everybody. You know... I can't give everybody a 1-on-1. I can only push so much, so far. Like, you've gotta be, as a student at school, you've gotta be able to do it on your own. I've been in your shoes. I've gotten overwhelmed before and, you know, battled my way through it. So you can do the exact same thing. I shouldn't have to push you, or grab your hand and walk you through it. You should be able to... you're in university, a whole other level now.

Here, Nick pointed to a reality of a successful ASL program: classes can get big and difficult to manage. For instance, Ken related how when the courses he was teaching 
started to exceed 20-25 students he needed to bring in another teacher and split the class in two. Nick's response to his group was part empathy — he remembers being a student and being overwhelmed - and part firm that university students should be held to a high standard. In doing so he was pushing back against discourses of teaching as a maternal role and instead holding to the stance that a good teacher is one who can successfully direct—not provide—-motivation and effort.

\section{Erica: "Time is always changing"}

Near the end of our time together, I asked Erica if she had any last thoughts that she had not yet shared. She thought about it briefly and then said:

I really cherish ASL! I believe any deaf individual can teach ASL. Hearing people should NOT teach ASL. ASL is the language of the deaf and should be taught by the deaf. That is my belief.

She quickly added that those deaf teachers must also hold themselves to a high standard as professionals:

I strongly encourage deaf ASL instructors to keep up with their education and ongoing learning through courses and workshops because we know time is always changing and therefore they need to update their skills. My boss who happens to be hearing and a social worker shared with me that the members of the [ ] college of social workers are required to take one or two courses per year to keep current with their knowledge and skills, which made me think that should apply to ASL instructors too. We too need to keep up to date with new readings and knowledge to keep current because as time changes, ASL changes. 
She went on to explain to me that "ASL was really only recognized as a language in the 1970's by Dr William Stokoe who was a researcher and finally published in the 1980's, not too long ago". She described the strong need for continued research to keep up to date with "all the changes to ASL over the years and add the new information and rules".

Erica's vision of a "good" ASL teacher is one who is deaf as well as prepared to make a commitment to the profession by seeking to improve their skills regularly and staying current on the literature and resources in the field. Her stance speaks to earlier commentary about deaf-only hiring and teaching as a DEAF CAN opportunity, which is also prominent in Ken's upcoming narrative. Like Donna and Ken, Erica was active with her provincial deaf cultural society and so was likely more aware than most of the opportunities for professional development for ASL teachers. But as Ken experienced, not all cultural associations are effective at spreading the word about opportunities, which may make it difficult for teachers outside the "inner circle" to fulfil the mandate. Likewise, as Erica herself observed, there is limited research to support even ambitious teachers.

\section{Donna: "It's my responsibility to let them know"}

Donna had been teaching for well over 20 years by the time of our interview. She had seen massive change in the profession. She described the big changes from before the ASLICE certificate was established to after, where all of a sudden, the certificate gave teachers more direction, motivation, support, credibility. She also talked about needing to keep up herself with language change, especially as people move around and encounter regional variations, so she was well-prepared to prepare her students. 
For Donna, she was not the only teacher for her students. She also saw a role for the deaf community in building ASL learners' skills, although she recognized that some ASL programs have their reasons for avoiding sending students into the deaf community. She considered first-hand experience to be a rich source of insight for learners into the deaf world:

I think it's important that they come out to things and they understand the deaf world a bit better. I think hearing students should be volunteering, too. It's good for their signing, it's good for them to get to know people and so I believe it helps. Looking back at myself, when I started- when I first moved here and I didn't know anyone I volunteered. I met so many people, I got involved with the local groups. I think for hearing students to be forced to go socialize can be asking quite a bit, but I think sometimes volunteering is an easier way in.

She explained that her stance was probably because she grew up in Eastern Canada before moving out West. When she moved she needed to adapt to the new regional signs.

Sometimes when I'm teaching, I forget what the [Western Canada] sign is, I get stuck on the [Eastern Canada] sign. Like I grew up signing- what looks like SOON, for me is SLIPPERY [SOON flicked off chin]. It happened recently that I was at a workshop and someone used the sign SLIPPERY [SMOOTH] and I was like, oh yeah, thanks I had forgotten that. So it's important to learn what other signing communities use. You can't just stick to the one you prefer. You need to adapt to the community.

And sometimes there's more variation than teachers realize. Like USE at the hand or in the air. One group does it one way and the rest something 
else. Teachers need to tell their students that they'll see both, sometimes more. They need to know so they can communicate out in the community. I always let my students know there will be lots of variation in the signing communities they go into. I try to give them exposure to as many variations.

Like, recently, yesterday I was teaching them, looks like iPad [other direction] but in California it means rude. We do RUDE at the cheek. That's what I use. So I let them know that that's what they'll see in the book, but here people use something else. It's my responsibility to let them know otherwise it can be really confusing.

She saw ASL teachers as being responsible for keeping up to date with new signs and ensuring her students were aware that there can be significant variation between some signs.

\section{Ken: "My philosophy is about giving"}

I reserved Ken's stories for the end of this section because his reflections on his philosophy of teaching and how ASL teachers should be were extensive and often intertwined with views on how teachers should not be, which were equally telling.

As a first point, Ken values a teacher who is energetic with the ability and aim to inspire learners. In his early ASL contract, he found his energy for teaching was not matched by his colleagues. Ken's storytelling was vivid, and so I present his narrative in full form.

I can't stand it there [the interpreter school in B.C.]. I didn't want to work there. It's no good. Their philosophy of teaching, their major... a bunch of things... It's... it's... How do I explain it? It's hard to say exactly... 
Like... OK, let me see. It's like they're really laid back... informal-like in their teaching. There's not a lot of intensity to the program. They don't push their students. It's not a systematic program. There, teaching is just giving some handouts and then sitting back. Maybe a little grading.

Really, the teachers were always sitting back, passively... and that's not my style. I prefer to roll up my sleeves and really get in there. I would record students and review their videos with them. Like, see your facial expressions here? And I'd press pause on a frame to show them what I meant and let them see, like, ah yes, you're right. And then I'd explain in more depth. I'm a very hands-on teacher. Very hands-on. And I always have been.

Over there, those people at [the college], that group of teachers, they're much more money- and time-focused. That's what they care about. And it shows! I think the most important thing is that if somebody is going to become an interpreter later on, your priority as a teacher should be to elevate their skills to a high level so they can become quality interpreters. That's what I believe. That's my approach. [The college teachers], their approach is more... more hands-off, more detached... and I- I just can't. It's totally opposite to my approach.

The director at [the college] was an old man. A hearing man. He could sign some. He came in and was trying to rework the program, to change things around. Eventually he left the position and [a new director] replaced him. A few times I went into his classes to observe him teaching and he was very casual, kind of lethargic. It's is very different from my style and so I felt like we weren't really on the same page. My style is more... pushing students, encouraging, getting them to get real-world experience. And I definitely don't teach the same things, again and again... I'm always changing my activities. Part of the reason I do that is to avoid cheating - 
students copy answers from past years and so teachers who use the same thing every single time, well, I think it's asking for trouble. I changed my exams every year, but the others would complain that it takes too much time to do that, why not reuse what I have, blablabla... but I think it's totally worth it. And it's your responsibility as a teacher, too.

He described how the program had not undergone a curriculum review in many years or updated their materials "since the year 2000" and was long due for an overhaul. He was especially concerned with USA ASL vocabulary not being converted to Canadian ASL, as this brought interpreters' language further from the local community's. He felt the teachers in his former program were not upholding their ethical and professional duty. They were also, according to him, inefficient in the classroom.

There was a deaf teacher [in B.C.], I remember, who was so motivating, so passionate. You could see the student absorbing his energy, his positivity, his enthusiasm. Meanwhile, the Gallaudet teachers you see here [ ], they're like zombie teachers. They're slogging through their courses, passing around the odd handout. Writing a few things on the board. No life. It's frustrating. A student has a question, they answer, like, Mm-hmm, yup, good, fine, okay. There's no spark, no debate. Or... inspiring the students, discussion, enthusiasm...

They don't explain things to the students. They don't have a philosophy of teaching that draws students out and pushes them forward, that they can leave class with... Sure, sometimes students can be challenging, and some teachers don't like being challenged, they're afraid of it, they don't know what to do about it. But come on, you're a teacher! This is learning! You need to keep learning. You need to pull yourself out of your shell and 
develop yourself, develop your ideas, challenge yourself. That's how I feel about teaching. It's very close to my heart.

As an initial note, Ken uses "the Gallaudet teachers" to refer to those teachers who either attended Gallaudet University themselves or follow the materials and methods of teachers who did. Ken associates the Gallaudet teaching mentality with dominance—-dominance of Gallaudet teaching methods over all others, the USA dialect of ASL over local ASL/MSL varieties, dominance of English in ASL teaching, and so on. I unpack Ken's stance on "the Gallaudet people" later in this chapter.

Ken's disdain for handouts and other static teaching resources was recurrent throughout our interviews. He described one "Gallaudet" teacher in the classroom:

It was one sheet of paper with a bunch of writing, explanations about facial grammar or something, and some little pictures. I couldn't figure out any of it! I thought it was ridiculous. This is how they teach? All writing and no signs?

He repeatedly emphasized the importance of maintaining alignment between teaching methods and the subject. As in, sign language is manual-visual, and so should be the teaching methods. He resisted English in the classroom also because, as described in Chapter Six, he noticed students had difficulty differentiating ASL and English syntax. Another implication in Ken's above narrative reflected Erica's earlier view: ASL teachers should take their work seriously and avoid stagnation or passivity in their work. Ken was highly motivated to shape strong signers in his classes because he was strongly driven to improve the quality of interpretation services (as described further in Chapter 
Eight). His resentment for his former colleagues was a form of blame for the current quality of interpretation.

Ken also resented his colleagues' resistance to investing more time and effort in their teaching. He said that maintaining a strong course design and reflexive practice were important aspects of teaching work and "your responsibility as a teacher, too". He gave several examples of how he put words and thoughts to action in the classroom and how those reflect his philosophy of teaching centred on "giving":

I teach 30 hours over 3 months. Their assessments are video-based. They submit a video of them signing, and I watch them and take some notes. Then we have a one-on-one meeting where I give them feedback so they know what to improve. Later in the term we do something fun; we look at the video they submitted at the beginning of the course and compare it to their last video after three months. It helps to show their improvement over time. They can keep their videos, too. People really like that. Some of them find it hilarious to look back at their early signing, all choppy and slow... Anyway, I think it's motivating for the students to see how much they've improved from the beginning to the end.

My philosophy is about giving. Giving students something to work with. But also, it's teaching and learning together. It's not I know everything and the students know nothing. I really try to approach teaching as an exchange of ideas. You know, I don't get too protective about what I know, I don't take myself too seriously, I don't assume I know better than everybody... That's the fun thing about workshops. You get all of these ideas from people, you can wake people up who are falling asleep because they've lost their energy for teaching. Like, when I go to workshops, I make sure to take notes, then I go home and reflect on it, and then next 
class I try to apply it. And I know lots of other teachers go to the workshops and then they don't want to discuss it again or look at their notes. But you need to make the learning your own. You know?

When I coordinate workshops I'm not standing still up at the front, like, the all-knowing- I'm going back and forth, trying to engage with the people in the audience. I don't shut people down. But I know there teachers I run into that are like, Don't tell me what to do, don't tell me I'm right or wrong. They shut themselves down. It's a lousy attitude.

Thus, for Ken, a "good" ASL teacher is one who has professional integrity, takes students' needs seriously, and is aware of the import of their work in the bigger picture, such as the quality of interpretation.

While the ASL teacher-participants expressed a variety of features that made a "good" teacher, many of them also reflected on "bad" teaching, or at least not fully meeting the standards of "good" teaching, whether themselves or their peers. I describe those instances below.

\section{The Imperfect Teacher}

Being an imperfect teacher was part and parcel of many teachers' stories from the previous chapter about their early experiences with teaching. Ken, for example, in Chapter Six, described his immense development as the years went on, in large part to the initial mentorship of his hearing partner and then later other teachers, leaders, and scholars. When he reflected on the journey, he attributed his early shakiness to his past educational experiences and on cultural differences between himself and his hearing students: 
Things like teaching theory, classroom management, all that, I didn't know about any of it. Growing up, in my schooling, the teacher was really laissez-faire about teaching and informal, kind of disorganized. So as a teacher I started modeling myself after them. That's what I knew. And my partner saw that and said, no, no, you need to be professional if you're going to teach. So, I had to change my attitude and behaviours a little bit. And until then I hadn't realized what I was doing, so I'm glad he said something.

I started noticing things like, I'd go into the classroom and joke around with the students, and some of them didn't take it too well. Sometimes they'd get offended or upset. This one time a woman came in with this big dress and I said, man that's a weird dress! It looks like a clown outfit! And of course, she was upset by that. My partner jumped and told me, whoa, you can't be making fun of your students like that. But I didn't know. Nobody told me. That's what I experienced. That's when I started going to workshops and conferences and talking to other teachers and learning about different ways of behaving in the classroom. Bit by bit, it got more obvious that I had a lot to learn about teaching. So I got to it, started learning about how to improve myself as a teacher.

Ken was the only teacher to point to his childhood educational experiences as being impactful on his initial conceptions and behaviours in teaching. As he said, he modeled them, "That's what I experienced". He implied that without his partner's intervention he would likely have carried on teaching as he had been and perhaps not have been inspired to pursue additional professional development.

Several other teachers shared reflections on their pursuit for improvement, including David who described his attempts at hitting the right note in his teaching, and 
Nathalie who in our interviews was still processing the ways in which she was working on herself as an "imperfect teacher".

\section{David: "Swinging towards the joy"}

In our conversations, David did not often speak in personal anecdotes about concrete events; instead, he tended to abstract his experience and speak about them at a conceptual level. When he reflected on his successes and failures as a new teacher he again related a high-level account of how he was in the process of finding balance in the classroom:

And when I came into it the first time, I was very strict about, This is what we're doing, and it'd be like 30 signs every day. And thinking about it now it just terrifies me because if I had taught me I would not have gone on to second year. So I'm- I'm impressed by any student who had me as a first year teacher that time around and went on... because I was gruelling! And my expectations were so high! In part because I took intensive first year. In the summer. So it wasn't even four months, it was three months, because it's a short- It was intensive. But I thrived. Right? I really thrived in it. But not everyone did. And I ended up making my first year nonintensive a bit too intensive.

So. This time around I spoke- I came back and said, I've been out of the loop for a while, what are we doing now? And they said, we want you to focus on this thing. I was like, OK. And I went too far the other way. I think... Other people disagree with me, but I feel I went too looseygoosey. Maybe I'm wrong. Maybe I'm hyper-critical. I knew I didn't want to repeat [last time]. 
What I didn't do well was making sure I had a schedule in mind. Next time I'm going to try to hit the middle, leaning more towards loose and play. Because- ... There was a lot more laughter in the classes I taught this semester than there was in there. And that was important for me. Because, coming back to it, it's like, having the experience teaching deaf immigrants, the smile of communication, or the laugh of communication, was when there was a real connection happening. When there was actually joy in the moment. First year it was kind of, Uh why am I here? Why am I doing this to myself? It's not- I mean, maybe you're learning but it's not fun. It needs to- you can't keep up the energy to keep doing it unless there's some joy there. So that has to be a part of it. So I'm- I need to hit a more structured approach, but it still needs to be swinging towards the joy.

This story showed David's ability to critically self-evaluate as part of a productive reflexive practice. Several of his values about teaching and learning were also on display, including the importance of connecting with students and fostering an enjoyable learning environment.

\section{Nathalie: "I do what I can"}

Nathalie was also open about her limitations and her occasional struggle to meet her own expectations, particularly about her abilities as a signer. She explained that she had been signing for ten years, she was not a native signer, and she had only recently returned to socializing with the deaf community. Her response was to seek input and support from those around her, from colleagues to deaf friends and acquaintances from around the community, to the Internet (with caution):

Every once in a while I have to tell [my students], 'I don't know' to their questions. 'I don't know' is fairly common, I'm sorry! I don't know 
everything. But, they deserve honesty. Sometimes I'm like, I don't know. I'll reach around, I'll try to find out. If I don't, I'll be like, well, there's something online. I can't verify its accuracy so take it with a grain of salt, but that's what I was able to find. ... I do what I can.

I will sometimes correct my signs based on OK, other people have been telling me that we either don't sign this like that anymore, or nah nah nah don't use that one, this one is better. And... ... I'm just honest with my students. So if I taught them something wrong, I'll be like, OK, I made a mistake. [ ] Use this, don't use this. Sometimes it's like, OK these signs are acceptable. Going future, if you're gonna do second year and third year, you're gonna wanna sign them this way. Well, I'm only teaching first year. If other teachers have a different way of signing something and the students are going to have to sign that for their future years I want them to be aware of it. It's not that I always think my way is the wrong way, but I'm going to accept criticism if I get it.

Although she had previously expressed feeling self-conscious about her signing, when explaining the above, Nathalie showed only a willing commitment to self-improvement. At one point she explained how she used to be more concerned about her signing skills, but has since come to terms with her "imperfections":

So... I was worried I wouldn't teach things exactly as they should be. Like, I learned a sign wrong or something from someone, or I taught something wrong. And I'm like, you know what, everyone signs something incorrectly from time to time. Every single per- deaf person, every single hear- every single person I've ever met has signed incorrectly, and it's 
hilarious. So like really? Yeah, it's going to happen. You don't need to get so worked up about it.

Everyone who's sat on my class and observed my teaching has told me I'm a good teacher, so maybe I should take their word for it. [Laughs] I think I like seeing myself as an imperfect teacher... might not be the worst thing ever either. It makes me wanna be better, makes me more able to admit when uh something maybe wasn't completely right. It makes me.... maybe easier to approach for students. I dunno... I don't know. Yup. [Laughs]

Her repeated laughter, which I read as somewhat nervous or apologetic, suggested that although she said she was coming to terms with some of what she perceived to be her flaws as a teacher, some hesitation remained. Throughout her stories, she showed a growing sense of herself as a teacher, a growing confidence as she developed a deeper understanding of her work and some of the contentious aspects of it

In the above section and the preceding one, I presented teachers' stories about "good" and "bad" (or imperfect, in-progress) teaching. The stories that follow explore some of the actual practices that teachers engage in their classrooms.

\section{In the Classroom}

As I mentioned in Chapter Two, the literature has identified ASL classroom practices as being diverse and informed by outdated theories of language teaching and learning. Yet studies have also found that teachers overwhelmingly use the Signing Naturally textbook series, which to some extent provides at least a baseline level of standardization. As a reminder, Signing Naturally is a multi-part textbook series that was designed to double as a teacher training guide for people interested in teaching ASL. 
Although not addressed empirically, anecdotally, deaf teachers want to support deaf authors (the authors of Signing Naturally are deaf) by using deaf-authored materials (Thoryk, 2010). One of the teachers in the present study, Donna, said this outright when I asked whether she used other textbooks such as the hearing-authored Master ASL!:

I haven't used Master ASL! I know it, but it's a hearing author, whereas Signing Naturally they're deaf authors, so I would rather support that, right? I stand behind deaf publications. I mean, that's my personal philosophy.

The use of textbooks and other materials in ASL teaching could be political, philosophical, personal. I did not push for further justification.

Although generally speaking there is limited in-class observational research to corroborate that teachers' classroom practices are diverse, the prevalence of Signing Naturally should suggest that there is at least some degree of standardization in ASL classrooms. In the present study, several of the teachers in this study used Signing Naturally but in myriad ways, thus proving both points true. Several teachers did not use a textbook at all, and so our discussions of materials was exceedingly brief (somewhat replaced by discussions of their classroom practices and teaching philosophies). While I in no way propose that the stories below are representative of other teachers and classrooms, I do hold that they provide snippets of insight into the diversity of in-class teaching experiences. 


\section{Erica: "I adapt it and add my own stuff"}

For Erica, Signing Naturally was a staple in her classrooms. She was happy that a new edition had been released: "It is much better. They have included far more vocabulary, information, as well as cultural information and it is great." The old edition, she said, was "slim" and "quite limited".

She described a typical class using the book:

When I'm teaching, I open the class with a handout from the textbook, from Signing Naturally. That's what I use. I open the class and get started with some vocab from the book. When we're done with that, we do a game- rather, it's more like a kind of partner work where they ask each other questions about a topic I give them. They practice, swap partners, and keep going. When they're done with that we usually watch from the $\mathrm{CD}$ that comes with the book. The CD has- sometimes it's too fast for the students, I noticed.

So we watch little pieces at a time. A clip, stop, discuss it, I answer questions, keep going, pause. We do a bunch of that. And to end the class we do a review of what we did today to make sure everyone is on the same page about what we learned to day and people are feeling good about it. And then at the very end I give them homework and info for how to prepare for the next class.

I definitely follow the book. But I adapt it and add my own stuff. For the students. I want to make it engaging, right? More interesting. I know the students sometimes find it hard to connect um, what they see and what they do with their own bodies, so I try to help them with that. Give them lots of opportunities. But I always use the book as a starting point. 
From her explanation, Erica's modifications to the book were fairly superficial. As she was telling me the above she showed me her copy of the book. It was tattered, with the covers ripped off (to make it easier to hold open, Erica explained), and completely covered in sticky notes and highlighter markings.

\section{Ken: "I use the book as a guide"}

Ken also used Signing Naturally as the basis of his teaching after trying out many other textbooks and resources. Several years after he started teaching, Ken completed a teacher training program (described in the following section) that converted him to the Signing Naturally curriculum. While Erica was mostly satisfied with the curriculum as-is, Ken was more critical:

For me, I've been teaching long time. I've used books like the Joy of Signing, I'm not a big fan... The ABC book [i.e., Baker-Shenk \& Cokely, 1990], not a big fan... And then there's Signing Naturally. I started teaching with it and loved it. Most of it is really great. It gives good explanations, good activities. Except it's also a huge struggle sometimes because the signing is all USA signing, and it causes a lot of confusion. There are a whole bunch of signs that need to be changed to Canadian signs for it to work. Anyways, there's also the Green Books. Those are really good, too. They talk about sign language structure... the course is presented in units... I think students get a lot out of those.

As he repeated several times during our interviews, Ken was careful to tailor his teaching to the language and needs of his local signing community: 
Over the years what I've customized Signing Naturally to suit my needs. I use the book as a guide, the basic organization. Most of it is great, but some of its everyday language it really odd, doesn't make sense. Like, do you really know how to buy and sell a car? Is that an everyday event? That happens maybe a couple times in your life and that's it. They have a whole unit on it. That doesn't seem useful to me. So, I supplement with my own ideas about what everyday language is important. Not things like selling a car. Compare that to other everyday activities, like cooking, or cleaning, or getting dressed.

Since Ken was firmly in favour of visual materials, he also modified the mode of delivery to not be exclusively static and English:

Some of the changes I've made I put on YouTube where I show the sentence and I sign it. They're all on YouTube, all public. Take a look if you want. What I try to do is, for example, show different signs for different things. Like, different behavioural things. Hearing parents with a deaf child might need to know how to discuss behaviour problems. If their child is getting picked on, they want to be able to discuss it and teach things like, 'Stop picking on me' or 'Stop bothering me'. So I give them a list of sentences and I teach them during a teaching session, but then I make these videos for them to refer to later because realistically, they're not going to remember everything. I post these videos on YouTube and that helps them review their list of sentences I gave them before. That seems to be really successful.

He then shared an anecdote about an unintended impact of his videos: 
Actually, there was this one time that my partner and I went to IKEA and a random man came up to me and said, My name is, and he spelled his name. And he was like, I've been learning sign language from you on YouTube. And I was floored. What? You've been watching my videos? The guy goes, Yeah! And he was signing pretty well, we were having a full conversation. And he said it was all from the videos I posted. It was really impressive. I was shocked. I had developed it just for my students, but man, if other people want to use it for their own learning, sure, why not? Cool. I'm sure there's a better way to do it, but still. It was neat. It was a cool experience.

All in all, Ken used Signing Naturally as a foundation for his classes. He adapted it as needed based on his knowledge of sign language linguistics, sociolinguistics, historical linguistics, and more that he gleaned from the many workshops he has attended over the years.

When he worked in the college interpretation programs, Ken said he was constrained in the curriculum because it was designed to maintain some level of standardization amongst graduates. At the time of our interviews, he ran his own teaching business and could better tailor his teaching to his students' needs and his own agenda. In his commitment to creating a classroom environment that fostered respect and professionalism, both skills he felt were essential to the future interpreters he was teaching, Ken had a series of class rules. These rules, he reasoned, lent rigour to the classroom. His philosophy that students should be held to a high standard came up several times throughout our interviews. It related to his goal for students who were on their way to becoming interpreters learn key professional practices, like dressing appropriately: 
My philosophy of teaching is very different from other teachers. For example, I come into the classroom, I've got my classroom rules and I make sure everyone understands them. Rules like wearing solid coloured shirts, no hats in class, no sunglasses... Let's see... Things like, gloves or jewelry or other things on your hands. These kinds of things are really important for beginner signers. It's hard to see their signs if they have, like, a striped shirt on. Anyways, so I have rules that like for my classes. Obviously, no talking... No phones, no texting. I need their eyes on me, to stay focused. Rules like that. It's happened a few times that I'll catch a student playing on their phone and I tell them, Put your phone away, put your phone away, put your phone away... Eventually, I just ask them to leave class.

He mentioned that other teachers did not do as he did and later described how interpretation students were being passed with poor etiquette, poor attitudes, and substandard skills. Some students, he said, not his, were being passed after promising sexual favours in exchange for a passing grade. Other students were in romantic relationshipssome even marrying - their instructors and likewise graduating from the program with dubious skills. Ken felt so strongly about his program's poor standards that he filed several complaints, which eventually led to him being (unlawfully) dismissed from one of his teaching jobs. His struggles with colleagues are revisited later in this chapter.

\section{Nathalie: "I have my methods"}

Nathalie's stories of her classroom methods were an interesting counterpoint to Erica and Ken's since she did not use a textbook or indeed provide her students with any written resources as per the philosophy of her program. She was instructed to avoid even 
dictionaries because her beginner-level students were unprepared to evaluate whether the signs they found were suitable to the local signing community. Nathalie pushed back on this point somewhat, arguing that tools like dictionaries can be valuable with a proper disclaimer:

For the ASL my students learn, I want them to be aware that signs I pick up from anywhere... might be valid signs in general. But... it could be a sign from another language, a sign from another dialect of ASL... I let them know these things, ohhhh yes. Some students are like, oh can we get a dictionary or something? And ... when I first started teaching, no. I was told oh it's a bad idea. Really? I think the students should have the option to look through things and be like, oh right, yeah that's what we learned in class, I recognize that. So... Well, if you give them dictionaries you'll give them the best ones that you know of. Whereas if they go looking themselves...

When I asked about her reaction to not only dictionaries but all written materials being discouraged in her program, she was mostly supportive:

Um, I like the idea of not writing. When they [the program] threw out writing tests, I like it cuz it takes English out of the classroom somewhat. They're- they're not using English all the time, or if they are they're fingerspelling, like, they're moving- using their hands, they don't have their pens in their hands all the time. So I like that.

At the same time, Nathalie uses English as a tool for giving feedback because she considers feedback an essential part of the learning experience: 
For midterm feedback I do sit them down 1-on-1 and give them feedback, in English. In my office. And the reason I do it in English, like other teachers have asked me oh do you do feedback in English or ASL? Really, if the student is comfortable and wants feedback in ASL, oh yeah. I will. No problem. The reason I do it in English is I want them to really understand... and the students who are struggling the most in ASL generally feel way more comfortable with English... And they're the ones who need the feedback and to understand the feedback the most.

Similar to Nick's earlier narrative about his allowing occasional spoken English into the classroom, Nathalie's explanation also defended her decision to use spoken English in her teaching by arguing that English is the best way to deliver feedback to students and that feedback is essential to learners. Like Nick, Nathalie saw spoken English as a mechanism to put students at ease and better meet their learning needs. Unlike Nick, however, Nathalie only used spoken English in her office, outside of the classroom. Her use of voicing was therefore not technically a violation of the "sacred" no-voice rule, which referred to voicing in the classroom. Nathalie referred to her classroom a safe space for her students, where she tried as much as possible to make sure her students felt comfortable. This tone continued in our follow-up discussions.

\section{Michelle: Costumes, games, and magic}

I spent most of my hours with Michelle looking over and discussing her lesson plans, slide decks, activities, and teaching props (of which there were many!). Her lesson plans and activities were based on several sources: YouTube, ASL websites, textbooks, activity books, resource books (dictionaries, etc.), and readings about ASL linguistics 
(some empirical, but mostly grey literature), plus her own observations from experience. What was most notable about Michelle's approach to the ASL classroom was her versatility. She did not adhere to or limit herself to any one language teaching approach and instead preferred to continuously update her materials based on new ideas she came across. She was often the only ASL teacher in the programs where she taught, and so she did not have an easily accessible circle of peers with whom to discuss teaching. Her mentors and informants were the teachers, authors, and thinkers with whose work she borrowed from.

Although Michelle used other people's materials as the basis for her teaching, she added her own flair to her lessons - most remarkably, through magic and play. She is herself a playful person, often slipping in jokes and ASL word play into our conversations. She is also a magician, largely self-taught. In one of our interviews she explained how one of her priorities in class is to encourage her students to relax and find joy in signing, which she says makes it easier for them to learn. One of the ways she accomplishes this is by teaching magic tricks. At one point she showed me a rope trick (which I failed at); later, a disappearing red foam ball trick (she gifted me the ball afterwards). Although I was in the interview as a researcher, a scholar trained in language teaching and learning, I was excited like a child when I attempted the tricks. It was not hard to imagine her classroom as a place full of laughter.

Another one of her methods was to integrate play-based learning. She demonstrated several of the games she has used in her courses. One was especially memorable. The game involved the students sitting around a single table covered in small toys - animals, toy cars, and so on. She instructed the students to choose one and then try 
to build up a collection by bartering with their classmates. She explained that students would get so caught up in trying to win that their stress about their limited language proficiency melted away. Thus, with limited formal training in second language acquisition or language curriculum, Michelle had developed her own theories of teaching and learning that had successful track records for her students and that reflected her own personality as a teacher. The piles of thank-you cards and glowing student evaluations that she kept in a box were some evidence of that.

Extending Michelle's experiences of developing her own personal collection of theories of language learning and unique teaching practices, the stories in the upcoming section expand on formal teacher training activities that ASL teacher-participants have undertaken. To reiterate (see also Chapter One and the Glossary), I use training to refer to formal professional development activities - where professional development is an umbrella term for formal and informal opportunities intended to support teacher learning — such as workshops, presentation, and credentialing programs (e.g., certificates, higher education degrees). I use training program for more extensive, usually multi-part, multi-day trainings like the trainings described in teachers' stories below.

\section{Teacher Training}

Not all teachers had yet participated in ASL teacher training, but four did and found it to be a formative experience. Donna had an extensive resumé of teaching and training experiences, mostly workshops and presentations, both as a participant and as a facilitator. Ken estimated he has done over 20,000 hours of workshops and presentations: "I went to everything! So many. Too many to count". Michelle and Erica both attended training programs early on, which helped build their skills and confidence to pursue their 
careers in teaching. In this section I focus on teachers' experiences in the training, but have appended several resources with further details about each.

\section{Donna: "I've been doing this a while"}

Donna estimated there were around two dozen ASL instructors in her province working in the various institutions (deaf schools, high school, universities and colleges, community programs). She reckoned she knew most of them from attending workshops and through her various responsibilities with the deaf cultural association:

That's my responsibility [with the association]. When I hear something, I pass along the message. If someone needs something, I put them in touch with whoever is best. That's how I know everyone. Sometimes they come help with workshops. It's a pretty good network. And I've been doing this a while.

Donna explained that the function of teacher training was not only the learning that happened at the workshops, presentations, and so on, but also "that's where we network, we hear other teachers' ideas, learn new ways to teach". She explained that many years ago she was part of several informal teachers' groups where teachers would "get together and keep in touch":

Over time some of the groups dissolved, but workshops would bring them together again. That's where people would share ideas and stuff, about curriculum, materials, whatever it was. It brought them back in touch. Sometimes people were invited from outside to learn more about what they did. 
She felt this kind of participation was especially important for younger, newer teachers who did not yet have much experience or opportunity to connect with their peers. She described in depth how she and her group of teachers recruited new people to teach:

A few teachers have emailed me to say they're interested in maybe becoming teachers, they'd like to try it out. So we'll set them up with a sort of mentorship period so they can see what it's like and if they want to keep going. Then they can work up to team teaching, see if they like that, and then on from there. One step at a time. Tutoring, more workshops, maybe a few volunteer teaching assistant jobs... there's different ways they can get into it.

People who would act as mentors would vary. We want experienced teachers obviously. I'd either put out a call, letting people know we're looking, or I'd reach out to a few people directly that I think might be good.

There's a few things I encourage them to do at first. If someone is interested they should always contact me first and I'll set them up with someone to get started on these things. I'll make sure their schedules work out with whoever I place them with. So like teaching in the evenings or only on Mondays, whatever.

I need to make sure the teachers know what they're talking about. Most of them- many of them didn't learn about ASL linguistics, for example, so I need to make sure they're ready to teach students. Let's say in high schools, teachers have a teaching degree, but in community teaching, they might not have a certificate or any training. We wanna make sure they have access to the training they need. 
In the Fall we'll have a meeting- and I'll know by tomorrow if it'll happen, [a colleague] and I will get together and discuss where to start, where to start with planning. What issues we've had, what ideas are going around... where to begin? We'll check with the community first, check with teachers. What are you struggling with, what questions do you need answered. And we'll develop the workshops from there. We're hoping those will come together by next year.

Donna's comments reflect McKee and Woodward's (2014) finding that sign language teacher professional development can provide opportunities to build and strengthen deaf communities. Based on my interviews with teachers and deaf cultural organization representatives, in Canada, deaf cultural associations are the primary organizers of ASL teacher professional development.

\section{Ken: UNB "Sign Language Instructor Training Program"}

Participation in ongoing professional development was required at the interpreting colleges where Ken worked in Western Canada. At the time, his partner, the hearing interpreter and his teacher-mentor, was well-connected to professional development opportunities and helped him find workshops and presentations. Ken was also inspired by his attendance at the 1989 Deaf Way conference, an international conference on deaf culture, where he met "loads of people":

The whole experience was amazing. Seeing everyone's different signing... I was blown away. When I got home, I was very motivated to keep going to workshops. Anytime there was a presentation or workshop, I would go. It took off from there. 
His list of workshop and presentation he has attended was four pages long, and he had an equally extensive list of workshops and presentations he has offered to others.

One of the more extensive workshops he participated in was a multi-day teacher training offered by the University of New Brunswick (UNB). The program was intensive, rigorous, which he liked. He recalled only a few specifics of the training since it was over thirty years ago:

It was a training program specifically for teachers using that book [Signing Naturally], that curriculum. The first and second levels. So yeah, it wasn't a general teaching degree; it was specifically teaching using Signing Naturally. The training program doesn't exist anymore, though. I went there in maybe... '87. It was a while ago. They had just established the program. There were two people from each province that joined the course and the idea was that we would learn and then go back to our province and teach other teachers what we learned in the workshop.

The [ ] course was only over the summer, maybe two weeks. That was it. It was very in-depth, very intensive. We covered a lot. Like, the first we learned was that each teacher is going to have their own way of teaching. We also learned about things like developing homework to give students extra practice. We learned what a lesson plan looks like and... there was more... Right. Like, how to use- how to develop our own teaching style when we're in the classroom, including things like classroom management.

Near the end we each had to teach with a lesson plan we developed and some of the experienced teachers would evaluate us and give us feedback on what we were doing well and what things needed improvement. 
That was the certificate! That was... that was every day for, I think about two weeks. Two weeks every day. We went through a lot of material.

I think we were maybe 20 or 25 deaf people, two from each province. It was a brand-new program and it only ran for that one year and that was it. After we did the course the program folded. That's it. It hasn't happened again.

There are several notable features of the program he described. One was the delivery model whereby participants attended with the expectation that they would share their learning with other teachers. This model was perhaps influenced by the financial costs of large-scale training, or perhaps informed by the perspective that teacher training is a way to enhance and empower deaf communities (e.g., McKee \& Woodward, 2014). Either way, Ken was grateful for the training opportunity and attributed the surge in his course enrolments to his implementation of the program's principles.

\section{Erica: CCSD "Unlocking Culture"}

Erica had found the Canadian Cultural Society of the Deaf (CCSD) to be "very supportive and encouraging of instructors who are in need". When she reached out about to the CCSD (i.e., the SLIC) about taking the ASLPI, they mentioned there was going to be an upcoming training program for ASL teachers. Erica remember being told it would be a training about using the Common European Framework of Reference for Languages (CEFR) in sign language instruction. The training was going to be led by a "researcher with their Ph.D." and "a man from Germany" and would be attended by 40 ASL and LSQ instructors from across Canada. Erica applied, was selected, and attended with her colleague not long before our third interview. 
When I asked about her experience at the training, she apologized and said that she was unable to share details with me:

The goal was to really discuss assessment - how do you plan assessments, how do you plan a course with assessments... There's a follow-up later. I can't share the information with you, but wow. It was wow. I'm so happy I could go.

The only other information she shared was that the follow-up was "to catch up and expand a bit on what we did in February, I'm really excited about that. It'll give me a chance to see how I've been able to integrate what I learned. That'll be the test'. I researched the program online and found some details about the program on the SLIC website (see Appendix K). I learned from the description that the training followed the same snowball model as Ken's, described above, where attendees were expected to share what they learned with other teachers. Erica's hesitation was therefore surprising, but a reminder that I was not recognized as a part of her community of practice. I did not push her for more information.

I wondered if perhaps Erica's secrecy was related to an incident with the SLIC that happened around the time of the training. In brief, a month or two prior to the training, I had contacted SLIC with a request to attend the training as an observer as part of this research. After delaying for several weeks, they responded that they could no longer support my research because Carleton had recently hired a hearing instructor and continued to act against the recommendations and values of the SLIC. It is possible that 
Erica was aware of this turn of events and hesitated to divulge details of the training for that reason. I reflect on the SLIC incident in Chapter Ten.

In any case, Erica had a great time at the training: "It was fantastic. It was... great. I met a whole bunch of new teachers, we could exchange ideas, lots of brainstorming... the presenters were phenomenal". The networking function of these training is no doubt especially valuable, as ASL teachers across Canada are very geographically dispersed.

\section{Michelle: CHS "Sociolinguistic Studies of American Sign Language"}

Before, during, and after our interviews, Michelle often asked me for ASL teaching-related resources and shared those that she had found with me in return. She always had her eyes open. She was a motivated and prolific researcher, finding articles, videos, and news related to ASL teaching all over the Internet. She also kept excellent records of her resources. During one interview she remembered that she had kept a manual from a training program she attended many years ago. She found the manual and offered it to me for my research. Like Ken, she recalled few details of it, as it had taken place over 25 years ago, but she gave me the manual. Without a good sense of her experience in the training, a full analysis of the manual was beyond the scope of the study. Still, I noted several of its features:

- The training program, called Sociolinguistic Studies of American Sign Language, was offered by the Canadian Hearing Society (CHS) in November 1991.

- The program had three parts: a pre-workshop, a core workshop, and a postworkshop, with a video-based exam at the end. Only the core workshop included live instruction that took place over a three-day weekend (Friday to Sunday). The 
pre- and post-workshop sessions were not instructed and instead required participants to watch videos, read, and review notes on the material.

- The required textbook was a popular series American Sign Language Green Books, A Teacher's Resource Text on Grammar and Culture by Baker-Shenk and Cokely (1991), or more commonly referred to as The Green Books for the colour of its cover. The selection of this textbook was likely on the basis of its authors being prominent sign linguists, which was the primary focus of the program.

- The program also aimed to prepare participants to use the "VISTA curriculum", i.e., Signing Naturally.

- The general rationale for the program was that whereas historically interpreters led ASL classes based on knowledge developed through their own training programs, now the deaf community is more active in teaching. This shift has caused problems ("problems" was underlined for emphasis in the manual) because the new deaf teachers have no formal training in ASL or teaching, and some do not yet recognize ASL as a language.

- The program manual's contents were a combination of lay summaries of ASL linguistic concepts, chapters from linguistics books, and extensive bibliographies of mostly ASL books and textbooks. Michelle had scratched some notes throughout but otherwise there was little evidence of her engagement with the material.

One recollection Michelle shared about her time in the program was that, despite a couple of teachers dropping out or not passing the final evaluation, it was an incredible experience for her to meet other ASL teachers. She described her cohort as a mix of 
people, all deaf, who came together and learned about their language, which, she reminded me, not all of them had previously thought of as a "real" language. She did not keep in touch with most of them afterwards, but they were her partners in learning for a time.

All in all, there were opportunities for ASL teachers to pursue professional development. Despite the SLIC having been largely inactive for many years, it recently (as of when the study was conducted in 2017-2018) revived its programming, including organizing the CEFR training that Erica so thoroughly enjoyed. The SLIC was also not the only source of training; Ken found ample training by tapping into the many resources available to interpreter educators through associations like local and provincial interpreters' associations (e.g., MAPSLI, AVLIC). The intensive training programs that Ken and Michelle attended were offered through institutions unrelated to the CCSD: Ken's training was offered through the joint efforts of the UNB and the New Brunswick Coordinating Council on Deafness (Harley, 1989); Michelle's training was offered through the Canadian Hearing Society (CHS), which operates independently from deaf cultural associations. That said, these trainings were fairly exclusive and brought to the teachers' attention through word of mouth and insider networks. Therefore, it is unclear to what extent training opportunities are widely accessible.

As has been implied previously, collaboration was core to many of the study's ASL teacher-participants' experiences, although those collaborations looked differently across teachers and led to both positive and negative outcomes. The final two sections of this chapter explore those experiences in more depth.

\section{Collaboration and Community: Successes}


Nick, Nathalie, and David all found success through their interactions with colleagues as they strove for alignment with their respective programs' philosophies. Nathalie and David's perspectives are shared here. Otherwise, Erica and Ken had both positive and negative experiences. For Erica the positive and negative experiences were closely intertwined and so I put her narrative into the following section about challenges in collaborations. Ken, however, had some positive experiences that were unrelated to some of his negative experiences, and so his narratives are spread across this and the following section.

\section{Nathalie "I try to match"}

Nathalie was of the mind that a good ASL teacher could sign and teach in any number of ways, so long as they recognized that they were part of the larger community of teachers in the program and reflected that in their methods. In her own teaching, she was energetic teacher with many ideas:

So many ideas for how to be better, try out different things. I don't wanna try too many different things at once, confuse all my students. [Laughs] And the next teacher will get them and be like, what did you teach them?? [Laughs] I was trying something new! [Laughs] Yeah I have a lot of ideas, but I don't wanna try the all at once.

She clearly valued innovation in teaching and was open-minded to different methods that might work better for her learners, but also wanted her teaching to keep in line with her colleagues and reflect the philosophy of the program.

She tapped into her program's philosophy by turning to her own past teachers whom she considered role models for her teaching for varying reasons. One teacher, for 
example, was easy-going and focused on gesture, where "as long as your movement is good, it's fine". Another was more focused on linguistics and "getting into the language and how different things make the meaning different, and focusing on how the language is built and broken up. I love it.". A third was introverted and understated in his signing, “He doesn't need to go overblown with the facial expressions or anything. You understand him. And even if you don't know the signs, he's still easy to understand. He's a great storyteller".

Nathalie also tapped into her program's philosophy through interactions with her current colleagues. Given she taught in the evenings she did not often have the opportunity for hallways chats. Still, she described a few practices that she had put in place to stay in tune with her colleagues:

And a few [of us share the same] office. Most of those teachers are first year teachers. So I try to match with second, third, and fourth teachers more. If there's a debate, a discussion about it, I tend to go OK, well, my students are going to be in your classes later. They might be in the first year teachers' classes. Not as important. Sometimes if there's like people with really strong opinions, OK, I'll consider it an acceptable variation. Not always. Like sometimes like hey hey hey hey. We're thinking completely different things. This sign, it means something completely different to you. Let's see what the Internet says. If we argue sometimes it's like, I don't know who's right. We'll use the Internet just to see what they say. 
Although she was a former student and thus intimately aware of several teachers' methods, she expressed wanting to do more collaborative learning, such as classroom observations, which she has done on occasion:

God yes! I looove going into other people's classes! ... I look at how their students are reacting, and also how they time things. So like if they do a lesson and a practice, how they do the timing, how they break up the timing. How they move from one thing to another. Yeah.

For Nathalie, her community and her priority for collaboration were her fellow ASL teachers.

\section{David: "What do I do?"}

Like Nathalie, David highly valued creating a sense of alignment, or what he refers to as "synergy", with his fellow teachers in his program. He described the discussions the group has about the importance of synergy:

Um.. well, [we had] meetings about how the courses are going to be taught in the future and, you know, creating a sense of synergy between the teachers... which is something that I feel very strongly about. Um... Because, for example, a[n ASL I] student will always, if they're gonna keep going, transfer into a[n ASL II] class. That [ASL II]class is going to be made up of [ASL I] students from all different classes. none of themsome of them might have the same teacher that they had in [ASL I], but likely the majority won't. Because of the mixing, right? So... how do you make a[n ASL II]- how do you- how do you set up [ASL I] so the [ASL II] teacher doesn't have to basically redo everything in [ASL I], or have to 
spend you know, two weeks figuring out where the gaps are and filling them in... which is sort of how it goes, right?

Since he taught in a university program, David explained how the program toed a fine line between prescriptivism through consistency across teachers and academic freedom that is inherent to university educators:

They're very- they're all university teachers, and this is, I think interesting [ ], because they're university teachers it's built into their minds that they have the right to teach a class however they want... Right? And so theythey're careful about, OK we need you to cover these basic things because they're needed for the next part. But how you teach is up to you. There's this careful- this edging around the issue, where it's not going to be like, This is how you do it and we're going to be checking. Right?... One of us will be in every one of your classrooms- No. No. They'd have a rebellion on their hands.

So what they're saying is, We need you- We have certain expectations, and the students need certain things to actually make sure they're learning the material. How you grade it, how you set up the marks for it, it's entirely up to you. It's your class. But the expectation is that they'll know certain things when they transfer. They'll need certain things when they transfer. And that's what they need.

And so... And that's part of the problem with teaching little isolated courses like [ASL I] or [ASL II]. You have to take it seriously, but you also have to let go of control because you have almost none. For me- So... ... It's this sort of weird place where you're sort of torn between your desire to teach them everything... and the reality that you can't teach them 
everything, it's impossible. And letting go of... whatever tensions come from that.

The tensions he referred to were the tensions between teachers' personal goals, methods, attitudes, and so on, and those of the other instructors and the program more broadly. David's story described the competing discourses, but he did not elaborate on how any conflict between them impacted him personally.

For David, there was an additional community identity that influenced his teaching work: his advocacy. His stance as a hearing ally in advocacy work about deaf issues (described in more depth in Chapter Eight) dictated that he respect the wishes of the deaf native signers in the program and in his local community:

I usually defer... because... I feel like, I'm a young teacher, just starting. I can teach at the university level because I'm so accustomed to university learning. But if I ever- if I was ever asked to teach at a- at high school level, I'd be like, What do I do? What do I do? Because I hated high school! So anything I do I'm going to immediately hate. So I'm gonna ask around, What do you do that your students like? What do you do that your students like? What- what- what's been going on, and I'm gonna do the best. ... So it's like, for me... the choices that I make have to be grounded in other people's opinions because it's not my place to make those choices. So I try to be very careful about the choices I make...

In another interview he described in tremendous depth his perspectives on linguistic descriptivism and the nested layers of impact of ASL teaching. In brief, he recognized that he was students' first point of contact to ASL as a language, their first linguistic 
model. As they would go on to higher levels, he would still be part of the tapestry of their language learning and use. Consequently, any language he used in the classroom was making language choices that his students in the future would carry with them into their own interactions with the deaf community. These future interactions would thus become part of the community language repertoire. In response, David attempted to make that flow of influence two-ways through deference to deaf signers in and out of his program. By integrating the language forms they approved, he was respecting that the ultimate impact of his learners on the signing community was theirs to shape, not his.

At the same time as David spoke of being an invested partner in the collaborative work of the teachers in his department and in supporting the wishes of the deaf community in his teaching, he described himself as "terrible at community":

I realized recently I'm terrible at community. It, it actually came up a few times recently. It's a- it's a-... it's a personality trait. I don't do large groups well. I've never been drawn to community in that way. My parents try to encourage me to be part of their community... and I just- I don't get the draw. ... With my parents it's [about family as a community]. Family is also a community, like they have that same thing. Now, I have relationships with individual relationships with my family and I'm trying to develop those relationships, but I don't feel a draw to family as a concept. ... And so... And- It's very different between my parents and myself. For them it's intrinsic. For me it's just like... wh-why? We don't really get like- It's not like I hate them, but we don't get along so like, what's the appeal? Right? For them it's deeply important.

Community for me is a different thing. Like, I used to go to socializing [at deaf events]. I used to go to socializing, while I was a student, I used to go 
every time. And I think I mentioned before, eventually I realized the reason I was dreading it was not, uh, the communication barrier because I was getting better and... that- it- I was actually not afraid of the communication barrier because I have no problem looking like an idiot. It's that I don't like being in groups. Th community- I wasn't getting something from being there as part of a community... In fact that was- it was unpleasant for me. I prefer one on one or a select group of people where we're all participating in a specific thing. Um... so it started there and I can see that it's continuing to a problem. I don't think in community terms.

When I asked him how he was defining "community", he responded:

For me the word community has this sense of... like an emotional tie... that I don't seem to have... So I just.... that feeling, I don't have that draw. I have trouble generating that draw for individuals, so community is I feel just beyond my reach. It's too abstract. I enjoy my time with this person so I will spend time like... But feeling an obligation to a group is... not something that I feel. But I feel the... lack of it, like, the, I see the- the advantage of having a community and I can see having those feelings as being the perfect of keeping that community alive... But I can't do it natively, instinctively. It just doesn't... you know.

And... that's a problem- I mean, it'll be a detriment to me on an individual level, but also because I can't inspire that notion in others. If I don't think about it myself. ... And I'm going to have to either accept that I'm just not going to do that, or learn how to do that, which is against my nature, which is a challenge.

In the end, he reflected on what he could offer his students: 
I have a grounding in- I've been a university ASL student. I've been through the program here. I've been through it when it was smallish. I've seen it grow. I've been part of its growth. And I can teach in that context. ... And I can touch on some of the complexity of the world outside, but I can't teach them to be community members because I'm not a community member. And I'm not even talking about not being accepted by the deaf community, I think I've gained some acceptance. But I'm not a community person. I can't teach something that I'm not able to do myself. Like, I'm just not that person. And that was something I realized consciously recently. But that's also, it's good to know that's not something I do well. And if I'm going to integrate that I'm going to have to find ways that work for me.

His assertions about being "terrible at community" are puzzling when put alongside his other discussions about how strongly he identifies with the common cause of not only his colleagues but also the deaf community more broadly. From the outside, he has found success with and within several communities. In hindsight, I could have pursued this apparent contradiction.

\section{Ken: "I'd reach out to anyone"}

Ken's entry into teaching was through a hearing friend who was teaching and mentored him, but as he has progressed through his teaching career, his local deaf community has been a major source of insight for his teaching:

What I would do is reach out to a bunch of deaf people and ask them to sign the sentences to see if they signed the same way as me. I would take the most common way and that's what I would put in the video. I would 
always reach out to the local deaf community to see what they did. It didn't really matter who it was. I'd reach out to anyone - old, young... didn't matter. Then I would compare across signers to figure out what they had in common and which ones were particular to the signer because if I started teaching one way of signing but it's only one person's way. It won't help. So, I had to be careful and make sure what I was teaching would be understandable to any signer who was watching.

This aligns with his goal for his teaching: to teach hearing learners-especially interpreters - to align with the local deaf. He went on to explain that he does not take the advice wholesale; he filters it:

The other thing is that some signers would want me to sign one way, but they don't have any experience with linguistics. But I do. I have that experience. I've studied sign linguistics. I know about syntax, structure, grammatical rules, past, present, etc. But most other deaf people just don't have this knowledge. They don't have this experience. It doesn't mean they're not smart; it just means they didn't learn it; it wasn't part of their education. So it was up to me to make sure the language I was teaching was accurate.

But it's funny because when I started out at age 21 I knew nothing nothing at all about linguistics, how to compare and learn from language... And, over time, I've picked up all of these things. Mostly on my own and from different teachers that I've had-especially my hearing partner, my ex teaching partner at the interpretation college. Every day he would explain things to me. I learned a lot from him... 
That was then. Now, I learn mostly through the Internet and with deaf friends. I chat with them about everyday stuff and film our conversations, and then I study it.

Ken described how he goes about involving the local community in his teaching and creating a network or community of teachers to build resources and capacity.

I compiled a manual, a guidebook of sorts, with these little steps to help teachers adapt the program for Canada. Plus, I created a series of two-hour workshops where teachers would come in and we would go back and forth and learn together how to use this curriculum until they were comfortable.

Eventually, he said, that group dissolved due to disagreements and differences, some of which I describe in the following section.

More recently, he started an ongoing collaborative effort to develop an MSL curriculum to bring into his province's interpreter program. The group currently comprises six people, a mix of genders and ages:

We sit around and name a whole bunch of signs and discuss how to sign them and their meanings. Then we narrow it down, refine the list to just a few pages. When we've gone through this process a bunch of times, eventually we'll have something that we can send off to be edited and published, you know, with nice graphics and layout. We also want to be able to have video links within the text where a series of videos that accompany the book so you can go back and forth... It's a big goal. 
For me, the most important thing is that we get a bunch of people together to brainstorm what goes into the curriculum. It's not just me working on this project. We have to work as a team, to get feedback from everybody to make sure it's local sign, the way that we sign here. The Gallaudet people can stay out of this. I think they'll just put us down, so forget about them. It would be a local project, for the people who grew up here. Some are teachers and some are just locals who have an interest in the language, but worked at hospitals or in construction or, you know, other kinds of jobs, so they have a good idea of the different types of everyday vocabulary. A lot of teachers have only worked as teachers so they might not know different signs for things. They won't have the first-hand experience with different types of everyday language.

He explained why it was important to him that it be a collaborative effort:

Some people feel really strongly about using only MSL signs and not changing to the newer signs, and others are more flexible. It really depends. That's why it's so good to have these conversations all together and spend a lot of time comparing signs and preferences... Part of what I'm doing is clarifying for people what is the older sign, the MSL sign, and what are some newer signs. It's really tricky. It's also a fine line sometimes... but I'm working on it. It's a lot of work. It's also really interesting and, honestly, it's really fun to watch and learn from each other.

His MSL group's work was essentially a multi-modal, crowd-sourced, descriptivist lexicography project. Although MSL has been used across the Maritime provinces for well over a century, Ken explained, it has largely blended with ASL. ASL came to the Maritimes recently enough that the current elderly deaf in the Maritimes use a blend of 
MSL and ASL, but anyone under the age of 40-50 is unlikely to know any MSL. Thus, there was a generational linguistic divide and a real possibility that MSL, of heritage significance, will be lost before long. Ken was passionate about preserving MSL, as his efforts suggest, by working collaboratively to bring it into the classroom.

Another notable observation about Ken's narrative above was his explicit exclusion of "the Gallaudet people", whom he considered to be nay-sayers and bullies. He eventually explained who he meant by "the Gallaudet people" and how they have caused conflict within his local sign language and deaf communities, as I share in the following section.

\section{Collaboration and Community: Challenges}

In this section, I continue Ken's above train of thought about rifts in the community caused by "the Gallaudet people". Following that, I share a brief narrative from Erica about the successes and conflicts she has faced with her own professional community, where disparities in formal teaching knowledge have made it more challenging to unite. I also share Donna's brief reflections on her story of struggles trying to grow and inspire the new generation of incoming teachers.

\section{Ken: "The Gallaudet people"}

In several stories so far, Ken has referred to "the Gallaudet people". For context, "Gallaudet" refers to Gallaudet University in Washington, D.C., a private university established for deaf and hard-of-hearing students. Gallaudet is a literal gathering place for deaf people from around the world to get a post-secondary education. On campus, ASL is the primary language of everyday interactions and even interior spaces were designed with visual communication in mind. Gallaudet is also a highly symbolic place of deaf 
collectivism, a defining feature of deaf culture, and of deaf empowerment through barrier-free education. In academic terms, Gallaudet University Press is a major publisher of countless academic and non-academic sign language and deaf studies books as well as Sign Language Studies and The American Annals of the Deaf, two top journals for deaf and sign languages scholarship, and the Deaf Studies Digital Journal, a video-based journal for a variety of deaf studies publications (scholarly, literary, artistic, etc.).

While in the common deaf vernacular Gallaudet represents a certain idealized space and place for deaf people, to Ken it represented a threat to his grassroots values and efforts. From his experiences, as described in the stories below, the ideas and practices that stemmed from attending Gallaudet or standing behind the people or ideas that did was incompatible to what he considered the Maritime way of doing things - that is, locally.

Ken's issues with the Gallaudet people extended across several experiences. He used to be part of both his local and provincial deaf cultural associations and said the groups started off with good intentions and good work, including a project focused on MSL documentation and revitalization. He described how in those days the organization used to gather together teachers to share ideas and knowledge about teaching, they offered workshops and training programs, and developed resources. Eventually, disagreements led to the cancellation of many of these projects and Ken was "kicked out" from the group:

The reason was that when it came to certain things, the Gallaudet people were more... discriminatory, saying certain things were wrong and all that. And I didn't feel that was right. The priority should have been the 
local community. This is a [local] grassroots community organization. It's not a fancy formal thing like the Gallaudet people wanted it to be. It should be all about here, but the Gallaudet people thought that was no good, low-level, not advanced enough. And things moved too slowly.

He relayed his disappointment that:

The community was looking at the Gallaudet people's ideas and they really seemed like good ideas, so they were in. They were convinced. I thought, we're not really ready for this. This is not how we do things here. I tried explaining this, but in the end, people had already formed cliques and ultimately most of them sided with the Gallaudet people. It was really awkward, a sticky situation...

Some of the "good ideas" that his peers endorsed included Gallaudet's "fancy materials and their standardized books". Ken had previously said his English reading and writing were not strong, which also referenced the social currency of these skills in the deaf community. Moreover, he felt that sign languages should be taught and learned through the signing modality, not through books and, as previously discussed, handouts full of writing. Thus, there was an immediate dissonance expressed between his views of good teaching and those expressed by Gallaudet and supported by his colleagues.

Ken's resistance to standardization appeared in another of his stories where he described a recent attempt by Gallaudet scholars to prescribe a "purified" version of ASL for the masses. I had previously heard of this initiative outside of this study and Donna and Michelle both referenced it as well in their interviews, both with skepticism. In brief, Ken's "Gallaudet people" were on board with a movement that proposed to reduce the 
amount of English influence on ASL by pushing signers to de-initialize signs-for example, instead of the sign for FAMILY having the "F" handshape, it would use a nonalphabetic or de-initialized handshape. Ironically, the idea was not from Gallaudet scholarship but rather a grassroots movement, promoted by signers in forums like Facebook (Kusters, Green, Moriarty, \& Snoddon, 2020). In any case, none of the teachers in this study who mentioned it were convinced it was a good direction.

Donna resisted the idea on practical and theoretical grounds: without an alphabetic handshape to distinguish initialized items such as FAMILY, ASSOCIATION, ORGANIZATION, DEPARTMENT, CLASS, and several other signs that are otherwise linguistically identical, signers will likely revert to using more exaggeration mouth shapes to clarify which meaning of the sign they mean. This compensation is, of course, simply trading reliance on one English system for another. She explained:

Like, FAMILY. I've seen lots of discussions about changing the F hand shape, de-initializing it. But I don't like that, I don't buy it. I like FAMILY and keep it like that. But the idea behind it is to purify ASL, to clean it up. But what you see instead is that when you lose the initial people just mouth more to say what they mean. You should really watch M.J. Bienvenu's [a prominent ASL linguist] video. She recently posted a vlog about ASL purification. It was really, really well said. I agree with her. I recommend you watch it. I see the debate going on between people about this, like with CULTURE. Once you take away the C, you need to mouth it, right? M.J. knows her linguistics, so I respect that. I added my thoughts, too. It's a whole big debate now in sign language. You should really watch the video. 
It's not real-world, what they're proposing. But that's how it needs to happen. You can't put theory to the side and expect it to work in real life. Also, like linguists showed that, like CULTURE de-initialized, you need to watch out because this handshape is associated with ABSENT, MISSING, PASSED AWAY. So the hand shape doesn't work. It's a conflict of meaning. It doesn't work in the real world. So the idea of purification is OK except it doesn't work in the real world. The theory and practice need to match. Otherwise people aren't going to use it, and it's a waste. M.J. gives a great analysis, she clarifies her reasons for not buying into this de-initialization, and gives examples... Hats off to her. She looked at the theory and then also what people actually do out in the community.

She then cautioned me to be careful about everything I see in vlogs ("Some people post a lot. But I don't really buy into all of it. A lot of them I don't know who they are. I don't know their background, I don't know their training, do they have a certificate, are they experienced? I don't know!"). She emphasized the need to find credible sources, to "do your homework" and investigate the person's background, "ask questions, be critical".

Ken disagreed with deinitialization on ideological grounds although several people in the community, "the Gallaudet people", were keen on the idea. For him, it was an attempt to control local signing. MSL had already been so thoroughly impacted and "invaded" by ASL that Ken wanted to avoid more forced change at all costs, "We needed to maintain our grassroots and stay within the community". He recognized that "the Gallaudet people's" principles did not nurture MSL or even ASL:

They ignore MSL. Even their ASL is more like PSE [Pidgin Signed English]. They use their fancy English words, their big technical terms, but they don't know how to sign for the community. I was really pushing 
for the community to consider that, especially since MSL is so precious here.

Ken resented that, despite all of his efforts to bring teachers together, to rally the community to support MSL, to improve interpreter education for deaf people in the Maritimes, the Gallaudet people made him feel diminished about his own skills and value as a teacher:

They had a tendency to rip people apart, including me. They really got me down for a while. They had a way of making me feel lesser about my ideas. They didn't really acknowledge that I have lots of years of experience in the community. I mean, they knew that I had lots of experience, but for their own reasons they were mesmerized and looked up to the Gallaudet people's ideas.

It was interesting because when I was in B.C., people looked up to me because I was an outsider and I had these years of experience that I brought with me. But when I got back home [ ] it was like all, Ken? Oh yeah, we know him, he's old news. They didn't value my experience as much. It's too bad, really.

Eventually, he left the association, but the rifts while he was with the association carried into his relationships with his fellow teachers and he even lost a few friends who chose "Gallaudet" over him. Ken was clearly bitter and disappointed about the lack of teamwork.

Ken's above depiction of Gallaudet not as a beacon of deaf unity and empowerment, but as a divider and a bully, was one of the most surprising narratives in 
this study. His responses here and earlier in the chapter to "the Gallaudet people" and their ideas pointed to how deeply embedded Gallaudet as a symbolic leader is in deaf communities. Their influence is less noticeable in ASL teaching, where Signing Naturally, to which Ken does not object, dominates. The dichotomous representation of Gallaudet highlights the layers of loyalties that sign language teaching can entail. His counternarrative is an example of individual resistance to a dominant discourse.

\section{Erica: "They're not interested"}

The final story in this chapter is Erica's about her experience trying to build a teaching community in her area. She explained that there were few teachers left in her Prairies province, and so I asked if they ever got together or if they mostly worked in isolation:

I would say both, because I bring more experience and education because of all the courses I took and the additional trainings. One other ASL instructor has taken the same courses and trainings as I have, and we can engage in discussions about our experiences and ways in which to improve our course delivery. Whereas the other two instructors do not have any formal training and I find it difficult to relate. They want to see our materials and how we conduct our classes and we are resistant to share because this came out of a great deal of work on our parts and if they were willing to pursue formal training, we could all work better together and more collaboratively.

There is a distinct disconnect with the instructors who aren't formally trained and a great collaboration with the other... I have tried to help and give them pointers, but it is not reciprocated simply due to their lack of understanding and education. Yes, I do value and appreciate them but 
strongly encourage them to pursue formal training and education to which they're not interested.

Erica's narrative explained a consequence of a variously trained community of professionals: stratification based on education and expertise. From her perspective, she was unwilling to open her resources to untrained teachers because she felt they would not offer much in return. Erica's hesitation to engage too deeply with novice teachers was a surprising counternarrative to what I had seen in not only her own early teaching story but also those of several of the other teachers; namely, it is common for experienced teachers to take novice teachers under their wing until the novices are ready to set out on their own. At the same time, given she was working no fewer than five part-time jobs at the time of our interviews, she likely wanted to value her time and energy. Nurturing relationships with teachers who were perhaps less experienced or even invested in teaching might have been perceived as an additional drain.

Lampert (2012) called for teaching to be more collaborative, what she referred to as a "generative dance". The idea, in brief, came from Hiebert and Morris' (2012) proposal that improving classroom practice should depend on improving teaching, not teachers. Lampert (2012) unpacked this idea by advocating for teaching as a collective, not individual, practice, or a "generative dance", which she described as the development and exchange of "knowledge embedded in artifacts and the individuals who learn how to use and continuously improve those artifacts" (p. 361). These artifacts were primarily curricular and assessment documents but could refer to professional development and other artifacts. The "dance" was specifically to counter the culture of isolation that can kill the technical culture so essential to well-formed professions, as I described in Chapter 
One. Lampert explicitly pointed to communities of teachers as being central to this process.

However, Erica's above story showed a less utopic reality: some teachers were simply more active, more prolific, better informed, better prepared, and perhaps more motivated to engage with the profession. These discrepancies caused some disjuncture between teachers. This finding was unsurprising given that in ASL teaching, as described in Chapter Six, new and inexperienced teachers rely significantly on their peers for support and mentorship, less so than on formal training activities. Erica—and Donna, as I describe below—-felt the weight of the additional task.

Before I present Donna's story, it is relevant to mention that one of the ASL program administrators I interviewed commented on how tensions between colleagues have appeared in the university setting where he worked as a result of having a large program with full-time as well as part-time teachers:

I think what happens is that when you have a big group of contract [parttime, term] instructors like that you end up with a lot of variety, and you have people who are determined, they have a real commitment to the program, despite their status, and really want to have a voice etc. etc. And uh, inevitably you have people who are, sort of, they've been sort of eroded, I guess you could say from years of part-time work. You know? I know- like, a lot of us have worked like that, too. And you're always on the outside looking in. You're expected to pull weight but there's no, sort of, real reward. So what happens is when you have a lot of contract instructors and some of them have been around for a long time, you get those types of dynamics. 
Although Erica was not in a large program or working full-time at her various institutions, it may be that she felt in a similarly broad sense as above that other ASL teachers were not "pulling their weight" while she was herself "eroded" from her own difficult and precarious work life.

\section{Donna: "It's a struggle to get people on board"}

Similarly, Donna expressed that in her province, there was ample money for teacher professional development, networking, resources, and so on. She was grateful for the opportunities, many of which she organized, participated in, and/or led. Yet, to her disappointment, some of her fellow teachers did not take advantage of the offerings:

Let's say for example somebody wanted to take up the SLIC exam. ... It takes a lot of encouragement let me tell you, it's tough. ... It's quite a struggle to get people on board. We've noticed that it's still the case that programs when they're looking for a teacher, many of the teachers who are being hired don't have any background and that's something we're trying to improve. I know that I've thought a lot about how to change things.

Donna was among the first teachers to get the ASLICE and considered herself a resource for younger teachers, "to guide them, give them pointers, to avoid confusion when they teach”. Like Erica, Donna's comments reflected how much she had been involved in developing herself as a teacher and how far she had come since the days when she was hired without any teaching experience. She had progressed to a mentor role, only to find that she expected more of the next generation of teachers.

\section{Threads from Chapter Seven}


The collection of stories in this chapter reflected the many ways that teachers have forged pathways through the profession, learning to align, reject, and modify the many narratives around "good" teaching, including collaboration. I have highlighted many key moments or threads throughout the stories and revisit them briefly here.

First, it is worthwhile noting that none of the teachers in this study referenced any of the formal standards of guidelines that have been established by ASL teachers' associations, scholars, and others (described in Chapter Three). Yet, many of the same points are reflected. Conversations around teacher standards, professional development, and qualifications are intertwined, as teachers put into action the beliefs and values about teaching that were imparted through their participation in various forms of professional development activities. There have been interesting discussions around all of these that help to illuminate some of the nuances of the teacher-participants' stories.

At the same time, as the teachers' stories in this chapter demonstrated, not all participants in the conversation about teaching as a profession were on the same page. Erica's later stories, for example, described how by her understanding, some of her less experienced colleagues took a less theoretical and less rigorous approach to their work. This caused her to distance herself from them rather than continue to mentor them in alignment with her values and practices. Donna also felt the weight of mentorship, although she and her community of teachers had developed a fairly structured system to maintain those mentorship relationships.

One of the strongest threads in this chapter in relation to divergent voices in the profession was Ken's narrative of "the Gallaudet people". His storytelling showed deep traces of incompatible teaching philosophies and teaching experience, and very different 
visions for pathways forward — although it was unclear if the final destination may have been the same for everyone. Ken's vision of the role of ASL and MSL teaching on the broader sociohistorical landscape were apparent in his narratives against "the Gallaudet people" and make even more sense in the context of his earlier narratives of the "good teacher". In those earlier narratives, he leaned toward teaching practices that were creative as well as ethically driven to improve the lives of deaf people (i.e., improved interpretation services, respect for deaf people). Thus, "the Gallaudet people" and their alternative theories and practices of the place of MSL and ASL were a threat to the knowledge he had developed in action that aligned with his visions for society moving forward.

David, Donna, Erica, and to some extent Nick, spoke to the same narrative as Ken: ASL teachers should teach with the goal of reducing misconceptions about ASL and fostering more respect for deaf people and deaf culture. This goal was articulated earlier in this dissertation (Chapter Three) as a common practice in ASL classrooms. This study's ASL teacher-participants all aligned with this goal, but told different stories about how their achieved it. Michelle and Ken, for example, showed ample creativity in their teaching methods - anything to respond to their learners' needs and meet ambitious learning goals. Erica and Donna were more inclined to use established teaching methods, such as Signing Naturally, adapting them based on experience and formal training. Nathalie and David, meanwhile, aimed to align with the practices of their colleagues, whom they portrayed as being the "keepers" of the curriculum.

Related to this, Nathalie and Nick were the only teacher-participants who spoke explicitly of allowing students to use their voices in class. These were interesting 
narratives in that they were told as secret stories, or stories that the tellers knew were in violation of the sacred story, in this case the grand narrative of the no-voice rule (described in Chapter Three). In the case of both Nathalie and Nick, allowing their students to voice, whether in class (Nick) or in office hours (Nathalie), was a practice that likely stemmed from their own personal theories of teaching and learning that valued ASL classrooms as comfortable and safe spaces, where speaking was a "safe zone" and perhaps a learning tool for hearing learners. Their personal theories drawn from practice took precedence over the common narrative about "good" teaching practice.

A final thread that appeared consistently across this chapter was one of growth. There is tremendous growth evidenced in teachers' stories, growth as they developed confidence in themselves as signers (e.g., Nathalie) and as teachers (everyone). Some growth came from independent learning, e.g., in-class experiences, and some came from collaborative engagement, e.g., in trainings, with colleagues, with the signing community. As the last collection of stories showed, the pathways toward growth was not without potholes and roadblocks, which took many forms—symbolic, human, and more. Still, and one of the intended contributions of this study, I hope that the act of teacherparticipants reflecting on the positive as well as the less positive aspects of their personal pathways was in itself a driver of growth.

Having presented a range of stories about teacher-participants' pathways through teaching in this chapter, the upcoming chapter, Chapter Eight, attend to teachers' stories about their pathways forward. 


\section{Chapter Eight: Pathways Forward}

The previous two chapters explored the early and ongoing stories about teachers' professional lives. This final chapter of findings extends Chapters Six and Seven by looking at teachers' reflections on their past experiences and their hopes and visions for their future experiences, or their pathways forward. For instance, where did they hope their pathways lead? How did they hope to shape others' pathways, e.g., their students and peers? How did they hope their pathways would shape the multiple linguistic, cultural, and professional landscapes in which they lived? The stories in this chapter explore these questions.

Each teacher had a unique vision of their pathway forward, where some were philosophical and others highly practical. Some spent little time on the topic and others had extensive stories to share about their pathways forward. Nick found life direction through teaching and saw it as a stepping stone in his plans to work as a politician. Nathalie focused mostly on the impact of teaching as a source of stability and community in her recently upended life. David had strong ideas about opening his students' eyes to deaf social issues. Donna was intending to fully retire soon and pull back from ASL teaching, but she also had big ambitions for her students' future as allies and supporters of deaf causes. Erica and Ken's stories feature heavily at the end of the chapter, as they were the most vocal about their vision for their teaching to create a better world. Michelle's voice does not appear in this chapter as she spoke little of her intentions for the future, except that she had grand plans to publish her extensive materials as a textbook.

I begin with Nick and Nathalie's stories, below. 


\section{Teaching for the Self}

Nick and Nathalie had both experienced significant changes in their personal lives around the time of our interviews. Nick had recently gone through a divorce and quit drugs, and Nathalie had recently learned that her hearing had deteriorated more and faster than she had anticipated. For both of them, ASL teaching represented a positive life direction.

\section{Nick: "This is like my new drug now"}

Nick had studied public policy in university and grew up surrounded by politicians. His goal was to work as a politician himself. I asked him how his political goal intersected with his current teaching work, to which he responded:

[My goal to work in politics] it's always on the side. Now, people are learning my name. Local people, but also, some of these people are from Toronto, Montreal... So one of the things I ask my students is, Where are you from? And they go, Oh I grew up in Toronto. Oh, I grew up in TroisRivières. I grew up in... uh... Vancouver. Or, I grew up in China, but I live here now. You know, they tell me that stuff, that's part of building- I'm trying. Tell your friends. And plus, you know, they always remember the deaf guy that can talk.

Nick was already very active and well-known in the deaf community, but teaching might be one way to build a voter base in the hearing community — or at least a reputation (as "the deaf guy that can talk"). 
As mentioned previously, Nick's desire to keep on teaching was not only a professional strategy; Nick has also found meaningfulness in teaching — and learning to teach—in a time of major life change:

I haven't taken any formal training yet. No workshops or presentations or whatever. But another teacher told me about a sign language teachers' something... I saw the website and was impressed. There was another website that she told about, too. I don't remember what it was, but I'm gonna be checking those out soon. She's given me a lot of tips, pushing me more- that way, towards teaching. And I'm following it. I wanna be here full-time! Like, I do wanna be a better teacher. I'm getting to be- a better teacher. It's gonna happen... It's just, I need the experience.

I do wanna make this, like, a career out of it. I don't want it to be like a one-off... I want it to be something that's lasting and... cuz like, I get a really good feeling after every class. And I wanna keep that going. You know? I used to do drugs, so this is like my new drug now. It's teaching them...

Honestly, my life kind of turned upside down two years ago. It's not really private exactly... My wife left me two years ago. Yeah it was two years ago... last week... She left me. Yeah, this past Friday. On the bright side, it's also the anniversary of being smoke-free! Yeah! The day my wife left me I decided to quit smoking. I told my doctor, I'm quitting today. He asked me why and I said, Well, my wife left me. And he was like, Oh... Okay... I quit drugs two years ago also. Yeah... Two years ago my life flipped completely upside down, right on its head. Or- it's like somebody hit the restart button on my life. And honestly ever since then it's been going really well. 
His story draws on a discourse of empowerment — not DEAF CAN; rather, I CAN. He started by reinforcing that he was a novice teacher and justifying that he had not yet undertaken any formal training, which implied he values it, by explaining that he was drawing on the resources he had at hand (his colleague) to do the best he could. He then reiterated, whether for his benefit or mine, that he was committed to being a "a better teacher", but that it would happen with time and experience. He may have been acknowledging the knowledge teachers gain through experience in the classroom, or he may have been emphasizing that he sees a future for himself that involves participation in professional development activities like "workshops or presentations".

\section{Nathalie: "Teaching is a definite 'Yes' in my life"}

Early in our first interview I asked Nathalie casually, almost in passing, if she was enjoying teaching so far and if she hoped to keep teaching after this term. I had expected an equally casual response like, "It's good, I like it". Instead, she paused, looked at me, took a deep breath, and explained just how loaded a question that was:

It is a very big question. Whew, how to do this, um... OK I'm going to have to go back a bit... So, I had a lot of big changes in my life, I recently got promoted, got married, bought a house... Saw an ENT [ear, nose, and throat doctor], an ear nose throat doctor... Um. ... I haven't had an ENT forever. It took about a year to get an appointment with an ENT after getting referred. Went to the ENT... Um... so... I keep telling myself that I've come to terms with my hearing loss... I haven't, I totally haven't. Let's just put that out there right now. Um...

[Before the ENT visit] I had very much thought, I love ASL... deaf community can be really great sometimes... I'm likely going to get deafer. 
When I do, I'll likely have to stop working at my government job. ASL teaching would be amazing, a dream job, best thing in the world. And I'll probably... Not to say that teaching isn't a full-time job, it just doesn't take as many hours away from home. So you'd be able to do more, like taking care of kids and stuff. So I'm thinking to myself, I'll semi-retire, take care of kids, live a great deaf life. And that was my plan. That was my goal. ... $\cdots$

I have no idea what I want anymore. My life is kind of in chaos! Part of me feels like I went, Life... [Gestured throwing something up into the air] Oh shit! Just threw my life up in the air, see where the pieces will fall. So. Interesting question. I don't know, is probably the most accurate answer. Yeah, I'd love to continue to teach, both, teach and do my other job. Um... I think right now that's probably the best decision for me. I've had a lot of changes happen recently and I don’t know how much more change I can deal with.

As she sat across from me, she was visibly struggling to wrap her head around everything as she spoke. Her speech was hesitant and full of pauses and she seemed to search for the right words and a direction for her story. Her speaking came out in a continuous stream, as implied by the absence of paragraph markers above. It was a powerful monologue of vulnerability and layers of restrained emotion around the new life she was now faced with. I have kept many of her more extensive stories intact over the course of this section because they were rich and deep narratives of the internal debates that she was living with.

She mentioned above that she knew she was headed toward a "great deaf life", but as she kept speaking it was clear she had not expected so much, so soon: 
I, I ... I'm deaf. I cannot have a one-on-one conversation with a hearing person unless they speak loudly and clearly all the time. Without asking them, sorry what was that? Can you repeat that for me? Uhh yeah can't get this at all. Can you write that down? ... It's a little frustrating.

I had to come to the conclusion that I was not going to get a cochlear implant. ... I had this appointment... ... [Takes several deep breaths] ... Umm, I realized I'd stopped listening to music for two years... I love music! Music is important to me! Oh my god I've been feeling so dead inside! I haven't been ASL-ing- [Laughs], ASL-ing... signing as much...

Um... And I'd stopped listening to music. So I stopped doing deaf things, I'd stopped doing hearing things. I wasn't doing things that were important to me and let life get in the way... When I listen to music now, I love it, and I hate it. Um.... When I listen to songs that I'm very familiar with, sometimes I can't follow along. And these are the songs I know inside out and like, I can't hear the music. And it kills me on the inside. It kills me! But... I've stopped talking to some people just cuz, well, it's hard to talk to everyone. Yeah, I know, sometimes just getting older some people fall away. It's just happening very quickly.

I knew this was going to happen, I just wasn't expecting it to be quite so soon. I was thinking maybe five years... But the ENT visit meant I had to face the reality of all these plans I had made. It was a lot to take in.

Nathalie put her past, present, and future side-by-side in her story. She described how she had stopped listening to music and could no longer carry on a spoken conversation unaccompanied by visual cues or signs alongside reflections on her current anxieties and 
decision-making. Through it all, her story was ultimately future-looking — and her future intimidated, even frightened her with its imminence.

She described how she had increased the frequency of her participation in deaf events, especially deaf sports, as a way of reclaiming control and joy in her life.

But now I'm playing deaf darts. I also just started ball hockey. I'm very excited about that. It's- it's really good. I didn't realize just how much I missed doing deaf things. For the past year, my sign exposure has been... not great. Not non-existent, but not great. More like in passing, signing, chatting, whatever. But I wasn't teaching and kind of lost touch with it. Plus I had a whole bunch of other things going on in life. I got really swept up in all that, and it was fine at the time.

Now that I'm out doing stuff with deaf people again, it's so good! Like, last week I started ball hockey. A friend was like, you should come! And I'm like, yeah I can do that. The first two times I tried going it was cancelled for crappy weather. And then last week I tried. I thought oh yeah, it'll be fun, cool to hang out with deaf people... Oh. My. God. I loved it so much more than I thought I would.

And I've never really played ball hockey! I don't know if it was a team thing, if it was a sports thing, if it was that I was hanging out with people I could actually communicate with... All of the above, maybe. I don't even know. I looooved it. I loved it! I can feel a world of difference now that I'm back. I feel alive again.

Her story displayed the joy of coming back to a space and a community where she could participate without her hearing being an issue. She felt the same way about teaching: 
So when I got the "Do you want to teach a class?" call, I was like, Yes!

Oh god, yes. It was one thing I was more certain of than anything else.

Yes. I wanted to teach. I love teaching. I love signing. Yes... Yes... I

missed it. I didn't realize how much I missed it. Like I was missing a little

piece of me, but I didn't know until I got that call and... I had it back. And

I don't think I ever really noticed that quite as much before this time around.

Teaching is a definite yes. It's a little bit of everything... It's definitely for myself. And I don't think I ever really noticed that quite as much before this time around. And um... But for myself on so many levels. I like... I'm very much an extrovert. Um... I grew up hard of hearing and my hearing's gotten worse over time. And... I find I'm feeling more and more isolated... And...... taking the hearing aids out and teaching sign language... like... it's great to be around people and communicating like that.

And yeah, it's partly the students. It's great to see the students... figure things out, and... just embrace the language and go with it. Uh and like every once in a while you get students who are crabby and arms crossed and like come ooooonnn... They all go through their own stuff... I honestly love my class this semester. Like, they're, there are a couple of trouble students, but for the most part, even they participate. For the most part the students are really engaged.

Coming full-circle, Nathalie eventually responded to my question of whether she saw a future for herself as an ASL teacher: yes. In taking me through the roller-coaster of change and anxiety that defined her life at the time, she showed a willingness to openly process her emotions with me. She did not at any point consult with me for my opinion or 
support, nor did I offer; she only needed to be heard and perhaps attempt to make sense of her possible pathways forward.

Nick and Nathalie's stories and motivations for teaching were a reminder of the humanity of teachers: human first, scars and all, and teacher, paid labourer, second. They lent perspective on their work as one aspect of their layered past, present, and future lives. They gave a lens on the profession that departed from the historically common discourse of teaching as a maternal or moral profession (Connell, 2009) where the client-learners, community, society, etc.-comes above all. They also contrasted the other teachers in the study whose stories presented in the next section were significantly more outward-facing; as in, they talked about the impact of their teaching in terms of how it affected others — students, deaf people, and broader society.

\section{Teaching for the Students}

Throughout the many of the teachers' stories presented in this dissertation so far, there have been many different expressions of their hopes and goals for students, including enjoyment, increasing their signing proficiency, and developing a respect for deaf people. One of the ASL program administrators I interviewed speculated that the success of their program was the thrill and the challenge of the new modality and new frame of mind needed to jump into sign language classes:

I think students really get delight from being able to grow as signers and be able to communicate in that totally different channel. And people, some people have deaf people in their family, they know deaf people. And there's also maybe an increased awareness of, of the deaf world, generally speaking. It's even percolating through popular culture, a little bit, now 
and then. And it, it just draws students for those reasons. Some might say it's because there's no reading or writing involved.

But there's other huge challenges in becoming a proficient signer, or going through the program. Taking an introductory course, any introductory language course is going to have, any language is difficult in its own way. I think ASL presents particular challenges. That thing about having to turn off your voice. And, yeah. It's tough.

He touched on one of the common myths that surrounds ASL, that ASL is easy (see Chapter Two), and that he was well aware that it was not. He was also aware of ASL classroom practices such as the no-voice policy. Perhaps most notably, he was aware of the potential for ASL to increase hearing people's awareness of "the deaf world". The teachers' stories in the following section point to student awareness as one of the main goals of their teaching.

David expressed sincere enjoyment of his teaching work, but he did not focus on how it fulfilled him as an individual; rather, he centered his stories on how he hoped his teaching would fulfill his students as individuals. Donna, Erica, and Ken talked about the impact they hoped to have on their learners, but in their cases learner impact was not the end goal. Instead, the changes they targeted were widespread institutional and social change, and so they are discussed separately in the next section. In a way, the same held true for David since his goal for his learners was to improve their awareness of audism and hearing privilege, which was intended to ultimately have a positive social impact. Still, David limited his scope of impact to the people and circumstances that were immediately within his current scope, i.e., the classroom. 


\section{David: "I want them left with questions"}

David was the only born hearing, raised hearing, and still full hearing teacher in this study. Unlike some of the orally-raised deaf/hard-of-hearing teachers, David did not straddle multiple worlds or juggle multiple identities. He also did not dwell on any discrimination or negative interactions he had or continued to face from teachers who, like Erica and Ken, believed firmly in deaf-only hiring for ASL classrooms. Instead, David reflected on his position as an ally to the deaf community and his responsibility as an educator to guide his students to and through the uncomfortable process of recognizing the pervasiveness of audism and hearing privilege in mainstream society. He also considered it his responsibility to help his students recognize the ethical responsibility they were accepting by learning ASL as the language of the deaf community; as he put it below, once you learn about deaf history, culture, and issues and once you learn ASL, "you don't get to not use it". His reflections on the topic were extensive and scattered across several interviews, and so I have placed several of them side by side below to give the full effect of his stance:

The big one for me, and it's the one I struggled with because I don't know how to fix it, is that because I'm not part of the group that is oppressed, I get to live my life never experiencing it. I've never seen a black person attacked, I've never seen a deaf person mocked. I know it happens. In my head I know it happens. I've seen videos, like I've seen videos out from the States and, I know these people who have horrible experiences and I know it's happening. But I've never experienced it. That's my privilege. Right? I get to live without having to deal with it. I can choose. I can choose to take it on, to learn, to understand, to change things up as best I can. But I don't have to do that... That's the basic privilege. 
Right now I'm teaching what I'm struggling with. Like even in the class today I said, You have to think for yourself on this. If you have a deaf child, what are you going to do? What if... what if you finish fourth year and you keep signing and you're socializing and it's great. Then you meet a partner and you get married. And then you have a child... But the partner doesn't learn ASL, the child is deaf. So what do you do? It's like, well obviously we're just going to teach them ASL, it's easy. But it's like, I don't know ASL. It's like, learn ASL! I learned. Well I don't wanna learn, it's too much time... What do you do there? I told them, There isn't one right answer. I said, luckily it's your last test, so you have time to think about it. ... But that's- I want them left with questions. I don't want them left with answers, because there isn't an answer. I prefer to leave the bias to them to figure out. To self-reflect. Especially in first year. Because as they go on, it's just going to get harder and harder. You can't ignore it at that point. You're going to have to be thinking about it.

Most of the [upper levels] are taught by native signers. Deaf people. ... That's, I feel, a good thing. It's not that a hearing person couldn't teach it, they absolutely could. But... you really have to know why you're doing what you're doing. And it's a shift of perspective. By that point they should be skilled enough- or starting to be skilled enough to really communicate with people. Then you can start being like, OK this is what it's like in the community. This is what you're getting into. I warn them at the end of first year. I say, If you're going to continue, here's my perspective. Right. At the end of [ASL II], it's like, If you continue on doing this, you are taking on a responsibility. To use it. You don't get to 
learn this and not use it. I don't mean you have to go out saving people... It just means, you don't get to choose to not sign with someone. If you see a signer, you have to use it.

I have to be careful of my advocacy because I can't overstep, but also my advocacy demands that I respect whatever community decisions the- the... native members make.

[ ] So, in the end, I really am teaching them to be ASL students. And that's a hard sort of place because... it's not enough. ... It's not enough.... because of my advocacy.... it's not enough... to just train them to be students. ... But at the same time, if I'm teaching a group of 30 [ASL I] students that I'm never going to see again, how much responsibility can I take? That- the responsibility, the decision has to be decided on at a faculty level, not at the individual level. [ ] Those kinds of decisions have to be done at the faculty level and they have to be done by members, native members.

In [ASL I] it's more like, Be prepared, this is going to get harder. And if you're not willing to take on the task, don't continue. Yeah. It's not gonna get easier from here. It's going to get harder. Cuz by [ASL II], they're already getting into, uh, perspectives on disability and that kinds of stuff, and so it's like, community aspects. Like medical, critical, social models of disability, that kind of thing... You don't get to learn it and not integrate it. It's your responsibility, now that you know this, you can't not act. No more claiming ignorance. You are no longer ignorant. Before, no one 
could blame you. You're trained by society, society is very ignorant.

You're not anymore. So you have the responsibility.

So it... That's the kind of thing for me... that's about being a [university] ASL student. That's where that responsibility comes from. It's not about... the community, it's about you are now an educated person, and you're not allowed to act in a way that is not education. You just don't have that option anymore! I can't force them, obviously, and they can just ignore everything I've said. But for me, if I left it unsaid, I wouldn't be doing what I'm doing. ... That's part of the advocacy. It's educating them not just in a language, but saying, You now owe something to all the hearing people who don't know what you know. To show them.

They don't owe it to the- I'm very clear about that, deaf people can take care of themselves. And, you know, if they've seen Gallaudet, if they've seen the Deaf President Now, they've been exposed to the notion that deaf people can do it on their own. They can take care of themselves. The hearing people are the ones that need help.... The deaf people, your only responsibility to them is not to ignore them. ... If you see a signer you say hello, if they're open to conversation, converse. That's your responsibility.

Everyone else, it's for- if you- you just can't ignore people saying stupid things about deaf people, you can't ignore people being ignorant about disability, you know... just... now you know. You have to act. ... I leave them with that message and then it's like.... And that's all the responsibility I can! Because I'm not going to be following them. I'm not gonna be, like, lurking over their shoulder being like, You know, that was an opportunity. To do something. I'm here judging you. I can't. 
Even at a glance it is clear that the recurrent themes in David's narratives were advocacy and privilege and the underlying discourse was one of equity and social justice rooted in self-awareness. In laying out his positioning, he was assertive about the bounds of his work as an ally, an advocate, and a teacher; namely, deaf people needed to make the big decisions. He also did not construct his story as one where deafness equaled disability; he took a firm stance that deaf people were a cultural and linguistic minority.

He did not seek approval or to convince me of his position, or give the sense that he was fundamentally uncertain about his path. At the same time, he did not hesitate to lay bare his struggles with finding the "edges" of his work, or his scope of practice, in practical terms (he taught only beginner-level students - how much do they need to know right away?) and in philosophical terms (i.e., bound by his advocacy to respect the decisions made by deaf colleagues and community members).

In terms of narrative form, David took for granted that I understood the significance of his references to Gallaudet (University) and the Deaf President Now movement (I did). While Ken referenced Gallaudet as an imposition (see Chapter Seven), a monolith that threatens local deaf communities that are not its replica, David referred to Gallaudet in its common portrayal: a symbol of deaf empowerment. Deaf President Now (or DPN) was a movement in the late 1980s where deaf people across the USA rallied to protest the instatement of yet another hearing president of Gallaudet. The protests were successful and a deaf person (I. King Jordan) was appointed. The events of DPN live on in the collective deaf memory as a symbol or grand narrative of deaf resistance, of DEAF CAN in the face of hearing hegemony. His reference to both of these symbols reinforced his dedication to not overstepping his role. 
David's vision for his students' pathway forward spoke to discussions in deaf studies about hearing privilege. His aim for his students to identify and confront their hearing privilege was ambitious, especially since he taught first-year students in a novoice classroom, making these ideas somewhat difficult to convey. Nonetheless, he directed his own professional pathway using his sense of ethical responsibility to open his students' eyes to ways they have been interacting with the world they had not previously realized. The final section below continue on this same thread of reflection on the potential impact of ASL teaching on broader society.

\section{Teaching for a Better World}

Like David's, Ken's visions of his pathway forward were also extensive, as he had plans for significant changes to interpreter education and ASL teacher communities in the Maritimes - in particular, to unite deaf Maritimers, not divide them as they have been. Donna's pathway forward, described later in this chapter, was tied to bring a greater deaf awareness to hearing learners, like David, with the additional impact of bringing hearing students closer to the deaf community as allies. Erica's pathway forward, also described later in this chapter, was ambitious and specific: she wanted to see big improvements to the ways that hearing and deaf people interacted and to the harmful policies and practices that currently governed deaf lives in the Prairies.

As I did with David's stories above, Ken, Donna, and Erica's stories below are presented in multiple pieces that I have largely left intact in order to retain the full impact of their telling. 


\section{Ken: "I want to see more humility, more respect, and cooperation"}

Ken's intended destination as a teacher was to help rebuild two professions that are closely connected: ASL interpretation and ASL teaching. His overall goal came from his strong DEAF CAN stance and his belief in the power of collaboration:

Looking back, I've been teaching for as long as I have because, well, the main reason is I want to see the deaf community work together with the interpreter community. I want to see them get along, truly collaborate, and be on the same page. That would be a huge success for me. Because I feel like if hearing people come in and try to take full control of sign language education, I think it won't work out at all. It's really important to keep the community involved, to have their input.

I'm not against hearing people at all, I just think that it's too common to be patronizing towards deaf people, pity them, have the attitude of ' $\mathrm{Oh}$ poor deaf people, deaf people can't can't can't...' But no, deaf people need to have their own power, control over their own language. So hearing people who are teaching and in a position of power should really pass it on to a deaf person, so they'll have the power. That's pretty much my goal. It's a big goal. I want to see more humility, more respect, and cooperation.

In another interview he reiterated:

One of the reasons I've been teaching this long is because there's a lot of work left to be done. Like, I teach to help people communicate better, like hearing staff who have deaf clients. How do they communicate? That's always been a focus. Plus, I really hate the idea of deaf people being pitied, and so, right, so it's a priority for me that hearing and deaf people 
see each other as equals. I need you, you need me. Not hearing people looking down on deaf people. Especially in the workplace. Deaf people shouldn't be reliant on hearing people for their work and being valued and so on. I think education is a big part of that.

Otherwise... I would love to see Canada be a more accessible place for everybody where, for example, you aren't born into this constant sort of... control. People trying to control you, push you into their world. It's so important to respect- I have my own ideas, my own worldview. Leave me to it. Don't tell me what's right and wrong for me; I can do that myself.

I like to think of us as we're all human, we all have a heart and a brain and our own perspective on the world. Let's see each other as same but different. Doesn't matter if you're hearing, deaf, blind, whatever... We should all talk to each other and see each other as equals, heart to heart. Right? That's why I keep teaching. Lots of work to be done.

For Ken, it was important for deaf people to have the "power in the classroom" and in a position of authority in regard to their own language. He saw the ASL classroom as a place where hearing people can come and see DEAF CAN in action. For Ken, the shift of power in the ASL classroom may have been an opportunity to open hearing learners' eyes to their hearing privilege and the audism and phonocentrism that otherwise dominates mainstream society, as David also aspired to do.

Ken's reflections were in line with the common "rule" in the ASL classroom that teachers will be culturally deaf, or at least in deaf-hearing teaching teams (Wilcox \& Wilcox, 1991). On the one hand, as alluded to in Chapter Six, deaf-only hiring significantly reduces the pool of potentially qualified candidates and may lead to the 
hiring of un-/under-qualified candidates. On the other hand, there are strong reasons for the practice that make it unlikely to change. Schornstein (2005) argued:

A teacher of ASL should be an active member of the local Deaf community as well as a member of the nearest Deaf club. Students will emulate the teacher's example and, through that interaction, learn to respect both the language and the people who use it. An ASL teacher should instruct the students as much from experience as from the book. And a teacher's participation in the Deaf club allows students entry into this new world. The teacher acts as a bridge into this new territory and helps students to be as comfortable as possible. (p. 406-7)

The implication is that ASL teachers play multiple roles—e.g., deaf spokesperson and role model, "bridge" to the deaf community, drivers of students' enculturation in the deaf community— that are best occupied by deaf individuals who most embody the deaf experience. This stance has been expressed explicitly and implicitly in the literature (e.g., Kelly, 2001) as well as anecdotally in the common knowledge.

One of the ASL program administrators I interviewed in this study supported this stance when he explained how his program was growing quickly and they were looking to hire more people, ideally deaf people:

I'll say it even on record, I think it's a really good idea that that person is deaf. But we'll see what the collective determines, you know? I think, because there are these issues of, ownership of ASL, I guess you could say, and um the fact that, um, those for whom ASL is, in a sense a first language or a native language, are- it's a disadvantaged group. And I think you, want to have a preponderance of representation on your team of 
people who feel that, live that, understand that. Because it's part and parcel of ASL, isn't it really. The language is not just something objective to be studied and bought and sold and so on. It actually belongs to some people. It is- it has its roots in a very distinct culture, which has been a minority, culture with, lots and lots of challenges. ... So it's good to have that.

The emphasis on teachers being deaf was notable, as it diverged from the non-native speaker teacher debate. In brief, there have been longstanding discussions in applied linguistics about the legitimacy of the non-native speaker teacher who, some argue, lack the natural intuition and therefore expertise in the language they are teaching. In the context of sign languages, the native speaker itself looks significantly different. Quer and Steinbach (2019) said it well: "[the] simple assumption [that the native speakers is the idea user] cannot be made for sign languages because of their highly idiosyncratic sociolinguistic settings and in particular their dominant acquisition patterns" (p. 2). For starters, over $90 \%$ of deaf children are born to hearing parents (Mitchell et al., 2006) and sign languages are rarely transmitted generationally from parents to children. Instead, deaf children who sign will often come to sign language later than their hearing peers to spoken language and their primary linguistic role models will be hearing second language signers (hearing parents, teachers of the deaf, interpreters, social workers, etc.; Quer \& Steinbach, 2019). In short, there are few truly native signers according to scholarly definitions of the native speaker.

In relation to ASL teaching, the argument that the ideal teacher will be deaf is parallel to, not an extension or rejection of, the non-native speaker teacher debates in other languages. The priority, as Ken said above, is that the teacher embody the deaf experience, not be the "ideal" native signer. Since the work itself is language teaching, 
there are of course proficiency expectations, but as described in Chapters Three and Seven, current standards reference SLPI levels, not native user status.

Returning to Ken's story, Ken's first target for the future of his work was sign language interpretation. Ken had spent years being the go-to person for deaf people's complaints with interpreters, what he considered to be a "broken system". He has also spent countless hours following up on complaints with the local deaf service groups and filling in the gaps for friends and others who need extra support:

I'm trying to help fix a broken system here. There's no reason to ignore all these deaf people complaining. But why is it only me complaining? People say, Oh Ken, he's such a good leader, he gets stuff done... But I want to change the system so people don't have to come to me. They should be able to get a response themselves, and not be ignored. I've told the executive director [of the deaf service centre] that things need to improve.

He proposed that the issue was partly that the people at deaf services were burnt out, working with limited resources and inefficient systems that leave them backed up and disorganized. Human resources - interpreters — were also in short supply:

It used to be a graduating class of 25 students and now it's down to 4 . Most of the time, and I think it's still like this, it's all women and like a single man. No, now there might actually be 2 in the program now. And the rest are women. A lot of people withdraw for reasons like, Oh they don't like my signing. Or... Others have told me they withdrew because they weren't allowed to socialize with the deaf community. That seemed to be a common rule and it's crazy. I don't know where it came from! I don't understand. Why would teachers not want their students to socialize 
with the deaf community? It's so important! You can't interpret otherwise. The rule goes, you can't socialize with the deaf community until after you graduate, then you're allowed. And sure, there might be good reasons to delay socializing, but those reasons need to be made clear to the students.

Ken dwelled briefly but intensely on the point of not encouraging interpreters to socialize with the local deaf community, which he considers to be an essential feature of interpretation programs, in his case more so than for other teachers because of the blended MSL/ASL environment.

Adding to the issue was that from his observations interpreter training programs were leaving gaps in building up interpreters' skillsets especially in medical and other specialized interpreting contexts where a skilled interpreter is vital. He related horror stories from doctors and dentists visits where an interpreter miscommunicated information and put deaf people in difficult and distressing situations. He described how mental and sexual health awareness was so important in the deaf community where information can be scarce, yet the interpreters facilitating information sessions lacked the skills to explain the complex aspects of these areas. He also recalled his own experiences at workshops and events where interpreters provided unacceptable service. One interpreter shamed him and his fellow deaf attendees for not knowing a sign variation that Ken said was used by the local community. Another interpreter continued to use her own sign variation even after Ken requested she use the variation the deaf group were familiar with. A third interpreter was annoyed when Ken interrupted her to clarify a sign midway through a presentation. His response: 
No, that's not how it works. I'm not going to sit through an entire presentation that doesn't make sense because I've missed one sign. Absolutely not. It was obviously an important one, too, so I'm not going to wait until the end. Like, would you like that, in reverse?

His teaching and advocacy work were thus intertwined: educate a stronger interpreter workforce for a stronger system in the future.

Ken's other target for improvement was the Maritimes ASL teacher community. He wanted interpreter educators to improve their overall approach to teaching to be more accountable to their students, the program, and the community the students were being sent out to serve. His difficult relationship with his former colleagues and the general philosophy of the program, described in Chapter Seven, came down to accountability:

I noticed the teachers teach without a clear blueprint, without outcomes for what they want. When I teach I give them a clear blueprint or map of the course so they know what to expect. That way, when they do their tests, they can look back at the blueprint and go, Oh yeah, this was in line with what we were learning. And sure, sometimes things go off-course, whatever, it happens. But you still need something to be accountable to. They don't do that at the college. I think it might be changing a little bit, changing from just handouts and lecturing to more hands-on learning. At least I hope so.

While he had issues with the practical components of the teaching in his former program, at the core of the problem with ASL teaching in the Maritimes, he said, is that the profession is full of divisions, secrecy, and closed-mindedness: 
Lately, I've noticed that the number of deaf people who want to become teachers is far fewer of them than before. It's a dwindling group. There aren't that many people left. Lots of people have left and moved elsewhere, like to other provinces, or they've moved on to different careers. Some of them started, but lost motivation or interest partway through and dropped it.

It's also a really strangely competitive group. There seems to be a lot of infighting and protectiveness about ideas. And the secrecy! It's happened a few times that hearing people have come up to me and told me that they've been teaching sign language. They have a group of hearing students that they've been teaching in secret. In secret! Then they ask me if I can train them how to teach. But they're hearing. Hearing and teaching ASL. I mean, they know that hearing shouldn't be teaching ASL. They know that... and they know they shouldn't be asking me to train them. Come on...

He described how he was once approached to teach an ASL course and found out later that a hearing person was hired in his place. When he approached the hearing person:

I couldn't believe it. This isn't right. I met up with the hearing person and right away they got all flustered, like, "I didn't know! I didn't do anything wrong!" I told them, "Make your excuses however you want. You should know better." Honestly, it seems like once they were offered some money they put on these blinders. They were offered a job and they were going to get paid and that was enough to make them happy and that was it. I met with them to explain why it wasn't right. Well, of course, that didn't go over well. It turned into this big thing and they shut me out... yadda yadda yadda... Whatever. It was political. They just didn't get it. If the students were worried about communicating with a deaf teacher, they should have 
asked the association to bring in an interpreter for a day to get used to it and then go from there. But they didn't do that. And it's not their fault. How were they to know? It was the association's responsibility. They really should know better.

Like Erica, Ken had noticed fewer and fewer deaf people were taking on teaching positions, which he seemed disappointed about. He was mostly frustrated that hearing teachers, who presumably knew the implications of their actions in relation to the grand narrative of deaf-only teachers, took teaching jobs anyway. He was frustrated that the hearing teachers were considered and hired in the first place, which points to another broken system for him. And finally, he was frustrated by the secrecy and duplicity of the hearing people, his potential deaf allies, who he felt were taking advantage of their hearing privilege by embedding themselves in signing work.

Hearing people were not the only barrier Ken perceived to a stronger ASL teaching profession. He also felt strongly about improvements to the supports and structures for ASL teacher development, such as training and certification programs provided by deaf cultural associations and the SLIC. He described how with his province's deaf cultural association was "closed-off", where it was "the same small group of people sharing ideas only amongst themselves". When it came to supporting teachers, there were fundraising efforts to pay for workshop facilitators and so on, but:

Thing is, they keep the money for themselves to go off and do whatever training. They never share the money and they don't share the training. Like, they don't come back and have a workshop to follow up on the 
training, to share with others. They've never done. Never. And that rubs me the wrong way.

Ken placed high importance on collaboration, particularly among deaf people, and so his time working with the association and looking to them for support came to an end when he realized they were not meeting his needs.

His early attempt to get ASLICE-certified was also unsuccessful based on what he described as conflicting views on teaching methods:

I submitted my application for ASLICE many years ago. I remember I sent in my application, sent it off, and waited to hear back. When I did, the feedback I got was that everything was weak, but I passed linguistics. I did well on the linguistics part, so that was good. But all of the other things, like teaching path, or planning, I failed! It was really confusing. And I couldn't figure it out.

Their perspective on teaching was very different from mine, I think, because my partner who's hearing, he took a look and completely disagreed with them. He said, This can't be right! This is not a good evaluation. It doesn't make any sense! They're evaluating you based on a different style of teaching. All of the different aspects they were supposed to be evaluating, like lesson planning, what does it look like, what's it for, and so on- well, nobody taught me how to do these things. I learned and developed them on my own, in my own style.

At the time I wasn't sure what they were looking for, but now looking back I understand better. They were looking for a clear breakdown of activities, how long will they'll take, why you're doing them, how you put them together into a lesson plan to guide you in class... All these things 
were things I didn't know, I didn't really understand. I'd just been doing things my own way. I figured out a system that made sense to me and I think It was pretty effective.

So the feedback I got that I was weak in all these things, it made sense in a way. I mean, that was based on their idea of teaching. At the same time, what they were evaluating was relevant to teachers who were working in college and university and formal classes for professional programs. I didn't need these skills in my own consulting business. I already had a good grasp of how to move forward. It was my rules, my way. If they don't like it, fine.

I mean, the teachers doing the ASLICE don't have training in evaluation. They don't. I noticed they give the certificate to pretty much anyone, so it's not really training, it's more like... favoritism. It doesn't really make sense. I decided that I wasn't going to bother with that again.

I think that's why ASLICE is starting to crumble. I've asked many other deaf people if they've done it and they all said no, they can't be bothered. They'd rather work independently. If you notice, all of the people that have the certificate are from Gallaudet University. The rest of us are left out in the cold. So they obviously prefer that teachers all have the same style as they do. But that doesn't work for everyone. For people like me who have years of experience and learn to teach in our own way we are better teachers than the Gallaudet "teachers", if you can call them that, who went to university but don't have much experience. That's how I feel.

Once again, Ken's mentor, his hearing partner, lent perspective and support to Ken's teaching pathway. After his experience with ASLICE, Ken's issue was that it attempted to prescribe the "right" way to teach based on "the Gallaudet people's" philosophy of 
teaching, which clashed with his own on many levels (see Chapter Seven). Even though he understood and acknowledged the value of some of the feedback and requirements of the certificate, such as course planning, he held his ground: their way was not the only way. He was firm throughout our interviews that teachers should be open, flexible, and, as much as possible, collaborative. He had started his own teachers' groups many times in the past and at the time of our interviews he was gathering a new group together (see Chapter Seven). Their focus: develop an MSL/ASL curriculum. Ken's dedication to his ideals was pervasive in all of his stories.

\section{Donna: "We're teaching the language, but it's more than that"}

Donna's goals for her ASL teaching were likewise informed by her personal ethics, particularly around the task of guiding hearing teachers to improving their awareness of sign language and deaf issues. She described how many years prior, teachers in deaf schools were mostly deaf, "but over time there were fewer and fewer". Deaf education became dominated by hearing people and not all of them were adequate sign language models for deaf children. More recently, with the upswing in mainstreaming of deaf students, interpreters have become the main language models for deaf children. Donna took on the task of raising awareness about deaf issues early on with her ASL learners to ensure their language learning was contextualized:

When I was teaching in the university, we debated audism.

Discrimination. Oppression. And a lot of students were shocked. They hadn't thought about things from that perspective. They assumed things were fine. But when I explained about the attitudes of many parents with deaf children, they couldn't believe it. The facts are really shocking. 
I did this with them because, I mean, we're teaching the language but it's more than that, right? It's more than that. If I can change my students' views, their attitudes, maybe others will see that. There's power there. The more people the better to join the cause, to solve problems. Hopefully, yeah, hopefully teaching hearing students will bring more voices to the debate. People will pay more attention to some of these ongoing issues. That's why I teach. I'm teaching the language and a little bit about the culture.

Her aims are in line with scholarly discussions of sign language teaching. In contrast to commonly taught (spoken) language programs, where the language and culture are taken for granted as valid, ASL programs tend to take a languaculture perspective, i.e., where language and culture are inseparable (Kramsch, 1993), and so deaf history, culture, and advocacy issues are introduced early on. As Calton (2013) noted:

ASL instructors spend considerable time explaining the Deaf position and its implications for how students should respectfully interact with Deaf people, how the students should treat their hypothetical deaf children, and, in general, what rights Deaf people want for themselves. In doing so, they take an explicit political stance. (p. 119)

As Ken described earlier, deaf people teaching sign language is a form of deaf empowerment, of DEAF CAN, and a way to maintain deaf control over their language, which has long been — and continues to be in so many ways — controlled by a hearing majority. 
In real terms, Donna described how she brought current events into the classroom, such as the September 2018 rally for national recognition of sign languages in Canada (see Chapter Two for details on Bill C-81):

The rally [is] about reducing audism, reducing barriers and oppression. I showed a video about audism in class and we discussed it. There were interpreters there to get more people involved. Lots of discussion came out of that. And after the discussion, everyone wrote a reflection about the movie and the discussion. And so many of them were shocked. They had no idea about so many of the issues around sign language.

I think it was helpful for people to see that for parents who make the decision not to sign with their deaf child it's because that's what medical professionals have told them. They don't really have access to other information. They don't know all the facts. They're pushed in one direction and that's it. So that was a big thing.

Here, Donna referenced the issues Erica raises below: the dominant discourses around deaf people are still overwhelmingly informed by the medical model of deafness, where being deaf is portrayed as a deficiency to be corrected, not as a difference that can be supported through sign language education.

She extended her lessons on medical models and audism with her upper-level students who had by then more understanding of issues and the signing skills to engage more deeply with each other and the topic:

We would go further, like, what are your thoughts on oppression... solutions? Someone said parents of deaf children need better access to 
information, we need better signing rights, we need better education in schools... And all that is great, but what are we doing? It's a lot of talk, but no action, right? It's been so long of talk and that's good that more people are in the conversation, but it's action time. That's what the rally is about. We're hoping the rally will make things happen. ... We want to spark more productive debate to take action on things like audism!

Donna explained that she herself had spent so many years working at deaf schools in a signing environment, working alongside interpreters and other deaf teachers, that she had easy access to information and collaborations around deaf issues and movements, but many deaf people do not.

\section{Erica: "It's my language. It's precious to me."}

Like Ken, Erica wanted to see big changes moving forward. Erica explained that in her province, when a deaf baby is born, the hospitals direct parents to a pediatric rehabilitation centre staffed by audiologists, hearing aid and cochlear implant specialists, and speech-language pathologists. Deaf groups and service providers that support early sign language intervention are granted only limited access:

Sign language is completely forbidden there [at the rehabilitation centre], they don't support sign language at all. It's not that it's expensive or whatever, they just don't want it. They offer financial aid for the services they provide, but they won't fund sign language classes. So that leaves a lot of parents stuck, which is awful. To be honest, it's really bad here. 
Manualists in her province were up against policies that do not recognize the value of ASL to deaf children's cognitive and social development or to the broader mainstream hearing world.

Erica clarified that she, referring to herself and the many advocates she works alongside, was not fighting for all parents to reject assistive hearing technologies and choose sign language, only that parents be presented with the full range of options, including sign language:

What I want to see happen are some really big changes. Most importantly, parents of deaf children need to know their options. Not just learning about one way, with hearing aids and CIs [cochlear implants] and speech therapy... They need better access to options so they can judge for themselves what will be the best option for them. It's not at all neutral as it is. And we would want to be neutral. We don't want to push parents in the other direction either. But options and access to information is really important. Give them more facts to work with. And [the rehabilitation centre] only offers them one option. It's pretty sad.

The limits to sign language education have dwindled the province's deaf school and may be responsible for the low interest in ASL courses. She suspected that the drastic decline in the number of ASL teachers - from twenty to three at the time of our final interviewwas in part because of the negative attitudes and lack of support for ASL in the province.

Erica saw several solutions to the issue. One was through advocacy work, which she did at the local up to national legislative level. Another way was through awareness campaigns, including sign language teaching, to foster more positive attitudes about sign languages. She saw the potential to combine and leverage the success of both of those: 
The [provincial deaf cultural association] was recently resurrected and they instated a few ASL classes; there were something like 25 students in the Fall. And now, starting as of very recently, just a few days ago, ASL started again. It was a big class, which is great. But the problem is that the ASL teacher from before, there were three of us, and now we're down to two. [In another city], meanwhile, they had three, then two, and now one.

So... yeah... It's really not great. We're pretty tight now and have to be careful about the courses because there's a shortage of teachers. We're trying to focus on the CEFR training to see if that'll be successful, and then once that's moving, we'll deal with the teacher shortage. It's definitely a goal to bring in more young teachers, to get young people teaching. But it's hard! We found one young person who was interested. They'll start volunteering just to see if they like it and we'll see if they stay.

But this fall, what I think happened this fall was that the deaf and hard-ofhearing students put together an art show [ ] at the library about deaf life. So a lot of people saw it and witnessed sign language through deaf eyes. I think that generated a lot of interest for the Fall courses. Now we're up to... Um, I have 15 in my class. That's L2. In L1 I have 24 or so, $24,25 \ldots$ It's good. It's good anyway.

On that positive note, at the end of our final interview, I asked Erica why she continued to teach ASL:

Why do I keep teaching? I enjoy it so much. It's my language. It's precious to me. I like to help people communicate with each other. 
I was surprised that her immediate response focused mostly on language use given her deaf advocacy work. It was only later in analysis that I saw that those short phrases were in fact quite rich pieces of insight into her pathway. For instance, her reference to ASL as "my language" reinforced that her first language, (Signed) English, was not the one she identified with anymore. Also, in referring to ASL as "precious" she aligned with the grand narratives about what constitutes a culturally deaf identity, which includes valuing ASL as a defining feature.

I thought that was the end of her reflections and so began to wrap up our conversation. At that point she offered the following anecdote about a memorable communication breakdown she experienced as a way of expanding on her point above:

My ex-boyfriend and I were out on a motorcycle ride, him up front, me sitting on the seat behind him. And then a moose crossed the road. My boyfriend was driving carefully and he saw it in time, so he slowed down and the moose crossed and it was all good. But then when the boyfriend accelerated again, he didn't see there was a second moose and so it ran right into us, into me. The moose actually died, it was really bad.

So both of us were thrown from the bike and the ambulances had to come. But when they arrived we couldn't communicate. Right? I was sure I'd broken my leg, I couldn't feel it. And so I was pointing at my leg and shaking my head, trying to communicate with them. But they didn't understand. It was terrifying. They just hurried up and put me on the gurney and into the back of the ambulance. 
And that was awful too, because they had to strap me in once we were in the ambulance. I couldn't use my hands either. It was awful, really awful. So we couldn't communicate with the cops back when I was a teenager, but then I also couldn't communicate with the paramedics after the accident.

It's so important to be able to communicate with the people around you because you never know when you're going to need it. I always keep those stories in the back of my mind. That's my main motivation, that's what keeps me going. I want to have a broader pool of people that we can connect with, help and support each other. Those events are the ones I keep with me.

The image of her hands being physically and metaphorically tied in the ambulance - the effective equivalent of a gag to a hearing speaker — with the uncomprehending medics was poignant. Although she was reflecting on the incident from a practical perspective of the importance of accessible services for deaf people, the image of bound hands helped me to make sense of her earlier stories about the state of deaf educational policies in her province that are strongly DEAF CAN'T and how she hoped her teaching and advocacy would have an effect on changing that discourse to DEAF CAN.

In closing, the ASL teachers who participated in this dissertation study had a range of visions for their pathways forward in the profession. For Nick and Nathalie, the visions were more inward-facing, or how they envisioned their own careers and lives revolving around ASL teaching. For Ken, Donna, and Erica, the focus was on the difference they could make on their students for long term social impact. The final 
section of this chapter, below, draws out some of threads that appeared across teachers' stories in this chapter.

\section{Threads from Chapter Eight}

The above collection of stories reflected the many ways that teachers have forged pathways forward in the profession. As in Chapters Six and Seven, I attempted to draw out some of the key threads in the stories as they unfolded, and I extend some of the recurrent threads in this section.

One thread that emerged was the grand narrative and common practice of native signer teachers. Several of the teachers in this study were second language signers, and yet other than their language background, for the most part they shared common visions with the native signers for themselves as professionals and for their students as socially aware people. Based on the stories in this chapter, I cannot ethically or empirically take a stance on this issue, only that hearing status and native signing status seem to be on a continuum as I described in Chapter One. Moreover, the goal of increasing hearing learners' awareness, respect for, and ability to communicate with deaf people seemed to be a fairly universal aim. Whether that is enough to unite a workforce was unclear. The stories in this chapter suggest that lines will continue to be drawn around who can pursue

that goal, which again reflects the sociohistorical context of the suppression of ASL and deaf culture and ongoing deaf discrimination.

On a related topic, Ken's stories that picked up on his reflections in Chapter Seven about "good" teaching raised questions about the extent to which common teaching practices were in fact common. As I was assembling these stories, I mentally put the teachers in a room together and envisioned their conversations (in hindsight, this 
would have made an incredibly rich presentation of findings). I wondered how Michelle, Ken, and Erica would respond to each other's methods: Erica seemed to be consistent with literature about ASL teachers' use of the Signing Naturally series (see Chapter Three); Ken used Signing Naturally as a starting point but seemed to adapt it significantly depending on the context; and Michelle, well, Michelle used magic and toys! How would Ken and Donna talk about the ASLICE, which Donna had completed very early on and which Ken had found reflective of practices and grand narratives about teaching that did not align with his own? What would any of the teachers say about Nathalie and Nick allowing their students to use their voices in class, or about David being hearing? These were all questions that arose from the stories in this chapter and that would be worthwhile exploring further, even conceptually, in another iteration of this study.

A final thread that was carried throughout this chapter, and which I alluded to above, was the narrative of ASL teaching as having the potential to shift the broader social narratives about sign languages and, especially, deaf people. Although Michelle, Nathalie, and Nick's stories revolved less explicitly around this thread, they nonetheless explored it through their reflections on their teaching methods and personal theories about sign language teaching and learning. The other four participants were very explicit about speaking to this narrative and showed many variations on how it informed their day to day professional practices.

In the next chapter, Chapter Nine, I summarize the stories and the threads that emerged throughout Chapters Six to Eight, and pull them together into a discussion with the theoretical and empirical literature that informed this study. 


\section{Chapter Nine: Pulling the Threads Together}

In the previous three chapters I presented a series of stories told by this study's ASL teacher-participants. In their stories, they recounted and reflected on their experiences along their pathways to, through, and forward in the profession of ASL teaching to hearing adult learners. At the end of each chapter, I provided a brief discussion of the patterns or threads from the chapter's stories and connected them to the theoretical and empirical literature that informed this study. In this chapter, Chapter Nine, I pull together the threads in order to provide the reader with a final synthesis of the study's findings in relation to the research questions and intended aims of this work. As a reminder, the research questions were:

1. What are ASL teachers' pathways to the profession?

2. What are their pathways through the profession?

3. What are their pathways forward in the profession?

This chapter begins with a summary of the stories that this study's ASL teacherparticipants shared about their pathways (Chapters Six to Eight). The summary was intended to remind the reader about the incredible number and diversity of stories that were told in the teachers' own voices. Following the summary, I bring together the threads that emerged throughout Chapters Six to Eight in order to speak to the theories and literature that informed this study. Finally, I reflect on the contributions of this study in relation to the intended contributions described in Chapter One.

\section{Summary of Stories From Chapters Six to Eight}

The main findings from this study were the stories from the seven ASL teacherparticipants about their pathways to, through, and forward in the profession. In the 
sections that follow, I provide a high-level summary of those stories by chapter, beginning with Chapter Six, below.

\section{Summary of Chapter Six}

The stories in Chapter Six focused on the ASL teacher-participants' pathways to ASL teaching. I clustered the stories around teachers' first learning experiences with ASL and the deaf community, their entry into ASL teaching to hearing adult learners, and their early days of teaching. For ease of reading, I summarized the stories in point form.

- ASL teacher-participants had diverse backgrounds with ASL. Only one teacher was a native ASL user, two were native signers (MSL and Sign English), and the remaining four had learned ASL as a second language as young adults.

- Learning ASL had a profound impact on teachers' lives, particularly for those who learned ASL as a second language. For example, as Nathalie came to grips with her rapidly deteriorating hearing, she spent more time with ASL and the deaf community, which gave her access to a new social sphere where her hearing did not interfere with meaningful social interaction. For Michelle and David, learning ASL was eye-opening, as they had had very little prior contact with ASL or deaf culture.

- ASL teaching was an unexpected, unplanned career path. With few exceptions, the teacher-participants began teaching "out of the blue", where most of them were invited into the classroom by a teacher who mentored them for a time before passing on their teaching duties. Despite most of the teacherparticipants having other career plans prior to teaching, all of them expressed a devotion to their work currently. 
- Mentorship was a main source of early teacher learning. Since many of the teachers arrived to the classroom with little prior teaching experience or formal teacher training, their early mentors (usually the teacher who brought them into the classroom in the first place) played a central role in guiding teachers' initial development. Mentorship looked different across the stories-e.g., co-teaching, observation and feedback, co-developing and sharing materials, and "hallway chats".

- ASL teaching was a source of empowerment, where several teacher-participants described their early experiences as an ASL teacher as reinforcing their confidence as signers and in the legitimacy of ASL as a unique language distinct from English.

Next, I describe the stories from Chapter Seven that focused on the teacherparticipants' ongoing, everyday teaching experiences, or what I referred to as their pathways through the profession.

\section{Summary of Chapter Seven}

The stories told in Chapter Seven were more extensive and covered a wider range of topics than those in Chapter Six, including: perceptions of what it meant to be a "good" and an "imperfect" teacher, both in relation to themselves and to other teachers; classroom experiences, such as teaching materials and activities; participation in formal professional development activities (i.e., teacher training); and the many successes and challenges of collaborative work with other ASL teachers. The point form list below provide a more detailed summary. 
- The "good" ASL teacher meant many things. For Nick and Nathalie, a "good" teacher created a safe, nurturing, and motivational environment for new hearing learners for whom ASL was a radically different learning environment. For Erica, David, and Donna, the "good" teacher was well-equipped to prepare their learners to engage respectfully with the deaf community once they left the classroom. For Ken and Michelle, the "good" teacher was dedicated to constant improvement in their craft and maintained high professional standards for themselves as well as for their learners (in his case, future interpreters).

- Teachers saw themselves as "works-in-progress". Both the novice and experienced teachers actively sought opportunities for growth through informal (e.g., reflection) and formal (training, certification, etc.) professional development.

- Teachers used a variety of classroom teaching methods. In contrast to the empirical literature that suggested that the vast majority of teachers used the everpopular Signing Naturally textbook series (see Chapter Three), only three of the teacher-participants in this study used it - and even then, they adapted and supplemented it with other materials to suit their particular teaching contexts. The other four teacher-participants used a range of materials and approaches, including games and magic.

- Yet, some classroom methods were universal, such as the no-voice rule (see Chapter Two), which all of the teacher-participants described as being a staple of their classrooms. 
- Professional isolation and collaboration were both common. Nathalie, Nick, and Michelle described little interaction with other teachers, primarily because of logistical reasons such as teaching in the evenings when few teachers were around. The other four teachers described collaborative relationships with fellow ASL teachers to guide their teaching practice, some of which were productive and others fraught (see next point).

- Some collaborations were challenging. The ASL teaching community and deaf communities are by no means homogenous and members may sometimes hold opposing views. For example, although many of this study's ASL teacherparticipants were mentored when they first started teaching, they expressed difficulty in developing productive mentorship-based relationships with incoming teachers. For Ken, the issues with collaborations ran deeper, as he faced recurrent issues with "the Gallaudet people", referring to his peers in the Maritime deaf community who, according to his stories, had misguided ideas - and took action accordingly — about the importance of sign languages in the Maritimes (MSL and ASL) and the role of sign language instruction in developing stronger communities and services, as described below.

In the next section, I revisit the stories from Chapter Eight, which focused on the teacher-participants' pathways forward.

\section{Summary of Chapter Eight}

This chapter was guided by layers of chronology, as the teachers' stories within simultaneously pointed backwards at their prior experiences with teaching and learning and projected forward their visions for the future. The teachers' stories in Chapter Eight 
clustered around three topics: teaching for the self, teaching for the students, and teaching for a better world, as described below.

- ASL teaching was a promising life direction for Nick and Nathalie, both of whom had recently experienced major upheaval in their personal lives and found a safe haven in ASL teaching. All of the teacher-participants described their dedication to ASL teaching as a career.

- ASL teachers educated students about audism and hearing privilege by reinforcing that ASL had a long history of being suppressed as well as cherished and had been passed along through generations of deaf people who themselves had long been targets of discrimination as well as drivers of social change.

- ASL teachers felt able to provoke meaningful social change as they had or aimed to have a direct impact on the next generations of hearing signers who went on to become interpreters and allies of the deaf community, or at least left their ASL classroom with a new respect for sign languages and deaf people.

Having provided a high-level summary of the stories from Chapters Six to Eight, the next section draw together the threads that emerged from these stories to lend coherence and closure to the findings of this dissertation study.

\section{Weaving the Stories into the Tapestry}

Hollingsworth, Dybdahl, and Minarik (1993) described their work as informed by “an intersecting tapestry of theoretical perspectives" (p. 8, italics added), including theories of self/other relationships, the social construction of knowledge, and feminist epistemologies. The study itself was less a report of teacher knowledge and more of an unfolding or interweaving of stories of experience from two teachers (i.e., Dybdahl and 
Minarik) drawn from extensive conversations with the researcher (i.e., Hollingsworth). In addition to borrowing their metaphor of a theoretical tapestry to frame my own influences (Chapter Three), I was also inspired by their study, in particular the sensitivity and attention they paid to the teachers' knowing through practice, reflection, and conversation, and the relationships between all three. In my own dissertation, I, too, aimed to bring value to the many ways that ASL teachers know, both formally through external (to the teacher) standardized knowledge acquisition outlets (e.g., teacher training, published literature, textbooks) and, more importantly to me, in personalprofessional practice. Like Hollingsworth and her co-authors (1993) and the several other scholars whose work informed this thesis (Clandinin and Connelly, 1996, 2000; Connelly \& Clandinin, 1988; Elbaz, 1995; Elbaz-Luwisch, 2005; Schön, 1983; see Chapter Three), I approached the stories shared in this study with a dialectic understanding of the relationship between theory and practice. I held that experience, or practice, generates theories that feed back into and drive practice. Theories may be derived from outside, such as theories of second language pedagogy from empirical literature, but these will be filtered through the teacher' own understandings and personal theories of teaching and learning.

Looking across the stories in Chapters Six to Eight, summarized above, there were many threads that ran through them that reflected the dialectical relationship between ASL teacher-participants' stories and the theoretical and empirical literature that guided this study (described in Chapters Two and Three). The relationship between the stories and the literature was depicted in Figure 3. An expansion of Figure 2 (Chapter Three), Figure 3 summarized the interrelationships between the study's teacher- 
participants' stories, threads, and aims of this study, with the addition of specific threads and contributions. I describe each component of Figure 3 in the paragraphs that follow.

The vertical axis was a reminder that I began this research with the goal of highlighting the ASL teacher-participants' storied pathways; the stories were the point of departure. From those pathways, I drew out narrative threads that wove the pathways into conversation with the study's guiding theoretical and empirical framework. Finally, the pathways and the threads had several potential academic and practical contributions for the ASL teacher-participants, their peers, and the profession more broadly.

The arrow along the horizontal axis was a reminder of the temporal structure of this study, where teacher-participants' stories related to different points along their past, present, and future pathways, and these occurred along a continuum.

Moving upwards from the teacher-participants' stories and feeding back into them were the themes of the study (knowing-in-action, narrative as social action, the sociohistorical context of ASL, and common ASL teaching practices; described in Chapter Three). A narrative analysis of the teachers' stories (as described in Chapters Three and Four) across the three dimensions of narrative - sociality (intra- and interpersonal interactions), temporality (time), and space (and place) — provided context that helped to illuminate the stories' threads in relation to those themes. The themes themselves helped to draw out the meaningfulness of the teachers' stories in theoretical and empirical terms. 


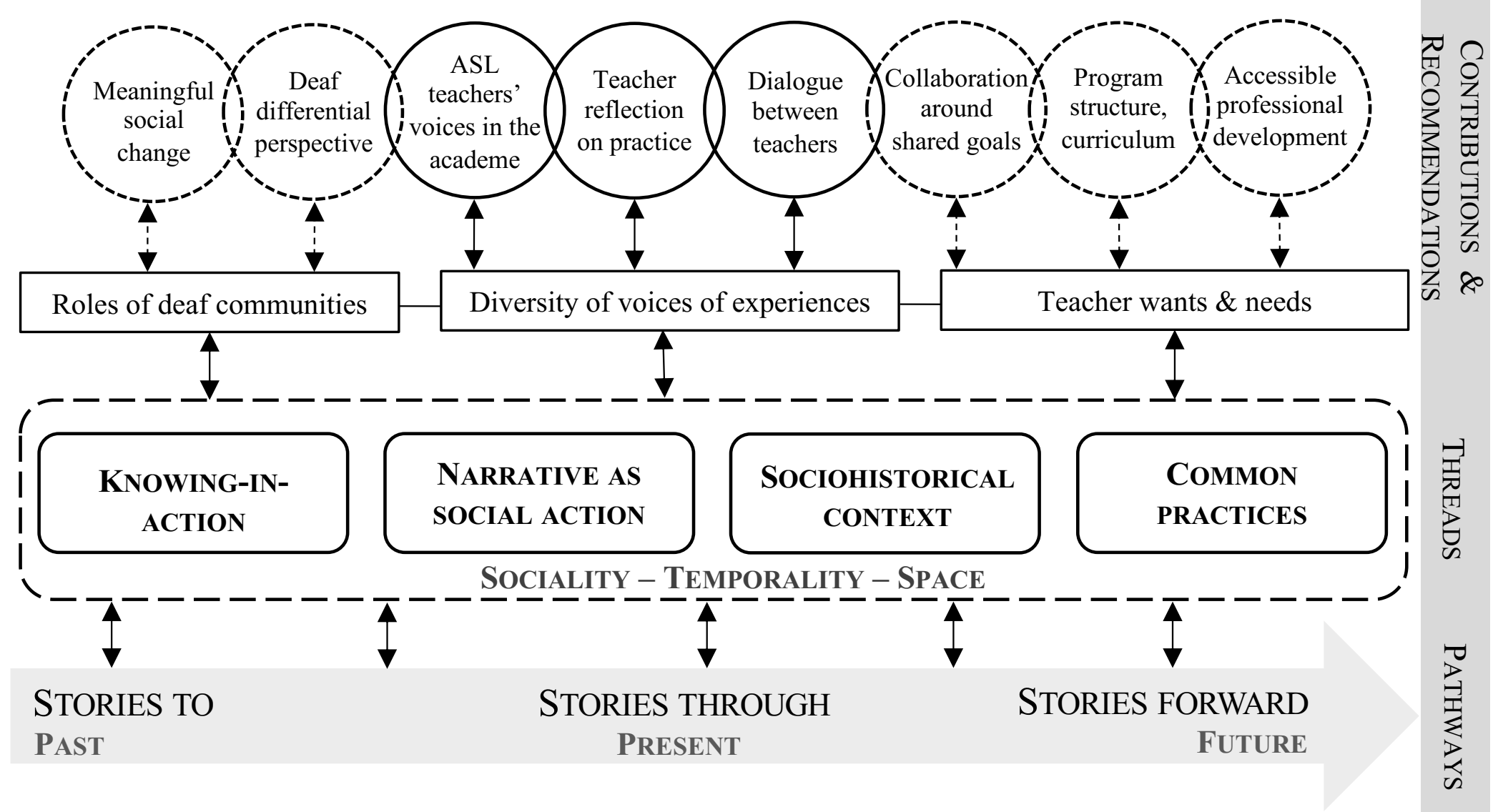

Figure 3. Relationship between stories, threads, and contributions and recommendations. 
At the top of the figure were several of the contributions this work may have made, directly (circles with solid lines) or potentially (circles with dotted lines). I organized the contributions into three interconnected groupings: contributions related to (1) the roles of deaf communities in ASL teaching; (2) the diversity of ASL teachers' voices and experiences; (3) teachers' professional wants and needs, currently and moving forward.

As a reminder that theory and practice are in constant dialogue, and that the contributions of this study were drawn from stories that showed but a snapshot in time (Chapter Four), all of the arrows in Figure 3 were bidirectional.

The remainder of this section attends to each of the study's overarching themes in turn. Each section begins with a framing quote that captures the essence of the theoretical or empirical stance that binds the section. I begin with a discussion of how this study's ASL teacher-participants' stories reflected knowing-in-action (Elbaz, 1981; Lave, 1993; Schön, 1983).

\section{Knowing-in-action}

Traditionally, learning researchers have studied learning as if it were a process contained in the mind of the learner and have ignored the lived-in world... A more promising alternative lies in treating relations among person, activity, and situation, as they are given in social practice, itself viewed as a single encompassing theoretical entity. (Lave, 1993, p. 6-7)

As discussed in the opening of this section and reiterated in the above quote by Lave, teachers develop their knowledge, or the more active knowing as per Hollingsworth et al. (1993), through practice. This stance, which underpinned the present dissertation, evoked the work of several generations of scholars whose 
work was discussed in Chapter Three-for example, Schön (1983) who was inspired by Dewey (1913), Clandinin and Connelly (1996, 2000; Connelly \& Clandinin 1988) who were inspired by Elbaz (1981; Elbaz-Luwisch, 2005) and Lampert (1985), and Craig $(1999,2007)$ who built on all of the above. All of these scholars held that practice itself is theory-driven, and reflection on practice can provide teachers with opportunities to explore, challenge, and learn from their developing theories of practice as they move through their professional landscapes.

In this study, the ASL teacher-participants' stories showed tremendous reflection and growth through their practice. For instance, their stories of the classroom - their use and development of curricular materials, their interactions with students, etc.- - illustrated different ways that their teaching has been informed by what has worked or not in the past and what they hope to accomplish in the future. These forward-looking stories in particular were often grounded in personal theories of teaching and learning derived from past experiences as a teacher and as a student and values and goals about the role of ASL in the future.

The stories also showed growth in their professional identities, where the teachers' stories described their participation and learning through training, reflections on their practices that led to updates in their everyday practices. Across many of their stories, they described their efforts at developing themselves as knowledgeable and confident professionals.

Related to this, the stories that teacher-participants shared in this study showed how their practice and reflections on practice helped them to develop 
personal theories of the self in addition to theories of teaching and learning. For many of the teachers, learning to teach ASL was a source of empowerment, of taking ownership of their language and culture, and reclaiming the relationships of mutual respect with the hearing students in their classes.

Ultimately, teachers' stories of their teaching practices stayed close to their stories of their ASL and deaf community practices. As they moved through their work as teachers, many of them kept their cultural knowing close by or, more often, interwoven with their professional practice. This was one of the main threads of this study - that ASL teaching cannot be considered separately from the cultural and sociohistorical context of ASL. I revisit this idea later in this chapter. Next, I focus on how teachers' stories reflected theories of narrative as enabled and constrained social action.

\section{Narrative as social action}

We achieve our personal identities and self-concept through the use of the narrative configuration, and make our existence into a whole by understanding it as an expression of a single unfolding and developing story. (Polkinghorne, 1988, p. 150)

The above quote from Polkinghorne points to the undercurrent of this entire dissertation: people tell stories to make sense of themselves, their worlds, and the relationships between both. In this study, the ASL teacher-participants' stories were in conversation with many other kinds of stories that both enabled and constrained them.

The clearest instances of the social nature of stories were the narratives in Chapter Seven that related to ways of being a "good" and "imperfect" teacher. These stories were 
doing things like asserting "good" and "bad" teaching approaches, professional practices and values, ways of interacting with the deaf community, and so on. These stances lived in the stories as much as they were enacted in practice (although without observing teaching in practice, I could not begin to say where they lived most fully). Likewise, these stories were in conversation with the sociohistorical context of ASL (Chapter Two) and common ASL teaching practices (Chapter Three), as described in the upcoming sections. Because I was familiar with these contexts and practices, I was able to identify them in the teachers' stories.

One example was Nathalie and Nick's stories of allowing voicing in their teaching - Nathalie in her private conversations with students and Nick among students in the classroom. The stories they told (see Chapter Seven) and especially how they told them said much about the grand narrative (and common practice) of ASL teaching: the no-voice policy. Both Nathalie and Nick demonstrated in their storytelling that they were aware that voicing was taboo in ASL classes by framing their decisions to allow voice as a rationalization, a conscious resistance to one aspect of the sacred story that drove ASL teaching.

These and other stories told not only of how teachers saw themselves and how they strove to be, but also how other teachers were and how they should strive to be. For example, Erica and Donna told stories of being unable to engage new teachers to take on a professional sense of responsibility and work ethic that met their standards. These kinds of stories would be enlightening in future efforts to develop professional development programs and perhaps even structured mentorship opportunities where teachers would be in a position to share these narratives of professionalism with each other. Likewise, such 
stories could give insight into the types of curricular reform that might effectively align with teachers' stories.

Another example was the many stories of deaf empowerment through learning to teach. These stories-for example, Ken learning about teaching ASL, learning more about English, and learning to relate to his hearing learners through mentorship and personal reflection on practice- spoke back to and rejected the narratives of DEAF CAN'T that had permeated many aspects of their lives. Through ASL teaching, they were able to tell their story as one of empowerment through professionalism.

As a final point, writing this dissertation was in itself an interpretive and social act. My intentions, which I describe later in this chapter, included bringing ASL teachers' voices into academic conversations. In doing so, I positioned teachers as authorities on their own practice, which, as explained above, is intimately tied to their own lives experiences. Their stories, frozen as they are in this static document, have already been engaged in scholarly discussions throughout this dissertation and, upon publication of this dissertation, will soon be available for further scholarly engagement.

\section{Sociohistorical context of ASL}

One cannot consider linguistic aspects of ASL teaching without taking into account the unique history of ASL instruction over the years. The teaching of ASL is superposed on the socio-cultural history of the Deaf community - a history that has been influenced greatly by interactions between Deaf and non-Deaf (i.e., hearing) people. (Quinto-Pozos, 2011, p. 138)

As mentioned above, the ASL teacher-participants' stories of practice were often tightly bound with their awareness of the sociohistorical context of 
ASL and deaf people. I provided an overview of this context in Chapter Two as a way of helping the reader to grasp the truth of what Quinto-Pozos stated above in the framing quote for this section.

The sociohistorical context that was most relevant to this study included the ongoing suppression of sign languages (e.g., Erica's explanation of the status of ASL in her Prairies province), ongoing misconceptions and discrimination against deaf people (e.g., Ken's experiences of audism), and, to some extent, the history of horizontal violence within deaf communities (e.g., Michelle and Nathalie's experiences being oral deaf/hard-of-hearing). The latter case was especially visible in Nathalie's stories of struggling to build up her confidence and comfort in the profession, of staking her claim as a professional on the field, while facing the grand narrative of the deaf ASL teacher, which lived in real life in the negative behaviours of some people in the deaf community.

Lastly, the sociohistorical context of ASL was perhaps most present in teachers' stories of their visions for the future. Ken, Donna, and Erica all shared powerful stories of using their teaching as a way to reframe negative grand narratives about deaf people (e.g., disability model) that were embedded in hearing learners' minds. Like David, their stories were about ASL teaching as a campaign of awareness for positive social change.

\section{Common practices}

This curriculum... will continue to demonstrate that ASL is the subtle, elegant, powerful language or a rich, complex culture. (Smith, 1988, p. 181) 
Because this study's methodology did not include ethnographic observation, there was little to be said about teachers' actual practices or the impact thereof on the teachers themselves or on their peers, students, or communities. Still, looking to Smith's quote above, the stories of practice of the ASL teachers in this study reflected many of the common practices that were described in previous literature (Chapter Three). As mentioned previously, the no-voice rule was common in classrooms. Likewise, Signing Naturally featured prominently in several teachers' practice, albeit with many adaptations.

The most powerful observation about teachers' practices has already been discussed above — namely, that teachers' classroom practices are drawn from their own past and ongoing experiences and the grand narratives that drive the profession, which themselves are intertwined with the sociohistorical context of ASL. Although Figure 3 showed a two-dimensional and fairly compartmentalized relationship between these themes and the present discussion organized them linearly, the reality is that one is not the starting point for the other; rather, they are a dynamic layering of lenses that, taken together, show the richness of teachers' stories of practice.

In closing, having summarized above the stories and discussed the threads that emerged from Chapters Six to Eight in relation to the theoretical and empirical literature that informed this research, I now turn to a discussion of how the findings of this study began to address the intended contributions outlined in Chapter One.

\section{Potential Contributions and Recommendations}

In Chapter One I proposed six intended contributions of this study. In this section, I discuss how this study may have responded to each contribution. When applicable, I 
offer recommendations that may help to guide ASL teachers in their ongoing practice and to develop the profession more generally.

- Contribution 1: Bring ASL teachers' voices into the academe. As described in Chapters Two and Three, applied sign linguistics, and in particular the study of ASL teaching for hearing adult learners, is a relatively recently established field of academic study and does not yet have a robust theoretical or empirical foundation, although it is certainly growing. Applied sign linguists can certainly draw from applied linguistics to some extent, but given the distinct nature of sign language discourse, the sociohistorical context of sign languages (see Chapter Two), and the common practices of ASL teaching as a profession (see Chapter Three), many traditional theories - i.e., those grounded in spoken, often majority, language-centric contexts - do not fully apply. Thus, with this study, I aimed to contribute to applied sign linguistics scholarship with the present empirical research to build up discussions in the field, and to applied linguistics by offering empirical evidence of several practicing ASL teachers' sign language and deafinformed perspective on language teaching and learning and language teachers' professional practice.

- Contribution 2: Increase dialogue between ASL teachers in Canada. As Doré (2015) also found, there was significant diversity amongst the ASL teacherparticipants in this study in terms of their language and educational backgrounds, the types of professional development they engaged in, and the successes and struggles they experienced in their collegial relationships. Most of the teacherparticipants also described having few occasions to collaborate with fellow ASL 
teachers and a desire for more. In response, the portraits and stories presented in this dissertation will hopefully provide future ASL teacher-readers with a window into the experiences of their peers, and perhaps help them to identify possible points of commonality or difference and inspiration or self-reflection. The process of conducting members' checks (described in Chapter Four) already provided insight into the types of questions teachers have about each other-e.g., What materials do they use? Do they talk in class? Are they deaf? Which workshops do they go to? How did my background compare to others'?

A more concrete recommendation to help ASL teachers develop more and stronger professional relationships moving forward is to promote gatherings of teachers, whether at the local level (e.g., within a program) or otherwise (e.g., SLIC, Canada-wide), around "wicked problems" in the profession. Fox (2009) found Brown's (1995) delphi technique, a consensus-building technique, to be useful in the context of a university language program seeking to reform its curriculum. The delphi technique provided the divided faculty in Fox' study a "neutral site" (p. 34) for decision-making. Such an approach might be valuable in ASL teaching to help teachers come together to define common teaching-focused aims "from the ground up". ASL program administrators might play a role in facilitating this process for program-specific decisions, as discussed further below.

\section{- Contribution 3: Provide ASL program administrators with insights} regarding ASL programs and teachers. The ASL teacher stories and literature (Chapters Two and Three) presented in this dissertation may also be enlightening 
for ASL program administrators who are unfamiliar with the profession, how ASL teachers and students may have unique needs and issues distinct from spoken language contexts. For instance, the central role of the deaf community in decision-making around ASL teaching would be valuable to reinforce. As I reflect in the upcoming Chapter Ten, tensions arise when ASL teachers do not or do not appear to be in close consultation with members of the language and cultural community. Administrators might find it useful to know some of the common teaching practices and professional development opportunities that may be common or available to ongoing or prospective ASL teachers in their program.

In practical terms, and extending my point from the previous section, ASL program administrators and supervisors are well-positioned to address recurrent issues that emerged in the ASL teachers' stories in this dissertation: collegiality and collaboration are not uniformly engrained in ASL teachers' professional practice. For instance, there was no agreed upon structure and content in the ASL program where Nathalie worked, which put the bulk of the decision-making in her hands. She was aware of the gaps in the coordination of her program and ready to take on the challenge being a former student in the program, but incoming teachers to the program may have faced more significant issues in building the insider knowledge and alignment that came easily to Nathalie. Using a selection of the Signing Naturally series, for example, might provide a baseline level of standardization that teachers can build on. As one of the ASL program administrator-participants in this study said, he considers himself accountable to 
students in the program and has been working on engaging his department's ASL program to engage in program review as an exercise in introspection.

- Contribution 4: Provide ASL teacher-participants the opportunity to reflect on their practice. Just as the members' checks invited the ASL teacherparticipants to consider other teachers' practice, their initial participation in our multi-part interviews was itself a form of potentially valuable reflection. As described in Chapter Three, according to Schön (1983), reflection on practice is itself a rich source of learning. The stories in Chapter Eight were especially enlightening about teachers' interpretations of their past experiences and what they drew from it that might inform their future practice.

Likewise, reflecting on their extensive profession pathways might have provided ASL teacher-participants with an opportunity to relive moments of success, joy, and growth, which might feed back into their practice. For example, Ken telling me stories about his early days of teaching alongside stories about the extensive trainings he has completed and led may have been a confidence boost as he relived the empowerment of those experiences all over again.

\section{- Contribution 5: Highlight ways that ASL teachers can effect social change} through their work. Much like the point above, in this study, the ASL teacherparticipants had the opportunity to reflect and perhaps draw inspiration from articulating their dreams and aims for their work. For example, Erica, Donna, Ken, and David all had strong motivations for their teaching that related to creating a better world for deaf people. 
- Contribution 6: Provide a foundation for planning for future professional development. As mentioned in the sections above, this research has provided ample insight into the lived experiences of several ASL teachers that may, in turn, give some direction to potentially valuable professional development opportunities. For example, above I suggested that the delphi technique (Brown, 1985, as cited in Fox, 2009) might be worthwhile as an approach to gather teachers to exchange and develop ideas about teaching and learning ASL to hearing adults. To align with a deaf cultural perspective, future professional development should be organized by or closely with deaf cultural organizations. Like McKee and Woodward (2014) found, sign language teaching communities can be good avenues for developing stronger deaf communities. A deaf differential approach to developing the profession might make it a more viable option for deaf youth seeking to become teachers.

In closing, in this chapter I summarized the ASL teacher-participants' stories from Chapters Six to Eight and the narrative threads that emerged from them, and I reflected on the possible contributions of the stories and threads in theoretical, empirical, and practical terms. In the next chapter, I take a step away from the teacher-participants' stories to reflect on my own stories of experiences throughout the years of engaging with this research. 


\section{Chapter Ten: Reflections on My Research Journey}

In this chapter I step away from the findings of the study to consider my own experiences as I undertook this doctoral work. Throughout this dissertation, I have attempted to do justice to the teacher-participants who shared their stories so openly and thoughtfully. While I feel that I accomplished that goal, the process of conducting this research was not without challenges and doubts, principally around my role and legitimacy as a researcher in this area where I was largely an outsider.

To process my relationship to this research, I wrote over 1,000 journal entries from 2014 to 2020, chronicling the mundane to the intensely exciting moments of my dissertation journey. Many of these entries were analytic in nature and used to support my data analysis. Many more were accounts of and reflections on the day-to-day of getting lost and found in this work. I revisited these entries toward the end of this dissertation process as a form of catharsis. In the sections below, I explain two events that struck a particular chord and were illustrative of some of the "stickiness" of my experiences throughout this dissertation. Most of all, these events show how complex my positioning as a researcher was from the beginning to the end of this work.

Student researchers may find these reflections relatable, as I candidly discuss the inner workings of graduate studies that often lose their place in the final dissertation product. A methodology-minded reader may appreciate these reflections as an effort to give insight into the lens that tinted my interpretations in the previous chapters. Sign language scholars may find these reflections useful, as I ponder relevant but underexplored topics in the field, such as the personal impact of confronting hearing privilege and conflicting stances in the deaf and sign language communities. Finally, 
these reflections have been immensely helpful for me because they gave me a space to appreciate the evolution and intricacies of my learning and the diversity of experiences I have confronted throughout this dissertation, all of which have affected my research decisions and contributed to a growing skill set.

Overall, it has been a long journey to the point of assembling this dissertation and eventful enough for me to consider it an adventure. Having previously completed a Master's about ASL teachers' curriculum experiences, I was aware that there would be some hurdles to completing a larger-scale project in this area of study. I tiptoed into this Ph.D. with ample imposter syndrome—should I be doing this research? Who will benefit from this research, really? Part of the hesitation was because I began learning ASL in my early twenties and no matter how readily—and proudly—I absorb new dialectical features when meeting a new signer, I hold my elbows too high, my finger spelling is stuttered, my eye line darts off too often. Moreover, I am a hearing person, which can carry a social currency and mobility not afforded to some of the deaf people who are involved with and may be impacted by this research. I was conscious about my work being self-serving and not useful to the ASL teaching community, as intended.

There were also several moments of tension early on as I found points of friction between academic disciplinary (applied linguistics) and the broader context of ASL teaching. In brief, early on, several leaders in the deaf community were concerned about my "interference" with ASL-related matters, a well-founded concern given my relatively outsider status in even my local sign language community. Yet when I began interviewing individual teachers for this dissertation, I found little reason to stay on figurative eggshells. The teachers I spoke with were welcoming, energetic intellectuals 
who were excited to speak with a fellow educator, despite (or perhaps because of) my "hearingness" (Sutton-Spence \& West, 2011) and spotty signing. We connected as teachers, bonding over teaching successes and failures, over caring for our students, over 'bigger picture' questions about the impact of our work, about the future of our respective language communities. This in itself was a valuable learning experience for me.

The first story I share below is about an event that I was not directly implicated in but was ultimately affected by in several ways. In a nutshell, the event involved the circulation of an online petition (appended in full in Appendix L) protesting the hiring of a hearing full-time ASL instructor at Carleton University. The petition was penned by several prominent deaf leaders, including representatives from CCSD and the SLIC who cut ties with me as a result of my being a student at Carleton University. Before I go further into the story about the petition, I should flag for the reader that I have opted not to anonymize the names of the organizations (e.g., CCSD, SLIC) or the institution (i.e., Carleton University) involved in this event, although I did remove specific authors' names for privacy. The petition was a public and widely circulated web document, and it continued to be publicly available at the time of this dissertation's publication. Being an online petition, the authors arguably wrote the document for public consumption and with the specific aim of maximizing the petition's visibility, with their names and respective affiliations likewise prominently displayed. Thus, I felt that it was not against the authors' original intentions to include the document in full (minus the authors' names) here. For me to anonymize the organizations and institution in the petition herein would be to minimize the impact and relevance of the petition itself, for it contains valid and valuable arguments from critical people in the deaf community. Although I question 
some of its points, by including the petition here in its full form I can potentially increase its reach, or at least the reach of its key arguments. Additionally, the collapse of my relationship with the SLIC was a direct result of my affiliation with Carleton University; to anonymize or otherwise blur identifying features of the petition would have failed to capture the impact of the petition on my study. As a final note, I want to stress that the petition was circulated after most of my data collection had already taken place and that the ASL teacher-participants in this study were from across Canada, coast to coast, and may or may not have been impacted by it.

Having explained my rationale for presenting the full details of the petition, I now describe the event in more detail and its impact on my Ph.D. work.

\section{The Petition}

After most of my data collection had wrapped up, a petition began circulating in the Internet about the Carleton University Modern Languages department's recently hired a full-time hearing ASL instructor. Within two days, the petition had collected over 500 signatures and 150 comments that echoed the petition's anger and disappointment about the new teacher (Appendix L). The petition was led by representatives of the CCSD and the SLIC, as well as nine other prominent figures in the Canadian scholarly and community ASL and deaf cultural landscape.

The arguments put forth were compelling. The authors referenced Robinson and Henner's (2018) stance on “cripping” (i.e., bringing a disability lens based in discourses of empowerment) the university through ASL instruction, which highlights the nonreciprocal, "exploitative relationship" (to cite the petition) between the university and local deaf communities. The petition argued that Carleton profited financially from ASL 
courses through ample enrolments - the ASL program comprised over 1,000 students every year-with little connection to the wider Ottawa deaf community, such as through advocacy work about such pressing issues as improving deaf education and recognition of sign language rights.

The petition likewise expressed disappointment in the program's internal structure. For instance, the hiring committee did not require applicants to hold a minimum ASL proficiency score (e.g., ASLPI) and the program "rejects the use of ASL curricula that have been developed by and with deaf ASL communities and that require training by deaf community organizations". The contention was that Carleton's in-house qualification standards and curriculum did not support collective deaf-led efforts such as those described in Chapters Two and Three and echoed Donna's stance on supporting deaf-authored teaching material (e.g., Signing Naturally).

Finally, the petition said that ASL as a second language (L2) and a second (signed) modality (M2) required significant investment and that Carleton's ASL students were ill-prepared to become interpreters who will “directly impact deaf people's access to employment, healthcare and other public services". McDermid (2012) expressed similar concern that hearing learners never achieve full mastery. This finding is troubling for the many deaf adults who rely on interpretation services and deaf children for whom their interpreters are primary language models.

On the one hand, these points were well-founded, as deaf paternalism and hearing hegemony are nothing new, whether in the form of hearing-based or audiocentric discrimination (i.e., audism; Eckert \& Rowley, 2013; Humphries, 1977), non-deaf leadership of organizations and institutions for deaf people (audistocracy; Beatty, 2015), 
and/or the systemic privileging of sound (phonocentrism; Bauman, 2008). It was unsurprising that deaf-led initiatives such as the ASLPI and deaf-authored materials were promoted by the authors of the petition: they can accomplish the dual function of providing instruction that aligns with deaf paradigms and empowering the deaf community (DEAF CAN) through self-definition. Plus, the success of any ASL foreign language program may be salt on a long-standing wound (also described in Chapter Two):

... [A]lthough this is rarely discussed openly - in private, many will reluctantly acknowledge that while deaf children's chances of acquiring fluent signing skills are often reduced by the absence of appropriate language models in their upbringing, and hearing learners of signed languages can receive deliberate, wellcrafted language teaching, it becomes increasingly likely in the present era that some hearing people may start to become more capable signers than some Deaf community members. (Turner, 2009, p. 250)

In other words, the petition stated the blunt truth: at the same time as ASL as a foreign language programs are booming in secondary and post-secondary institutions across North America, deaf people are fighting for access, recognition, and rights to sign language and deaf culture, especially for deaf children.

On the other hand, I felt several points deserved contextualization in the context of this dissertation. While it was undoubtedly concerning that weak signers are entering the community as poor-performing interpreters, there is a 9:1 ratio of beginner to advanced learners (Looney \& Lusin, 2018), which means that few learners will make it into, let alone complete, an interpretation program. Instead of being burdens on the 
language community, even barely-proficient ASL students may represent a significant opportunity to shift broader social attitudes about sign languages and deaf people (as per long-standing prior research, e.g., Kannapell, 1989 and the literature in Chapter Three).

Finally, the petition conspicuously omitted details about the candidate besides their hearing status but alluded to their hearingness being responsible for all of the various problems with Carleton's ASL program. It was unclear whether the Carleton teacher was an L1 or L2 signer, for example. I knew from my earlier study (Doré, 2015) that there were many hearing and hard-of-hearing ASL teachers across Canada, including several ASL L1 coda teachers, and many more deaf teachers who had learned ASL as L2, including several teachers in this study. Not only is the case of the native signer highly nuanced and concern over deaf ASL teachers' qualifications is complex (see Chapters Two and Three), this stance ran contrary to decades' worth of research that has considered non-native teachers as valuable practitioners (e.g., the scholarship of Davies (1991), Cook (1999), and Paikeday (1985), for example). The petition's contention that teachers used spoken English in the classroom to the detriment of learning also ran contrary to recent research such as about pedagogical translanguaging, which encourages language learners to access their entire linguistic repertoire when learning an additional language (e.g., Cenoz \& Gorter, 2017).

All in all, I read the petition with no small amount of conflicted feelings. It called attention to issues that are pervasive in ASL teaching across North America and hopefully opened a much-needed dialogue within programs regarding priorities for teachers, students, and the wider community. It also echoed several of this study's 
teacher-participants' stances about, for example, the role of the deaf community in ASL teaching, which was enlightening for me.

Yet, the petition also based many of its points on arguments that clashed with the state-of-the art in language instruction research. In a way, I built my academic argument and the present dissertation around this dissonance. I have found that ASL as a foreign language for hearing adult learners is fraught because of fundamental paradigmatic differences about who should teach, with what/whose materials and methods, to which learners, what and how much to teach, to what end, and so forth. Well-established norms and baseline criteria in (spoken) language education do not always apply-e.g., nonnative language users are embraced as teachers, teachers are "qualified" through linguistic and pedagogical training, curricular materials should be research-based. Instead, competing cultural, linguistic, and political paradigms of the deaf and sign language community sometimes take precedence. Throughout this dissertation, I hope to have illustrated that applied linguistics literature does not yet adequately account for the particularities of the ASL context, and to do so it must consider openly and entirely the experiences of diverse of teachers, such as those who shared their stories herein.

The next story, which I share below, describes a brief but thought-provoking encounter I had with a fellow hearing person who also worked closely with deaf people and community leaders and faced her own challenges along the way.

\section{Audistocracy}

In the last year of writing this dissertation, my supervisor nudged me to meet with a local woman whom I will call June (not her real name). June, only a handful of years earlier, had established a deaf adult support program in Ottawa. As a non-signing hearing 
person making decisions that affect deaf people, June had experienced a significant amount of pushback and skepticism from many deaf community members who resented her attempts to host the program through a hearing organization in what appeared to be a paternalistic takeover or, at the very least, an unwelcome intrusion by an "outsider". The program was a success eventually, but June reflected that it had taken several years and significant advocacy from supportive community members to build meaningful collaborations and trust with deaf leaders, some of whom she described as initially "ferocious", despite their shared vision.

In brief, June's good intentions proved threatening. Indeed, deaf people have a long history of resistance to what has been termed audistocracy. Audistocracy has been defined as "institutions, organizations, and programs ineptly run by non-deaf people for the benefit of Deaf people and Deaf children without, or with minimal, involvement by the Deaf community" (Simmons \& Beatty, 2015). The term was adapted from kakistocracy, an early $19^{\text {th }}$ century term for a government run by "the least suitable, able, or experienced people" (“Kakistocracy”, n.d.).

One of the most prominent examples of deaf people overturning an audistocratic institution is the Gallaudet University's Deaf President Now (DPN) movement in the late 1980s that resulted in the appointment of the institution's first deaf president. Jankowski (1990, as cited in Haller 1992) somewhat controversially compared DPN to a plantation uprising where the slaves are eventually liberated. This is not a far cry from deaf studies scholars who compare deaf oppression by hearing society to colonialism and Indigenoussettler relations (Ladd \& Lane, 2013). While this might seem like hyperbole, there are clear grounds for resistance to hearing hegemony. 
The meeting with June resonated with me, as throughout this dissertation research I also struggled with establishing common ground and mutual understanding with prominent figures in the deaf community. While I was warmly welcomed by ASL teachers - into their worlds, their stories, and even their homes - the reception was not as warm from the several community leaders with whom I attempted to engage to accomplish the first version of this study. The details of our relations are unnecessary to recount here, only that the positive relationships I had aimed to cultivate with deaf community leaders were unsuccessful. On the one hand, I am still disappointed that my attempts at collaboration were rejected. On the other hand, I understand where the resistance came from, as I, too, doubted my own intentions, place, and potential for contributions. At the very least, I learned from the experience to expect both the unexpected and the expected.

Although this dissertation has come to a close, I continue to live in the above and more debates about my research. Through it all, I remain optimistic that post-secondary sign language instruction is more than the manifestation and perpetuation of hearing dominance; it is also fertile grounds for the cultivation of positive hearing-deaf relationships. In short, I believe in the transformative potential of language learning (Norton \& Toohey, 2004), particularly of sign language learning, where there exists the potential to increase the hearing population of hearing people who are better-equipped to build informed relationships with deaf people (Peterson, 1999; Sutherland, 2008). Growing the language and extending its use is one way of reaching this ideal, although it has been and will continue to be a "sticky" journey for many years to come. 
In the following chapter, Chapter Eleven, I conclude this dissertation by considering some of the limitations of the present study and possibilities for future research directions. 


\section{Chapter Eleven: Conclusion}

Having summarized the main findings and contributions of this dissertation in Chapter Nine and reflected on my own journey through this research in Chapter Ten, this concluding chapter identifies limitations of the study and propose directions for future research.

\section{Limitations and Directions for Future Research}

Although this study of ASL teachers' pathways to, through, and forward in their profession led to many rich and insightful stories, a limitation was that my findings were based solely on teachers' own accounts through conversations that extended over several weeks to months. I did not engage in any in-person observations (e.g., in class, at meetings), nor was my relationship with the teachers extensive in research terms. In narrative inquiry, so much depends on the nature of the narrator-narrative style (e.g., information-sharing vs. storytelling), positioning in relation to the researcher (e.g., insider-outsider), biases, etc. Observation can provide an additional layer of insight. As Elbaz-Luwisch (2007) encouraged observation as a way to "understand[] thought and action not as two clearly distinct phenomena or events that make up the work of teaching but as interconnected and complementary sides to the unified experience of the teacher" (p. 360). In this study, then, my interpretations were based on only a small window into teacher-participants' worlds, and thus a starting point for additional research. A strong direction would be to continue this work with the addition of ethnographic observation, ideally over an extended period of time, to better understand their stories in action in the particular contexts of their work. Alternatively, a targeted case study approach might be 
beneficial, particularly to inform ASL teacher training curricula and future programs of study.

It would be valuable to address in more depth the ways that ASL teachers connect with one another, particularly across time and space through things like shared curricular materials and theoretical ideas about the nature of teaching a minority language. This type of study would perhaps provide more and more practical recommendations for practice (professional development activities, mentorship programs, etc.). A potential guiding theoretical construct for this work could be Parry's (2008) construct of intimacy. Parry (2008) reflected on the potential for physical artifacts - in her case, deceased author Iris Murdoch's brain— to bind people and create spaces of intimacy beyond time, space, interaction, and even language. Alternatively, Appadurai's (1991) theory of ethnoscapes could help to re-imagine ASL teaching as a "non-localized" -scape:

The landscapes of group identity — the ethnoscapes — around the world are no longer familiar anthropological objects, insofar as groups are no longer tightly territorialized, spatially bounded, historically unselfconscious, or culturally homogeneous. (p. 48)

Although not explored in this dissertation, either direction could help further develop a finding from Doré (2015), namely that ASL teachers overwhelmingly felt part of a teaching community and yet actual interaction with other teachers was rare. As Thoryk (2010; as well as Rosen, 2010, and Swaney \& Smith, 2017) noted, there is a tendency for ASL teachers to favour well-known deaf-authored materials regardless of their being upto-date, empirically derived, theoretically based, and so on. It might be, then, that the development of intimacy or non-localized cultural-professional "-scapes" through 
curricular materials accounts for ways that teachers feel connected. Future studies could explore this idea further, perhaps through the lens of curricular innovation (e.g., how curricular materials gain popularity) or teacher identity development (e.g., how identity development relates to using the "right" teaching materials).

Related to this, it would be helpful to teachers and professional organizations if future research addressed issues around the reportedly disjointed nature of informal professional development activities (e.g., informal mentorship, on the job training), especially in light of the pervasive trend for professions to standardize, formalize, and credentialize. If ASL follows that direction, who would standardize and disseminate materials and training, especially given ASL teaching organizations like the SLIC and deaf cultural organizations are volunteer-led? Likewise, what is a reasonable scope of training given ASL teaching is largely part-time work? Atherton and Barnes (2012), Gordon and Harvey (2009), and Goertzen (2018) all found that the result of inflexible or unattainable qualification requirements is often that hearing teachers are hired, which is not considered an acceptable practice.

A further examination of the theories and ideologies that underpin formal ASL teacher training programs would be informative, especially if it would help to articulate a deaf pedagogy as distinct from spoken language pedagogies. This research is already underway to some extent; for example, Hermann-Shores (2017) proposed that $21^{\text {st }}$ century ASL instruction should shift from a teaching curriculum that focuses on instruction to a focus on learners as members of a shared community of practice. Teaching practices could include collaboration between students and other learner- 
centered practices. It would be worth delving deeper into how ideas such as these are filtered into classrooms through teacher training programs or other channels.

A final and highly worthwhile direction to pursue in future work is to consider in qualitative and quantitative terms the impact that ASL teachers have on the wider hearing community through their work, whether intended, perceived, or real. Elbaz-Luwisch's (2005) work about attentiveness to teacher's stories and her application of Bakhtin's theory of polyphony would be a helpful structuring frame, as the multiplicity of teachers' inner voices (as teacher, as deaf person, as cultural minority, etc.) could be brought forward. Or, looking outwardly to Canada more generally, a country defined by its broad cultural and ethnic landscape, it could be meaningful to explore how ASL teaching to hearing adults (and children/youth) has expanded local and broader collective understandings of deaf culture and cross-cultural narrative styles.

Ultimately, after over a century of largely being pushed aside, sign languages are no longer as overlooked and dismissed as they once were and instead have made increasingly frequent appearances in popular culture in North America (e.g., sign language rap battles, sign language in movies and television, deaf celebrities). The increase in photo- and video-based social media platforms (e.g., Facebook, SnapChat, Instagram) have offered visual languages more space for expression and visibility. It could also be said that issues of which ASL and deaf rights are a part (e.g., sign language rights, deaf equity and accessibility) strike a chord in an era of liberal social justice (e.g., \#MeToo, Black Lives Matter). The role that ASL teachers play in shaping hearing discourse around sign languages, deaf people, and social justice was far beyond the scope of this research study, but is worth investigation. 


\section{Parting Words}

Over the course of creating this dissertation and certainly in the decades prior, much has shifted in the ASL landscape - scholarship is growing, teaching practices may be evolving, the social and political climate has becoming more accepting of sign languages, and so on. Likewise, the fields of applied linguistics and applied sign linguistics are continuing to grow as new discoveries and new scholarship shift the knowledge landscape. As I quoted earlier in this dissertation: Nothing stands still (Cleary, 2013; Chapter Four). Cleary uses this expression to point to postcolonial theories of multiply-positioned identities that recognize the dynamic nature of identity. This is a valuable orientation in my dissertation in that it reminds me-and perhaps fellow readers - to approach teachers' stories as ever-changing and therefore ever-impactful. Ivanič (1998) made similar references to this notion when she wrote:

Every time a writer constructs a discoursal self which also draws on less privileged positions for self-hood they are, like a drop in the ocean, infinitesimally redefining the possibilities for self-hood which will, in turn, be available to future writers. (p. 28).

Here, Ivanič's channels a social constructionist point of view and how a writer can "contest[ ] dominant constructions of reality" (p. 13) and potentially provoke something akin to a Kuhnian (1962/2012) revolution, one small piece at a time.

This revolution is already underway with each new teaching and learning experience that shapes the many ASL stakeholders' views of the future of their language and communities. Whatever its direction, I hope at the fore of the revolution is the sentiment that ASL teaching offers a rich opportunity to speak across languages and 
cultures, to unite professionals, to look at teachers as transformative intellectuals - to borrow from critical education theorist Henry Giroux (1988) — as in, as individuals who, through collective efforts, can be a major force for social good. Likewise, as suggested earlier in this dissertation, ASL has significant transformative potential (Norton \& Toohey, 2004) to change hearing people's attitudes that can lead to much-needed social change for deaf people (Turner, 2009).

Completing this dissertation, especially my early collaboration with the SLIC representatives, reinforced the need to nurture local and national communities of ASL teachers to help support its growth and self-definition as a profession. It also served to show how such work could benefit from a more multi-disciplinary approach, where applied linguists, educational researchers, and deaf studies scholars, as well as members from across sign language communities weave their local and disciplinary knowledgessome of them most likely conflicting - to find transformative solutions to complex issues. 


\section{References}

Adam, R. (2015). Dissemination and transfer of knowledge to the Deaf community. In E. Orfanidou, B. Woll, \& G. Morgan (Eds.), Research methods in sign language studies: A practical guide (pp. 41-52). Oxford, UK: John Wiley \& Sons.

American Sign Language Teachers' Association (2020). Getting started. Retrieved from https://aslta.org/certification-begins-here/

Appadurai, A. (1991). Global ethnoscapes: Notes and queries for a transnational anthropology. In R. Fox (Ed.) Recapturing Anthropology (pp. 191-210). Santa Fe, NM: School of American Research Press.

Armstrong, D. F. (1988). Some notes on ASL as a "foreign" language. Sign Language Studies, 59(1), 231-239.

Ashton, G., Cagle, K., Brown Kurz, K., Newell, W., Peterson, R., \& Zinza, J. (2014). Standards for learning American Sign Language. Retrieved from http://www.aslta.org/wp-content/uploads/2014/07/ National_ASL_ Standards.pdf

Ashton, G., Cagle, K., Forestal, L., Greer, L., Jacobowitz, E. L., \& Newell, B. (2020). History of the American Sign Language Teachers' Association (ASLTA). Retrieved from: https://aslta.org/about/history/

Atherton, M., \& Barnes, L. (2012). Deaf people as British Sign Language teachers: Experiences and aspirations. Deafness \& Education International, 14(4), 184198. 
Aubrecht, A., \& Furda, E. (2012). Exploring hearing privilege. Retrieved from http://www.deafecho.com/2012/08/exploring-hearing-privilege/

Baker-Shenk, C. L., \& Cokely, D. (1991). American Sign Language: A teacher's resource text on grammar and culture. Washington, DC: Gallaudet University Press.

Baker, C., \& Cokely, D. R. (1980). American Sign Language: A teacher's resource text on curriculum, methods, and evaluation. Burtonsville, MD: Sign Media.

Bakhtin, M. M. (1981). The dialogic imagination: Four essays by M. M. Bakhtin (M. Holquist, Ed.; C. Emerson \& M. Holquist, Trans.). Austin, TX: University of Texas Press.

Battison, R., \& Carter, S., (1981). The academic status of sign language. In F. Caccamise, M. Garretson, \& U. Bellugi (Eds.), Teaching American Sign Language as a second/foreign language: Proceedings of the third national symposium on sign language research and teaching. Silver Springs, MD: National Association of the Deaf.

Bauman, H. D. L. (2004). Audism: Exploring the metaphysics of oppression. Journal of Deaf Studies and Deaf Education, 9(2), 239-246.

Bauman, H. D. L. (Ed.). (2008). Open your eyes: Deaf studies talking. Minneapolis, MN: University of Minnesota Press.

Bauman, H. D. L., \& Murray, J. J. (Eds.). (2014). Deaf Gain: Raising the stakes for human diversity. Minneapolis, MN: University of Minnesota Press. 
Beal-Alvarez, J. S., \& Scheetz, N. A. (2015). Pre-service teacher and interpreter American Sign Language abilities: Self-evaluations and evaluations of deaf students' narrative renditions. American Annals of the Deaf, 160(3), 316-333.

Beal, J. (2020). University American Sign Language (ASL) second language learners: Receptive and expressive ASL performance. Journal of Interpretation, 28(1), Article 1.

Beal, J. S., Scheetz, N. A., Trussell, J. W., McAllister, A., \& Listman, J. (2018). University American Sign Language learners: Longitudinal self- and faculty evaluation ratings. Journal of Interpretation, 26(1), 1-27.

Belka, R. W. (2000). Is American Sign Language a “foreign” language? NECTFL Review, 48, 45-52.

Bellugi, U., \& Fischer, S. (1972). A comparison of sign language and spoken language. Cognition, 1(2-3), 173-200.

Bill C-81: An Act to ensure a barrier-free Canada. (2019). 42nd Parliament, 1st session. Retrieved from the Parliament of Canada website: https://www.parl.ca/Content/Bills/421/Government/C-81/C-81_4/C-81_4.PDF

Branson, J. \& Miller, D. (1993). Sign language, the deaf and the epistemic violence of mainstreaming. Language and Education, 7(1), 21-41.

Breshears, S. (2004). Professionalization and exclusion in ESL teaching. TESL Canada Journal, (Special Issue 4), 23-39.

Brightman, B. L. C. (2014). The relationship between attitudes and perspectives of American Sign Language university students towards deaf people. 
(Unpublished doctoral dissertation). University of South Florida, Tampa, FL, USA.

Brueggemann, B. J. (2007). Deaf lives leading Deaf lives. Sign Language Studies, 7(2), $111-134$.

Brueggemann, B. J. (2008). The little language that could. In T. Berberi, E. C.

Hamilton, \& I. M. Sutherland (Eds.), Worlds apart?: Disability and foreign language learning (pp. 93-106). New Haven, CT: Yale University Press.

Bruner, J. (1986). Actual Minds/Possible Worlds. Cambridge, MA: Harvard University Press.

Bruner, J. (1987). Life as Narrative. Social Research, 54, 12-32.

Bruner, J. (1991). The narrative construction of reality. Critical Inquiry, 18, 1-21.

Burns, A. (1999). Collaborative action research for English language teachers. New York: Cambridge University Press.

Calderhead, J. (1996). Teachers: Beliefs and knowledge. In D. Berliner, \& R. Calfee (Eds.), Handbook of educational psychology (pp. 709-725). New York: Macmillan Library Reference.

Calton, C. J. (2014). Teaching respect: Language, identity, and ideology in American Sign Language classes in the United States (Unpublished doctoral dissertation). University of Iowa, Iowa City, IA, USA.

Canadian Association of the Deaf. (2015). Statistics on Deaf Canadians. Retrieved from http://cad.ca/issues-positions/statistics-on-deaf-canadians/

Canadian Cultural Society of the Deaf. (2017). Sign Language Institute of Canada. Retrieved from https://slicanada.ca/slic-2/ 
Canadian Cultural Society of the Deaf. (n.d.). ASLICE Application Brochure. Retrieved from

http://deafculturecentre.ca/Public/Default.aspx?I=590\&n=ASLICE+Application + Brochure

Canadian Hearing Society. (2014). Challenges affecting the Deaf and interpreter communities. Retrieved from https://www.chs.ca/challenges-affecting-deaf-andinterpreter-communities\#2

Canadian Hearing Society. (2020). Vision and mission. Retrieved from https://www.chs.ca/vision-and-mission2020

Carbin, C. (1996). Deaf heritage in Canada: A distinctive, diverse and enduring culture. Toronto, ON: McGraw Hill.

Cenoz, J., \& Gorter, D. (2017). Minority languages and sustainable translanguaging: Threat or opportunity? Journal of Multilingual and Multicultural Development, $38,901-912$.

Chapin, P. (1988). American Sign Language and the Liberal Education. Sign Language Studies, 59, 109-113.

Chase, S. E. (2005). Narrative inquiry: Multiple lenses, approaches, voices. In N. K. Lincoln, \& Y. S. Denizin (2005), The Sage handbook of qualitative research (pp. 651-680). Thousand Oaks, CA: Sage.

Cho, J. \& Trent, A. (2006). Validity in qualitative research revisited. Qualitative Research, 6(3), 319-340.

Clandinin, D. J. (Ed.) (2007). Handbook of narrative inquiry: Mapping a methodology. Thousand Oaks, CA: Sage. 
Clandinin, D. J., \& Connelly, F. M. (1996). Teachers' professional knowledge landscapes: Teacher stories, stories of teachers, school stories, stories of schools. Educational Researcher, 25(3), 24-30.

Clandinin, D. J., \& Connelly, F. M. (2000). Experience and story in qualitative research. San Francisco, CA: Jossey-Bass.

Clarke, J. L. (2007). Foreword. In C. J. Heuer, BUG: Deaf identity and internal revolution. Washington, DC: Gallaudet University Press.

Cleary, L. M. (2013). Nothing stands still. In L. M. Cleary, Cross-cultural research with integrity (pp. 1-20). London: Palgrave Macmillan.

Cohen Efron, A. (2014). We. Have. A. Big. Fucking. Problem. Here. Retrieved from http://www.deafeyeseeit.com/2014/10/13/we-have-a-big-fucking-problem-here/

Conle, C. (2000). Narrative inquiry: Research tool and medium for professional development. European Journal of Teacher Education, 23(1), 49-63.

Connell, R. (2009). Good teachers on dangerous ground: Towards a new view of teacher quality and professionalism. Critical Studies in Education, 50(3), 213229.

Connelly, F. M., \& Clandinin, D. J. (1988). Teachers as curriculum planners: Narratives of experience. Toronto, ON: OISE Press.

Connelly, F. M., \& Clandinin, D. J. (1990). Stories of experience and narrative inquiry. Educational researcher, 19(5), 2-14.

Cook, V. (1999). Going beyond the native speaker in language teaching. TESOL Quarterly, 33(2), 185-209. 
Cooper, S. B. (1998). The academic status of sign language programs in institutions of higher education in the United States (Unpublished doctoral dissertation). Gallaudet University, Washington, D. C., USA.

Cooper, S. B., Reisman J. I., \& Watson, D. (2011). Sign language program structure and content in institutions of higher education in the United States, 1994-2004. Sign Language Studies, 11(3), p. 298-328.

Cooper, S. B., Reisman, J. I., \& Watson, D. (2008). The status of sign language instruction in institutions of higher education: 1994-2004. American Annals of the Deaf, 153(1), 78-88.

Corker, M. (1996). Deaf transitions: Images and origins of deaffamilies, deaf communities, and deaf identities. London: Jessica Kingsley Publishers.

Corwin, K., \& Wilcox, S. (1985). The search for the empty cup continues. Sign Language Studies, 48(1), 249-268.

Cox, B. (2010). Hearing privilege. Retrieved from http://www.deafecho.com/2010/12/hearing-privilege/

Craig, C. J. (1999). Parallel stories: A way of contextualizing teacher knowledge. Teaching and Teacher Education, 15, 397-411.

Craig, C. J. (2000). Stories of schools/teacher stories: A two-part invention on the walls theme. Curriculum Inquiry, 30(1), 11-41.

Craig, C. J. (2007). Story constellations: A narrative approach to contextualizing teachers' knowledge of school reform. Teaching and Teacher Education, 23(2), 173-188. 
Creswell, J. W. (1998). Qualitative research and research design: Choosing among five traditions. London: Thousand Oaks.

Creswell, J. W., \& Miller, D. L. (2000). Determining validity in qualitative inquiry. Theory Into Practice, 39(3), p. 124-130.

Cyrus, B., Katz, E., Parson, F. M., \& Brueggemann, B. J. (2005). Deaf women's lives. Washington, DC: Gallaudet University Press.

Dagenais, D, Walsh, N., Armand, F., \& Maraillet, E. (2008). Collaboration and coconstruction of knowledge during language awareness activities in Canadian elementary school. Language Awareness, 17(2), 139-155.

Davie, E. (2019). How the deaf community is preserving Maritime Sign Language. Retrieved from https://www.cbc.ca/news/canada/nova-scotia/preservingmaritime-sign-language-msl-deaf-community-1.5402328

Davies, A. (1991). The native speaker in applied linguistics. Edinburgh: Edinburgh University Press.

Davis, J. E. (2007). A historical linguistic account of sign language among North American Indians. Multilingualism and Sign Languages: From the Great Plains to Australia, 12, 3-38.

Dewey, J. (1916). Democracy and education (4th printing, 1964). New York: Macmillan

Doré, C. (2015). Assessing the needs of ASL teachers in Canada: A mixed methods study (Unpublished Master's thesis). Carleton University, Ottawa, ON, Canada. 
Eckert, R. C. (2010). Toward a theory of Deaf ethnos: Deafnicity $\approx D /$ deaf (Hómaemon• Homóglosson• Homóthreskon). Journal of Deaf Studies and Deaf Education, 15(4), 317-333.

Eckert, R. C., \& Rowley, A. J. (2013). Audism: A theory and practice of audiocentric privilege. Humanity \& Society, 37(2), 101-130.

Edelist, T. (2015). Listen and speak: Power-knowledge-truth and cochlear implants in Toronto. Disability Studies Quarterly, 35(1).

Eichmann, H. (2009). Planning sign languages: Promoting hearing hegemony?

Conceptualizing sign language standardization. Current Issues in Language Planning, 10(3), 293-307.

Elbaz-Luwisch, F. (2005). Teachers' voices: Storytelling and possibility. Greenwich, CT: Information Age.

Elbaz-Luwisch, F. (2007). Studying teachers' lives and experience: Narrative inquiry into K-12 teaching. In D. J. Clandinin (Ed.), Handbook of narrative inquiry: Mapping a methodology (pp. 357-382). Thousand Oaks, CA: Sage.

Elbaz, F. (1981). The teacher's "practical knowledge": Report of a case study. Curriculum Inquiry, 11(1), 43-71.

Ellis, C., \& Berger, L. (2003). Their story/my story/our story: Including the researcher's experience in interview research. In J. Holstein \& J. Gubrium (Eds.), Inside interviewing; New lenses, new concerns (pp. 157-183). Thousand Oaks, CA: Sage.

Ellis, L. (2018). Languaged lives: A new perspective on language teacher identity. Babel, 52(2), 15-24. 
Ferguson, S. (2014). Privilege 101: A quick and dirty guide. Retrieved from http://everydayfeminism.com/2014/09/what-is-privilege/

Fernandes, J. K., \& Shultz Myers, S. (2009). Inclusive deaf studies: Barriers and pathways. Journal of Deaf Studies and Deaf Education, 15(1), 17-29.

Finocchiaro, M., \& Brumfit, C. (1983). The functional-notional approach: From theory to practice. New York: Oxford University Press.

Forsman, L. (2010). On the changing role of English language education: Promoting respect for difference in the language classroom. Intercultural Education, 21(6), $505-518$.

Foster, S. (1987). Doing research in deafness: Some considerations and strategies. In P. C. Higgins \& J. E. Nash (Eds.), Understanding deafness socially (pp. 3-20). Springfield, IL: Charles C Thomas.

Foster, S. (2001). Examining the fit between deafness and disability. Research in Social Science and Disability, 2, 101-23.

Fox, A. L. C. (2010). Teacher certification of American Sign Language faculty at K-12 and higher education institutions. (Unpublished doctoral dissertation). University of Louisville, Louisville, KY, USA.

Fox, J. (2009). Moderating top-down policy impact and supporting EAP curricular renewal: Exploring the potential of diagnostic assessment. Journal of English for Academic Purposes, 8(1), 26-42.

Frishberg. N., (1988). Signers of tales: The case for literary status of an unwritten language. Sign Language Studies, 59 (Special Issue), 149-170. 
Gagné, A., Gordon, S. S. (2011). Growing new roots: Coming together - New immigrant and Canadian teenagers. In C. Rolheiser, M. Evans, \& M. Gambhir (Eds.), Inquiry into practice: Reaching every student through inclusive curriculum (pp. 56-63). Toronto: Ontario Institute for Studies in Education of the University of Toronto (OISE).

Gagné, A. \& Gordon, S. S. (2015). Leadership education for English language learners as transformative pedagogy. Intercultural Education, 26(6), 530-546.

Geertz, C. (1973). Thick description: Toward an interpretive theory of culture. In C. Geertz, The interpretation of cultures (pp. 3-30). New York: Basic Books.

Giroux, H. A. (1988). Teachers as intellectuals: Toward a critical pedagogy of learning. Boston, MA: Greenwood Publishing Group.

Goertzen, D. (2018). Exploring the skills, qualifications and perspectives of American Sign Language teachers in Manitoba (Unpublished Master's thesis). University of Manitoba, Winnipeg, MB, Canada.

Goldberg, D., Looney, D., \& Lusin, N. (2015). Enrollments in Languages Other Than English in United States Institutions of Higher Education, Fall 2013 [pdf]. Retrieved from http://www.mla.org/pdf/2013_enrollment_survey.pdf

Gordon, M. G., \& Harvey, M. (December, 2009). Provincial review pf ASL/Deaf studies and interpreter education in BC. A report for the BC Ministry of Advanced Education and Labour Market Development. Retrieved from https://docplayer.net/6737775-Provincial-review-asl-deaf-studies-andinterpreter-education-in-bc.html 
Gray, L. L. (2018). American Sign Language program administrators on the status of American Sign Language programs in higher education (Unpublished doctoral dissertation). Argosy University, Atlanta, GA, USA.

Groce, N. E. (1985). Everyone here spoke sign language: Hereditary deafness on Martha's Vineyard. Boston, MA: Harvard University Press.

Guba, E. G., \& Lincoln, Y. S. (2004). Competing paradigms in qualitative research: Theories and issues. In S. N. Hesse-Biber \& P. Leavy (Ed.), Approaches to qualitative research: A reader on theory and practice (pp. 1-38). New York: Oxford University Press.

Gubrium, J. F., \& Holstein, J. A. (1997). The new language of qualitative method. Oxford, UK: Oxford University Press.

Gubrium, J. F., \& Holstein, J. A. (1998). Narrative practice and the coherence of personal stories. The Sociological Quarterly, 39(1), 163-187.

Gubrium, J. F., \& Holstein, J. A. (1999). At the border of narrative and ethnography. Journal of Contemporary Ethnography, 28(5), 561-573.

Gubrium, J. F., \& Holstein, J. A. (2008). Narrative ethnography. In S. Hesse-Biber \& P. Leavy (Eds.), Handbook of emergent methods (pp. 241-264), New York: Guilford Press.

Gubrium, J. F., \& Holstein, J. A. (2009). Analyzing narrative reality. Thousand Oaks, CA: Sage.

Hainer-Violand, J. (2013). (Re)defining priorities: Teachers' perspectives on supporting diverse learners within a flexible curriculum in a high-stakes testing 
atmosphere (Unpublished Master's thesis). University of Toronto, Toronto, ON, Canada.

Haller, B. (1992). Paternalism and protest: The presentation of Deaf persons in the New York Times and Washington Post (Unpublished doctoral dissertation). University of Maryland, College Park, MD, USA.

Harding, A. \& MacFayden, S. (December, 2014) ASL BCCAT Transfer Grid \& Descriptive Pathways Project: Final Report for the ASL Articulation Committee. Retrieved from https://www.bccat.ca/Media/NEWBCCAT/pubs/ASLReport.pdf

Hargreaves, A. (2000). Four ages of professionalism and professional learning. Teachers and Teaching, 6(2), 151-182.

Harley, K. (1989). Language teacher training for native signers: A pilot program at the University of New Brunswick. Sign Language Studies, 62(1), 87-92.

Harmer, J. (2007). The practice of English language teaching (4th Ed.). London: Pearson Longman.

Hauser, P. C., O'Hearn, A., McKee, M., Steider, A., \& Thew, D. (2010). Deaf epistemology: Deafhood and deafness. American Annals of the Deaf, 154(5), 486-492.

Hermann-Shores, P. (2017). Enabling pedagogy and andragogy for 21 st-century sign language users and learners. American Annals of the Deaf, 162(4), 360-364. Higgins, P. C. (1980). Outsiders in a hearing world: A sociology of deafness (Vol. 10). Beverley Hills, CA: Sage Publications. 
Hole, R. (2007). Working between languages and cultures: Issues of representation, voice, and authority intensified. Qualitative Inquiry, 13(5), 696-710.

Holliday, A. (1999). Small cultures. Applied Linguistics, 20(2), 237-264.

Hollingsworth, S., Dybdahl, M., \& Minarik, L. T. (1993). By chart and chance and passion: The importance of relational knowing in learning to teach. Curriculum inquiry, 23(1), 5-35.

Holstein, J. A., \& Gubrium, J. F. (1995). The active interview (Vol. 37). New York: Sage.

Humphries, T. (2004). Communicating across cultures (deaf-hearing) and language learning (Unpublished doctoral dissertation). Union Institute and University, Cincinnati, $\mathrm{OH}, \mathrm{USA}$.

Hyvärinen, M. (2016). Narrative and sociology. Narrative Works, 6(1), p. 38-62.

Ingersoll, R. M. \& Collins, G. J. (2018). The status of teaching as a profession. In J. Ballantine, J. Spade, \& J. Stuber (Eds.), Schools and society: A sociological approach to education (p. 199-213). Newbury Park, CA: Pine Forge Press/Sage.

Ivanič, R. (1998). Writing and identity: The discoursal construction of identity in academic writing. Philadelphia, PA: John Benjamins.

Jacobowitz, E. L. (1999, October 7-10). American Sign Language in higher education: Implications for administrators and teacher trainers. Presented at the American Sign Language Teachers Association Professional Development Conference, Rochester, New York. 
Jacobowitz, E. L. (2005). American Sign Language teacher preparation programs in the United States. Sign Language Studies, 6(1), 76-110.

Jacobowitz, E. L. (2007). A look at teaching standards in ASL teacher preparation programs. Sign Language Studies, 8(1), 4-41.

Johnson, R. E., Liddell, S. K., \& Erting, C. J. (1989). Unlocking the curriculum: Principles for achieving access in deaf education. Gallaudet Research Institute (Working Paper 89-3). Washington, DC: Gallaudet University Press.

Kakistocracy. (n.d.). In Cambridge Dictionary (Online). Retrieved from https://dictionary.cambridge.org/dictionary/english/kakistocracy

Kanda, J., \& Fleischer, L. (1988). Who is qualified to teach American Sign Language? Sign Language Studies, 59, 183-194.

Kannapell, B. (1989). An Examination of Deaf College Students' Attitudes towards ASL and English. In C. Lucas (Ed.), The Sociolinguistics of the Deaf Community (pp. 191-210). San Diego, CA: Academic Press.

Kelly, A. B. (2001). How deaf women construct teaching, language and culture, and gender: An ethnographic study of ASL teachers (Unpublished doctoral dissertation). University of Maryland, College Park, MD, USA.

Kemp, M. (1998). Why is learning American Sign Language a challenge? American Annals of the Deaf, 143(3), 255-259.

Klima, E., \& Bellugi, U. (1979). The signs of language. Cambridge, MA: Harvard University Press.

Komesaroff, L. R., \& McLean, M. A. (2006). Being there is not enough: Inclusion is both deaf and hearing. Deafness \& Education International, 8(2), 88-100. 
Kramsch, C. (1993). Context and culture in language teaching. Oxford, UK: Oxford University Press.

Krausnecker, V. (2015). Ideologies and attitudes toward sign languages: An Approximation. Sign Language Studies, 15(4), 411-431.

Kuhn, T. S. (1962/2012). The structure of scientific revolutions: 50th Anniversary Edition. Chicago, IL: University Of Chicago Press.

Kusters, A., De Meulder, M., \& O'Brien, D. (Eds.). (2017). Innovations in deaf studies: The role of deaf scholars. Oxford, UK: Oxford University Press.

Kusters, A., Green, M., Moriarty, E., \& Snoddon, K. (Eds.). (2020). Sign language ideologies in practice (Vol. 12). Berlin: Walter de Gruyter.

Ladd, P. (2003). Understanding deaf culture: In search of deafhood. Bristol, UK: Multilingual Matters.

Ladd, P. (2005). Deafhood: A concept stressing possibilities, not deficits. Scandinavian Journal of Public Health, 33(66), 12-17.

Ladd, P., \& Lane, H. (2013). Deaf ethnicity, deafhood, and their relationship. Sign Language Studies, 13(4), 565-579.

Lampert, M. (1985). How do teachers manage to teach? Perspectives on problems in practice. Harvard Educational Review, 55, 178-184.

Lampert, M. (2012). Improving teaching and teachers: A "generative dance"? Journal of Teacher Education, 63(5), 361-367.

Lane, H. (1992). The mask of benevolence: Disabling the deaf community. New York: Knopf.

Lane, H. (1995). Constructions of deafness. Disability \& Society, 10(2), 171-190. 
Lane, H. (2002). Do deaf people have a disability? Sign Language Studies, 2(4), 356379.

Lane, H. (2005). Ethnicity, ethics, and the deaf-world. Journal of Deaf Studies and Deaf Education, 10(3), 291-310.

Lane, H., Hoffmeister, R., \& Bahan, B. J. (1996). A journey into the deaf-world. San Diego, CA: Dawn Sign Press.

Lang, H. G., Foster, S., Gustina, D., Mowl, G., \& Liu, Y. (1996). Motivational factors in learning American sign language. The Journal of Deaf Studies and Deaf Education, 1(3), 202-212.

Lave, J. (1993). The practice of learning. In S. Chaiklin \& J. Lave (Eds.), Understanding practice: Perspectives on activity and context (pp. 3-32). Cambridge, UK: Cambridge University Press.

Leigh, I. (2009). A lens on deaf identities. Oxford, UK: Oxford University Press. Lentz, E. M., Mikos, K., \& Smith, C. (1988). Signing Naturally, Level 2. Berkeley, CA: Dawn Sign Press.

Lincoln, Y. S., \& Guba, E. G. (1985). Naturalistic inquiry. Newbury Park, CA: Sage. Looney, D., \& Lusin, N. (2018). Enrollments in languages other than English in United States institutions of higher education, Summer 2016 and Fall 2016 [pdf]: Preliminary Report. Retrieved from https://www.mla.org/content/download/83540/2197676/2016-EnrollmentsShort-Report.pdf

Looney, D., \& Lusin, N. (2019). Enrollments in languages other than English in United States institutions of higher education, Summer 2016 and Fall 2016: 
Final Report [PDF]. Retrieved from

https://www.mla.org/content/download/110154/2406932/2016-EnrollmentsFinal-Report.pdf

Lyons, N. (2007). Narrative inquiry: What possible future influence on policy or practice. In J. D. Clandinin (Ed.), Handbook of narrative inquiry: Mapping a methodology (pp. 600-631). Thousand Oaks, CA: Sage.

Malcolm, K., \& Howard, N. (2009). Traveling the path of excellence in interpreter education: The Canadian experience. In J. Napier (Ed.), International perspectives on sign language interpreter education (pp. 248-266).

Washington, DC: Gallaudet University Press.

Marschark, M., Zettler, I., \& Dammeyer, J. (2017). Social dominance orientation, language orientation, and deaf identity. The Journal of Deaf Studies and Deaf Education, 22(3), 269-277.

McDermid, C. (2005). Investigation of Canadian ASL-English interpreter education programs (Unpublished Master's thesis). York University, Toronto, ON, Canada.

McDermid, C. (2009). Two cultures, one programme: Deaf professors as subaltern? Deafness \& Education International, 11(4), 221-249.

McDermid, C. (2010). Culture brokers, advocates, or conduits: Pedagogical considerations for Deaf interpreter education. International Journal of Interpreter Education, 2, 76-101. 
McDermid, C. A. (2014). Adult second language pragmatic enrichment: The case of ASL (Unpublished doctoral dissertation). York University, Toronto, ON, Canada.

McDonald, J. (2011). Becoming an educational developer: A Canadian university perspective (Unpublished doctoral dissertation). University of Toronto, Toronto, ON, Canada.

McDonald, J., \& Stockley, D. (2008). Pathways to the profession of educational development. Hoboken, NJ: Wiley.

McIntosh, P. (1990). Unpacking the knapsack of white privilege. Independent School, 49(2), 31-36.

McKee, D., \& Woodward, J. (2014). Developing Deaf communities through sign language teacher training. In D. McKee \& R. Rosen (Eds.), Teaching and learning signed languages (pp. 35-64). New York: Springer.

McKee, D., Rosen, R. S., \& McKee, R. (Eds.). (2014). Teaching and learning signed languages: International perspectives and practices. New York: Springer.

Mertzani, M. (2015). How far have we gone with Applied Sign Linguistics in deaf education? Pro-Posições, 26(3), 41-58.

Mikos, K., Smith, C., \& Lentz, E. M. (1988). Signing Naturally, Level 3. Berkeley, CA: Dawn Sign Press.

Miller, C. (2001). The adaptation of loan words in Quebec Sign Language: Multiple sources, multiple processes. In D. Brentari (Ed.), Foreign vocabulary in sign languages: A cross-linguistic investigation of word formation (pp. 139-173). Sussex, UK: Psychology Press. 
Miller, K. (2008). American Sign Language: Acceptance at the university level. Language, Culture, and Curriculum, 21, 226-234.

Mitchell, R., Young, T., Bachleda, B., \& Karchmer, M. (2006). How many people use ASL in the United States? Why estimates need updating. Sign Language Studies, 6, 390-401.

Mitchell, S. H. (1971). The haunting influence of Alexander Graham Bell. American Annals of the Deaf, 116(3), 349-356.

Miyamoto, R., \& Mori, S. (2015). Is Kenyan Sign Language a sister language of ASL? Japanese Journal of Sign Language Studies, 24, 17-30.

Moen, T. (2006). Reflections on the narrative research approach. International Journal of Qualitative Methods, 5(4), 56-69.

Mulrooney, K. J. (2009). Extraordinary from the ordinary: Personal experience narratives in American Sign Language. Washington, DC: Gallaudet University Press.

Napier, J., \& Leeson, L. (2015). Sign language in action. In J. Napier \& L. Leeson (Eds.), Sign Language in Action (pp. 50-84). London: Palgrave Macmillan.

Newell, W. J. (1995a). A profile of professionals teaching American Sign Language. Sign Language Studies, 86(1), 19-36.

Newell, W. J. (1995b). American Sign Language teachers: Practices and perceptions. Sign Language Studies, 87, 141-165.

Newell, W. J. (1995c). Competencies important to teaching ASL: Perceptions between groups. Sign Language Studies, 89, 303-330. 
Newell, W. J., \& Cagle, K. M. (1997/2008/2013). Guidelines for hiring ASL teachers: A resources for the program administrator [pdf]. Retrieved from https://aslta.org/wp-content/uploads/2018/07/Hiring-Recommendations.pdf

Norton, B., \& Toohey, K. (Eds). (2004). Critical pedagogies and language learning. New York: Cambridge University Press.

O'Brien, C. A., \& Placier, P. (2015). Deaf culture and competing discourses in a residential school for the deaf: "Can do" versus "can't do". Equity \& Excellence in Education, 48(2), 320-338.

Olsen, G. W. (1988). Acceptance of ASL: An American ground swell. Sign Language Studies, 59, 100.

Olson, M. R. (1995). Conceptualizing narrative authority: Implications for teacher education. Teaching and Teacher Education, 11(2), 119-135.

Opfer, V. D., \& Pedder, D. (2013). Teacher change and changing teachers via professional development. In C. McLaughlin (Ed.), Teachers learning: Professional development and education (pp. 93-117). Cambridge, UK: Cambridge University Press.

Ouellette, M. (2021, forthcoming). Allocation of resources and support: Novice and experienced French as a Second Language (FSL) teachers in Ontario (Unpublished Master's thesis). Carleton University, Ottawa, ON, Canada.

Padden, C. A., \& Humphries, T. (1988). Deaf in America. Cambridge, MA: Harvard University Press.

Paikeday, T. M. (1985). The native speaker is dead! Toronto: Paikeday. 
Parisot, A. M., \& Rinfret, J. (2012). Recognition of langue des signes québécoise in Eastern Canada. Sign Language Studies, 12(4), 583-601.

Parry, B. C. (2008). Inventing Iris: negotiating the unexpected spatialities of intimacy. History of the Human Sciences, 21(4), 34-48.

Peterson, R. W. (1999). The perceptions of deafness and language learning of incoming ASL students (Unpublished doctoral dissertation). University of California, Riverside, CA, USA.

Peyton, J. K. (1998). ASL as a Foreign Language. (Report NLE-98-2520). Washington, DC: Office of Educational Research and Improvement. (ERIC Document Reproduction Service ED437930). Retrieved from https://files.eric.ed.gov/fulltext/ED437930.pdf\#page=43

Pfeiffer, D. L. (2003). The implementation and administration of American Sign Language programs for foreign language credit in public secondary schools (Unpublished doctoral dissertation). George Washington University, Washington, D. C., USA.

Pine, K. T. (2012). A study of the tenure review experiences of Deaf tenured faculty (Unpublished doctoral dissertation). St. John Fisher College, Rochester, NY, USA.

Polkinghorne, D. E. (1988). Narrative knowing and the human sciences. Albany, NY: SUNY Press.

Polkinghorne, D. E. (1995). Narrative configuration in qualitative analysis. In J. A. Hatch \& R. Wisniewski (Eds.), Life history and narrative (pp. 5-24). London: Falmer Press. 
Polkinghorne, D. E. (2016). Validity Issues in Narrative Research. Qualitative Inquiry, 13(4), 471-486.

Pollard, R. Q. (1992). Cross-cultural ethics in the conduct of deafness research. Rehabilitation Psychology, 37(2), 87.

Preston, P. M. (1992). Mother father deaf: Identity on the margins of culture (Unpublished doctoral dissertation). University of California, San Francisco, CA, USA.

Prinzi, L. (2007). Motivation and second language learning: Implications for ASL (Unpublished Master's thesis). Rochester Institute of Technology, Rochester, NY, USA.

Quer, J. \& Steinbach, M. (2019). Handling sign language data: The impact of modality. Frontiers in Psychology, 10, 483. Retrieved from https://www.frontiersin.org/article/10.3389/fpsyg.2019.00483

Quinto-Pozos, D. (2011). Teaching American Sign Language to hearing adult learners. Annual Review of Applied Linguistics, 31, 137-158.

Reagan, T. (2000). But does it "count"? Reflections on "signing" as a foreign language. Northeast Conference Review, 48, 16-26.

Reagan, T. (2011). Ideological barriers to American Sign Language: Unpacking Linguistic resistance. Sign Language Studies, 11(4), 606-636.

Reviere, R. (Ed.) (1996). Needs assessment: A creative and practical guide for social scientists. New York: Routlege.

Ricoeur, P. (1984). Time and narrative (Vol. 1) (K. Mclaughlin \& D. Pellauer, Trans.). Chicago, IL: University of Chicago Press. 
Ricoeur, P. (1985). History as narrative and practice. Philosophy Today, 29(3), 213222.

Ricoeur, P. (1986). Life: A story in search of a narrator. In M. C. Doeser \& J. N. Kray (Eds.), Facts and Values (pp. 132). Dordrecht, The Netherlands: Martin Nijoff Publishers.

Riessman, C. K. (2008). Narrative Methods for the Human Sciences. Thousand Oaks, CA: Sage.

Robinson, O., \& Henner, J. (2018). Authentic voices, authentic encounters: Cripping the university through American Sign Language. Disability Studies Quarterly, 38(4).

Rogers, A. G. (2007). The unsayable, Lacanian psychoanalysis, and the art of narrative interviewing. In D. J. Clandinin (Ed.), Handbook of narrative inquiry: Mapping a methodology (pp. 99-119). Thousand Oaks, CA: Sage.

Rosen, R. S. (2008). American Sign Language as a foreign language in US high schools: State of the art. The Modern Language Journal, 92(1), 10-38.

Rosen, R. S. (2010). American Sign Language curricula: A review. Sign Language Studies, 10(3), 348-381.

Rosen, R. S. (2014). Between-learners' outside-of-classroom uses of American Sign Language as a foreign language. Sign Language Studies, 14(3), 360-381.

Rosen, R. S. (2015). Learning American Sign Language in high school: Motivation, strategies, and achievement. Washington, DC: Gallaudet University Press.

Rosen, R. S. (2017). American Sign Language: Access, benefits, and quality. Society for American Sign Language Publication (SASL), 1(1), 7-34. 
Rosen, R. S. (Ed.). (2019). The Routledge handbook of sign language pedagogy. New York: Routledge.

Roy, C. B. (2011). Discourse in signed languages. Washington, DC: Gallaudet University Press.

Rutherford, S. D. (1988). The culture of American Deaf people. Sign Language Studies, 59, 129-147.

Sacks, O. (1989). Seeing voices: A journey into the world of the deaf. Berkeley, CA: University of California Press.

Saldaña, J. (2013). The coding manual for qualitative researchers. Thousand Oaks, CA: Sage.

Santini, J. (2011). A cross-cultural riff: Privilege and difference. Retrieved from http://www.deafecho.com/2011/01/a-cross-cultural-riff-privilege-anddifference/

Saskatchewan Human Rights Commission (2016, May 12). Access and Equality for Deaf, deaf, and Hard of Hearing People: A Report to Stakeholders. Retrieved from https://saskatchewanhumanrights.ca/wpcontent/uploads/2020/03/20160512_SHRC_DdHoH_Report.pdf

Sato, T. (2013). Exploring literature on professionalism: Reflections from language teachers in Japanese universities. Bulletin of Seikei University, 47(3), 1-15.

Savin-Baden, M., \& Niekerk, L. V. (2007). Narrative inquiry: Theory and practice. Journal of Geography in Higher Education, 31(3), 459-472. 
Scheetz, N. A., \& Martin, D. S. (2008). National study of master teachers in deaf education: Implications for teacher education. American Annals of the Deaf, 153(3), 328-343.

Schön, D. (1983). The reflective practitioner: How professionals think in action. New York: Basic Books.

Schornstein, R. A. (2005). Teaching ASL in the university: One teacher's journey. Sign Language Studies, 5(4), 398-414.

Scott-Hill, M. (2003). Deafness/disability: Problematising notions of identity, culture and structure. In S. Riddell \& N. Watson (Eds.), Disability, culture and identity (pp. 88-104). New York: Routledge.

Seidman, I. (2006). Interviewing as qualitative research: A guide for researchers in education and the social sciences. New York: Teachers College Press.

Simmons, P. K., \& Beatty, J. N. (2015). Audistocracy [Digital image]. Retrieved from https://audismnegatsurdi.com/2015/11/20/open-letter-for-dr-june-rogersdirector-of-provincial-school-branch/

Singleton, J. L., Jones, G., \& Hanumantha, S. (2014). Toward ethical research practice with Deaf participants. Journal of Empirical Research on Human Research Ethics, 9(3), 59-66.

Sinnett, D. R. (1996). An investigation of how foreign language departments at American colleges and universities view American Sign Language (Unpublished doctoral dissertation). Florida International University, University Park, FL, USA. 
Smart, G. (2007). Ethnographic-based discourse analysis: Uses, issues and prospects. In V. K. Bhatia, J. Flowerdew, \& R. H. Jones (Eds.), Advances in Discourse Studies (pp. 56-66). New York: Routledge.

Smith, C. (1988). Signing Naturally: Notes on the development of the ASL curriculum project at Alta Vista College. Sign Language Studies, 59, 171-182.

Smith, C., Lentz, E. M., \& Mikos, K. (1988). Signing Naturally, Level 1. Berkeley, CA: Dawn Sign Press.

Smith, D. H., \& Andrews, J. F. (2015). Deaf and hard of hearing faculty in higher education: Enhancing access, equity, policy, and practice. Disability \& Society, $30(10), 1521-1536$.

Snoddon, K. (Ed.). (2014). Telling Deaf lives: Agents of change. Washington, DC: Gallaudet University Press.

Snoddon, K. \& Wilkinson, E. 2019. Problematizing the legal recognition of sign languages in Canada. Canadian Modern Language Review, 75(2)128-144.

Sofinski, B. A. (2012). Negotiating the American Sign Language (ASL) maze: Examining the status of ASL in Virginia (Unpublished doctoral dissertation). University of Virginia, Charlottesville, VA, USA.

Stokoe, W. C. (1960/2005). Sign language structure: An outline of the visual communication systems of the American deaf. Journal of Deaf Studies and Deaf Education, 10(1), 3-37

Stokoe, W. C. (1978). Sign language versus spoken language. Sign Language Studies, O(18). 69-90. 
Stokoe, W. C., Casterline, D. C., \& Croneberg, C. G. (1976). A dictionary of American Sign Language on linguistic principles. Washington, DC: Linstok Press.

Stone, C., \& West, D. (2012). Translation, representation and the Deaf 'voice'. Qualitative Research, 12(6), 645-665.

Supalla, S. J., Cripps, J. H., \& Byrne, A. P. (2017). Why American sign language gloss must matter. American Annals of the Deaf, 161(5), 540-551.

Sutherland, I. M. (2008). Everybody wins: Teaching deaf and hearing students together. In T. Berberi, E. C. Hamilton, \& I. M. Sutherland (Eds.), Worlds apart?: Disability and foreign language learning (pp. 42-69). New Haven, CT: Yale University Press.

Sutton-Spence, R., \& West, D. (2011). Negotiating the legacy of hearingness. Qualitative Inquiry, 17(5), 422-432.

Swaney, M. G., \& Smith, D. H. (2017). Perceived gaps and the use of supplemental materials in postsecondary American Sign Language curricula. Sign Language Studies, 17(3), 293-321.

Talbert, J. E., \& McLaughlin, M. W. (1994). Teacher professionalism in local school contexts. American Journal of Education, 102(2), 123-153.

Thomas, D. R. (2017). Feedback from research participants: are member checks useful in qualitative research? Qualitative Research in Psychology, 14(1), 23-41.

Thoryk, R. (2010). A call for improvement: The need for research-based materials in American Sign Language education. Sign Language Studies, 11(1), 100-120. 
Trowler, P. R. \& Turner, G. H. (2002). Exploring the hermeneutic foundations of university life: Deaf academics in a hybrid 'community of practice'. Higher Education, 43, 227-256.

Tuccoli, T. (2009). Hearing privileges at Gallaudet? (Unpublished Master's thesis). Gallaudet University, Washington, D. C., USA.

Tucker, J. (1994/1996). The impact of ASL research on the American Deaf Community. In C. Erting (Ed.), The Deaf Way: Perspectives from the International Conference on Deaf Culture (pp. 365-368). Washington, DC: Gallaudet University Press.

Turner, G. H. (2009). Sign language planning: pragmatism, pessimism and principles. Current Issues in Language Planning, 10(3), 243-254.

Valentine, G., \& Skelton, T. (2008). Changing spaces: The role of the internet in shaping deaf geographies. Social \& Cultural Geography, 9(5), 469-485.

Valli, C., \& Lucas, C. (1992). Linguistics of American Sign Language. Washington, DC: Gallaudet University Press.

Veditz, G. W. (1913) Preservation of the Sign Language. [Video] Retrieved from the Library of Congress, https://www.loc.gov/item/mbrs01815816/

Vermeerbergen, M. (2006). Past and current trends in sign language research. Language \& Communication, 26(2), 168-192.

Warner, N., Doull, E., Falvey, D., Martell, R., McDermott, J., Richman, E. (1997, November). The survival of Maritime Sign Language. [Paper presentation]. From the $21^{\text {st }}$ Annual Meeting of the Atlantic Provinces Linguistic Association, 
Mount Saint Vincent University, Halifax, Nova Scotia. Retrieved from https://www.unb.ca/fredericton/arts/_assets/documents/fr/vol21_1997.pdf

Webster-Wright, A. (2009). Reframing professional development through understanding authentic professional learning. Review of educational research, 79(2), 702-739.

Whitty, G. (2000). Teacher professionalism in new times. Journal of In-Service Education, 26(2), 281-295.

Wilbers, S. (1988). Why America needs Deaf culture: Cultural pluralism and the liberal arts tradition. Sign Language Studies, 59, 195-204.

Wilcox, S. (1988). Introduction: Academic acceptance of American Sign Language. Sign Language Studies, 59, 101-108.

Wilcox, S. (1989). Foreign language requirement? Why not American Sign Language? Washington, DC: Office of Educational Research and Improvement. (ERIC Document Reproduction Service ED309651). Retrieved from https://files.eric.ed.gov/fulltext/ED309651.pdf

Wilcox, S. (2019). Universities that accept ASL in fulfillment of foreign language requirements. Retrieved from http://www.unm.edu/ wilcox/UNM/univlist.html

Wilcox, S., \& Occhino, C. (2016). Historical change in signed languages [Oxford Handbooks Online]. DOI: 10.1093/oxfordhb/9780199935345.013.24

Wilcox, S., \& Peyton, J. K. (1999). American Sign Language as a foreign language. The ERIC Review, 6(1), 159-160.

Wilcox, S., \& Wilcox, P. (1991). Learning to see: American Sign Language as a second language. Englewood Cliffs, NJ: Prentice Hall. 
Winston, E. A. (1999). Storytelling and conversation: Discourse in deaf communities (Vol. 5). Washington, DC: Gallaudet University Press.

Wolf, C. (2019). Open letter to the Standing Committee on Canadian Heritage (CHPC) from the Indigenous Sign Language Council [pdf]. Retrieved from https://www.ourcommons.ca/Content/Committee/421/CHPC/Brief/BR1036602 1/br-external/BCHummingbirdSocietyOfTheDeaf-e.pdf

Woll, B., Kyle, J., \& Deuchar, M. (Eds.). (1981). Perspectives on British Sign Language and deafness. London: Croom Helm.

Woodcock, K., Rohan, M. J., \& Campbell, L. (2007). Equitable representation of deaf people in mainstream academia: Why not? Higher Education, 53(3), 359-379.

Woodward, J. C. (1971). Implications for sociolinguistic research among the deaf. Sign Language Studies, 1(1), 1-7.

Yoel, J. (2009). Canada's Maritime Sign Language (Unpublished doctoral dissertation). University of Manitoba, Winnipeg, MB, Canada. 


\section{Appendices}

\section{Appendix A}

Summary of ASL Courses and Programs in Canadian Colleges and Universities (Updated 2019)

Including:

1. List of Canadian Colleges offering ASL

2. List of Canadian Universities offering ASL

1. List of Canadian Colleges offering ASL

\begin{tabular}{|c|c|c|c|c|}
\hline Institution (College) & Department/s / Faculty / School & $\begin{array}{l}\text { Certificate, Diploma, } \\
\text { Other }\end{array}$ & ASL courses & $\begin{array}{l}\text { Deaf } \\
\text { culture } \\
\text { courses }\end{array}$ \\
\hline Camosun College & $\begin{array}{l}\text { School of Health and Human } \\
\text { Services, Continuing Education \& } \\
\text { Contract Training }\end{array}$ & $\begin{array}{l}\text { Certificate - ASL Prep or } \\
\text { ASL Basic }\end{array}$ & $\begin{array}{l}\text { ASL Prep I-IV; } \\
\text { ASL Basic I-IV }\end{array}$ & - \\
\hline College of the Rockies & Continuing Education & ASL Certificate & $\begin{array}{l}\text { ASL } \\
\text { Fundamentals; }\end{array}$ & - \\
\hline
\end{tabular}




\begin{tabular}{|c|c|c|c|c|}
\hline & & & $\begin{array}{l}\text { ASL } \\
\text { Comprehensive }\end{array}$ & \\
\hline Douglas College & $\begin{array}{l}\text { Child, Family and Community } \\
\text { Studies; Sign Language Interpretation }\end{array}$ & Diploma SLI & ASL I-IV & Yes \\
\hline $\begin{array}{l}\text { Vancouver Community } \\
\text { College }\end{array}$ & $\begin{array}{l}\text { ASL and Deaf Studies; Sign } \\
\text { Language Studies }\end{array}$ & Certificate & $\begin{array}{l}\text { Sign 010, 020, } \\
031\end{array}$ & $\begin{array}{l}\text { Yes (Deaf } \\
\text { Culture and } \\
\text { Community) }\end{array}$ \\
\hline $\begin{array}{l}\text { Assiniboine Community } \\
\text { College }\end{array}$ & Continuing Studies & $\begin{array}{l}\text { Certificate of } \\
\text { Achievement }\end{array}$ & $\begin{array}{l}\text { ASL 101-103 } \\
\text { and ASL 201- } \\
203\end{array}$ & - \\
\hline $\begin{array}{l}\text { Red River College of } \\
\text { Applied Arts, Science and } \\
\text { Technology }\end{array}$ & Community Services & $\begin{array}{l}\text { Diploma/BA (General) } \\
\text { Linguistics; Certificate of } \\
\text { ASL/English } \\
\text { Interpretation (with } \\
\text { University of Manitoba) }\end{array}$ & Many & - \\
\hline Algonquin College & $\begin{array}{l}\text { Continuing Education/ Language } \\
\text { Studies }\end{array}$ & Certificate & Eight courses & - \\
\hline Durham College & Continuing Education & Certificate & ASL 1-6 & - \\
\hline
\end{tabular}




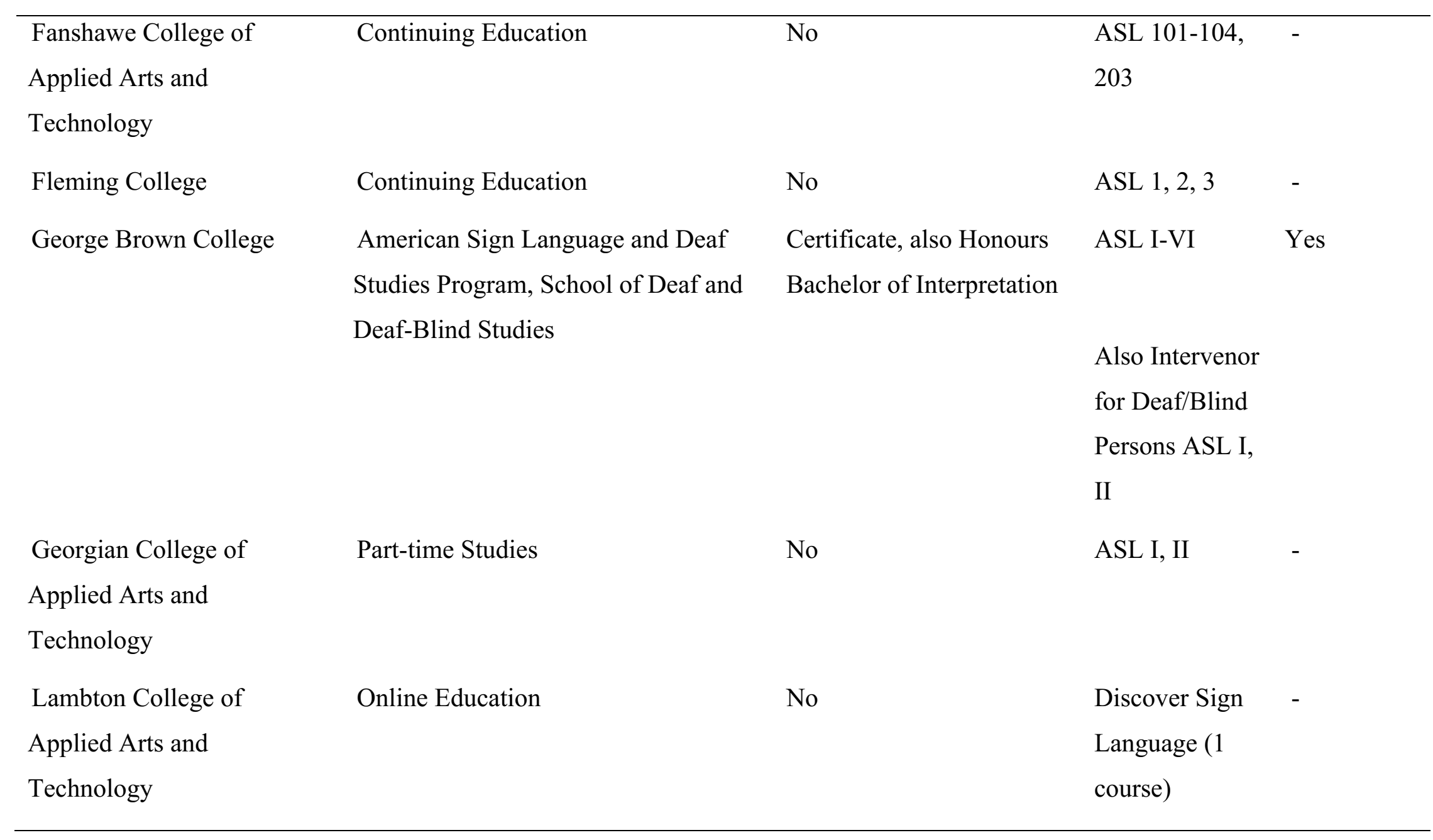




\begin{tabular}{|c|c|c|c|c|}
\hline $\begin{array}{l}\text { Mohawk College of } \\
\text { Applied Arts and } \\
\text { Technology }\end{array}$ & Continuing Education & $\begin{array}{l}\text { Acknowledgement of } \\
\text { Completion }\end{array}$ & ASL 1,2 & - \\
\hline Niagara College & Part-time Studies & No & ASL I-IV & - \\
\hline $\begin{array}{l}\text { St. Clair College of Applied } \\
\text { Arts and Technology }\end{array}$ & Continuing Education & No & ASL 1,2 & - \\
\hline $\begin{array}{l}\text { Seneca College of Applied } \\
\text { Arts and Technology }\end{array}$ & Modern Languages & $\begin{array}{l}\text { Recognition of } \\
\text { Achievement }\end{array}$ & ASL 1-4 & - \\
\hline $\begin{array}{l}\text { Nova Scotia Community } \\
\text { College }\end{array}$ & $\begin{array}{l}\text { Deaf Studies, American Sign } \\
\text { Language-English Interpretation }\end{array}$ & $\begin{array}{l}\text { Deaf Studies-certificate (1 } \\
\text { year) ASLI-Diploma ( } 2 \\
\text { years) }\end{array}$ & $\begin{array}{l}\text { Certificate- } \\
\text { ASL I, II, III; } \\
\text { Diploma- many }\end{array}$ & Yes \\
\hline
\end{tabular}


2. List of Canadian Universities offering ASL

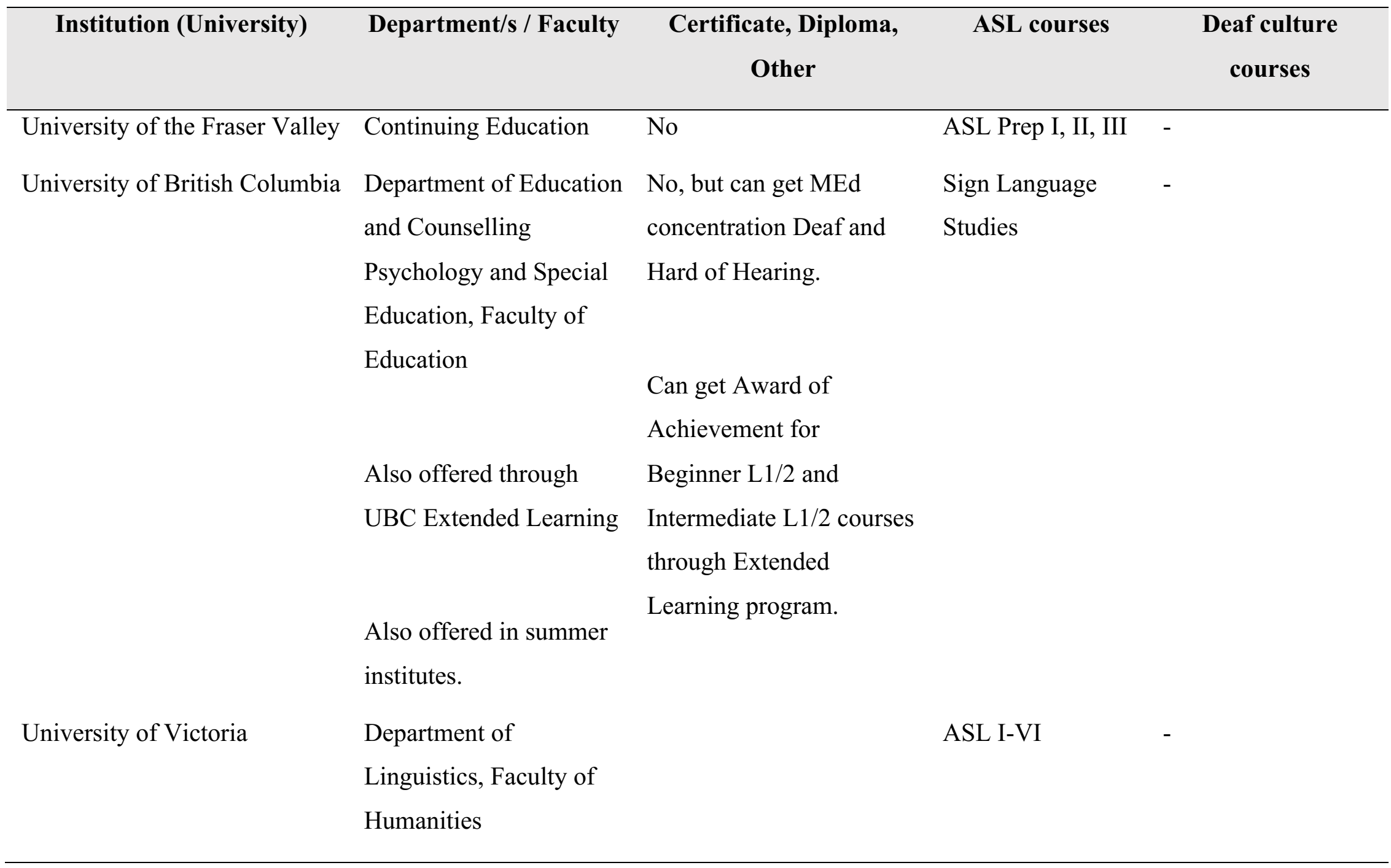




\begin{tabular}{|c|c|c|c|c|}
\hline University of Alberta & $\begin{array}{l}\text { Department of Modern } \\
\text { Languages and Cultural } \\
\text { Studies, Faculty of Arts }\end{array}$ & No & $\begin{array}{l}\text { ASL } 111,112, \\
211,212\end{array}$ & $\begin{array}{l}\text { Associated with } \\
\text { Western Canadian } \\
\text { Centre for Deaf } \\
\text { Studies, but no } \\
\text { courses offered }\end{array}$ \\
\hline University of Calgary & $\begin{array}{l}\text { Division of Linguistics, } \\
\text { School of Languages, } \\
\text { Linguistics, Literature } \\
\text { and Cultures, Faculty of } \\
\text { Arts }\end{array}$ & No & ASL I, II & $\begin{array}{l}\text { Included in ASL } \\
\text { courses }\end{array}$ \\
\hline MacEwan University & $\begin{array}{l}\text { Therapist Assistant, } \\
\text { Speech Language } \\
\text { Pathology Assistant } \\
\text { Program }\end{array}$ & No & THAS216 & - \\
\hline University of Manitoba & $\begin{array}{l}\text { ASL/English } \\
\text { Interpretation Program, } \\
\text { Faculty of Arts }\end{array}$ & $\begin{array}{l}\text { Diploma/BA (General) } \\
\text { Linguistics, plus } \\
\text { Certificate of } \\
\text { ASL/English }\end{array}$ & $\begin{array}{l}\text { ASL 1, 2, } 3 \\
\text { (through Red } \\
\text { River College) }\end{array}$ & $\begin{array}{l}\text { Deaf Culture, Deaf } \\
\text { History (through } \\
\text { Red River College) }\end{array}$ \\
\hline
\end{tabular}




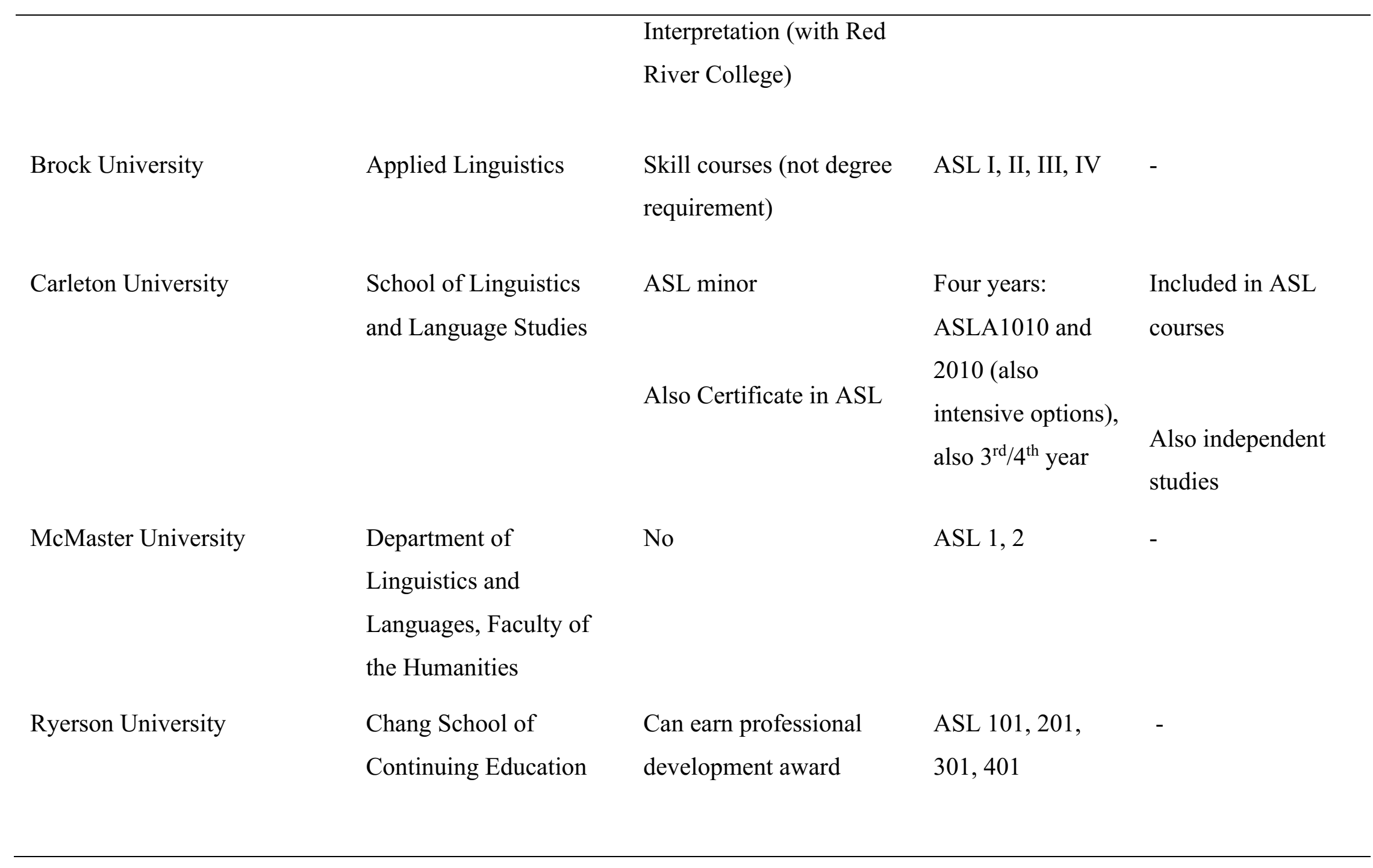




\begin{tabular}{lllll}
\hline Trent University & $\begin{array}{l}\text { Trent Centre for } \\
\text { Language and } \\
\text { Linguistics }\end{array}$ & No & ASL I, II & \\
& Linguistics & No & ASL I & - \\
University of Toronto & No & ASL1 & - \\
University of Waterloo & Applied Language & ASL I, II, II & Included in ASL \\
& Studies & & courses \\
York University & $\begin{array}{l}\text { Department of } \\
\text { Language, Literature, } \\
\text { and Linguistics, Faculty } \\
\end{array}$ & & & \\
& No Liberal Arts and & & \\
\hline
\end{tabular}




\section{Appendix B}

Mock-up Sample Screenshot of Survey from Doré (2015)

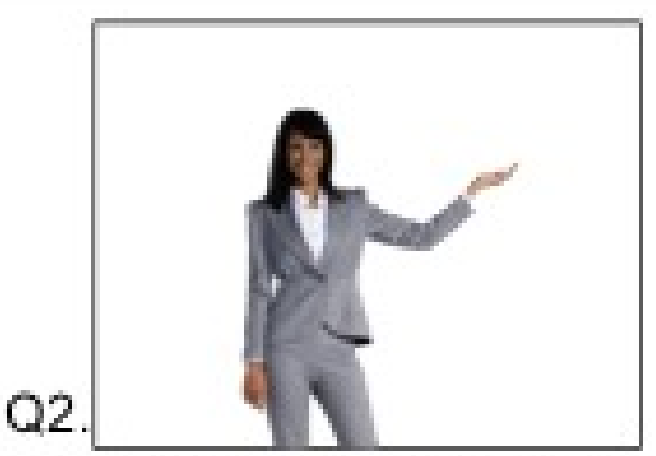

Are you currently or previously within 2 years) a teacher of ASL in a Canad an college, unversity, or communty program?
a Yes
1. $\mathrm{No}$ 


\section{Appendix C \\ Survey from Doré (2015)}

\section{(ASL video)}

See below for English.

\section{Introduction}

This survey is part of a research study by Christina (Nina) Doré for the purposes of better understanding the wants and needs of Canadian ASL teachers. Your participation will increase knowledge of the Canadian ASL teaching community. The study is part of her doctoral program in Applied Linguistics and Discourse Studies at Carleton University under the supervision of Dr. Janna Fox.

\section{Procedures \& anonymity}

The survey will take approximately 15 minutes to complete. Please complete the survey in one sitting. If there is a question you are not comfortable answering feel free to skip it and continue to the next question. There are no known risks associated with your participation in this study. This is an anonymous survey. None of the information you submit can be traced back to you individually and so you will not be able to withdraw from the study after you submit your responses.

\section{Confidentiality}

Your responses will be available only to Nina and her supervisor, Dr. Fox, and may be used in future research about ASL teaching in Canada.

\section{Results}

The results of this study will be published in a report available on the Sign Language Instructors of Canada (SLIC) website (www.deafculturecentre.ca/Public/Default.aspx?I=585\&n=SLIC\%2fASLICE). If you would like a copy of the report contact Nina at christinadore@cmail.carleton.ca. The results will also be published as part of her doctoral dissertation, as an academic journal article, and possibly as a conference paper.

\section{Privacy policy}

Please do not reproduce or borrow any of the contents of this survey. Survey data is collected through FluidSurveys is stored on Canadian servers. The security and privacy policy for FluidSurveys can be found at the following link: https://fluidsurveys.com/canada/data-privacy-canada/

\section{Questions?}

For more information about this study, feel free to contact Nina directly at ------. This study has been reviewed and cleared by the Carleton University Research Ethics Board (CUREB). If you have questions or concerns about your rights as a participant or about the way the study is being conducted you may contact: Professor Andy Adler, Chair, or Professor Louise Heslop, Vice-Chair, Research Ethics Board, Carleton University 
Research Office, Carleton University, 1125 Colonel By Drive, Ottawa, Ontario, K1S 5B6, tel: ------

\section{Consent to participate}

By completing the survey you are indicating you have read and understood the above information and agree to participate in this research study. Click "Next" below to begin the survey.

$* * *$

(ASL video)

See below for English.

\section{Welcome to the survey!}

Before we begin, here are a few quick tips on how to use this survey. Every page has one or more questions, all signed (with captions) and written (below the video). It is important to scroll down the page to see all of the questions. At the bottom of the page are three buttons: "Back" takes you to the previous page, "Next" takes you to the next page, and "Discard" will close the survey and delete all of your answers up to that point.

\section{Video tips}

The videos are captioned - to turn on/off the captions, click the CC button at the bottom of the video. If the video quality is low, click the gear ("Settings") button at the bottom of the video to select a higher video quality. Or, rewind the video to the beginning. NOTE: if you reload the page during the survey you will erase all of your previous answers on that page.

Are you currently or previously (within 2 years) an ASL teacher at a Canadian college, university, or community program?

$\begin{array}{ll}0 & \text { Yes } \\ 0 & \text { No }\end{array}$

\section{Section A. ASL TEACHING ASSOCIATIONS}

The questions in this section relate to your knowledge, use, and opinions of ASL teaching associations. Please scroll down to see all questions on this page.

Q2.

How familiar are you with the Sign Language Instructors of Canada (SLIC)? Tip: click and drag the hand icon along the bottom bar to select option.

$\begin{array}{ll}\circ & \text { I've never heard of it } \\ \circ & \text { I know it by name only }\end{array}$


I'm somewhat familiar with it

I'm very familiar with it, but I'm not a member

I'm a member

Q3.

How familiar are you with the American Sign Language Teachers' Association (ASLTA)?

I've never heard of it

I know it by name only

I'm somewhat familiar with it

I'm very familiar with it, but I'm not a member

I'm a member

Q4.

Are you familiar with (an)other ASL teaching association(s), whether local, provincial, or national?

$\begin{array}{ll}\bigcirc & \text { No } \\ \bigcirc & \text { Yes, please specify... }\end{array}$

Q5.

What type(s) of information do you get from ASL teaching associations (website or print material)? If you don't access any information through ASL teaching associations, please answer "Not applicable".

Teaching tips (e.g., lesson plans, grading guidelines)

Deaf culture information (e.g., history, arts)

Professional development information (e.g., workshops)

Certification information (e.g., time, cost, location)

Current events (e.g., newsletter, updates)

Job opportunities 
Advice for hiring new teachers

Contact information (e.g., board members)

Other, please specify...

Not applicable

Q6.

To what extent do you dis-/agree with the following statements?

$\begin{array}{llll}\text { Strongly } & \text { Disagree } & \text { Agree } & \text { Strongly } \\ \text { disagree } & \text { somewhat } & \text { somewhat } & \text { agree }\end{array}$

1. I am unsure of what an ASL teaching association is for.

2. I know where to find information about ASL teacher qualification requirements for hiring procedures.

3. I am aware of what teaching associations offer their membership.

4. I feel confident I can find updates on available professional development and certification opportunities.

5. I am unaware of where to find news and current events related to ASL teaching.

6. I don't know what ASL teaching associations exist in Canada.

Section B. ASL-RELATED FORMAL TRAINING: PROFESSIONAL DEVELOPMENT AND CERTIFICATION

The questions in this section relate to your participation and opinions about ASL-related formal training, including professional development (e.g., workshop, course, conference) and education/certification (e.g., certificate, diploma, degree). Please scroll down to see all questions on this page. 


\section{Q7.}

How important do you think it is for ASL teachers to have ASL-related formal training in the following areas?

$\begin{array}{llll}\text { Not } & \text { Somewhat } & \text { Quite } & \text { Very } \\ \text { important } & \text { important } & \text { important } & \text { important }\end{array}$
ASL linguistics
Deaf history and
culture
O
O
Course planning and preparation
$\circ \quad 0$
Grading and student
feedback
Classroom
management

○

O

\section{Q8.}

Thinking about any ASL-related formal training you have taken in the past 2 years, how would you rate the quality of the instruction in the following areas? Skip this question if you haven't taken any formal training in the past 2 years.

$$
\text { Poor Fair Good Excellent }
$$

ASL linguistics

Deaf history and culture

Course planning and preparation

Grading and student feedback

Classroom management
O

O

O

O

O

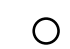

O

O

○

O

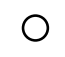

O

O

O

O

Q9.

On average, how often do you participate in the following ASL-related professional development?

Never Rarely Sometimes Often Always

ASL workshop or course

O $\quad 0$

$0 \quad 0$ 
ASL conference (e.g., ASLTA Conference)

Observation and feedback on teaching (you and/or other

ASL teachers)

Individual research (e.g., write articles, read books)

Q10.

How important do you think it is for ASL teachers to participate in ASL-related professional development?

\begin{tabular}{ll}
\hline & Not important \\
$\bigcirc$ & Somewhat important \\
$\bigcirc$ & Quite important \\
\hline & Very important
\end{tabular}

\section{Q11.}

Did you want to participate in more ASL-related professional development than you did in the past 2 years?

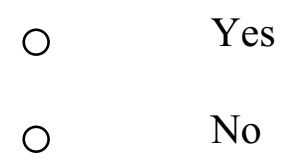

Q12.

If yes, what are some reasons you didn't participate in more?

Nothing suitable was offered

Too expensive

Too far/expensive to travel

Conflict with work schedule

Conflict with family or personal responsibilities seniority) 
$\square \quad$ I found out about it too late

Lack of support/encouragement from department

Other, please specify...

Q13.

Do you have any ASL-related education or certification in ASL teaching, ASL

linguistics, and/or Deaf Studies (e.g., certificate/diploma/degree)?

○ $\quad$ Yes, please specify...

O No

Q14.

How important do you think it is for ASL teachers to have ASL-related education or certification?

O Not important

O Somewhat important

○ Quite important

○ Very important

Q15.

Do you plan on completing any ASL-related certification in the near future? If yes, why?

$\square \quad$ I want to feel more confident in my teaching

I want to connect with other ASL teachers

I want to stay up-to-date with recent trends in ASL teaching

I need certification for a promotion or new job

I'm not sure

Other, please specify...

Q16.

To what extent do you dis-/agree with the following statements?

Strongly Disagree Agree Strongly




$$
\text { disagree somewhat somewhat agree }
$$

1. I do not feel prepared to teach Deaf culture.

2. I am comfortable teaching Deaf history and culture.

3. I enjoy creating new teaching techniques.

4. I am uncomfortable teaching ASL linguistics.

5. I am confident in my ability to evaluate students' ASL skills.

6. I am unsure of how to manage my ASL classroom (e.g., voicing, discipline).

\section{Section C. COLLABORATION WITH OTHER ASL TEACHERS}

The questions in this section relate to your collaboration with other ASL teachers within and outside of your program. Please scroll down to see all questions on this page.

Q17.

On average, how often do you participate in the following activities with other ASL teachers?

Never Rarely Sometimes Often Always

Share teaching materials

(e.g., lesson plans, resources)

$\begin{array}{ll}0 & 0 \\ 0 & 0 \\ 0 & 0\end{array}$

Join classes with another teacher

Observation and feedback on teaching (you and/or another 
teacher)

Team teach (i.e., more than one teacher teaching a class)

Update each other on current events (e.g., training, job opportunities)

Contribute to online ASL

blogs/chat rooms

Recommend research documents related to ASL teaching (e.g., books, journal articles)

Q18.

How important do you think it is for ASL teachers in the SAME program to collaborate with each other?

$\begin{array}{ll}\circ & \text { Not important } \\ \bigcirc & \text { Somewhat important } \\ \bigcirc & \text { Important } \\ \bigcirc & \text { Very important }\end{array}$

Q19.

How important do you think it is for ASL teachers from DIFFERENT programs to collaborate with each other?

O Not important

O Somewhat important

O Important

O Very important

Q20.

What is your supervisor's level of support for collaboration with other ASL teachers?

○ Very unsupportive 


$\begin{array}{ll}\bigcirc & \text { Unsupportive } \\ \bigcirc & \text { Supportive } \\ \bigcirc & \text { Very supportive }\end{array}$

Q21.

To what extent do you dis-/agree with the following statements?

$\begin{array}{llll}\begin{array}{l}\text { Strongly } \\ \text { disagree }\end{array} & \begin{array}{l}\text { Disagree } \\ \text { somewhat }\end{array} & \begin{array}{l}\text { Agree } \\ \text { somewhat }\end{array} & \begin{array}{l}\text { Strongly } \\ \text { agree }\end{array}\end{array}$

1. I would like to feel more connected to ASL teachers in other programs.

2. I feel I am part of an ASL teaching community.

3. I have little contact with my teaching colleagues.

4. I have a strong teacher support network.

5. I wish I knew more ASL teachers.

6. I relate well to other ASL teachers.

\section{DEMOGRAPHIC INFORMATION}

This is the last section of the survey. The questions in this section relate to your demographic information. Please scroll down to see all questions on this page.

Q22.

How many years have you been teaching ASL?

O Less than 1 year

O $\quad 1-2$ years

O $\quad 3-5$ years

O 6-10 years 

11-15 years
O $\quad 15-20$ years
More than 20 years

Q23.

Where do you currently teach ASL? Check all that apply.

$\begin{array}{ll}\square & \text { College/University interpretation program } \\ \square & \text { College program or course } \\ \square & \text { University program or course } \\ \square & \text { Community program or course } \\ \square & \text { Other, please specify } \ldots\end{array}$

\section{Q24.}

What level(s) of ASL do you currently teach? Check all that apply.

1st year/ level 1/ beginner/ introductory/ ASL 101-103

2nd year/ level 2/ high beginner-low intermediate/ ASL 201-203

3rd year/ level 3/ intermediate-advanced/ ASL 301-303

4th year/ level 4/ advanced/ ASL 400

ASL Linguistics

Deaf Studies (e.g., Deaf history and culture)

Other, please specify...

\section{Q25.}

What is your current class size on average?

\begin{tabular}{ll} 
& $1-9$ \\
\hline & $10-19$ \\
\hline & $20-29$
\end{tabular}




$\begin{array}{ll} & 30-39 \\ \text { Q26. } & 40 \text { or more }\end{array}$

How many hours/week are you currently teaching ASL?
Part-time (0-14 hours/week)
Part-time (15-29 hours/week)
O Full-time (30+ hours/week)

\section{Q27.}

Where in Canada do you currently teach ASL?

Western Canada (BC, AB, SK, MB)

Central Canada (ON, QC)

Atlantic Canada (NS, NL, NB, PE)

Northern Canada (YT, NT, NU)

\section{Q28.}

Approximately how many people live in the town/city where you currently teach ASL?

Under 100,000 people

Over 100,000 people

Not sure

Q29.

How many years have you been using ASL?

Less than 1 year

○ 1-2 years

○ $\quad 3-5$ years

6-10 years

O $\quad 11-15$ years 

O
15-20 years
$\bigcirc$
More than 20 years

\section{Q30.}

How involved are you in the Deaf community in Canada?

O Not at all involved

O A little bit involved

○ Quite involved

O Very involved

\section{Q31.}

How old are you?

$\begin{array}{ll} & 25 \text { or under } \\ 0 & 26-40 \\ 0 & 41-55 \\ & \text { over } 55\end{array}$

Q32.

What is your gender?

$\begin{array}{ll}\mathrm{O} & \text { Female } \\ \mathrm{O} & \text { Male } \\ \mathrm{O} & \text { Other } \\ & \text { Prefer not to disclose }\end{array}$

Did we forget to ask something? Please share any additional comments you'd like to make about ASL teaching in Canada. For example, about ASL teaching associations, professional development and certification, or collaboration with other ASL teachers.

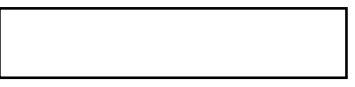

Are you interested in participating in a short follow-up interview? 


$\begin{array}{ll}0 & \text { Yes } \\ 0 & \text { No }\end{array}$

Please provide your current email address and we will message within one week with more information on the follow-up interview. Note: Your email address will be stored separately from your responses. Your email address will not be linked to your responses to identify you in any way.

Email address:
No, thanks.

If you are satisfied with your responses, click "Submit" to submit your survey. You cannot withdraw your survey once submitted. Or click "Delete" and all of your responses will be permanently deleted. Thank you for your participation!
Submit
O Delete 


\section{Appendix D}

\section{Follow-up Interview Guide from Doré (2015)}

\section{ASL teachers' associations}

a. What should a Canadian ASL teachers' association be/not be and do/not do? Why?

b. Is it important to you to have a Canadian ASL teachers' association? Why?

\section{Professional development}

a. Have you done any? If yes, how has it helped/not you in your teaching practice (teaching, planning, grading, etc.)

b. What other PD do you want to see? Look like?

\section{Collaboration other ASL teachers}

a. Has collaboration been productive in your experience?

b. What are some ways you can think of that might encourage more (productive) collaboration between ASL teachers?

Questions? Anything to add?

Thanks! 
Appendix E

\section{CUREB Clearance Certificate \#106640}

Included:

1. Clearance Certificate 2017-2018

2. Clearance Certificate 2018-2019

3. Change to Protocol 


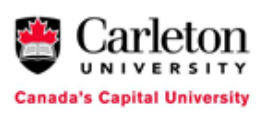

Research Compliance Office 511 Tory | 1125 Colonel By Drive Ottawa, Ontario K1S 5B6 613-520-2600 Ext: 2517 ethics@carleton.ca

\section{CERTIFICATION OF INSTITUTIONAL ETHICS CLEARANCE}

The Carleton University Research Ethics Board-A (CUREB-A) has granted ethics clearance for the research project described below and research may now proceed.

CUREB-A is constituted and operates in compliance with the Tri-Council Policy Statement: Ethical Conduct for Research Involving Humans (TCPS2).

Ethics Protocol Clearance ID: Project \# 106640 Project Team Members: Ms. Christina Doré (Primary Investigator) Mr. Janna Fox (Research Supervisor)

Project Title: Storying the Professionalization of American Sign Language Teaching in Canada [Christina Dore] Funding Source (If applicable):

\section{Effective: April 19,}

2017

Restrictions:

Expires: April 30, 2018.

This certification is subject to the following conditions:

1 Clearance is granted only for the research and purposes described in the application.

2 Any modification to the approved research must be submitted to CUREBA via a Change to Protocol Form. All changes must be cleared prior to the continuance of the research.

3 An Annual Status Report for the renewal of ethics clearance must be submitted and cleared by the renewal date listed above. Failure to submit the Annual Status Report will result in the closure of the file.If funding is associated, funds will be frozen.

4 A closure request must be sent to CUREB-A when the research is complete or terminated.

5 Should any participant suffer adversely from their participation in the project you are required to report the matter to CUREB-A. 


\section{G Carleton \\ Canada's Capital University}

Office of Research Ethics

5110 Human Computer Interaction Bldg | 1125 Colonel By Drive

Ottawa, Ontario K1S 5B6

613-520-2600 Ext: 2517

ethics@,carleton.ca

\section{CERTIFICATION OF INSTITUTIONAL ETHICS CLEARANCE}

The Carleton University Research Ethics Board-A (CUREB-A) at Carleton University has renewed ethics approval for the research project detailed below. CUREB-A is constituted and operates in compliance with the Tri-Council Policy Statement: Ethical Conduct for Research Involving Humans (TCPS2).

Title: Storying the Professionalization of American Sign Language Teaching in Canada [Christina Dore]

Protocol \#: 106640

Project Team Members: Ms. Christina Doré (Primary Investigator)

Janna Fox (Research Supervisor)

Department and Institution: Faculty of Arts and Social Sciences $\backslash$ Linguistics and Language Studies (School of), Carleton University

Funding Source (If applicable):

Effective: April 16, 2018

Expires: April 30, 2019

\section{Restrictions:}

This certification is subject to the following conditions:

1. Clearance is granted only for the research and purposes described in the application.

2. Any modification to the approved research must be submitted to CUREB-A. All changes must be approved prior to the continuance of the research.

3. An Annual Application for the renewal of ethics clearance must be submitted and cleared by the above date. Failure to submit the Annual Status Report will result in the closure of the file. If funding is associated, funds will be frozen. 


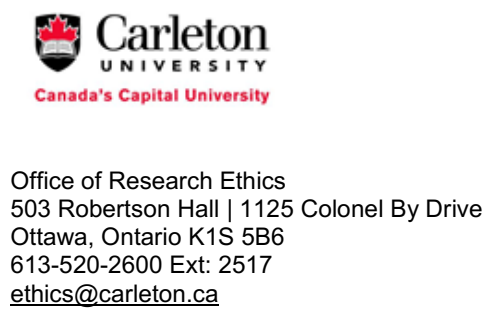

\section{CERTIFICATION OF INSTITUTIONAL ETHICS CLEARANCE}

The Carleton University Research Ethics Board-A (CUREB-A) has granted ethics clearance for changes to protocol to the research project described below and research may now proceed.

CUREB-A is constituted and operates in compliance with the Tri-Council Policy Statement: Ethical Conduct for Research Involving Humans (TCPS2).

Ethics Clearance ID: Project \# 106640

Project Team Members: Ms. Christina Doré (Primary Investigator)

Prof. Janna Fox (Research Supervisor)

Project Title: Storying the Professionalization of American Sign Language Teaching in Canada [Christina Dore]

Funding Source (if applicable):

Effective: December 10, 2018

Expires: April 30, 2019

Upon reasonable request, it is the policy of CUREB, for cleared protocols, to release the name of the PI, the title of the project, and the date of clearance and any renewal(s).

During the course of the study, if you encounter an adverse event, material incidental finding, protocol deviation or other unanticipated problem, you must complete and submit a Report of Adverse Events and Unanticipated Problems Form, found here: https://carleton.ca/researchethics/forms-and-templates/ 


\section{Appendix F}

\section{Invitation Email to Potential ASL Teacher-Participants}

Subject line: Carleton University Study - Storying the Professionalization of American Sign Language Teaching in Canada (Clearance number: 106640)

Dear (name),

My name is Nina (Christina) Doré and I'm currently a PhD student in Applied Linguistics and Discourse Studies (ALDS) at Carleton University, supervised by Dr. Janna Fox.

I am contacting you today with an invitation for you to share your stories about being an ASL teacher in Canada in a study entitled "Storying the Professionalization of American Sign Language Teaching in Canada". The study aims to understand how the profession of ASL teaching has developed in Canada since the 1980s.

Your participation will involve 3-4 interviews (30-60 minutes each), in person or by Skype, between April and September 2017. With your consent, the interviews will be audio/visually recorded and transcribed.

While this project might involve some risks (e.g., if your stories are critical of your personal or professional community), I will take care to protect your identity to the extent that you decide. For example, some or all of your responses can be made anonymous and you will be allowed to request that certain responses not be included in the final project.

You will have the right to end your participation in the study at any time, for any reason, up until two (2) weeks following the final interview. If you choose to withdraw, the information you have provided will be destroyed. All research data, including audiorecordings and any notes will be encrypted. Any hard copies of data (including any 
handwritten notes or USB keys) will be kept in a locked cabinet at Carleton University. Research data will only be accessible to my supervisor and I.

For further information about this study, please contact me (Nina) directly at -------

The ethics protocol for this project was reviewed by the Carleton University Research Ethics Board, which provided clearance to carry out the research. If you have any ethical concerns with the study, please contact Dr. Andy Adler, Chair, Carleton University Research Ethics Board-A (by phone at ------or via email at ------).

Sincerely,

Nina (Christina) Doré 


\section{Appendix G}

\section{Third-Party Recruitment Email for Snowball Sampling (ASL Teachers)}

Subject line: Carleton University Study - Storying the Professionalization of American Sign Language Teaching in Canada

Dear (name),

Nina (Christina) Doré, a student in Applied Linguistics and Discourse Studies (ALDS) at Carleton University, has contacted me asking me to tell you about a study she is doing about ASL teaching to hearing adult learners in Canada. This research is part of her $\mathrm{PhD}$ program in Applied Linguistics and Discourse Studies at Carleton University, supervised by Dr. Janna Fox.

Nina is inviting you to share your stories about being an ASL teacher in Canada by participating in her study. She aims to better understand how the profession of ASL teaching has developed in Canada since the 1980s. Your participation would involve 3-4 interviews (30-60 minutes each), in person or by Skype, between April and September 2017. Nina has explained that your participation is confidential and you can withdraw from the study at any point if you are unwilling to continue. For further information about this study, please contact Nina directly at ------.

The ethics protocol for this project was reviewed by the Carleton University Research Ethics Board, which provided clearance to carry out the research. If you have any ethical concerns with the study, please contact Dr. Andy Adler, Chair, Carleton University Research Ethics Board-A (by phone at ------or via email at ------).

Sincerely, (Sign off) 


\section{Appendix H}

\section{Teacher Interview Question Guide}

\section{Interview 1: Background}

- What was your own ASL learning background - learned as a child, adult, etc.? In class or in a "natural" setting?

- When did you begin teaching ASL? How did you find out about the position? How did you get the position (interview, etc.)?

- What prior experience did you have when hired? Any teaching experience? Other experience?

- What kind of schooling have you completed? Are you planning on completing any more?

- Is teaching ASL part-time or full-time work for you? Do you hope to be doing it more/less in the future?

- What kinds of training and development have you done since beginning formally (courses, workshops, conferences) and informally (mentorship, observation teaching, reading research)?

\section{Interview 2: Experience}

- Are you a member of any Deaf cultural organizations? How does that inform your teaching ASL?

- Are you a member of any professional organizations (interpretation, teaching, etc.)? How does that inform your teaching ASL?

- How much/what kind of input do you get from the wider deaf community re: your teaching? e.g., which signs to use, how to teach, problem students, class management, working with difficult colleagues, etc.

- What kind of curriculum do/have you follow/ed (textbook, etc.)? Why did you choose it, or was it selected for you? Did you receive training on how to use it in the classroom? Does it suit your teaching style and goals? How do you adapt it, if at all? Do you borrow other materials to supplement it? Which ones and why?

- Do you interact with other ASL teachers? Other language teachers? 


\section{Interview 3: Reflections}

- Why have you continued as an ASL teacher? What does teaching ASL to hearing adult learners mean to you? What do you hope is the outcome of your teaching? What do you hope your students do with ASL?

- If ASL for hearing students grows in Canada, what do you think the impact will be - on the Deaf \& hearing communities? On Canadian politics, economy, culture...? 


\section{Appendix I}

\section{ASL Program Administrator Interview Question Guide}

- What is your role in the school/department and in relation to the ASL program? How involved are you with the ASL program's everyday coordination? When/in which aspects are you most/least involved?

- Is the ASL program run in the same way as other programs? What similarities/differences do you observe? How has having an ASL program affected other modern language programs, if at all?

- Are ASL teachers treated/involved in the department in the same way/differently?

- Is the ASL program growing/shrinking? Why, do you think? Why are students choosing/not to take ASL? 
Appendix J

Sample Coding and Inter-Coder Reliability Check

Included:

1. My sample coding (two pages)

2. Coder's sample coding (two pages) 
Nathalie

In terms of first year students, a lot of what I pick up from the deaf community probably isn't too relevant or... cuz it's a very basic level. At that point you don't wanna get too specific about anything

$$
\text { - Ongoing }
$$

Um. ...... the culture has some things about it that first years are probably better off not knowin *laughing. (N: Secret? About like...?) About like... the hard of hearing versus deaf identities an how people relate or refuse to relate... (N: To each other?) Yeah. Yeah... there's a lot of touchy subjects in there. (N: I'm thinking like the identity politics...) Yeah, you can touch a little on deaf vs hearing. you can touch on that. If they ask questions I'll answer them honestly, as best I know... But they don't need to hear all the drama between the deaf and the, oh you're not deaf enough...

- Ongoing

- Connections, she strives for unity. She eschews the dividing topics. She doesn't seem to embody the politics of deaf community. While she understands it, it doesn't seem to have an existential/identity impact of her.

- Here and above, telling a "sticky" story

Yeah, it's been positive yeah. Which is not always what I expected. Maybe I'll learn to be selfconfident yet. Well no, I'm confident in class because I know when I'm teaching anything I'm not, really familiar with, I'll be like, I don't know that, but I'll find out. Um. ...

- Ongoing

- In the interviews she had a way of being confident and shy at once. Maybe nervous? Or in the midst of deciding on herself?

I dunno. I'm becoming more comfortable with my signing again. (N: Again? What was the first time?) When I was in first year, just becoming a student, just joined the deaf community for the first time, I got all confident. Then I started hearing more and more about the, uh, deaf politics things and the animosity between hard of hearing and deaf people. And then I became a teacher and found out about all the politics going on at [University], and I was just like Oooookay, let's be careful. And I'm finally starting to get over that *laughing.

- Past, ongoing, and reflection - pulling from the past, explaining now, and looking ahead

- Eye-opening... learning about politics in the community and in the university; impact on practice

- Story is cautious (for herself and me), not to speak poorly, but still account for tensions

Um... it's fine... Deaf community is like any other community. There are people you're gonna get along with and people you aren't going to get along with. It is what it is. People have their own beliefs and you have to just not get worked up by it.

- Reflective! With previous - builds!

- "The deaf community is like any other community", again she is very humble in a sense she doesn't think of herself (or the deaf community) as anything extraordinary. I think it's a gentle way of commenting on the ups and downs

- She doesn't put the DC on a pedestal. She might have when she was a $1^{\text {st }}$ year student,
Commented [ND1]: Context

Commented [ND2]: Context

Commented [CD3]: Deaf community as resource

Commented [ND4]: Teaching philosophy (macro)

Commented [CD5]: Context; Hearing/HH in de

Commented [ND6]: Teaching philosophy

Commented [ND7]: Learning to teach

Commented [CD8]: Natural teacher; Teaching: confidence..

Commented [ND9]: Context Commented [CD10]: Learning ASL; Deaf politics In progress, ongoing

Commented [ND11]: Constraint! 
going to all of the events and loving it - or maybe not because at that she was just starting to see that this is a place without the same communication and attitudinal barriers that she encountered as a kid/teen.

Generally if it appears consistently, I trust it. (N: Like YouTube? Or, like...) Yeah, like Google and look at the YouTube videos or the online dictionaries or like um... A lot of them are very American, which makes it a little harder. And then with all the LSQ here, too. Sometimes LSQ signs are acceptable here in [City]. But generally if I see the signed used again and again from a few different people. Especially if they're not all from like the same group. So if I see it only

with teachers versus only with one little group in the deaf community I'll be a bit more skeptical that it's not more like a home sign at that point... The more frequently I see it, so if I see it from my interpreters, through the teachers, through the deaf community, and online I'm like yeah. That's good. If I see it only online and through teachers, I'll be like... maybe... but since I'm not $100 \%$ sure I'm not teaching it to my students and they're going to have to gesture! *laughs

- Ongoing, what she's doing now... Also reflections for looking ahead, how she WILL implement it

What kinds of topics did you ask about?) Definitely vocabulary. Every single year I will ask about vocabulary, especially the different countries that students come from. I don't know all the countries. (N: Do you ask about the signs? Or, what vocabulary to include?) I used to ask what vocabulary to include, when I was starting out. I do that less now. But... as the language changes, I find that I'll ask, especially upper year teachers, what versions they teach. Sometimes I ask why as well. I'll generally try to teach my students, OK well, these are all the signs that people use here. Well, maybe not all of them, like if there are a lot of signs, here are some of the signs that people will use. And my preference is this one. But, if you continue doing ASL, other teachers like these.|

- Ongoing, what she's doing now... how past informs now

- Alignment, finding common ground w/ peers

But... The other teachers I was arguing with all supported the shakes, so I'm like OK fine. I'll teach my students the shakes but I'll tell them both are fine because in the deaf community I see both. And in my opinion, if that's what people are using, then both are valid. But because it's in the introduction, I want my students to know what other teachers are using, cuz that's what they're going to be graded on later.

- Ongoing

- Alignment! As above. Looking for where she fits into the bigger picture (university \& community)

Yeah, and then um. In the deaf community I will tell them I teach at [University]. I used to be afraid to do it. Cuz... um. I just started learning sign language ten years ago. You guys have been doing it for 20,30, maybe more years... But most of them seem to think it's great that I'm teaching, and that, I'm skilled. Granted, my fingerspelling when I do it quickly is not pretty. (N: Do you get that feedback?) Oh no. That's just me, judging myself.

- Past to look ahead, forward reflection - how it was \& how she will be moving forward (better signer, she says)

- Some confidence? Confidence-in-development?

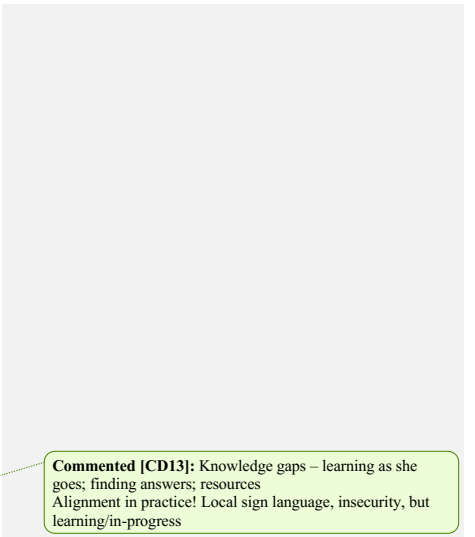
learning/in-progress

Commented [CD14]: Teaching team; alignment; developing

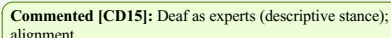
alignment

Commented [ND16]: See lit re: Doré 2015 - experience

[CD17]: Natural teacher; Deaf as expert; insecurity; signing skills 


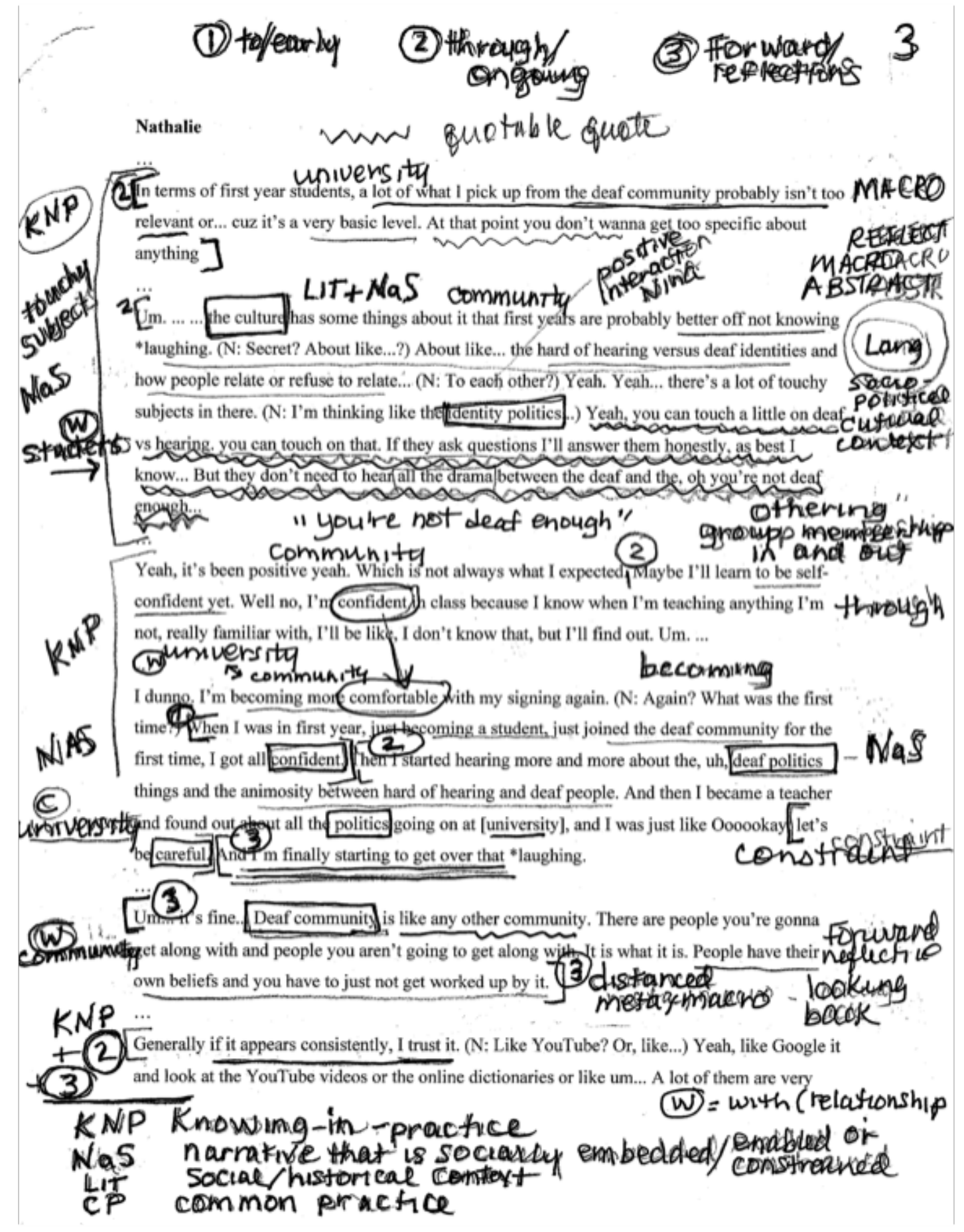




\section{4}

American, which makes it a little harder. And then with all the LSQ here, too. Sometimes LSQ

(2.) signs are acceptable here in [city]. But generally if I see the signed used again and again froma few different people. Especially if they're not all from like the same group. So if I see it only

with teachers versus only with one little group in the deaf community I'll be a bit more skeptical that it's not more like a home sign at that point... The more frequently I see it, so if I see it from

I.

my interpreters, through the teachers, through the deaf community, and online I'm like yeah.

That's good. If I see it only online and through teachers, I'll be like... maybe... but since I'm not

$100 \%$ sure I'm not teaching it to my students and they're going to have to gesture! *laughs

$K N P . .$.
What kinds of topics did you ask about?) Definitely vocabulary. Every single year I will ask

(2) about vocabulary, especially the different countries that students come from. I don't know all the countries. (N: Do you ask about the signs? Or, what vocabulary to include? $\sqrt{\mathrm{I}}$ used to ask what

(1) vocabulary to include, when I was starting out. I do that less now] But... as the lariguage changes,

CP. I find that I'Il ask, especially upper year teachers, what versions they teach. Sometimes I ask why

(2) as well. I'll generally try to teach my students, OK well, these are all the signs that people use here. Well, maybe not all of them, like if there are a lot of signs, here are some of the signs that people will use. And my preference is this one. But, if you continue doing ASL, other teachers like these.

(2) But... The other teacher I was arguing with all supported the shakes, so I'm like OK fine. I'I teach my students the shakes but.J'll tell them both are fine because in the deaf community I see both And in my opinion, if that's what people are using, then both are valid. But because it's in

3 the introduction, I want my students to know what other teachers are using, cuz that's what i) they're going to be graded on later. $\ldots$ KNP. Yeah, and then um. In the deaf community I will tell them I teach at [university. I used to be

CRO $\underbrace{2}$ it for 20,30, maybe more years... But most ge them seem to think it's great that I'm

Min teaching and that, I'm skilled: Granted, my fingerspelling when I do it guickly is not pretty (N: Do you get that feedback?) (D) no. That's just me, judging inysent.

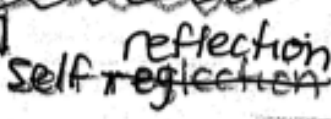




\section{Appendix K}

\section{CEFR Training Description}

\section{ASL/LSQ Advanced Training for Teachers and Instructors}

The Canadian Cultural Society of the Deaf(CCSD) is pleased to announce this successful project titled: Unlocking Culture; Connecting Communities funded in part by the Government of Canada's Social Development Partnerships Program(SDPP) - Disability Component' It is an 18-month project.

This project, spearheaded by CCSD seeks 40 Sign Language instructors to participate in Unlocking Culture: Linking Communities ("Unlocking Culture”). Unlocking Culture aims to increase ASL and LSQ literacy levels and quality of instruction by applying the Common European Framework of Reference for Languages (CEFR) standard to the ASL and LSQ teaching process in Canada.

\section{For ASL and LSQ instructors, Unlocking Culture will include two main steps:}

1. 40 qualified ASL or LSQ instructors will be trained in CEFR techniques in Toronto at the CCSD's DEAF CULTURE CENTRE, Wednesday, February 21 to Sunday, February 25, 2018. Transportation, accommodations and training costs will be covered.

2. 40 ASL and LSQ instructors successfully trained in CEFR will then teach hearing parents of Deaf children or ASL or LSQ interpreters. Your classes will be limited to 10 students per instructor. Classes will either be conducted over our online classroom platform or in person at a location of the instructor's choice. Follow up instructor training will be done "virtually" with one final face-to-face instructors session in the fall 2018 for all instructors together.

\section{DUTIES AND RESPONSIBILITIES:}

- $\quad$ Successfully complete the CEFR training program

- Use the guidelines and techniques of CEFR to develop and teach new courses, curriculum, instructional materials and evaluation tools; review and update course outlines, use CEFR resources for both in person and on-line American Sign Language(ASL) or langue des signes québécoise (LSQ) courses; assist with the 
preparation of course proposals, curriculum updates and the construction of certificate programs

- Apply and maintain standards of quality, operating methods, processes, systems and procedures; implement changes as necessary to maintain a successful ASL or LSQ program; integrate knowledge of industry trends and professional training to continuously improve the project's quality

Participate in ASL or LSQ on and/or off-line course scheduling, post and maintain office hours; participate in the assessment of student learning outcomes; establish, maintain and submit accurate student and instructional records in a timely manner Canadian Cultural Society of the Deaf Collaborate with the Unlocking Culture team, complete reports and class evaluations

\section{REQUIRED QUALIFICATIONS:}

\section{Candidates must have the following:}

- Have experience teaching ASL or LSQ as a second language to adults

- Possess the cultural competence and understanding of Deaf culture, literature and community

- Possess instructional and interpersonal communication skills

- Have the ability to use computer-based training software

- Have sensitivity to successfully interact with diverse populations

- Have ability to design instructional plans for in-class and self-directed learning

- Have knowledge of how to develop objectives for an ASL or LSQ curriculum and instructional objectives of lessons.

\section{Candidates are asked to submit:}

- A 5-min video by December 14, 2017. In this video:

a. discuss your professional experience

b. share your version of the story: "Deaf Timber"

c. state what you think are the 3 top qualities of a good ASL instructor

- Undertake the ASL Proficiency Interview (ASLPI) during the project if you have not had one for the past 3 years. The minimum ASLPI rate expected is $4+$ or better. 


\title{
REPORTING:
}

ASL and LSQ instructors will report to Joanne Cripps, CCSD Executive Director. In addition, instructors will work and collaborate with the project's team members and other stakeholders.

\begin{abstract}
ABOUT CCSD: Founded in 1970, CCSD is dedicated to the advancement of Canada's Deaf communities through promotion and preservation of its' language, culture and heritage. The CCSD works closely with Deaf cultural communities across Canada to improve educational and cultural opportunities of all ages, and has received national and international recognition for its work on improving educational opportunities, and improving access for the Deaf population in Canadian society.
\end{abstract}

Please submit your application marked ASL/LSQ Instructors on the subject line by December 14, 2017 to:

----- -----, Assistant

-- ----- Street

Toronto, ON --- ---

E: 


\section{Appendix L}

\section{Carleton University Petition: "Letter to Carleton University: ASL Instructor"}

To ----- -----, -----, Faculty of Arts and Social Sciences and members of the Carleton University community:

We, the undersigned, are writing to express our profound disappointment that Carleton University has recently hired a nondeaf individual for a full-time, confirmationtrack Instructor I position in American Sign Language (ASL). We note the practice of hiring nondeaf individuals to teach ASL classes has a long history at Carleton and is in fact advertised on the School of Linguistics and Language Studies' web page. As Octavian Robinson and Jon Henner note in their recent Disability Studies Quarterly article, this issue is deeply connected to "larger notions of disability justice and social justice."

Carleton University provides ASL classes for over one thousand students, and numbers of ASL students are greater than numbers of students enrolled in other modern language courses at Carleton. As such, the university financially profits more from ASL classes than from any other modern language. This creates an exploitative relationship between the university and signing deaf communities as the communities of origin of ASL and other signed languages. This relationship is exploitative since there are now more nondeaf than deaf individuals employed in full-time, permanent instructor and program coordinator positions at Carleton. It is also exploitative since the university is silent on the matter of deaf children and their families in Ottawa-Gatineau having few opportunities to learn ASL in early childhood, and no quality ASL-medium education is provided in this region.

Furthermore, the practice of nondeaf instructors teaching nondeaf students ASL as a second language results in poor outcomes for language learning that directly impact the well-being of deaf communities. As a second language in a different modality, ASL requires more years of study than spoken languages, and immersion in the target 
language is not provided when there is limited exposure to deaf communities. Carleton University rejects the use of ASL curricula that have been developed by and with deaf ASL communities and that require training by deaf community organizations, even though development of ASL instructor training by deaf communities has been vital to deaf community development and empowerment. Furthermore, hearing instructors at Carleton are observed to support the use of simultaneous speaking and signing, which is detrimental to ASL acquisition by second-language, second-modality learners and is largely inaccessible to deaf people.

Applicants for ASL instructor positions at Carleton are not required to achieve a minimum score on the American Sign Language Proficiency Interview (ASLPI) as a standardized measure of ASL proficiency. Thus, it is dangerous for Carleton to offer a "certificate" in ASL. Since Carleton has more ASL students than any other Canadian postsecondary institution, Canadian sign language interpreter training programs are faced with an influx of applicants who lack advanced receptive and expressive ASL proficiency as well as cultural competencies. ASL learners who do not develop advanced proficiency become unskilled interpreters who directly impact deaf people's access to employment, healthcare and other public services.

It is not the responsibility of deaf ASL communities to provide instructors for Carleton's ASL classes or to maximize the university's profits. This is especially true since there are no university programs in Canada for deaf students to study ASL teaching, and deaf people face significant barriers to postsecondary education. If no suitable deaf applicants are available, course offerings should be restricted. Limiting the teaching of ASL courses to qualified deaf instructors and streamlining the ASL program at Carleton will render the program more proportionate to the deaf ASL community in OttawaGatineau.

We urge Carleton University to commission an external program review by qualified experts, such as faculty members from Gallaudet University's Department of ASL and Deaf Studies, and engage in consultation with the Sign Language Institute of 
Canada (under the Canadian Cultural Society of the Deaf) regarding program restructuring, curriculum design, assessment, policies, and oversight, including the establishment of a program advisory committee.

Sincerely,

----- -----, -----, Canadian Cultural Society of the Deaf

----- -----, -----, Canadian Cultural Society of the Deaf

----- -----, Sign Language Institute of Canada Advisory Team

----- -----, SignABLE Vi5ion

----- -----, -----, World Association of Sign Language Interpreters

----- -----, -----, Ryerson University

----- -----, ASL Instructor

----- -----, Teacher of the Deaf

----- -----, -----, University of New Mexico 